\title{
ISOMONODROMIC DEFORMATIONS OF CONNECTIONS WITH SINGULARITIES OF PARAHORIC FORMAL TYPE
}

\author{
CHRISTOPHER L. BREMER AND DANIEL S. SAGE
}

\begin{abstract}
In previous work, the authors have developed a geometric theory of fundamental strata to study connections on the projective line with irregular singularities of parahoric formal type. In this paper, the moduli space of connections that contain regular fundamental strata with fixed combinatorics at each singular point is constructed as a smooth Poisson reduction. The authors then explicitly compute the isomonodromy equations as an integrable system. This result generalizes work of Jimbo, Miwa, and Ueno to connections whose singularities have parahoric formal type.
\end{abstract}

\section{INTRODUCTION}

The study of transcendental solutions to differential equations has a long pedigree in mathematics. An important innovation in this field from the turn of the century was Schlesinger's observation that families of solutions to linear differential equations often satisfy interesting nonlinear equations. His work on monodromypreserving deformations of Fuchsian differential equations produced a remarkable family of nonlinear differential equations which satisfy the Painlevé property, namely, that the only movable singularities are simple poles [14.

For example, consider a family of regular singular differential equations on $\mathbb{P}^{1}$ of the form

$$
\frac{d}{d z} \Phi=\sum_{i=1}^{p} \frac{1}{z-x_{i}} A_{i} \Phi
$$

where $x_{1}, \ldots, x_{p} \in \mathbb{P}^{1}$ and $A_{i}$ is a matrix valued holomorphic function in the coordinates $x_{1}, \ldots, x_{p}$. A family of fundamental solutions to (1.1) has constant monodromy if and only if the $A_{i}$ 's satisfy the Schlesinger equations:

$$
d A_{i}=\sum_{j \neq i}\left[A_{i}, A_{j}\right] \frac{d\left(x_{i}-x_{j}\right)}{x_{i}-x_{j}} .
$$

(See [13, IV.1] for a contemporary exposition.) In general, we will refer to the differential equations satisfied by a family of linear differential equations with constant monodromy as isomonodromy equations.

It took almost seventy years for progress to be made on the isomonodromy problem for irregular singular differential equations. In 1981, Jimbo, Miwa, and Ueno characterized the isomonodromy equations for certain generic families of irregular

2010 Mathematics Subject Classification. Primary:14D24; Secondary: 34Mxx, 53D30.

Key words and phrases. meromorphic connections, irregular singularities, moduli spaces, Poisson reduction, fundamental stratum, isomonodromy.

The research of the second author was partially supported by NSF grant DMS-0606300 and NSA grant H98230-09-1-0059. 
singular differential equations 9 . One explanation for the long delay is that the monodromy map for irregular singular point differential equations is significantly more complicated; it involves the asymptotic behavior of solutions along Stokes sectors at each singular point, so it is less explicitly topological than the monodromy map in the regular singular case. The proof of Jimbo, Miwa and Ueno required a much clearer geometric picture of the monodromy map.

In this paper, we will think of linear differential equations in terms of meromorphic connections $\nabla$ on a trivial vector bundle $V$ on $\mathbb{P}^{1}$. After fixing a basis for $V$, we may write $\nabla=d+\alpha$, where $d$ is the usual exterior derivative and $\alpha$ is an $\operatorname{End}(V)$-valued meromorphic one-form. Roughly speaking, if one considers the moduli stack $\mathcal{M}_{\mathrm{DR}}$ of meromorphic connections on $\mathbb{P}^{1}$, there is a formal RiemannHilbert map to the moduli stack $\mathcal{M}_{\mathrm{B}}$ of irregular monodromy representations. 1$]$ An explicit description of the irregular Riemann-Hilbert correspondence may be found in $[10$.

If one can find a smooth family $\mathcal{M}^{\prime}$ that maps to $\mathcal{M}_{\mathrm{DR}}$, the Malgrange-Sibuya theorem [10] implies that the fibers of the monodromy map are a foliation of $\mathcal{M}^{\prime}$. In particular, the isomonodromy equations should correspond to an integrable distribution on $\mathcal{M}^{\prime}$. By an observation of Boalch [1, Appendix], $\mathcal{L} \subset \mathcal{M}^{\prime}$ is a leaf of this foliation if and only if the family of connections corresponding to $\mathcal{L}$ is integrable, i.e., there exists a connection $\bar{\nabla}$ on $\mathcal{L} \times \mathbb{P}^{1}$ with the property that $\left.\bar{\nabla}\right|_{\{x\} \times \mathbb{P}^{1}}$ is a representative of the isomorphism class $x \in \mathcal{M}_{\mathrm{DR}}$. Throughout the paper, we will suppress the monodromy point of view in favor of this integrability condition. As an example, we describe the smooth family of framed connections $\widetilde{\mathcal{M}}_{\text {JMU }}$ constructed by Boalch building on work of Jimbo, Miwa and Ueno.

Fix a finite collection of points $\left(x_{i}\right)_{i \in I} \subset \mathbb{P}^{1}$ and a vector of non-negative integers $\left(r_{i}\right)_{i \in I} 2$ Points in $\widetilde{\mathcal{M}}_{\mathrm{JMU}}$ correspond to isomorphism classes of connections $\nabla=$ $d+\alpha$, singular at $x_{i}$, with the following additional data: there is a collection of framings $g_{i} \in \mathrm{GL}_{n}(\mathbb{C})$ such that the Laurent expansion of $\operatorname{Ad}\left(g_{i}\right) \alpha$ at $x_{i}$ has the form

$$
\operatorname{Ad}\left(g_{i}\right) \alpha=\left(A_{r_{i}} \frac{1}{\left(z-x_{i}\right)^{r_{i}}}+\ldots+A_{1} \frac{1}{z-x_{i}}+A_{0}\right) \frac{d z}{z-x_{i}},
$$

where $A_{i} \in \mathfrak{g l}_{n}(\mathbb{C})$ and the leading term $A_{r_{i}}$ is a regular diagonal matrix. The moduli space $\widetilde{\mathcal{M}}_{\mathrm{JMU}}$ is, in fact, smooth. The isomonodromy equations may be expressed in terms of a Pfaffian system involving terms $\Theta_{i}$, which control the dynamics of the framings, and terms $\Xi_{i}$, which are essentially the principal parts of the curvature of $\bar{\nabla}[9]$.

In 2, the authors describe smooth moduli spaces of framed connections with arbitrary slope, generalizing a construction of 11. The goal of this paper is to study isomonodromic deformations of such connections. The primary technical tool is a local invariant of meromorphic connections called the fundamental stratum, which plays the role of the leading term. A stratum is a triple $(P, r, \beta)$, consisting of a parahoric subgroup $P \subset \mathrm{GL}_{n}(\mathbb{C}[[z]])$, a non-negative integer $r$, and a 'nondegenerate' linear functional on the $r^{t h}$ graded piece associated to the canonical filtration on the Lie algebra of $P$. The relevant condition on connections which

\footnotetext{
${ }^{1}$ Here, DR stands for the "DeRham" theory of meromorphic connections, and B stands for the "Betti" theory of irregular monodromy representations.

${ }^{2}$ There is a slight simplification here: Jimbo, Miwa and Ueno and Boalch allow $x_{i}$ to vary in $\mathbb{P}^{1}$.
} 
assures smoothness of the moduli space is that $\nabla$ must contain a 'regular' stratum. This approach is described in detail in Section 2 .

The primary motivation behind the introduction of fundamental strata into the study of connections comes from the geometric Langlands program, which in this case suggests an analogy between wildly ramified adelic representations of $\mathrm{GL}_{n}$ and irregular monodromy representations of rank $n$ on $\mathbb{P}^{1}$ (see [5]). The fundamental stratum (alternatively, the minimal $K$-type) was originally used as a tool for classifying wildly ramified representations of a reductive group over a $p$-adic field 3, 12. Thus, one hopes that a dictionary between fundamental strata and families of monodromy representations will better illuminate the wild ramification case of the geometric Langlands correspondence.

We conclude with a short overview of the results in this paper. Suppose that $\mathbf{x}=\left(x_{i}\right)_{i \in I} \subset \mathbb{P}^{1}$ is a collection of singular points, $\mathbf{P}=\left(P_{i}\right)_{i \in I}$ is a collection of uniform parahoric subgroups $P_{i} \subset \mathrm{GL}_{n}\left(\mathbb{C}\left[\left[z-x_{i}\right]\right]\right)$, and $\mathbf{r}=\left(r_{i}\right)_{i \in I}$ is a vector of non-negative integers. In Section 3, we give a construction of the moduli space $\widetilde{\mathcal{M}}(\mathbf{x}, \mathbf{P}, \mathbf{r})$ consisting of isomorphism classes of connections with compatible framings on $\mathbb{P}^{1}$ that contain regular strata of the form $\left(P_{i}, r_{i}, \beta_{i}\right)$ at $x_{i}$. By Proposition 3.14 and Theorem 3.15, $\widetilde{\mathcal{M}}(\mathbf{x}, \mathbf{P}, \mathbf{r})$ is a Poisson manifold; moreover, the symplectic leaves correspond to moduli spaces of connections with a fixed formal isomorphism class at each singular point. When all of the parahoric subgroups are maximal, i.e., $P_{i}=\mathrm{GL}_{n}\left(\mathbb{C}\left[\left[z-x_{i}\right]\right]\right), \widetilde{\mathcal{M}}(\mathbf{x}, \mathbf{P}, \mathbf{r})=\widetilde{\mathcal{M}}_{\mathrm{JMU}}$.

In Section 4, we calculate the isomonodromy equations for connections corresponding to points of $\widetilde{\mathcal{M}}(\mathbf{x}, \mathbf{P}, \mathbf{r})$ (Theorem 4.12). In this context, the framing data is given by a coset $U \backslash \mathrm{GL}_{n}(\mathbb{C})$, where $U \subset \mathrm{GL}_{n}(\mathbb{C})$ is the unipotent subgroup determined by $P$. In particular, part of the isomonodromy data is a time dependent flow on an affine bundle over a partial flag manifold. Since $U$ is trivial when $P$ is a maximal parahoric subgroup, this phenomenon is absent in the isomonodromy equations of Jimbo, Miwa and Ueno.

In Section 5, we explicitly show that the isomonodromy equations determine an integrable system on a principal $\mathrm{GL}_{n}(\mathbb{C})$-bundle over $\widetilde{\mathcal{M}}(\mathbf{x}, \mathbf{P}, \mathbf{r})$, corresponding to the moduli space of framed connections with fixed global trivialization. The proof that the corresponding differential ideal is Pfaffian is left to the Appendix. Finally, in Section 6. we compute an explicit example of the isomonodromy equations in the case where $P_{i}$ is an Iwahori subgroup and $r_{i}=1$ for all $i$. To the authors' knowledge, this is a completely new example of an integrable system on a Poisson manifold.

\section{Formal Types}

In this section, we review some results from the geometric theory of fundamental strata and recall how they may be used to associate formal types to irregular singular connections. Let $F=k((z))$ be field of formal Laurent series with coefficients in a field $k$ in characteristic zero, and let $\mathfrak{o} \subset F$ be the corresponding power series ring. Let $\hat{V}$ be an $n$-dimensional $F$ vector space. A lattice chain $\mathcal{L}=\left\{L^{i}\right\}_{i \in \mathbb{Z}}$ is a collection of $\mathfrak{o}$-lattices in $\hat{V}$ satisfying the following properties: $L^{i} \supset L^{i+1}$, and $z L^{i}=L^{i+e}$ with a fixed period $e>0$. We say $\mathcal{L}$ is uniform if $\operatorname{dim}_{k} L^{i} / L^{i+1}=n / e$ for all $i$; the lattice chain is complete if $e=n$. 
Definition 2.1. A uniform parahoric subgroup $P \subset \operatorname{GL}(\hat{V})$ is the stabilizer of a uniform lattice chain $\mathcal{L}$, i.e., $P=\left\{g \in \mathrm{GL}(\hat{V}) \mid g L^{i}=L^{i}\right.$ for all $\left.i\right\}$. The Lie algebra of $P$ is the parahoric subalgebra $\mathfrak{P} \subset \mathfrak{g l}(\hat{V})$ consisting of $\mathfrak{P}=\left\{p \in \mathfrak{g l}(\hat{V}) \mid p L^{i} \subset\right.$ $L^{i}$ for all $\left.i\right\}$. Note that $\mathfrak{P}$ is in fact an associative subalgebra of $\mathfrak{g l}(\hat{V})$. An Iwahori subgroup $I$ is the stabilizer of a complete lattice chain, and an Iwahori subalgebra $\mathfrak{I}$ is the Lie algebra of $I$.

There are natural filtrations on $P$ (resp. $\mathfrak{P}$ ) by congruence subgroups (resp. ideals). For $r \in \mathbb{Z}$, define the $\mathfrak{P}$-module $\mathfrak{P}^{r}$ to consist of $X \in \mathfrak{P}$ such that $X L^{i} \subset$ $L^{i+r}$ for all $i$; it is an ideal of $\mathfrak{P}$ for $r \geq 0$ and a fractional ideal otherwise. The congruence subgroup $P^{r} \subset P$ is then defined by $P^{0}=P$ and $P^{r}=\mathrm{I}_{n}+\mathfrak{P}^{r}$ for $r>0$. Note that these ideals are multiplicative, in the sense that $\mathfrak{P}^{r} \mathfrak{P}^{s}=\mathfrak{P}^{r+s}$. If we fix a form $\nu \in \Omega_{F / k}^{1}$ of order -1 , the pairing

$$
\langle X, Y\rangle_{\nu}=\operatorname{Res} \operatorname{Tr}(X Y \nu)
$$

identifies $\mathfrak{P}^{-r}$ with $\left(\mathfrak{P}^{r+1}\right)^{\perp}$ and $\left(\mathfrak{P}^{r+1} / \mathfrak{P}^{s+1}\right)^{\vee}$ with $\mathfrak{P}^{-s} / \mathfrak{P}^{-r}$. There are similar formulas for $\nu$ of arbitrary order, for example, $\left(\mathfrak{P}^{r+1}\right)^{\perp} \cong \mathfrak{P}^{-r+(1+\operatorname{ord}(\nu) e}$. Throughout this section, we will assume for simplicity that $\operatorname{ord}(\nu)=-1$, but all definitions and results can be stated for other $\nu$.

Definition 2.2. Let $\hat{V}$ be an $F$ vector space, and let $(P, r, \beta)$ be a triple consisting of

- $P \subset \mathrm{GL}(\hat{V})$ a uniform parahoric subgroup;

- $r \in \mathbb{Z}_{>0}$, with $\operatorname{gcd}(r, e)=1$;

- $\beta \in\left(\mathfrak{P}^{r} / \mathfrak{P}^{r+1}\right)^{\vee}$.

After fixing $\nu$ as above, we may identify $\beta$ with a coset $\beta_{\nu}+\mathfrak{P}^{-r+1} \subset \mathfrak{P}^{-r} / \mathfrak{P}^{-r+1}$. Thus, we may choose a representative $\beta_{\nu} \in \mathfrak{P}^{-r}$ for $\beta$. We say that $(P, r, \beta)$ is a uniform stratum if $\beta_{\nu}+\mathfrak{P}^{-r+1}$ contains no nilpotent elements.

In this paper, we are interested in strata that satisfy a 'graded' version of regular semisimplicity. Fix a totally ramified field extension $E / F$ of degree $e$, and let $\mathfrak{o}_{E}$ be the corresponding integral domain. Let

$$
T \cong\left(E^{\times}\right)^{n / e} \subset \operatorname{GL}(\hat{V})
$$

be a maximal torus and let $\mathfrak{t} \cong E^{n / e} \subset \mathfrak{g l}(\hat{V})$ be the corresponding Cartan subalgebra. We denote the identity elements of the Wedderburn components of $\mathfrak{t}$ by $\chi_{j}$, and we write $T(\mathfrak{o})($ resp. $\mathfrak{t}(\mathfrak{o}))$ for $\left(\mathfrak{o}_{E}^{\times}\right)^{n / e} \subset T\left(\right.$ resp. $\left.\mathfrak{o}_{E}^{n / e} \subset \mathfrak{t}\right)$. Moreover, write $\mathfrak{t}^{\mathfrak{b}}$ for the $k$-span of the $\chi_{j}$ and $T^{\mathrm{b}}$ for $\left(\mathfrak{t}^{\mathrm{b}}\right)^{\times}$.

Now, suppose that $(P, r, \beta)$ is a uniform stratum. There is a map $\partial_{\beta, s}: \mathfrak{P}^{s} / \mathfrak{P}^{s+1} \rightarrow$ $\mathfrak{P}^{s-r} / \mathfrak{P}^{s-r+1}$ given by $\partial_{\beta, s}\left(X+\mathfrak{P}^{s}\right)=\operatorname{ad}(X)\left(\beta_{\nu}\right)+\mathfrak{P}^{s-r+1}$.

Definition 2.3. We say that $(P, r, \beta)$ is a regular stratum centralized by $T$ if it satisfies the following conditions:

(1) $T(\mathfrak{o}) \subset P$;

(2) $\operatorname{ker}\left(\partial_{\beta, s}\right)=\mathfrak{t} \cap \mathfrak{P}^{s}+\mathfrak{P}^{s+1}$ for all $s$;

(3) $y_{\beta}=z^{r} \beta_{\nu}^{e}+\mathfrak{P}^{1}$ is a semi-simple element of the algebra $\mathfrak{P} / \mathfrak{P}^{1}$;

(4) when $r=0$ (and hence, $e_{P}=1$ ), the eigenvalues of $y_{\beta}$ are distinct modulo

$\mathbb{Z}$. 
In fact, by [2, Theorem 3.6], $\mathcal{L}$ induces a complete lattice chain on each $\chi_{j}(\hat{V})$ (with period $e$ ). Write $I_{j}$ for the corresponding Iwahori subgroup. It follows from [2, Lemma 2.4] that it is the unique lattice chain $\mathcal{L}_{j}$ with $\mathfrak{o}_{E}^{\times} \subset I_{j}$. Moreover, if we fix a uniformizer $\varpi_{E}$ for $\mathfrak{o}_{E}$, then $\varpi_{E} \mathfrak{I}_{j}=\mathfrak{I}_{j}^{1}$. By [3, Proposition 1.18], we deduce that the matrix $\varpi_{T}=\left(\varpi_{E}, \ldots, \varpi_{E}\right) \in \mathfrak{t}$ satisfies the property $\varpi_{T} \mathfrak{P}=\mathfrak{P} \varpi_{T}=\mathfrak{P}^{1}$.

Recall the following proposition:

Proposition 2.4 ([2, Proposition 2.10]). Let $(P, r, \beta)$ be a regular stratum centralized by $T$, and let $\beta_{\nu} \in \mathfrak{P}^{-r}$ be a representative for $\beta$. There is a morphism of $\mathfrak{t}$-modules $\pi_{\mathfrak{t}}: \mathfrak{g l}(\hat{V}) \rightarrow \mathfrak{t}$ satisfying the following properties:

(1) $\pi_{\mathfrak{t}}$ restricts to the identity on $\mathfrak{t}$;

(2) $\pi_{\mathfrak{t}}\left(\mathfrak{P}^{\ell}\right) \subset \mathfrak{P}^{\ell}$;

(3) the kernel of the induced map

$$
\bar{\pi}_{\mathfrak{t}}:\left(\mathfrak{t}+\mathfrak{P}^{\ell-r}\right) / \mathfrak{P}^{\ell-r+1} \rightarrow \mathfrak{t} /\left(\mathfrak{t} \cap \mathfrak{P}^{\ell-r+1}\right)
$$

is given by the image of $\operatorname{ad}\left(\mathfrak{P}^{\ell}\right)\left(\beta_{\nu}\right)$ modulo $\mathfrak{P}^{\ell-r+1}$;

(4) if $y \in \mathfrak{t}$ and $X \in \mathfrak{g l}(\hat{V})$, then $\langle y, X\rangle_{\nu}=\left\langle y, \pi_{\mathfrak{t}}(X)\right\rangle_{\nu}$;

(5) let $\bar{\pi}_{\mathfrak{t}, \ell}: \mathfrak{P}^{\ell} / \mathfrak{P}^{\ell+1} \rightarrow \mathfrak{t} / \mathfrak{t} \cap \mathfrak{P}^{\ell+1}$ be the induced map, and set $W_{\ell}=\operatorname{ker}\left(\bar{\pi}_{\mathfrak{t}, \ell}\right)$. Then, the induced map $\operatorname{ad}\left(\beta_{\nu}\right): W_{\ell} \rightarrow W_{\ell-r}$ is an isomorphism.

A connection on $\hat{V}$ is a $k$-derivation $\nabla: \hat{V} \rightarrow \hat{V} \otimes \Omega_{F / k}^{1}$. If $\tau$ is a $k$-derivation on $F$, we write $\nabla_{\tau}$ for the composition of $\nabla$ with the inner derivation associated to $\tau: \nabla_{\tau}(v)=i_{\tau}(\nabla(v))$. In particular, let $\tau_{\nu}$ be the derivation with the property that $i_{\tau_{\nu}}(\nu)=1$.

Definition 2.5. Let $(P, r, \beta)$ be a uniform stratum. When $r \geq 1$, we say that $(\hat{V}, \nabla)$ contains $(P, r, \beta)$ if $\nabla_{\tau_{\nu}}\left(L^{i}\right) \subset L^{i-r}$ and $\left(\nabla_{\tau_{\nu}}-\beta_{\nu}\right)\left(L^{i}\right) \subset L^{i-r+1}$ for all $i$. When $r=0$, and thus $e=1$, we say that $(\hat{V}, \nabla)$ contains $(P, 0, \beta)$ if $\left(\nabla_{\tau_{\nu}}-\beta_{\nu}\right)\left(L^{i}\right) \subset L^{i+1}$ for some lattice $L^{i}$.

Given a trivialization $\phi: \hat{V} \stackrel{\sim}{\rightarrow} F^{n}$, we write $[\nabla]_{\phi}$ for the matrix of $\nabla$ with respect to the standard basis of $F^{n}$. By the Leibniz rule, $\nabla=d_{z}+[\nabla]_{\phi}$ where $d_{z}$ is the usual exterior $k$-differential on $F$. The group $\operatorname{GL}_{n}(F)$ acts transitively on the space of trivializations for $F$, and

$$
[\nabla]_{g \phi}=g \cdot[\nabla]_{\phi}:=\operatorname{Ad}(g)[\nabla]_{\phi}-\left(d_{z} g\right) g^{-1} .
$$

If we have fixed a base trivialization, we will shorten $[\nabla]_{g \phi}$ to $[\nabla]_{g}$ and $[\nabla]_{\phi}$ to $[\nabla]$. In general, the left action $g \cdot$ on $\mathfrak{g l}_{n}(F) \otimes \Omega_{F / k}^{1}$ is called a gauge transformation, and we say that two matrices $X, Y \in \mathfrak{g l}_{n}(F) \otimes \Omega_{F / k}^{1}$ are gauge equivalent if there exists $g \in \mathrm{GL}_{n}(F)$ such that $g \cdot X=Y$. Thus, if $\nabla$ and $\nabla^{\prime}$ are connections on $\hat{V}$, and $[\nabla]_{\phi}$ is gauge equivalent to $\left[\nabla^{\prime}\right]_{\phi}$, then $(\hat{V}, \nabla)$ and $\left(\hat{V}, \nabla^{\prime}\right)$ are isomorphic.

Now, suppose that $(P, r, \beta)$ is a regular stratum in $\mathrm{GL}_{n}(F)$ centralized by a torus $T$. We denote the pullback of $P$ and $\beta$ to $\operatorname{GL}(\hat{V})$ by $P^{\phi}$ and $\beta^{\phi}$, respectively.

Theorem 2.6. [2, Theorem 4.13] If $(\hat{V}, \nabla)$ contains the stratum $\left(P^{\phi}, r, \beta^{\phi}\right)$, then there exists $p \in P^{1}$ and a regular element $A_{\nu} \in \mathfrak{t} \cap \mathfrak{P}^{-r}$ such that $p \cdot[\nabla]_{\phi}=A_{\nu} \nu$. Furthermore, the orbit of $A_{\nu} \nu$ under $P^{1}$-gauge transformations contains $\left(A_{\nu}+\mathfrak{P}^{1}\right) \nu$.

Remark 2.7. By [2, Lemma 4.4], the map $\mathfrak{P}^{-r} \rightarrow \mathfrak{P}^{-r} / \mathfrak{P}^{1}$ intertwines the gauge and adjoint actions of $P$. This implies that the functional induced on $\mathfrak{P}^{r} / \mathfrak{P}^{r+1}$ by $A_{\nu}$ coincides with $\beta$. 
We now define the "formal type" of a connection.

Definition 2.8. Let $A \in \mathfrak{P}^{\vee} \backslash\{0\}$, and suppose that $\mathfrak{P}^{r+1}$ is the smallest congruence ideal contained in $A^{\perp}$. Let $\beta$ be the restriction of $A$ to $\mathfrak{P}^{r} / \mathfrak{P}^{r+1}$. We say that $A$ is a formal type if it satisfies the following conditions:

(1) the stratum $(P, r, \beta)$ is regular and centralized by $T$, and

(2) any representative $A_{\nu} \in \mathfrak{P}^{-r}$ for $A$ lies in $\mathfrak{t}+\mathfrak{P}^{1}$.

The connection $(\hat{V}, \nabla)$ has formal type $A$ if there is a trivialization $\phi: \hat{V} \rightarrow$ $F^{n}$ such that $(\hat{V}, \nabla)$ contains the stratum $\left(P^{\phi}, r, \beta^{\phi}\right)$, and $[\nabla]_{\phi}$ is formally gauge equivalent to an element of $\left(A_{\nu}+\mathfrak{P}^{1}\right) \nu$ by an element of $P^{1}$. Finally, we say that two connections that contain regular strata are combinatorially equivalent if the strata have the same parahoric subgroup and slope.

By [2, Corollary 4.16], any two connections with formal type $A$ are formally isomorphic, and the formal type is independent of $\nu$. The converse is false, since any conjugate of $A$ by the relative Weyl group of $T$ is also a formal type for $(\hat{V}, \nabla)$. However, fixing a stratum uniquely determines the formal type. For the rest of the section, we assume (without loss of generality) that $P \subset \mathrm{GL}_{n}(\mathfrak{o})$.

Proposition 2.9. If $\nabla$ contains a regular stratum $\left(P^{\phi}, r, \beta^{\phi}\right)$, there exists a unique formal type $A$ such that $\left.A\right|_{\mathfrak{P}^{r}}=\beta$ and $[\nabla]_{\phi}$ is gauge equivalent to an element of $\left(A_{\nu}+\mathfrak{P}^{1}\right) \nu$ by an element of $\mathrm{GL}_{n}(\mathfrak{o})$.

Proof. By Theorem 2.6 and Remark 2.7 $(\hat{V}, \nabla)$ has a formal type $A$ such that $\left.A\right|_{\mathfrak{P}^{r}}=\beta$, and there exists $p \in P^{1}$ such that $p \cdot[\nabla]_{\phi}=A_{\nu} \nu$, where $A_{\nu} \in \mathfrak{t} \cap \mathfrak{P}^{-r}$. This formal type is unique, since [2, Lemma 3.16] implies that if $[\nabla]_{\phi}$ is gauge equivalent to $A_{\nu}^{\prime} \in \mathfrak{t} \cap \mathfrak{P}^{-r}$ and $\left.A_{\nu}^{\prime}\right|_{\mathfrak{P}^{r}}=\beta$, then $A_{\nu}^{\prime}+\mathfrak{P}^{1}=A_{\nu}+\mathfrak{P}^{1}$.

Finally, throughout the paper we will need to consider a slight variation on the formal type. Choose a uniformizer $\varpi_{E}$ for $E$ such that $\varpi_{E}^{e}=z$; under a suitable embedding $E \hookrightarrow \mathfrak{g l}_{n / e}(\mathbb{C})$, such a way that

$$
\varpi_{E}=\left(\begin{array}{cccc}
0 & 1 & \cdots & 0 \\
\vdots & \ddots & \ddots & \vdots \\
0 & \ddots & 0 & 1 \\
z & 0 & \cdots & 0
\end{array}\right) .
$$

We choose a basis for each $\chi_{j} \hat{V}$ (and hence for $V$ ) such that $\varpi_{T}$ is block diagonal with these blocks. Using this basis, we define $H_{T}=\left(H_{E}, \ldots, H_{E}\right) \in \mathfrak{P}$ as the block diagonal matrix with blocks given by the diagonal matrix $H_{E}=$ $\operatorname{diag}\left(\frac{e-1}{2 e}, \frac{e-3}{2 e}, \ldots, \frac{1-e}{2 e}\right)$. Let $H_{T}^{\prime} \in \mathfrak{P}^{\vee}$ to be the corresponding functional $H_{T}^{\prime}(X)=$ $\left.\operatorname{Tr}\left(H_{T} X\right)\right|_{z=0}$.

Definition 2.10. Let $(\hat{V}, \nabla)$ have formal type $A$. We define the normalized formal type of $(\hat{V}, \nabla)$ to be $\tilde{A}=A+H_{T}^{\prime}$.

Note that if $e=1$, then $\tilde{A}=A$.

Proposition 2.11. Suppose that $(\hat{V}, \nabla)$ contains a regular stratum and has normalized formal type $\tilde{A}$. If $\tilde{A}_{\nu} \in \mathfrak{P}^{-r}$ is a representative for $\tilde{A}$, then there exists $\hat{p} \in P^{1}$ such that $\hat{p} \cdot[\nabla]_{\phi}=\tilde{A}_{\nu} \nu$. 
Proof. If $r=0$ (so $e=1$ ), this is just Theorem [2.6. so assume that $r \geq 1$. Note that $H_{T}^{\prime} \in\left(\mathfrak{t}^{\mathfrak{b}}\right)^{\perp}$. Therefore, part 4 of Proposition 2.4 imply that $\pi_{\mathfrak{t}}\left(H_{T}\right) \in$ $\mathfrak{P}^{1}$. Part 3 of the same proposition shows that there exists $X \in \mathfrak{P}^{r}$ such that $\operatorname{ad}(X) \tilde{A}_{\nu} \in H_{T} \frac{1}{\operatorname{Res}(\nu)}+\mathfrak{P}^{1}$, so there exists $p \in P^{1}$ such that $p \cdot[\nabla]_{\phi}=A_{\nu} \nu$ by Theorem 2.6. Moreover, $\exp (-X) \cdot \tilde{A}_{\nu} \nu \in\left(A_{\nu}+\mathfrak{P}^{1}\right) \nu$, so there exists $p^{\prime} \in P^{1}$ such that $p^{\prime} \exp (-X) \cdot \tilde{A}_{\nu} \nu=A_{\nu} \nu$. It follows that $\left(\exp (X)\left(p^{\prime}\right)^{-1} p\right) \cdot[\nabla]_{\phi}=\tilde{A}_{\nu} \nu$.

Proposition 2.12. Any normalized formal type $\tilde{A}$ is stabilized by $T \cap P^{1}$.

Proof. Since $\operatorname{ad}\left(\mathfrak{P}^{1}\right) H_{T} \in \mathfrak{P}^{1}, P^{1}$ stabilizes $H_{T}+\mathfrak{P}^{1}$. Moreover, the corresponding formal type $A$ is stabilized by $T$.

\section{Moduli SPACes of CONNECTiOns}

In this section, we will describe the moduli space of connections on $\mathbb{P}^{1}(\mathbb{C})$ with compatible framings and fixed combinatorics at each singular point.

First, we recall the construction of the moduli space of framed connections on $\mathbb{P}^{1}(\mathbb{C})$ with fixed formal type at each singular point $[2$. Throughout, $I$ will be a finite indexing set and $\mathbf{x}=\left\{x_{i}\right\}_{i \in I}$ a collection of distinct points in $\mathbb{P}^{1}$. We denote the completion of the function field of $\mathbb{P}^{1}$ at $x_{i}$ by $F_{i}$ and the corresponding power series ring by $\mathfrak{o}_{i}$.

Let $V$ be a trivial rank $n$ vector bundle on $\mathbb{P}^{1}$, i.e., we have fixed a trivialization $V \cong \mathcal{O}_{\mathbb{P} 1}^{n}$. Accordingly, we may identify the space of all global trivializations of $V$ with $\mathrm{GL}_{n}(\mathbb{C})$. Note that a global trivialization determines a local trivialization of $V_{i}:=V \otimes_{\mathcal{O}_{p 1}} F_{i}$, so there is a natural inclusion $\mathrm{GL}_{n}(\mathbb{C}) \hookrightarrow \mathrm{GL}_{n}\left(F_{i}\right)$; moreover, the global sections of $V$ generate a distinguished lattice $L_{i} \subset V_{i}$. Suppose that $\nabla$ is a connection on $V$ with the property that the induced connection on $V_{i}$ has formal type $A_{i}$. We will assume without loss of generality that the associated torus $T_{i}$ is contained in $\mathrm{GL}_{n}\left(\mathfrak{o}_{i}\right)$. Therefore, by [2, Proposition 4.14], $A_{i}$ determines a unique stratum $\left(P_{i}, r, \beta_{i}\right)$ in $\mathrm{GL}_{n}\left(F_{i}\right)$. We also set $\varpi_{i}=\varpi_{T_{i}}$.

Throughout this section, $U_{i}$ will denote the unipotent subgroup $P_{i}^{1} \cap \mathrm{GL}_{n}(\mathbb{C})$ with Lie algebra $\mathfrak{u}_{i}=\mathfrak{P}_{i}^{1} \cap \mathfrak{g l}_{n}(\mathbb{C})$. For simplicity, we write $\mathfrak{g}_{i}$ for the parahoric subalgebra $\mathfrak{g l}_{n}\left(\mathfrak{o}_{i}\right)$; its radical is $\mathfrak{g}_{i}^{1}=t \mathfrak{g}_{i}$. Note that $U_{i} \cong P_{i}^{1} /\left(1+\mathfrak{g}_{i}^{1}\right)$ and $\mathfrak{u}_{i} \cong \mathfrak{P}_{i}^{1} / \mathfrak{g}^{1}$.

Definition 3.1. A compatible framing for $\nabla$ at $x_{i}$ is a global trivialization $g \in$ $\mathrm{GL}_{n}(\mathbb{C})$ with the property that $\nabla$ contains the $\operatorname{GL}\left(V_{i}\right)$-stratum $\left(P_{i}^{g}, r, \beta_{i}^{g}\right)$ defined above. We say that $\nabla$ is framed at $x_{i}$ if there exists such a $g$.

We now define the moduli space of connections with fixed formal type and a specified framing at each singular point. Set $\bar{A}_{i}=\left.A_{i}\right|_{\mathfrak{F}^{1}}$.

Definition 3.2. Let $\widetilde{\mathcal{M}}(\mathbf{A})$ be the the moduli space of isomorphism classes of triples $(V, \nabla, \mathbf{g})$, where

- $\nabla$ is a meromorphic connection on the trivial bundle $V$ with singularities at $\left\{x_{i}\right\}_{i \in I}$;

- $\mathbf{g}=\left\{U_{i} g_{i}\right\}_{i \in I}$, with $g_{i}$ a compatible framing for $\nabla$ at $x_{i}$;

- the formal type $A_{i}^{\prime}$ of $\nabla$ at $x_{i}$ satisfies $\bar{A}_{i}^{\prime}=\bar{A}_{i}$.

The moduli space $\widetilde{\mathcal{M}}(\mathbf{A})$ is built out of "extended" coadjoint orbits $\widetilde{\mathcal{M}}\left(A_{i}\right)$ determined locally by each formal type $A_{i}$. In the following, let $A$ be a formal type. We define $\pi_{\mathfrak{P}}: \mathfrak{g}^{\vee} \rightarrow \mathfrak{P}^{\vee}$ and $\pi_{\mathfrak{P}^{1}}: \mathfrak{g}^{\vee} \rightarrow\left(\mathfrak{P}^{1}\right)^{\vee}$ to be the restriction maps and $\mathcal{O}^{1}$ 
to be the orbit of $\bar{A}$ under the coadjoint action of $P^{1}$. Also, when $r=0$ (so $e=1$ ), we take $\left(\mathfrak{t}^{\mathrm{b}}\right)^{\prime} \subset \mathfrak{g l}_{n}(\mathbb{C})^{\vee}$ to be the set of functionals of the form $\phi(X)=\operatorname{Tr}(D X)$, where $D \in \mathfrak{t}^{b}$ is a diagonal matrix with distinct eigenvalues modulo $\mathbb{Z}$.

Definition 3.3. When $r>0$, define the extended orbit $\widetilde{\mathcal{M}}(A) \subset\left(U \backslash \mathrm{GL}_{n}(\mathbb{C})\right) \times \mathfrak{g}^{\vee}$ to be

$$
\left.\widetilde{\mathcal{M}}(A)=\left\{(U g, \alpha) \mid \pi_{\mathfrak{P}^{1}}\left(\operatorname{Ad}^{*}(g)(\alpha)\right) \in \mathcal{O}^{1}\right)\right\} .
$$

When $r=0$, we define $\widetilde{\mathcal{M}}(A)$ by

$$
\widetilde{\mathcal{M}}(A)=\left\{(g, \alpha) \in \mathrm{GL}_{n}(\mathbb{C}) \times \mathfrak{g l}_{n}(\mathbb{C})^{\vee} \mid \operatorname{Ad}^{*}(g) \alpha \in\left(\mathfrak{t}^{\mathfrak{b}}\right)^{\prime}\right\} .
$$

There is a natural action $\rho$ of $\mathrm{GL}_{n}(\mathbb{C})$ on $\widetilde{\mathcal{M}}(A)$ given by $\rho(h)(U g, \alpha)=\left(U g h^{-1}, \operatorname{Ad}^{*}(h) \alpha\right)$.

Note that when $r=0, \widetilde{\mathcal{M}}(A)$ is independent of $A$.

Proposition 3.4. 2, Proposition 5.10] The space $\widetilde{\mathcal{M}}(A)$ is a symplectic manifold, and $\rho$ is a Hamiltonian action. The moment map for $\rho$ is given by $\mu_{\rho}(U g, \alpha)=$ $\operatorname{res}(\alpha)$, where $\operatorname{res}(\alpha)=\left.\alpha\right|_{\mathfrak{g l}_{n}(\mathbb{C})}$.

Remark 3.5. If $\alpha_{\nu}$ is any representative of $\alpha$, then $\operatorname{res}(\alpha)=\operatorname{Res}\left(\alpha_{\nu} \nu\right)$.

Theorem 3.6. [2, Theorem 5.4] The moduli space $\widetilde{\mathcal{M}}(\mathbf{A})$ is the symplectic reduction of $\prod_{i \in I} \widetilde{\mathcal{M}}\left(A_{i}\right)$ by the diagonal action of $\mathrm{GL}_{n}(\mathbb{C})$ :

$$
\widetilde{\mathcal{M}}(\mathbf{A}) \cong\left(\prod_{i \in I} \widetilde{\mathcal{M}}\left(A_{i}\right)\right) / / 0 \mathrm{GL}_{n}(\mathbb{C})
$$

Moreover, $\widetilde{\mathcal{M}}(\mathbf{A})$ is a symplectic manifold.

Specifically, $\prod_{i \in I} \widetilde{\mathcal{M}}\left(A_{i}\right)$ is a symplectic manifold, and the diagonal action of $\mathrm{GL}_{n}(\mathbb{C})$ has moment map $\mu_{\mathrm{GL}_{n}}=\sum_{i \in I} \operatorname{res}_{i}$. Thus,

$$
\left(\prod_{i \in I} \widetilde{\mathcal{M}}\left(A_{i}\right)\right) / / 0 \mathrm{GL}_{n}(\mathbb{C})=\mu_{\mathrm{GL}_{n}}^{-1}(0) / \mathrm{GL}_{n}(\mathbb{C}) .
$$

In the remainder of this section, we describe a larger moduli space in which we fix only the combinatorics of $\left(V_{i}, \nabla_{i}\right)$ at $x_{i}$, and not the formal type. Again, it will be constructed as a reduction of the product of local pieces.

First, we discuss these local pieces. If $P$ is a uniform parahoric subgroup $P$ with period $e, r \geq 0$ is an integer such that $(r, e)=1$, and $T$ is a maximal torus such that $T(\mathfrak{o}) \subset P$, we let $\left(\mathfrak{P} / \mathfrak{P}^{r+1}\right)_{\text {reg }}^{\vee}$ be the set of $\gamma \in\left(\mathfrak{P} / \mathfrak{P}^{r+1}\right)^{\vee}$ such that $\left(P, r,\left.\gamma\right|_{\mathfrak{P}^{r} / \mathfrak{P}^{r+1}}\right)$ is a regular stratum centralized by $T$. We also let $\mathcal{A}(P, r)$ be the subset of $\left(\mathfrak{P} / \mathfrak{P}^{r+1}\right)_{\text {reg }}^{\vee}$ consisting of normalized formal types. Note that $\mathcal{A}(P, r)$ is the subset of $\left(\mathfrak{P} / \mathfrak{P}^{r+1}\right)_{\text {reg }}^{\vee}$ consisting of $X+H_{T}$, where $X$ is stabilized by the coadjoint action of $T(\mathfrak{o})$. We also define $\left(\mathfrak{P}^{1} / \mathfrak{P}^{r+1}\right)_{\text {reg }}^{\vee}$ to be the projection of $\left(\mathfrak{P} / \mathfrak{P}^{r+1}\right)_{\text {reg }}^{\vee}$ onto $\left(\mathfrak{P}^{1} / \mathfrak{P}^{r+1}\right)^{\vee}$.

Definition 3.7. If $r=0$, define $\widetilde{\mathcal{M}}(P, r)=\widetilde{\mathcal{M}}(A)$ for any $A$. If $r>0$, define $\widetilde{\mathcal{M}}(P, r) \subset\left(U \backslash \mathrm{GL}_{n}(\mathbb{C})\right) \times \mathfrak{g l}_{n}(\mathfrak{o})^{\vee}$ by

$$
\widetilde{\mathcal{M}}(P, r)=\left\{(U g, \alpha) \mid \pi_{\mathfrak{P}}\left(\operatorname{Ad}^{*}(g) \alpha\right) \in\left(\mathfrak{P} / \mathfrak{P}^{r+1}\right)_{\text {reg }}^{\vee}\right\} .
$$


The space $\widetilde{\mathcal{M}}(P, r)$ is a manifold; the argument is similar to the proof that $\widetilde{\mathcal{M}}(A)$ is smooth given in [2].

If we set $\mathfrak{V}=\pi_{\mathfrak{P}}^{-1}\left(\mathfrak{P} / \mathfrak{P}^{r+1}\right)_{\text {reg }}^{\vee} \subset\left(\mathfrak{g} / \mathfrak{P}^{r+1}\right)^{\vee}$, then

$$
\mathfrak{V} \times_{U} \mathrm{GL}_{n}(\mathbb{C}) \cong \widetilde{\mathcal{M}}(P, r) .
$$

The isomorphism is given by the map $(v, g) \mapsto\left(U g, \operatorname{Ad}^{*}\left(g^{-1}\right) v\right)$. Note that in the case $r=0, \mathfrak{V}=\left(\mathfrak{g} / \mathfrak{g}^{1}\right)_{\text {reg }}^{\vee}=\left(\mathfrak{t}^{b}\right)^{\prime}=\mathcal{A}\left(\mathrm{GL}_{n}(\mathfrak{o}), 0\right)$.

Proposition 3.8. There is a smooth map $\Gamma: \widetilde{\mathcal{M}}(P, r) \rightarrow \mathcal{A}(P, r)$ which assigns to $(U g, \alpha)$ the unique normalized formal type in the $P^{1}$-orbit of $\pi_{\mathfrak{P}}(\operatorname{Ad}(g)(\alpha))$. The fiber $\Gamma^{-1}(\tilde{A})$ is isomorphic to $\widetilde{\mathcal{M}}(A)$.

Proof. Using the description of $\widetilde{\mathcal{M}}(P, r)$ in (3.1), we will construct a smooth map $\zeta: \mathfrak{V} \times \mathrm{GL}_{n}(\mathbb{C}) \rightarrow \mathcal{A}(P, r)$ and then show that it $U$-equivariant.

First, we show that $\left(\mathfrak{P} / \mathfrak{P}^{r+1}\right)_{\text {reg }}^{\vee} \cong \mathcal{A}(P, r) \times_{T \cap P^{1}} P^{1} / P^{r+1}$, where $T \cap P^{1}$ acts on the right factor by left translation, and on the left factor by the coadjoint action. There is a natural map $\mathcal{A}(P, r) \times_{T \cap P^{1}} P^{1} / P^{r+1} \rightarrow\left(\mathfrak{P} / \mathfrak{P}^{r+1}\right)_{\text {reg }}^{\vee}$ given by $(Y, \bar{p}) \mapsto \operatorname{Ad}^{*}\left(p^{-1}\right) Y$. The inverse map takes a regular functional $\gamma$ to the class of $(A, \bar{p})$, where $\tilde{A}$ is the unique normalized formal type in the $P^{1}$-orbit of $\tilde{A}$ and $\tilde{A}=\operatorname{Ad}^{*}(p) \gamma$.

We now define $\zeta^{\prime}:\left(\mathfrak{P} / \mathfrak{P}^{r+1}\right)_{\mathrm{reg}}^{\vee} \rightarrow \mathcal{A}(P, r)$ as the projection onto the left factor of $\mathcal{A}(P, r) \times_{T \cap P^{1}} P^{1} / P^{r+1}$; this makes sense since the coadjoint action of $T \cap P^{1}$ on $\mathcal{A}(P, r)$ is trivial by Proposition 2.12. In particular, it is clear that $\zeta^{\prime}\left(\operatorname{Ad}^{*}(u) X\right)=$ $X$ for any $u \in U$. It follows that the map $\zeta(v, g)=\zeta^{\prime}\left(\pi_{\mathfrak{P}}(v)\right)$ is $U$-equivariant, where $U$ acts trivially on $\mathcal{A}(P, r)$. We define $\Gamma$ to be the map induced by $\zeta$ on $\mathfrak{V} \times_{U} \mathrm{GL}_{n}(\mathbb{C})$.

Finally, we see that $\widetilde{\mathcal{M}}(A)$ embeds into $\Gamma^{-1}(\tilde{A})$ by comparing Definitions 3.3 and 3.7. Moreover, if $(U g, \alpha) \in \Gamma^{-1}(\tilde{A})$, then $\pi_{\mathfrak{P}^{1}}\left(\operatorname{Ad}^{*}(g)(\alpha)\right) \in \mathcal{O}^{1}$. Therefore, $\widetilde{\mathcal{M}}(A) \cong \Gamma^{-1}(\tilde{A})$.

Before proceeding, we need to recall some facts about Poisson reduction. Recall that if a Lie group $G$ acts on a Poisson manifold $M$ via a canonical Poisson action, then there is a corresponding moment map $\mu_{M}: M \rightarrow \mathfrak{g}^{\vee}$. The following result appears in [11.

Proposition 3.9 ([11, Examples 3.B, 3.F]). Let $M$ be a Poisson manifold, and suppose that $G$ is a linear algebraic group with a free canonical Poisson action on $M$. If 0 is a regular value for $\mu_{M}$, then the Poisson structure on $M$ induces a Poisson structure on $M / /{ }_{0} G \stackrel{\text { def }}{=} \mu^{-1}(0) / G$ called the Poisson reduction of $M$. If the symplectic leaves of $M$ intersect the $G$-orbits cleanly (in the terminology of [6, II.25, p. 180]), then the symplectic leaves of $M / / 0 G$ are the connected components of the symplectic reductions of those symplectic leaves of $M$ that intersect $\mu^{-1}(0)$.

Lemma 3.10. The variety $\left(\mathfrak{P}^{1} / \mathfrak{P}^{r+1}\right)_{\text {reg }}^{\vee}$ is naturally a Poisson manifold.

Proof. Observe that $\left(\mathfrak{P}^{1} / \mathfrak{P}^{r+1}\right)_{\text {reg }}^{\vee}$ consists of those elements $X \in\left(\mathfrak{P}^{1} / \mathfrak{P}^{r+1}\right)^{\vee}$ such that $\left(P, r,\left.X\right|_{\mathfrak{P}^{r}}\right)$ is a regular stratum. Under the isomorphism $\left(\mathfrak{P}^{1} / \mathfrak{P}^{r+1}\right)^{\vee} \cong$ $\mathfrak{P}^{-r} / \mathfrak{P}$ induced by the pairing (2.1), we see that this is an open condition on the closed subvariety $Z=\left(\mathfrak{t} \cap \mathfrak{P}^{-r}+\mathfrak{P}^{-r+1}\right) / \mathfrak{P}$. By part (4) of Proposition 2.4 and 
using the notation in part (5) of the same proposition, $Z$ corresponds to the set of functionals $X$ with the property $\left.X\right|_{W_{r}}=0$. Since $\left[\mathfrak{P}^{1}, W_{r}\right] \subset \mathfrak{P}^{r+1}$, and the Poisson bracket restricted to linear functions on $\left(\mathfrak{P}^{1} / \mathfrak{P}^{r+1}\right)^{\vee}$ is just the usual Lie bracket on $\mathfrak{P}^{1} / \mathfrak{P}^{r+1}$, this implies that the ideal generated by $W_{r}$ is a Poisson ideal. It follows that $Z=\overline{\left(\mathfrak{P}^{1} / \mathfrak{P}^{r+1}\right)_{\text {reg }}^{\vee}} \subset\left(\mathfrak{P}^{1} / \mathfrak{P}^{r+1}\right)^{\vee}$ is a Poisson space, and therefore $\left(\mathfrak{P}^{1} / \mathfrak{P}^{r+1}\right)_{\text {reg }}^{\vee}$ is Poisson.

Proposition 3.11. The manifold $\widetilde{\mathcal{M}}(P, r)$ has a Poisson structure. When $r \geq 1$, the manifold $\widetilde{\mathcal{M}}(P, r)$ is isomorphic to a Poisson reduction:

$$
\widetilde{\mathcal{M}}(P, r) \cong\left(\left(\mathfrak{P}^{1} / \mathfrak{P}^{r+1}\right)_{r e g}^{\vee} \times T^{*} \mathrm{GL}_{n}(\mathbb{C})\right) / /{ }_{0} U .
$$

The symplectic leaves of $\widetilde{\mathcal{M}}(P, r)$ are the fibers of the map $\Gamma$.

Proof. In the case $r=0, \widetilde{\mathcal{M}}(P, r) \cong \widetilde{\mathcal{M}}(A)$ by the remark following Definition 3.7 Therefore, $\widetilde{\mathcal{M}}(P, r)$ is in fact symplectic.

We now suppose that $r \geq 1$. The space $\left.\left(\mathfrak{P}^{1} / \mathfrak{P}^{r+1}\right)_{\text {reg }}^{\vee} \times T^{*} \mathrm{GL}_{n}(\mathbb{C})\right)$ is a Poisson manifold using the Poisson structure of Lemma 3.10 on the first factor and the usual symplectic structure of a cotangent bundle on the second.

The group $U$ acts on $\left(\mathfrak{P}^{1} / \mathfrak{P}^{r+1}\right)_{\text {reg }}^{\vee}$ and $T^{*} \mathrm{GL}_{n}(\mathbb{C})$ via the coadjoint action and the free action induced by left multiplication on $\mathrm{GL}_{n}(\mathbb{C})$ respectively; these actions are canonical Poisson.

The moment map of the diagonal action is given by

$$
\tilde{\mu}(Y,(g, X))=-\left.\operatorname{Ad}^{*}(g)(X)\right|_{\mathfrak{u}}+\left.Y\right|_{\mathfrak{u}} .
$$

It is clear that $\tilde{\mu}$ is a submersion.

If $\tilde{\mu}(Y,(g, X))=0$, then $\left.\operatorname{Ad}^{*}(g)(X)\right|_{\mathfrak{u}}=\left.Y\right|_{\mathfrak{u}}$. Therefore, we may glue $\operatorname{Ad}^{*}(g)(X)$ and $Y$ together to obtain a functional $\psi_{Y, X} \in\left(\mathfrak{g} / \mathfrak{P}^{r+1}\right)^{\vee}$; note that $\psi_{Y, X} \in \mathfrak{V}$ if and only if $Y \in\left(\mathfrak{P}^{1} / \mathfrak{P}^{r+1}\right)_{\text {reg }}^{\vee}$ Using the description of $\widetilde{\mathcal{M}}(P, r)$ in line (3.1), we define a map $p: \tilde{\mu}^{-1}(0) \rightarrow \widetilde{\mathcal{M}}(P, r)$ by

$$
(Y,(g, X)) \mapsto\left(\psi_{Y, X}, g\right) \in\left(\mathfrak{g} / \mathfrak{P}^{r+1}\right)^{\vee} \times_{U} \mathrm{GL}_{n}(\mathbb{C}) .
$$

The map is surjective, and the fibers of $p$ are $U$-orbits.

The symplectic leaves of $\left(\mathfrak{P}^{1} / \mathfrak{P}^{r+1}\right)_{\text {reg }}^{\vee} \times T^{*} \mathrm{GL}_{n}(\mathbb{C})$ are given by $\mathcal{O} \times T^{*} \mathrm{GL}_{n}(\mathbb{C})$, where $\mathcal{O}$ is any coadjoint orbit in $\left(\mathfrak{P}^{1} / \mathfrak{P}^{r+1}\right)_{\text {reg. }}^{\vee}$. It is obvious that the $U$-orbits intersect the leaves cleanly. It now follows from Definition 3.3 and the fact that the $\widetilde{\mathcal{M}}(A)$ are connected that the symplectic leaves of $\widetilde{\mathcal{M}}(P, r)$ are given by $\widetilde{\mathcal{M}}(A)$ for $A$ a formal type corresponding to $P$ and $r$.

Lemma 3.12. The $\mathrm{GL}_{n}(\mathbb{C})$-action on $\widetilde{\mathcal{M}}(P, r)$ defined by $h(U g, \alpha)=\left(U g h^{-1}, \operatorname{Ad}^{*}(h)(\alpha)\right)$ is free canonical Poisson with submersive moment map $\mu(U g, \alpha)=\operatorname{res}(\alpha)$.

Proof. By [2, Lemma 5.12], this action restricts to a free action on each symplectic leaf $\widetilde{\mathcal{M}}(A)$. To see that it is canonical Poisson, first observe that the $\mathrm{GL}_{n}(\mathbb{C})$ action on $\left(\mathfrak{P}^{1} / \mathfrak{P}^{r+1}\right)_{\text {reg }}^{\vee} \times T^{*} \mathrm{GL}_{n}(\mathbb{C})$ given by $h \cdot(Y,(g, X))=\left(Y, g h^{-1}, A d^{*}(h) X\right)$ is canonical Poisson with moment map $(Y,(g, X)) \mapsto X$. Since it commutes with the action of $U$, it induces a canonical Poisson action on the Poisson reduction with moment map given by the same formula. It is easy to check that this action corresponds to the given action on $\widetilde{\mathcal{M}}(P, r)$ under the isomorphism of Proposition 3.11 
Since $(Y,(g, X))$ corresponds to $\left(\psi_{Y, X}, g\right)$ and $\operatorname{res}\left(\psi_{Y, X}\right)=X$, we obtain the desired expression for the moment map. Finally, by [2, Lemma 5.11], $\mu$ is even a submersion when restricted to any symplectic leaf.

We are now ready to construct the moduli space of framed connections on $\mathbb{P}^{1}(\mathbb{C})$ with fixed combinatorics. Recall that $\mathbf{x}$ is a finite set of points in $\mathbb{P}^{1}(\mathbb{C})$ indexed by $I$. Let $\mathbf{P}=\left\{P_{i}\right\}_{i \in I}$ be a collection of uniform parahoric subgroups with periods $e_{i}$ such that $P_{i} \in \mathrm{GL}_{n}\left(\mathfrak{o}_{i}\right)$, and let $\mathbf{r}=\left(r_{i}\right)_{i \in I}$ with $r_{i} \geq 0$ and $\operatorname{gcd}\left(r_{i}, e_{i}\right)=1$. Also, fix maximal tori $T_{i}$ such that $T_{i}(\mathfrak{o}) \subset P_{i}$.

It is immediate from Lemma 3.12 that the diagonal action of $\mathrm{GL}_{n}(\mathbb{C})$ on $\prod_{i \in I} \widetilde{\mathcal{M}}\left(P_{i}, r_{i}\right)$ is free canonical Poisson and that its moment map $\mu=\sum_{i \in I} \operatorname{res}_{i}$ is a submersion.

Definition 3.13. Define $\widetilde{\mathcal{M}}(\mathbf{x}, \mathbf{P}, \mathbf{r})$ as the Poisson reduction

$$
\widetilde{\mathcal{M}}(\mathbf{x}, \mathbf{P}, \mathbf{r})=\prod_{i \in I} \widetilde{\mathcal{M}}\left(P_{i}, r_{i}\right) / / \mathrm{GL}_{n}(\mathbb{C}) .
$$

We also set $\widehat{\mathcal{M}}(\mathbf{x}, \mathbf{P}, \mathbf{r})=\mu^{-1}(0)$ and $\mathcal{A}(\mathbf{x}, \mathbf{P}, \mathbf{r})=\prod_{i \in I} \mathcal{A}\left(P_{i}, r_{i}\right)$.

Proposition 3.14. There is a smooth map $\mathbf{\Gamma}: \widetilde{\mathcal{M}}(\mathbf{x}, \mathbf{P}, \mathbf{r}) \rightarrow \mathcal{A}(\mathbf{x}, \mathbf{P}, \mathbf{r})$ which assigns a normalized formal type $\tilde{A}_{i}$ to $\nabla$ at each pole $x_{i}$. The fiber $\boldsymbol{\Gamma}^{-1}(\tilde{\mathbf{A}})$ is isomorphic to $\widetilde{\mathcal{M}}(\mathbf{A})$, and the symplectic leaves of $\widetilde{\mathcal{M}}(\mathbf{x}, \mathbf{P}, \mathbf{r})$ are the connected components of these fibers.

Proof. Since the maps $\Gamma_{i}: \widetilde{\mathcal{M}}\left(P_{i}, r_{i}\right) \rightarrow \mathcal{A}\left(P_{i}, r_{i}\right)$ are $\mathrm{GL}_{n}(\mathbb{C})$-equivariant (where $\mathrm{GL}_{n}(\mathbb{C})$ acts trivially on $\left.\mathcal{A}\left(P_{i}, r_{i}\right)\right), \prod_{i \in I} \Gamma_{i}$ induces the desired map $\boldsymbol{\Gamma}$. The statement about the fibers of $\boldsymbol{\Gamma}$ follows from Proposition 3.8 and the construction of $\widetilde{\mathcal{M}}(\mathbf{A})$ in Theorem 3.6. Finally, the symplectic leaves of $\prod_{i} \widetilde{\mathcal{M}}\left(P_{i}, r_{i}\right)$ are given by $\prod_{i} \widetilde{\mathcal{M}}\left(A_{i}\right)$, and they intersect the $\mathrm{GL}_{n}(\mathbb{C})$-orbits cleanly. By Proposition 3.9. the symplectic leaves of $\widetilde{\mathcal{M}}(\mathbf{x}, \mathbf{P}, \mathbf{r})$ are the connected components of $\widetilde{\mathcal{M}}(\mathbf{A})$.

Theorem 3.15. The Poisson manifold $\widetilde{\mathcal{M}}(\mathbf{x}, \mathbf{P}, \mathbf{r})$ is isomorphic to the moduli space of isomorphism classes of triples $(V, \nabla, \mathbf{g})$, where $(V, \nabla, \mathbf{g})$ satisfies the first two conditions of Definition [3.2, and $\left(V_{i}, \nabla_{i}\right)$ contains a regular stratum of the form $\left(P_{i}, r_{i}, \beta\right)$. The manifold $\widehat{\mathcal{M}}(\mathbf{x}, \mathbf{P}, \mathbf{r})$ is isomorphic to the moduli space of isomorphism classes $(V, \nabla, \mathbf{g})$ satisfying the conditions above, with a fixed global trivialization $\phi$.

Proof. By Proposition 2.9, we may associate a unique formal type $A$ to every formal connection that contains a regular stratum $(P, r, \beta)$. Therefore, if $(V, \nabla, \mathbf{g})$ satisfies the conditions above, there is a unique element $\mathbf{A} \in \mathcal{A}\left(P_{i}, r_{i}\right)$ given by the formal type of $(V, \nabla, \mathbf{g})$ at each singular point. In particular, by Theorem 3.6, $(V, \nabla, \mathbf{g})$ corresponds to a unique point in $\widetilde{\mathcal{M}}(\mathbf{A})$. However, by Proposition 3.14, $\widetilde{\mathcal{M}}(\mathbf{A}) \cong$ $\boldsymbol{\Gamma}^{-1}(\mathbf{A})$. On the other hand, every point $p \in \widetilde{\mathcal{M}}(\mathbf{x}, \mathbf{P}, \mathbf{r})$ corresponds to a unique connection with formal type $\boldsymbol{\Gamma}(p)$.

Now, suppose $q=\left(U_{i} g_{i}, \alpha_{i}\right)_{i \in I} \in \widehat{\mathcal{M}}(\mathbf{x}, \mathbf{P}, \mathbf{r})$. Fix a global form $\nu \in \Omega_{\mathbb{P}^{1}}^{1}$. By (2.1), we may associate to $\alpha_{i}$ a unique meromorphic form $\alpha_{i \nu} \nu$ with coefficients in $\mathfrak{g l}_{n}\left(F_{i}\right)$. Since $\mu(q)=0, \sum_{i \in I} \operatorname{Res}_{i}\left(\alpha_{i \nu} \nu\right)=0$. It follows that $q$ determines a global form $\alpha \nu$, and thus a global meromorphic connection $\nabla=d+\alpha \nu$ on the trivial rank $n$ vector bundle over $\mathbb{P}^{1}$. It is easily checked that this gives a bijection between points in $\widehat{\mathcal{M}}(\mathbf{x}, \mathbf{P}, \mathbf{r})$ and triples $(V, \nabla, \mathbf{g})$. 


\section{INTEGRABLE DEFORMATIONS}

Let $X=\mathbb{P}^{1}(\mathbb{C})$, and let $V$ be an $n$-dimensional trivial vector bundle on $X$. In this section, we will consider the deformations of a connection $(V, \nabla)$ which contains a regular stratum at each singularity.

4.1. Formal Deformations. Without loss of generality, take $x_{1}=0$ and fix a parameter $z$ at 0 . We will suppress the subscripts on $\left(P_{i}, r_{i}, \beta_{i}\right)$, etc. when we work locally at 0 . Let $F$ be the field of Laurent series at 0 and $\mathfrak{o} \subset F$ the ring of power series. Define $\hat{V}$ to be the formal completion of $V$ at 0 , and let $\hat{\nabla}$ be the induced formal connection.

Now, let $D=\operatorname{Spec}(\mathfrak{o})$, and fix a standard parahoric $P$ and an integer $r$ with $\operatorname{gcd}(r, e)=1$. We also fix a torus $T$, with $T(\mathfrak{o}) \subset P$, as in (2.2). Let $\Delta$ be an analytic polydisk; we denote its ring of functions by $R$. A formal flat deformation is a flat, meromorphic connection $(\bar{V}, \bar{\nabla})$ on $D \times \Delta$ satisfying the following properties:

- the vector bundle $\bar{V}$ is isomorphic to the trivial rank $n$ vector bundle, and

- the restriction of $\bar{\nabla}$ to $D \times\{y\}$, denoted by $\hat{\nabla}_{y}$, contains a regular stratum $\left(P_{y}, r, \beta_{y}\right)$.

Fix a trivialization $\phi$ of $\bar{V}$ so that we may identify all other trivializations with elements of $\mathrm{GL}_{n}(\mathfrak{o} \otimes R)$. We say that the deformation $(\bar{V}, \bar{\nabla})$ is framed if there exists a trivialization $g \in \mathrm{GL}_{n}(R)$ with the property that $P_{y}=P^{g(y)}, \beta_{y}=\beta^{g(y)}$, and the regular stratum $\left(P_{y}, r, \beta_{y}\right)$ is centralized by $T_{y}:=T^{g(y)}$. In particular, any representative $\left(\beta_{y}\right)_{\nu} \in \mathfrak{P}_{y}^{-r}$ for $\beta_{y}$ lies in $\mathfrak{t}_{y}+\mathfrak{P}_{y}^{-r+1}$ by [2, Remark 3.5] (with $\operatorname{ord}(\nu)=-1)$.

We denote $\mathfrak{P}_{\Delta}^{\ell}=\mathfrak{P}^{\ell} \otimes R, \overline{\mathfrak{P}}_{\Delta}^{\ell}=\mathfrak{P}_{\Delta}^{\ell} / \mathfrak{P}_{\Delta}^{\ell+1}$, and $\mathfrak{t}_{\Delta}=\mathfrak{t} \otimes R$. We define $P_{\Delta}$ and $T_{\Delta}$ similarly. Suppose that $(\bar{V}, \bar{\nabla})$ is framed by $g$, and fix a nonzero one-form $\nu$ at 0 . If $\nu=u \frac{d z}{z}$, write $\frac{z \nu}{d z}=u$. Let $A(y)$ be the formal type of $\bar{\nabla}$ at $y$. Using the pairing in (2.1), we may choose a representative $A_{\nu}(y)$ for $A(y)$ of the form

$$
A_{\nu}(y)=\left(\frac{-r}{n} t_{-r}(y) \varpi_{T}^{-r}+\ldots+\frac{-1}{n} t_{-1}(y) \varpi_{T}^{-1}+t_{0}(y)\right) \frac{d z}{z \nu}
$$

with $t_{i}(y) \in \mathfrak{t}_{\Delta}^{b}$. For example, $A_{\frac{d z}{z}}=\left(\frac{-r}{n} t_{-r}(y) \varpi_{T}^{-r}+\ldots+\frac{-1}{n} t_{-1}(y) \varpi_{T}^{-1}+t_{0}(y)\right)$.

Recall that any element of $\mathfrak{t}$ can be written as a Laurent series $t=\sum_{i=-N}^{\infty} t_{j} \varpi_{T}^{j}$ with $t_{i} \in \mathfrak{t}^{b}$. We define an endomorphism $\delta_{e}$ of $\mathfrak{t}$ via $\delta_{e}(t)=\sum_{i=-N}^{\infty} \frac{i}{e} t_{i} \varpi_{T}^{i}$.

Lemma 4.1. Suppose $t \in \mathfrak{t}_{\Delta}$. Then, $z \partial_{z} t-\left[t, H_{T}\right]=\delta_{e} t$. Moreover, any solution $B \in \Omega_{\Delta}^{1}\left(\left(\mathfrak{t}+\mathfrak{P}^{\ell}\right) / \mathfrak{P}^{\ell}\right)$ to the differential equation $z \partial_{z} B-\left[B, H_{T}\right]=\delta_{e} t+t_{0}+\mathfrak{P}^{\ell}$ has the form $B=t+f+\mathfrak{P}^{\ell}$, where $f \in \mathfrak{t}_{\Delta}^{b}$. There is no solution when $\ell \geq 0$ and $t_{0} \neq 0$.

Proof. Since the equations are block diagonal in $\mathfrak{t}$, we may immediately reduce to the case where $T=E^{\times}$. A direct calculation shows that $z \partial_{z} \varpi_{T}^{i}-\left[\varpi_{T}, H_{E}\right]=$ $\frac{i}{e} \varpi_{T}^{i}=\delta_{e}\left(\varpi_{T}^{i}\right)$. This proves the first statement. The second follows by applying the same calculation to each term of $B$ up to $\varpi_{T}^{\ell}$. Note that $z \partial_{z} \varpi_{T}^{0}-\left[\varpi_{T}^{0}, H_{E}\right]=0$, so there is no solution when $t_{0} \neq 0$.

As in the previous section, we will let $p \cdot[\bar{\nabla}]_{g}$ denote the $d_{z}$ part of the gauge transformation formula: $p \cdot[\bar{\nabla}]_{g}=\operatorname{Ad}(g)\left([\bar{\nabla}]_{g}\right)-\left(d_{z} p\right) p^{-1}$. We will use $d_{\Delta}$ and $\bar{d}$ 
to denote the exterior differential on $\Delta$ and $\mathbb{P}^{1} \times \Delta$, respectively. Suppose that $\tilde{A}_{\nu}$ is the normalized formal type associated to $A_{\nu}$. By Proposition 2.11, there exists an element $p \in P_{\Delta}$ such that

$$
p(y) \cdot\left[\hat{\nabla}_{y}\right]_{g}=\tilde{A}_{\nu}(y) \nu .
$$

Let $d_{\Delta}$ be the exterior differential on $\Delta$. Define

$$
A_{\Delta}(y)=\sum_{i=-r}^{-1} \varpi_{T}^{-i} d_{\Delta} t_{i},
$$

so that $d_{\Delta} A_{\frac{d z}{z}}=\delta_{e} A_{\Delta}+d_{\Delta} t_{0}$.

Proposition 4.2. Let $p$ be as above. Then, $p \cdot[\bar{\nabla}]_{g}-\left(d_{\Delta} p\right) p^{-1}=\tilde{A}_{\nu} \nu+A_{\Delta}+f$, where $f \in \Omega_{\Delta}^{1}\left(\mathfrak{t}^{b}\right)$ is closed. Moreover, $t_{0}(y)$ must be constant. Conversely, if $q \in$ $\mathrm{GL}_{n}(F \otimes R), f$ is closed, and $t_{0}(y)$ is constant, then $\left[\bar{\nabla}^{\prime}\right]=q^{-1} \cdot\left(\tilde{A}_{\nu} \nu+A_{\Delta}+f\right)+$ $q^{-1}\left(d_{\Delta} q\right)$ determines a flat meromorphic connection $\bar{\nabla}^{\prime}$ on $\bar{V}$.

Remark 4.3. The case $e=1$ is proved in the Appendix of [1].

Proof. Note that the connection determined by $p \cdot[\bar{\nabla}]_{g}-\left(d_{\Delta} p\right) p$ is flat, since $\bar{\nabla}$ is flat and $-\left(d_{\Delta} p\right) p$ is simply the $d_{\Delta}$ part of the gauge transformation formula. Conversely, if the connection determined by $\tilde{A}_{\nu} \nu+A_{\Delta}+f$ is flat, then $\bar{\nabla}^{\prime}$ is flat by the same argument.

Without loss of generality, set $\nu=\frac{d z}{z}$. It suffices to show that whenever $[\bar{\nabla}]=$ $\tilde{A}_{\frac{d z}{z}} \frac{d z}{z}+B$ for some $B \in \Omega_{\Delta}^{1}\left(\mathfrak{g l}_{n}(F)\right)$, then $\bar{\nabla}$ is flat if and only if $B$ has the form $A_{\Delta}+f$. We observe that $\bar{\nabla}$ is flat if and only if it satisfies the conditions

$$
\begin{gathered}
d_{\Delta}\left(A_{\frac{d z}{z}}\right)-z \partial_{z} B+\left[B, A_{\frac{d z}{z}}+H_{T}\right]=0 \\
\text { and } d_{\Delta} B+B \wedge B=0 .
\end{gathered}
$$

If $B=A_{\Delta}+f$, (4.2) holds trivially while (4.1) follows from the first part of Lemma 4.1, so $\bar{\nabla}$ is flat.

We now prove the converse. If $r=0$, then $e=1$ and $H_{T}=0$. In this case, we may take $A_{\frac{d z}{z}}=t_{0}(y)$ to be a regular diagonal matrix with entries in $R$; moreover, no two eigenvalues of $A_{\underline{d z}}$ differ by an integer. Setting $B=\sum B_{\ell} z^{\ell}$ with $B_{\ell} \in$ $\mathfrak{g l}_{n}(\mathbb{C})$, (4.2) reduces to $\left[B_{\ell}, A_{\frac{d z}{z}}\right]=\ell B_{\ell}$ when $\ell \neq 0$. The eigenvalue condition now implies that $B_{\ell}=0$. whenever $\ell \neq 0$, by the condition on the eigenvalues of $A_{\frac{d z}{z}}$. On the other hand, $\left[B_{0}, A_{\frac{d z}{z}}\right]=-d_{\Delta}\left(A_{\frac{d z}{z}}\right)$. Since the right hand side is a diagonal matrix, both sides must be identically 0 . It follows that $d_{\Delta}\left(A_{\frac{d z}{z}}\right)=0$ and $B_{0} \in \Omega_{\Delta}^{1}\left(\mathfrak{t}^{\mathfrak{b}}\right)$ while the fact that $B_{0}$ is closed follows from (4.2).

We now consider the case $r \geq 1$. In the following, let $t_{0}(y)$ be the constant term of $A_{\frac{d z}{z}}$. Suppose, by induction, that $B \in A_{\Delta}+f+\Omega_{\Delta}^{1}\left(\mathfrak{t}^{\mathfrak{b}}+\mathfrak{P}^{\ell}\right)$. (Note that $B \in \Omega^{1}\left(\mathfrak{P}^{L}\right)$ for some $L \leq-r$, so the first inductive step is trivially satisfied for $\ell=L)$. Applying the first part of Lemma 4.1 with $t=A_{\Delta}$ gives

$$
d_{\Delta}\left(A_{\frac{d z}{z}}\right)+\left[B, H_{T}\right]-z \partial_{z} B \in d_{\Delta} t_{0}+\Omega_{\Delta}^{1}\left(\mathfrak{P}^{\ell}\right) .
$$

We deduce from (4.1) that $\left[B, A_{\frac{d z}{z}}\right] \in d_{\Delta} t_{0}+\Omega_{\Delta}^{1}\left(\mathfrak{P}^{\ell}\right)$.

When $\ell<1, d_{\Delta} t_{0} \in \Omega_{\Delta}^{1}\left(\mathfrak{P}^{\ell}\right)$. This implies that $\left[B, A_{\frac{d z}{z}}\right] \in \Omega_{\Delta}^{1}\left(\mathfrak{P}^{\ell}\right)$, so $B \in$ $\Omega_{\Delta}^{1}\left(\mathfrak{t}+\mathfrak{P}^{\ell+r}\right)$. Next, consider $\ell=1$. By the $\ell=0$ step, we know that $B \in \Omega_{\Delta}^{1}(\mathfrak{t}+$ 
$\left.\mathfrak{P}^{r}\right)$. Part (3) of Proposition 2.4 shows that $\left[B, A_{\frac{d z}{z}}\right]+\Omega_{\Delta}^{1}\left(\mathfrak{P}^{1}\right) \in \operatorname{ker}\left(\bar{\pi}_{\mathfrak{t}} \otimes \mathrm{I}_{\Omega_{\Delta}^{1}}\right)$. Since $d_{\Delta} t_{0} \in \Omega_{\Delta}^{1}\left(\mathfrak{t}^{b}\right)$, part (1) of the same proposition gives $d_{\Delta} t_{0} \in \Omega_{\Delta}^{1}\left(\mathfrak{P}^{1}\right)$. Since $\mathfrak{t}^{b} \cap \mathfrak{P}^{1}=\{0\}$, we see that $d_{\Delta} t_{0}=0$. Thus, we may conclude that the inductive hypothesis implies that $\left[B, A_{\frac{d z}{z}}\right] \in \Omega_{\Delta}^{1}\left(\mathfrak{P}^{\ell}\right)$ for $\ell \geq 1$ as well. As before, $B \in$ $\Omega_{\Delta}^{1}\left(\mathfrak{t}+\mathfrak{P}^{\ell+r}\right) \subset \Omega_{\Delta}^{1}\left(\mathfrak{t}+\mathfrak{P}^{\ell+1}\right)$.

To complete the inductive step, we apply the second part of Lemma 4.1 to obtain $B \in A_{\Delta}+\Omega_{\Delta}^{1}\left(\mathfrak{t}^{\mathfrak{b}}+\mathfrak{P}^{\ell+1}\right)$. Using the fact that the sum $\mathfrak{t}^{\mathfrak{b}}+\mathfrak{P}^{\ell}$ is direct for $\ell \geq 1$, we actually obtain $f \in \Omega_{\Delta}^{1}\left(\mathfrak{t}^{b}\right)$ such that $B \in A_{\Delta}+f+\Omega_{\Delta}^{1}\left(\mathfrak{P}^{\ell}\right)$ for all $\ell$. The result now follows since $\bigcap_{\ell} \mathfrak{P}^{\ell}=0$.

Definition 4.4. We say that a compatible framing $g$ for $\bar{\nabla}$ is good if there exists $p \in P_{\Delta}^{1}$ such that $p \cdot[\bar{\nabla}]_{g}-\left(d_{\Delta} p\right) p^{-1}=\tilde{A}_{\nu} \nu+A_{\Delta}$.

Proposition 4.5. Every framed flat deformation has a good compatible framing.

Proof. If $g$ is a compatible framing, there exists $p \in P$ such that $p \cdot\left([\bar{\nabla}]_{g}\right)-$ $\left(d_{\Delta} p\right) p^{-1}=\left(A_{\frac{d z}{z}}+H_{T}\right) \frac{d z}{z}+A_{\Delta}+f$ by Proposition 4.2. Since $f$ is closed on $\Delta$, it is exact. Choosing $\varphi \in \mathfrak{t}_{\Delta}^{b}$ such that $f=d_{\Delta} \varphi$, we obtain

$$
\operatorname{Ad}\left(e^{\varphi}\right)\left(p \cdot\left([\bar{\nabla}]_{g}\right)-d_{\Delta}(p) p^{-1}\right)-d_{\Delta} \varphi=\left(A_{\frac{d z}{z}}+H_{T}\right) \frac{d z}{z}+A_{\Delta} .
$$

It follows that $e^{\varphi} g$ is a good compatible framing.

\subsection{Global Deformations.}

Definition 4.6. A framed global deformation is a triple $(\mathbf{g}, \bar{V}, \tilde{\nabla})$ consisting of:

(1) a trivializable rank $n$ vector bundle $\bar{V}$ on $\mathbb{P}^{1} \times \Delta$;

(2) an $R$-relative connection $\tilde{\nabla}$;

(3) a collection of analytic framings $\mathbf{g}=\left(g_{i}\right)_{i \in I}, g_{i}: \Delta \rightarrow U_{i} \backslash \mathrm{GL}_{n}(\mathbb{C})$;

(4) the restriction of $(\bar{V}, \tilde{\nabla})$ to $\mathbb{P}^{1} \times\{y\}$ must lie in $\widetilde{\mathcal{M}}(\mathbf{x}, \mathbf{P}, \mathbf{r})$ with compatible framing $\mathbf{g}(y)$.

We say that a framed deformation is integrable if there exists a total flat connection $\bar{\nabla}$ on $\mathbb{P}^{1} \times \Delta$ with $\mathbb{P}^{1}$ part $\tilde{\nabla}$.

We note that $(\mathbf{g}, \bar{V}, \tilde{\nabla})$ determines a smooth map $\Delta \rightarrow \widetilde{\mathcal{M}}(\mathbf{x}, \mathbf{P}, \mathbf{r})$. Specifically, there are maps $g_{i}(y)$ and $\alpha_{i}(y)$ such that the connection on the fiber above $y$ corresponds to the point $\left(U_{i} g_{i}(y), \alpha_{i}(y)\right)_{i \in I} \in \widetilde{\mathcal{M}}(\mathbf{x}, \mathbf{P}, \mathbf{r})$.

Suppose that $(\mathbf{g}, \bar{V}, \tilde{\nabla})$ is an integrable framed global deformation. If we fix a trivialization for $\bar{V}$, we may write $[\bar{\nabla}]=\alpha \nu+\Upsilon$, where $\Upsilon$ is a section of $\Omega_{\Delta / \mathbb{C}}^{1}(\operatorname{End}(\bar{V}))$ with poles along $\left\{x_{i}\right\}$. The curvature of $\bar{\nabla}$ is given by

$$
\Xi(\alpha, \Upsilon)=\bar{d}(\alpha \nu+\Upsilon)+\Upsilon \wedge \alpha \nu+\alpha \nu \wedge \Upsilon+\Upsilon \wedge \Upsilon \in \Omega_{\Delta \times \mathbb{P}^{1}}^{2}
$$

Thus, $\bar{\nabla}$ is flat if and only if the following hold:

$$
\tau_{\nu} \Upsilon=d_{\Delta} \alpha+[\Upsilon, \alpha] \quad \text { and } \quad 0=d_{\Delta} \Upsilon+\Upsilon \wedge \Upsilon
$$

An integrable deformation $(\mathbf{g}, \bar{V}, \tilde{\nabla})$ determines a flat formal deformation $\left(\hat{V}_{i}, \bar{\nabla}_{i}\right)$ at each singular point. Therefore, if $\tilde{A}_{i}(y)$ is the normalized formal type of $\left(\hat{V}_{i}, \bar{\nabla}_{i}\right)$ at $y \in \Delta$, Proposition 4.5 implies that there exists $p_{i} \in P_{i}^{1}$ such that $p_{i} g_{i} \cdot[\bar{\nabla}]-$ 
$\operatorname{Ad}\left(p_{i}\right)\left(d_{\Delta} g_{i} g_{i}^{-1}\right)-\left(d_{\Delta} p_{i}\right) p_{i}^{-1}=\tilde{A}_{i, \nu} \nu+A_{i, \Delta}$. Since $p_{i}^{-1} d_{\Delta} p_{i} \in \Omega_{\Delta}^{1}\left(\mathfrak{P}^{1}\right)$ by [2, Lemma 4.4], we deduce

$$
\Upsilon \in \operatorname{Ad}\left(g_{i}^{-1} p_{i}^{-1}\right) A_{i, \Delta}+g_{i}^{-1} d_{\Delta} g_{i}+\Omega_{\Delta}^{1}\left(\left(\mathfrak{P}_{i}^{1}\right)^{g_{i}}\right)
$$

Set $\mathbf{r}_{\mathbf{e}}=\left(r_{i}^{\prime}\right)_{i \in I}:=\left(\left\lceil\frac{r_{i}}{e_{i}}\right\rceil\right)_{i \in I}$, where $\left\lceil\frac{r_{i}}{e_{i}}\right\rceil$ is the integer ceiling of $\frac{r_{i}}{e_{i}}$. Let $D_{\mathbf{r}}$ be the divisor $\sum_{i \in I} r_{i}^{\prime}\left[x_{i}\right]$ on $\mathbb{P}^{1}$.

Definition 4.7. Let $\mathfrak{g l}_{n \mathbb{P}^{1}}$ denote the trivial $\mathfrak{g l}_{n}$-bundle on $\mathbb{P}^{1}$, and let $\mathfrak{g l}_{n \mathbb{P}^{1}}^{\mathrm{r}_{\mathbf{e}}}=$ $\mathfrak{g l}_{n \mathbb{P}^{1}}\left(D_{\mathbf{r}}\right)$ be the sheaf corresponding to the divisor $D_{\mathbf{r}}$

Note that sections of $\mathfrak{g l}_{n \mathbb{P}^{1}}^{\mathbf{r}_{\mathbf{e}}}$ have poles of order at most $r_{i}^{\prime}$ at $x_{i}$.

Fix a set of parameters $\left(z_{i}\right)_{i \in I}$ at each singular point $x_{i}$ with the property that each $z_{i}$ has a pole at a fixed point $x_{0}$. Define a $\mathbb{C}$-linear map $\phi_{i}: \mathfrak{g l}_{n}\left(F_{i}\right) \rightarrow \mathfrak{g l}_{n}(\mathbb{C})$ by $\phi_{i}(X)=\operatorname{Res}_{x_{i}}\left(X \frac{d z_{i}}{z_{i}}\right)$. Thus, $\phi_{i}$ extracts the constant term of $X$ at $x_{i}$ with respect to $z_{i}$. This induces a map $\bar{\phi}_{i}: \mathfrak{g l}_{n}\left(F_{i}\right) / \mathfrak{P}_{i}^{1} \rightarrow \mathfrak{g l}_{n}(\mathbb{C}) / \mathfrak{u}_{i}$.

Next, we define a map $\varsigma$ which assigns a global section of $\mathfrak{g l}_{n \mathbb{P} 1}^{\mathbf{r}_{\mathbf{e}}}$ to a collection of principal parts at $\mathbf{x}$. Given $X_{i} \in \mathfrak{g}_{i}^{-r_{i}^{\prime}} / \mathfrak{g}_{i}$, let $\tilde{X}_{i} \in H^{0}\left(\mathbb{P}^{1} ; \mathfrak{g l}_{n \mathbb{P}^{1}}^{\mathbf{r}_{\mathbf{e}}}\right)$ be the section corresponding to the unique lift of $X_{i}$ to $\mathfrak{g l}_{n}\left(z_{i}^{-1} \mathbb{C}\left[z_{i}^{-1}\right]\right)$.

Definition 4.8. The map $\varsigma: \prod_{i \in I} \mathfrak{g}_{i}^{-r_{i}^{\prime}} / \mathfrak{g}_{i} \rightarrow H^{0}\left(\mathbb{P}^{1} ; \mathfrak{g l}_{n \mathbb{P}^{1}}^{\mathbf{r}_{\mathbf{e}}}\right)$ is given by

$$
\varsigma\left(\left(X_{i}\right)_{i \in I}\right)=\sum_{i \in I} \tilde{X}_{i} .
$$

We will usually write $X^{0}$ for $\varsigma\left(\left(X_{i}\right)_{i \in I}\right)$.

Remark 4.9. This map commute with the adjoint action of $\mathrm{GL}_{n}(\mathbb{C})$, i.e., $\operatorname{Ad}(g)\left(X^{0}\right)=$ $(\operatorname{Ad}(g) X)^{0}$ for any $g \in \mathrm{GL}_{n}(\mathbb{C})$. Indeed, $\operatorname{Ad}(g)\left(\tilde{X}_{i}\right)=\widehat{\operatorname{Ad}(g) X_{i}}$ for each $i$ since $\operatorname{Ad}(g)$ stabilizes $\mathfrak{g l}_{n}\left(z_{i}^{-1} \mathbb{C}\left[z_{i}^{-1}\right]\right)$.

We are now ready to describe a system of differential equations satisfied by an integrable deformation. Let $(\bar{V}, \bar{\nabla})$ be a deformation of $(V, \nabla)$ as in Definition 4.6. corresponding to a map $\left(g_{i}(y), \alpha_{i}(y)\right)_{i \in I}$ from $\Delta$ to $\widetilde{\mathcal{M}}(\mathbf{x}, \mathbf{P}, \mathbf{r})$.

Fix a uniformizer $z_{0}$ at $x_{0}$.

Lemma 4.10. If $\Upsilon^{\prime}$ has principal part $\Upsilon_{i}$ at each $x_{i}$ and is holomorphic elsewhere, then $\Upsilon^{\prime}-\Upsilon^{0}=\left.\Upsilon^{\prime}\right|_{z_{0}=0}$.

Proof. Since $\Upsilon^{\prime}$ and $\Upsilon^{0}$ have the same principal parts at each singular point, $\Upsilon^{\prime}-$ $\Upsilon^{0}=X \in \Omega_{\Delta}^{1}\left(\mathfrak{g l}_{n}(\mathbb{C})\right)$. By construction, $\Upsilon^{0}$ and $\Upsilon^{\prime}$ are holomorphic at $x_{0}$, and $\left.\Upsilon^{0}\right|_{z_{0}=0}=0$. Therefore, $X=\left.\Upsilon^{\prime}\right|_{z_{0}=0}$.

Lemma 4.11. Given $\Upsilon \in \Omega_{\Delta}^{1}\left(H^{0}\left(\mathbb{P}^{1} ; \mathfrak{g l}_{n \mathbb{P}^{1}}^{\mathbf{r}_{\mathbf{e}}}\right)\right)$, define $\Upsilon_{i}=\Upsilon+\mathfrak{g}_{i}$. Any integrable framed deformation $\bar{\nabla}$ is $G L_{n}(R)$-gauge equivalent to a deformation $\alpha \nu+\Upsilon$ satisfying $\Upsilon=\varsigma\left(\left(\Upsilon_{i}\right)_{i \in I}\right)$.

Proof. Suppose that $[\bar{\nabla}]=\alpha^{\prime} \nu+\Upsilon^{\prime}$. Then, $\Upsilon^{\prime}-\left(\Upsilon^{\prime}\right)^{0}=X \in \Omega_{\Delta}^{1}\left(\mathfrak{g l}_{n}(\mathbb{C})\right)$ by Lemma 4.10. Since the image of $\varsigma$ is closed under conjugating by $\operatorname{GL}_{n}(R)$, it suffices to show that there exists $g \in \mathrm{GL}_{n}(R)$ such that $\Upsilon:=\operatorname{Ad}(g) \Upsilon^{\prime}-\left(d_{\Delta} g\right) g^{-1}=$ $\operatorname{Ad}(g)\left(\Upsilon^{\prime}\right)^{0}$.

The system of differential equations $g^{-1}\left(d_{\Delta} g\right)=X$ has a solution if $d_{\Delta} X+X \wedge$ $X=0$. To see this, recall that $d_{\Delta} X=\left.d_{\Delta} \Upsilon^{\prime}\right|_{z_{0}=0}$. By flatness, $d_{\Delta} \Upsilon^{\prime}+\Upsilon^{\prime} \wedge \Upsilon^{\prime}=0$, so $d_{\Delta} X+X \wedge X=\left.\left(d_{\Delta} \Upsilon^{\prime}+\Upsilon^{\prime} \wedge \Upsilon^{\prime}\right)\right|_{z_{0}=0}=0$. 
Now, suppose that $(\mathbf{g}, \bar{V}, \tilde{\nabla})$ is a deformation. Choose $p_{i} \in P_{\Delta}^{1}$ such that $p_{i} \cdot \alpha \nu=$ $\tilde{A}_{i \nu} \nu$, and write $\hat{g}_{i}=p_{i} g_{i}$. Let $\Upsilon_{i}=\operatorname{Ad}\left(\hat{g}_{i}^{-1}\right) A_{i, \Delta}+\Omega_{\Delta}^{1}\left(\mathfrak{g}_{i}\right)$, with corresponding global section $\Upsilon^{0} \in \Omega_{\Delta}^{1}\left(H^{0}\left(\mathbb{P}^{1} ; \mathfrak{g l}_{n \mathbb{P}^{1}}^{\mathrm{r}_{\mathrm{e}}}\right)\right)$.

Theorem 4.12. A good framed deformation $(\mathrm{g}, \bar{V}, \tilde{\nabla})$ is integrable if and only if it is $\mathrm{GL}_{n}(R)$-gauge-equivalent to a deformation satisfying the following equations:

(1) $\phi_{i}\left(\operatorname{Ad}\left(p_{i}^{-1}\right)\left(A_{i, \Delta}\right)+\left(d_{\Delta} g_{i}\right) g_{i}^{-1}\right) \in \phi_{i}\left(\operatorname{Ad}\left(g_{i}\right) \Upsilon^{0}\right)+\Omega_{\Delta}^{1}\left(\mathfrak{u}_{i}\right)$;

(2) $d_{\Delta} \alpha \in \tau_{\nu} \Upsilon^{0}+\left[\alpha, \Upsilon^{0}\right]+\Omega_{\Delta}^{1}\left(\mathfrak{g}_{i} \frac{d z_{i}}{z_{i}}\right)$; and

(3) $d \Upsilon^{0}+\Upsilon^{0} \wedge \Upsilon^{0}=0$.

Proof. Suppose that $(\mathrm{g}, \bar{V}, \tilde{\nabla})$ is integrable. By Lemma 4.11, there exists a gauge $g \in \mathrm{GL}_{n}(R)$ such that $[\bar{\nabla}]_{g}=\alpha \nu+\Upsilon$ with $\Upsilon=\Upsilon^{0}$. By Proposition 4.2.

$$
\Upsilon \in \operatorname{Ad}\left(\hat{g}_{i}^{-1}\right) A_{i, \Delta}+\hat{g}_{i}^{-1} d_{\Delta} \hat{g}_{i}+\left(\mathfrak{P}_{i, \Delta}^{1}\right)^{g_{i}} .
$$

Therefore, condition (11) is satisfied by applying $\operatorname{Ad}\left(g_{i}\right)$ and $\phi_{i}$. Moreover, $\alpha \nu+\Upsilon$ is flat, so conditions (2) and (3) follow from (4.3).

To see the converse, note that condition (11) shows that $\Upsilon^{0}$ satisfies (4.6). Condition (2) implies that the cross-term of the curvature of $\alpha \nu+\Upsilon^{0}$ is zero modulo $\mathfrak{g}_{i}^{1} \frac{d z_{i}}{z_{i} \nu}$. Writing this term as $\sum f_{j} d y_{j}$, where the $d y_{j}$ 's are a basis for $\Omega_{\Delta}^{1}$, we see that $f_{j} \in H^{0}\left(\mathbb{P}^{1} ; \Omega_{\mathbb{P}^{1}}^{1}\right)=\{0\}$. Since the term in $\Omega_{\Delta}^{2}$ vanishes by condition (3), we see that the curvature vanishes.

Corollary 4.13. A good framed deformation $(\mathrm{g}, \bar{V}, \tilde{\nabla})$ is integrable if and only if there exists $g \in \mathrm{GL}_{n}(R)$ such that

(1) $\phi_{i}\left(\operatorname{Ad}\left(g_{i}\right)\left(g^{-1} d_{\Delta} g+\Upsilon^{0}\right)\right) \in \phi_{i}\left(\operatorname{Ad}\left(p_{i}^{-1}\right) A_{\Delta, i}+\left(d_{\Delta} g_{i}\right) g_{i}^{-1}\right)+\Omega_{\Delta}^{1}\left(\mathfrak{u}_{i}\right)$;

(2) $d_{\Delta} \alpha \in \tau_{\nu} \Upsilon^{0}+\left[\alpha, \Upsilon^{0}+g^{-1} d_{\Delta} g\right]+\Omega_{\Delta}^{1}\left(\mathfrak{g}_{i}^{1} \frac{d z_{i}}{z_{i} \nu}\right)$; and

(3) $d_{\Delta}\left(\Upsilon^{0}+g^{-1} d_{\Delta} g\right)+\left(\Upsilon^{0}+g^{-1} d_{\Delta} g\right) \wedge\left(\Upsilon^{0}+g^{-1} d_{\Delta} g\right)=0$.

Proof. Set $[\bar{\nabla}]=\alpha \nu+\Upsilon$ and choose $g \in \mathrm{GL}_{n}(R)$ such that $[\bar{\nabla}]_{g}=\alpha^{\prime} \nu+\Upsilon^{\prime}$ with $\Upsilon^{\prime}=\left(\Upsilon^{\prime}\right)^{0}$. The proof above shows that $\left(\mathbf{g}^{\prime}, \alpha^{\prime}, \Upsilon^{\prime}\right)$ satisfy the conditions in the theorem. One obtains the equations in the corollary by substituting $\Upsilon^{\prime}=\operatorname{Ad}(g) \Upsilon^{0}$, $\alpha^{\prime}=\operatorname{Ad}(g)(\alpha)$, and $g_{i}^{\prime}=g_{i} g^{-1}$. The converse is proved similarly.

Remark 4.14. It will be shown later in Theorem 5.7 that the third condition in these two results is unnecessary.

\section{INTEGRABILITY}

In this section we show that the equations from Corollary 4.13 determine a Frobenius integrable system on $\widetilde{\mathcal{M}}(\mathbf{x}, \mathbf{P}, \mathbf{r})$. Throughout, we will fix a global meromorphic one-form $\nu$ on $\mathbb{P}^{1}(\mathbb{C})$.

5.1. The Differential Ideal. We will construct a differential ideal $\mathcal{I}_{\mathrm{GL}_{n}}$ on $\widehat{\mathcal{M}}(\mathbf{x}, \mathbf{P}, \mathbf{r})$ corresponding to the system of differential equations in Corollary 4.13 , Throughout, we will simplify notation by suppressing $\mathbf{x}, \mathbf{P}$, and $\mathbf{r}$ in the notation and using $d$ for the exterior differential on all spaces whenever there is no risk of ambiguity. We will also write $\widetilde{\mathcal{M}}_{i}=\widetilde{\mathcal{M}}\left(P_{i}, r_{i}\right)$ and $\mathcal{A}_{i}=\mathcal{A}\left(P_{i}, r_{i}\right)$. 
Set $\mathfrak{t}_{i}^{j}=\mathfrak{t}_{i} \cap \mathfrak{P}_{i}^{j}$ and $T_{i}^{j}=T_{i} \cap P_{i}^{j}$. First, we define maps $\hat{\Gamma}_{i}: \widehat{\mathcal{M}} \rightarrow \mathcal{A}_{i}$ and $\hat{\Gamma}_{i \nu}: \widehat{\mathcal{M}} \rightarrow\left(\mathfrak{t}_{i}^{-r_{i}}+H_{T}\right) \frac{d z_{i}}{z_{i} \nu}:$

$$
\hat{\Gamma}_{i}\left(\left(U_{j} g_{j}, \alpha_{j}\right)_{i \in I}\right)=\Gamma_{i}\left(U_{i} g_{i}, \alpha_{i}\right)
$$

and $\hat{\Gamma}_{i \nu}\left(\left(U_{j} g_{j}, \alpha_{j}\right)\right)$ is the standard representative of $\Gamma_{i}\left(U_{i} g_{i}, \alpha_{i}\right)$ in $\mathfrak{P}_{i}^{-r} \frac{d z_{i}}{z_{i} \nu}$ with Laurent expansion $\left(\gamma_{-r} \varpi_{i}^{-r}+\ldots+\gamma_{0} \varpi_{i}^{0}+H_{T}\right) \frac{d z_{i}}{z_{i} \nu}$. We also write $\hat{\Gamma}_{i}^{0}$ for the coefficient of $\varpi_{i}^{0} \frac{d z_{i}}{z_{i} \nu}$ in $\hat{\Gamma}_{i \nu}$.

We identify the tangent bundle of $\mathcal{A}_{i}$ with $\mathcal{A}_{i} \times \mathfrak{t}_{i}^{-r_{i}} / \mathfrak{t}_{i}^{1}$ using the pairing $\langle,\rangle_{\frac{d z_{i}}{z_{i}}}$. The differential of the map $\hat{\Gamma}_{i}: \widehat{\mathcal{M}} \rightarrow \mathcal{A}_{i}$ determines a section $d \hat{\Gamma}_{i}$ of $\Omega_{\widehat{\mathcal{M}}}^{1}\left(\mathfrak{t}_{i}^{-r_{i}} / \mathfrak{t}_{i}^{1}\right) \subset$ $\Omega_{\widehat{\mathcal{M}}}^{1}\left(\mathfrak{P}_{i}^{-r_{i}} / \mathfrak{P}_{i}^{1}\right)$.

Let $\psi_{i}: \widehat{\mathcal{M}} \rightarrow U_{i} \backslash \mathrm{GL}_{n}(\mathbb{C})$ be the composition of the projections $\widehat{\mathcal{M}} \rightarrow \widetilde{\mathcal{M}}_{i}$ and $\widetilde{\mathcal{M}}_{i} \rightarrow U_{i} \backslash \mathrm{GL}_{n}(\mathbb{C})$. Using the standard identification of $T\left(U_{i} \backslash \mathrm{GL}_{n}(\mathbb{C})\right)$ with $\left(\mathfrak{u}_{i} \backslash \mathfrak{g l}_{n}(\mathbb{C})\right) \times_{U_{i}} \mathrm{GL}_{n}(\mathbb{C})$, we define a map $\bar{\epsilon}_{i}$ from $\mathfrak{g l}_{n}(\mathbb{C})$ to global sections of $T\left(U_{i} \backslash \mathrm{GL}_{n}(\mathbb{C})\right)$ via $\bar{\epsilon}_{i}(X)\left(U_{i} g\right)=(\operatorname{Ad}(g) X, g)$. The pullback $\epsilon_{i}=\psi_{i}^{*} \bar{\epsilon}_{i}$ gives a map from $\mathfrak{g l}_{n}(\mathbb{C})$ to sections of $\psi_{i}^{*}\left(T\left(U_{i} \backslash \mathrm{GL}_{n}(\mathbb{C})\right)\right)$.

Identify $\widetilde{\mathcal{M}}_{i}$ with $\mathfrak{V}_{j} \times_{U_{j}} \mathrm{GL}_{n}(\mathbb{C})$ as in (3.1). Throughout this section, (3.1), we will use $m_{j}=\left(v_{j}, g_{j}\right) \in \mathfrak{V}_{j} \times_{U_{j}} \mathrm{GL}_{n}(\mathbb{C})$ to denote a point in $\widetilde{\mathcal{M}}_{i}$ and $m=\left(m_{j}\right)_{j \in I}$ to denote a point in $\widehat{\mathcal{M}} \subset \prod_{i \in I} \widetilde{\mathcal{M}}_{i}$.

We are now ready to define a collection of differential forms on $\widehat{\mathcal{M}}$.

(1) Define an endomorphism $\delta_{\mathrm{e}}{ }^{\prime}: \mathfrak{t}_{i}^{-r_{i}} / \mathfrak{t}_{i}^{1} \rightarrow \mathfrak{t}_{i}^{-r_{i}} / \mathfrak{t}_{i}^{1}$ by

$$
\delta_{\mathrm{e}}{ }^{\prime}\left(t_{-j} \varpi_{i}^{-j}\right)= \begin{cases}\frac{-e}{j} t_{-j} \varpi_{i}^{-j} & \text { if } j>0 ; \\ 0 & \text { if } j=0,\end{cases}
$$

and set $A_{\widetilde{\mathcal{M}}, i}=\delta_{\mathrm{e}}{ }^{\prime}\left(d \hat{\Gamma}_{i}\right)$. If $\nu=\frac{d z_{i}}{z_{i}}$, the first part of Lemma 4.1 implies that

$$
\tau_{\nu} A_{\widetilde{\mathcal{M}}, i}+\operatorname{ad}\left(\hat{\Gamma}_{i \nu}\right)\left(A_{\widetilde{\mathcal{M}}, i}\right)=d \hat{\Gamma}_{i \nu}-d \hat{\Gamma}_{i}^{0}+\mathfrak{t}_{i}^{1} .
$$

(2) Recall that $\left(\mathfrak{P}_{i} / \mathfrak{P}_{i}^{r_{i}+1}\right)_{\text {reg }}^{\vee} \cong \mathcal{A}_{i} \times_{T^{1}} P_{i}^{1} / P_{i}^{r+1}$. Given $m_{i} \in \widetilde{\mathcal{M}}_{i}$, we may write $\pi_{\mathfrak{P}_{i}}\left(v_{i}\right)=\left(a_{i}, p_{i}\right) \in \mathcal{A}\left(P_{i}, r_{i}\right) \times_{T^{1}} P_{i}^{1} / P_{i}^{r_{i}+1}$. Thus, $p_{i}$ is characterized by $\operatorname{Ad}\left(p_{i}\right)\left(\pi_{\mathfrak{P}_{i}}\left(v_{i}\right)\right)=a$. Define a bundle $B_{i}=\mathfrak{P}_{i}^{-r} / \mathfrak{P}_{i}^{1} \times_{U_{i}} \operatorname{GL}_{n}(\mathbb{C})$ over $U_{i} \backslash \mathrm{GL}_{n}(\mathbb{C})$, where $U_{i}$ acts on $\mathrm{GL}_{n}(\mathbb{C})$ by left multiplication and on $\mathfrak{P}_{i}^{-r} / \mathfrak{P}_{i}^{1}$ by Ad. We define $\Phi_{i} \in \Omega_{\widehat{\mathcal{M}}}^{1}\left(\psi_{i}^{*}\left(B_{i}\right)\right)$ by

$$
\Phi_{i}=\operatorname{Ad}\left(p_{i}^{-1}\right)\left(A_{\widetilde{\mathcal{M}}, i}\right),
$$

Note that $\Phi_{i}$ is well defined: $A_{\widetilde{\mathcal{M}}, i}$ is stabilized by $T^{1}$ and, since $u_{i}\left(a_{i}, p_{i}\right)=$ $\left(a_{i}, p_{i} u_{i}^{-1}\right)$,

$$
\operatorname{Ad}\left(u_{i}\right) \Phi_{i}\left(u_{i} m\right)=\Phi_{i}(m)
$$

(3) We define a third form $\Upsilon^{0}$ on $\widehat{\mathcal{M}}$ which has coefficients in $H^{0}\left(\mathbb{P}^{1} ; \mathfrak{g l}_{n \mathbb{P}^{1}}^{\mathbf{r}_{\mathbf{e}}}\right)$. The form

$$
\bar{\Phi}_{i}=\operatorname{Ad}\left(g_{i}^{-1}\right) \Phi_{i}+\Omega_{\widehat{\mathcal{M}}}^{1}\left(\mathfrak{g}_{i}\right) \in \Omega_{\widehat{\mathcal{M}}}^{1}\left(\left(\mathfrak{P}_{i}^{-r_{i}}\right)^{g_{i}} / \mathfrak{g}_{i}\right)
$$

is well defined on $\widehat{\mathcal{M}}$, so the product $\bar{\Phi}=\left(\bar{\Phi}_{i}\right)_{i \in I}$ determines a form in $\Omega_{\widehat{\mathcal{M}}}^{1}\left(\prod_{i \in I}\left(\left(\mathfrak{P}_{i}^{-r_{i}}\right)^{g_{i}} / \mathfrak{g}_{i}\right)\right)$. Using Definition 4.8, $\bar{\Phi}$ determines a form $\Upsilon^{0} \in$ $\Omega_{\widehat{\mathcal{M}}}^{1}\left(H^{0}\left(\mathbb{P}^{1} ; \mathfrak{g l}_{n \mathbb{P}^{1}}^{\mathbf{r}_{\mathbf{e}}}\right)\right)$. 
(4) The next form, $\Theta_{i}$, is defined on $\widehat{\mathcal{M}}$ with coefficients in $\psi_{i}^{*}\left(T\left(U_{i} \backslash \mathrm{GL}_{n}(\mathbb{C})\right)\right)$. Here, we identify $T\left(U_{i} \backslash \mathrm{GL}_{n}(\mathbb{C})\right)$ with $\mathfrak{u}_{i} \backslash \mathfrak{g l}_{n}(\mathbb{C}) \times_{U_{i}} \mathrm{GL}_{n}(\mathbb{C})$ in the usual way. Define a section of $\Omega_{\widehat{\mathcal{M}}}^{1}\left(\psi_{i}^{*} T\left(U_{i} \backslash \mathrm{GL}_{n}(\mathbb{C})\right)\right)$ by

$$
\Theta_{i}(m)=\left(d g_{i}\right) g_{i}^{-1}+\bar{\phi}_{i}\left(\Phi_{i}\right)-\bar{\phi}_{i}\left(\operatorname{Ad}\left(g_{i}\right)\left(\Upsilon^{0}\right)\right)+\Omega_{\widehat{\mathcal{M}}}^{1}\left(\mathfrak{u}_{i}\right) .
$$

The form $\Theta_{i}$ is well defined. By construction, $\bar{\phi}_{i}\left(\Phi_{i}\right)$ is well defined on $\widehat{\mathcal{M}}$. Furthermore, $\operatorname{Ad}\left(u_{i}\right) \bar{\phi}_{i}\left(\operatorname{Ad}\left(g_{i}\right) \Upsilon^{0}\right)\left(u_{i} m\right)=\bar{\phi}_{i}\left(\operatorname{Ad}\left(g_{i}\right) \Upsilon^{0}\right)(m)$ and

$$
d\left(u_{i} g_{i}\right) g_{i}^{-1} u_{i}^{-1} \in \operatorname{Ad}\left(u_{i}\right)\left(\left(d g_{i}\right) g_{i}^{-1}\right)+\Omega_{\widehat{\mathcal{M}}}^{1}\left(\mathfrak{u}_{i}\right) .
$$

(5) By Theorem 3.15, each point of $\widehat{\mathcal{M}}$ determines a unique form $\alpha \nu \in H^{0}\left(\mathbb{P}^{1} \backslash \mathbf{x} ; \Omega_{\mathbb{P}^{1}}^{1}\left(\mathfrak{g l}_{n \mathbb{P}^{1}}\right)\right)$. In particular, $d \alpha$ is a one form with coefficients in $H^{0}\left(\mathbb{P}^{1} \backslash \mathbf{x} ; \mathfrak{g l}_{n \mathbb{P}^{1}}\right)$. Note that $d \alpha$ depends on the choice of global form $\nu$.

(6) We define the last collection of forms on $\widehat{\mathcal{M}}$ to have coefficients in $\left(\mathfrak{g}_{i}^{-r_{i}^{\prime}} \frac{d z_{i}}{z_{i} \nu}\right) /\left(\mathfrak{g}_{i}^{1} \frac{d z_{i}}{z_{i} \nu}\right)$, where $\mathbf{r}_{\mathbf{e}}=\left(r_{i}^{\prime}\right)_{i \in I}$ as in Definition 4.7. Since $\mathrm{GL}_{n}(\mathbb{C})$ acts on $\widehat{\mathcal{M}}$, we have an isomorphism $\widehat{\mathcal{M}} \cong \mathrm{GL}_{n}(\mathbb{C}) \times_{\mathrm{GL}_{n}(\mathbb{C})} \widehat{\mathcal{M}}$. Let $\Xi_{i}^{\prime}$ be defined on $\mathrm{GL}_{n}(\mathbb{C}) \times \widehat{\mathcal{M}}$ by

$$
\Xi_{i}^{\prime}\left(g,\left(v_{j}, g_{j}\right)_{j \in I}\right)=\tau_{\nu} \Upsilon^{0}-d \alpha-\left[\Upsilon^{0}+g^{-1} d g, \alpha\right]+\Omega_{\mathrm{GL}_{n}(\mathbb{C}) \times \widehat{\mathcal{M}}}^{1}\left(\mathfrak{g}_{i}^{1} \frac{d z_{i}}{z_{i} \nu}\right) .
$$

The form $\Xi_{i}^{\prime}$ is $G$-invariant, hence induces a form $\Xi_{i}$ on $\widehat{\mathcal{M}}$. This follows from the observation

$$
d\left(\operatorname{Ad}\left(g^{-1}\right) \alpha\right)=\operatorname{Ad}\left(g^{-1}\right) d \alpha-\left[g^{-1} d g, \operatorname{Ad}\left(g^{-1}\right) \alpha\right] .
$$

Definition 5.1. Let $\mathcal{I}_{\mathrm{GL}_{n}}$ be the differential ideal on $\widehat{\mathcal{M}}(\mathbf{x}, \mathbf{P}, \mathbf{r})$ generated by the coefficients of

(1) $\Xi_{i} \in \Omega_{\widehat{\mathcal{M}}}^{1}\left(\left(\mathfrak{g}_{i}^{-r_{i}^{\prime}} \frac{d z_{i}}{z_{i} \nu}\right) /\left(\mathfrak{g}_{i}^{1} \frac{d z_{i}}{z_{i} \nu}\right)\right)$ and

(2) $\Theta_{i}-\epsilon\left(g^{-1} d g\right) \in \Omega_{\widehat{\mathcal{M}}}^{1}\left(\psi_{i}^{*}\left(\left(\mathfrak{u}_{i} \backslash \mathfrak{g l}_{n}(\mathbb{C})\right) \times_{U_{i}} \mathrm{GL}_{n}(\mathbb{C})\right)\right)$

for all $i \in I$.

Theorem 5.2. The ideal $\mathcal{I}_{\mathrm{GL}_{n}}$ satisfies Frobenius' integrability condition: $d \mathcal{I}_{\mathrm{GL}_{n}} \subset$ $\mathcal{I}_{\mathrm{GL}_{n}}$. Therefore, $\mathcal{I}_{\mathrm{GL}_{n}}$ determines a foliation of $\widehat{\mathcal{M}}(\mathbf{x}, \mathbf{P}, \mathbf{r})$. Moreover, a map $\sigma$ : $\Delta \rightarrow \widehat{\mathcal{M}}$ corresponds to a framed integrable deformation if and only if $\sigma^{*} \mathcal{I}_{\mathrm{GL}_{N}}=0$.

5.2. Proof of Integrability. In this section, we will prove Theorem 5.2, Throughout, we fix local sections $\hat{g}_{i}$ of the trivial $\mathrm{GL}_{n}\left(\mathfrak{o}_{i}\right)$-bundle over $\widehat{\mathcal{M}}$ such that $\hat{g}_{i} g_{i}^{-1} \in$ $P_{i}^{1}$ and $\hat{g}_{i} \cdot \alpha=\hat{\Gamma}_{i \nu}$. Observe that $\left(d \hat{g}_{i}\right) \hat{g}_{i}^{-1} \in\left(d g_{i}\right) g_{i}^{-1}+\mathfrak{P}_{i}^{1}$. We choose a lift $A_{\widetilde{\mathcal{M}}, i}^{\prime} \in \mathfrak{t}_{i}^{-r}$ of $A_{\widetilde{\mathcal{M}}, i}$ which satisfies the property

$$
\tau_{\nu} A_{\widetilde{\mathcal{M}}, i}^{\prime}+\left[\hat{\Gamma}_{i \nu}, A_{\widetilde{\mathcal{M}}, i}^{\prime}\right]=d \hat{\Gamma}_{i \nu}-d \hat{\Gamma}_{i}^{0} .
$$

If $B$ is a bundle over $\widehat{\mathcal{M}}$ and $\sigma \in \Omega_{\widehat{\mathcal{M}}}^{*}(B)$, we will use the shorthand " $\sigma \in \mathcal{I}_{\mathrm{GL}_{n}}$ " to mean $\sigma \in \mathcal{I}_{\mathrm{GL}_{n}} \otimes_{\mathcal{O}_{\widehat{\mathcal{M}}}} B$. If $B^{\prime}$ is another bundle and $f: B \rightarrow B^{\prime}$ is a bundle map, then $\sigma \in \mathcal{I}_{\mathrm{GL}_{n}}$ implies that $f(\sigma) \in \mathcal{I}_{\mathrm{GL}_{n}}$. In particular, this is the case if the bundles are trivial with $f$ induced by a $\mathbb{C}$-linear map on their fibers.

Lemma 5.3. Suppose that $X \in \Omega_{\widehat{\mathcal{M}}}^{1}\left(\mathfrak{P}_{i}^{1}\right)$ satisfies

$$
\tau_{\nu}(X)-\left[X, \hat{\Gamma}_{i \nu}\right] \in \mathcal{I}_{\mathrm{GL}_{n}} .
$$

Then, $X \in \mathcal{I}_{\mathrm{GL}_{n}}$. 
Proof. Without loss of generality, assume that $\nu=\frac{d z_{i}}{z_{i}}$ and $\tau_{\nu}=z_{i} \partial_{z_{i}}$. To simplify notation, we will suppress the subscript $i$ in the proof. Let $\psi: \Omega_{\widehat{\mathcal{M}}}^{1}\left(\mathfrak{P}^{1}\right) \rightarrow$ $\Omega_{\widehat{\mathcal{M}}}^{1}\left(\mathfrak{P}^{-r+1}\right)$ be defined by $\psi(Y)=\tau_{\nu}(Y)-\left[Y, \hat{\Gamma}_{\nu}\right]$.

First, we prove the case $r=0$, so $e=1$ and $P=\operatorname{GL}_{n}(\mathfrak{o})$. Note that $\hat{\Gamma}_{\nu} \in\left(\mathfrak{t}^{\mathfrak{b}}\right)^{\prime}$ by Definition 3.3 and $\tau_{\nu}(Y)=\ell Y$ for $Y \in \Omega_{\widehat{M}}^{1}\left(z^{\ell} \mathfrak{g l}_{n}(\mathbb{C})\right)$. It follows that $\psi$ : $z^{\ell} \mathfrak{g l}_{n}(\mathbb{C}) \rightarrow z^{\ell} \mathfrak{g l}_{n}(\mathbb{C})$ is an isomorphism, since $-\ell$ is never an eigenvalue of $\operatorname{ad}\left(\hat{\Gamma}_{\nu}\right)$. Now, suppose that $X \in \Omega_{\widehat{\mathcal{M}}}^{1}\left(\mathfrak{g}^{\ell}\right)+\mathcal{I}_{\mathrm{GL}_{n}}$ for $\ell \geq 1$, say $X \in X_{\ell}+\Omega_{\widehat{\mathcal{M}}^{1}}^{1}\left(\mathfrak{g}^{\ell+1}\right)+\mathcal{I}_{\mathrm{GL}_{n}}$ for some $X_{\ell} \in \Omega_{\widehat{\mathcal{M}}}^{1}\left(z^{\ell} \mathfrak{g l}_{n}(\mathbb{C})\right)$. (This holds trivially for $\ell=1$.) We see that the image of $\psi\left(X_{\ell}\right) \in \Omega_{\widehat{M}}^{1}\left(\mathfrak{g}^{\ell+1}\right)+\mathcal{I}_{\mathrm{GL}_{n}}$. However, we have shown that $\psi$ induces an automorphism of $\Omega_{\widehat{\mathcal{M}}}^{1}\left(\mathfrak{g}^{\ell} / \mathfrak{g}^{\ell+1}\right)$, so $X_{\ell} \in \Omega_{\widehat{\mathcal{M}}}^{1}\left(\mathfrak{g}^{\ell+1}\right)+\mathcal{I}_{\mathrm{GL}_{n}}$. By induction, we conclude that $X \in \mathcal{I}_{\mathrm{GL}_{n}}$.

Let $W_{\ell}=\operatorname{ker}\left(\bar{\pi}_{\mathfrak{t}}\right) \subset \mathfrak{P}^{\ell} / \mathfrak{P}^{\ell+1}$. We define the projection $\mathrm{p}_{\mathfrak{t}}: \mathfrak{g l}_{n}(F) \rightarrow \operatorname{ker}\left(\pi_{\mathfrak{t}}\right)$ by $\mathrm{p}_{\mathfrak{t}}(X)=X-\pi_{\mathfrak{t}}(X)$, and write $\overline{\mathrm{p}}_{\mathfrak{t}}: \mathfrak{P}^{\ell} / \mathfrak{P}^{\ell+1} \rightarrow W_{\ell}$ for the induced map on cosets. Let $\psi: \Omega_{\widehat{\mathcal{M}}}^{1}\left(\mathfrak{P}^{1}\right) \rightarrow \Omega_{\widehat{\mathcal{M}}}^{1}\left(\mathfrak{P}^{-r+1}\right)$ be defined by $\psi(Y)=\tau_{\nu}(Y)-\left[Y, \hat{\Gamma}_{\nu}\right]$. Since Lemma 4.1 states that $\psi(t)=\delta_{e}(t) \in \mathfrak{t}$ for all $t \in \Omega_{\widehat{\mathcal{M}}}^{1}\left(\mathfrak{t}^{1}\right), \mathrm{p}_{\mathfrak{t}} \circ \psi(t)=0$. This implies that $\mathrm{p}_{\mathfrak{t}}(\psi(Y))=\mathrm{p}_{\mathfrak{t}}\left(\psi\left(\mathrm{p}_{\mathfrak{t}}(Y)\right)\right)$ for all $Y \in \Omega_{\widehat{\mathcal{M}}}^{1}\left(\mathfrak{P}^{\ell}\right)$.

First, we show inductively that $X^{\prime}=\mathrm{p}_{\mathfrak{t}}(X) \in \mathcal{I}_{\mathrm{GL}_{n}}$. Suppose that $X^{\prime} \in \mathcal{I}_{\mathrm{GL}_{n}}+$ $\Omega_{\widehat{\mathcal{M}}}^{1}\left(\mathfrak{P}^{\ell}\right)$, say $X^{\prime} \in X_{\ell}^{\prime}+\mathcal{I}_{\mathrm{GL}_{n}}$ with $X_{\ell}^{\prime} \in \Omega_{\widehat{\mathcal{M}}}^{1}\left(\mathfrak{P}^{\ell}\right)$. Since $\psi(X) \in \mathcal{I}_{\mathrm{GL}_{n}}$, we obtain $\mathrm{p}_{\mathfrak{t}}\left(\psi\left(X_{\ell}^{\prime}\right)\right)=\mathrm{p}_{\mathfrak{t}}(\psi(X))+\mathcal{I}_{\mathrm{GL}_{n}} \subset \mathcal{I}_{\mathrm{GL}_{n}}$. By part (5) of Proposition 2.4 the map $\bar{\psi}: W_{\ell} \rightarrow W_{\ell-r}$ induced by $\mathrm{p}_{\mathfrak{t}} \circ \psi$ is an isomorphism, so $X_{\ell}^{\prime} \in \mathcal{I}_{\mathrm{GL}_{n}}+\Omega_{\widehat{\mathcal{M}}^{1}}^{1}\left(\mathfrak{P}^{\ell+1}\right)$. It follows that $X^{\prime} \in \mathcal{I}_{\mathrm{GL}_{n}}+\Omega_{\widehat{\mathcal{M}}}^{1}\left(\mathfrak{P}^{\ell+1}\right)$. Since the inductive hypothesis holds trivially for $\ell=1$, we conclude that $X^{\prime} \in \mathcal{I}_{\mathrm{GL}_{n}}$.

We now have $X \in \pi_{\mathfrak{t}}(X)+\mathcal{I}_{\mathrm{GL}_{n}}$ and also $\delta_{e}\left(\pi_{\mathfrak{t}}(X)\right)=\psi\left(\pi_{\mathfrak{t}}(X)\right)=\psi(X)-$ $\psi\left(X^{\prime}\right) \in \mathcal{I}_{\mathrm{GL}_{n}}$. Since $\delta_{e}$ is an automorphism of $\mathfrak{t}^{1}$, this gives $\pi_{\mathfrak{t}}(X) \in \mathcal{I}_{\mathrm{GL}_{n}}$, and hence $X \in \mathcal{I}_{\mathrm{GL}_{n}}$.

Lemma 5.4. Let $\mathcal{I}_{\mathrm{GL}_{n}}$ be the differential ideal defined above.

(1) The following identity holds modulo $\mathcal{I}_{\mathrm{GL}_{n}}$ :

$$
\Upsilon^{0}+g^{-1} d g \in \operatorname{Ad}\left(\hat{g}_{i}^{-1}\right) A_{\widetilde{\mathcal{M}}, i}^{\prime}+\hat{g}_{i}^{-1} d \hat{g}_{i}+\mathcal{I}_{\mathrm{GL}_{n}} .
$$

(2) Let $p_{i}=\hat{g}_{i} g_{i}^{-1}$. The following weaker identity holds modulo the ideal $\mathcal{I}_{\Theta}$ generated only by $\Theta_{i}-\epsilon\left(g^{-1} d g\right)$ :

$$
\operatorname{Ad}\left(g_{i}\right)\left(\Upsilon^{0}+g^{-1} d g\right) \in \operatorname{Ad}\left(p_{i}^{-1}\right) A_{\widetilde{\mathcal{M}}, i}^{\prime}+\left(d g_{i}\right) g_{i}^{-1}+\Omega_{\widehat{\mathcal{M}}}^{1}\left(\mathfrak{P}_{i}^{1}\right)+\mathcal{I}_{\Theta}
$$

(3) Finally, $d \hat{\Gamma}_{i}^{0} \in \mathcal{I}_{\mathrm{GL}_{n}}$.

Proof. Without loss of generality, we set $\nu=\frac{d z_{i}}{z_{i}}$ and $\tau_{\nu}=z_{i} \partial_{z_{i}}$. First, we note that $\Upsilon^{0}-\operatorname{Ad}\left(\hat{g}_{i}^{-1}\right)\left(A_{\widetilde{\mathcal{M}}, i}^{\prime}\right) \in \Omega_{\widehat{\mathcal{M}}}^{1}\left(\mathfrak{g}_{i}\right)$ by construction. Therefore,

$$
\begin{aligned}
& \operatorname{Ad}\left(g_{i}\right)\left(\Upsilon^{0}\right)+\operatorname{Ad}\left(g_{i}\right)\left(g^{-1} d g\right)-\left(\operatorname{Ad}\left(p_{i}^{-1}\right)\left(A_{\widetilde{\mathcal{M}}, i}^{\prime}\right)+\left(d g_{i}\right) g_{i}^{-1}\right) \in \\
&-\phi_{i}\left(\left(d g_{i}\right) g_{i}^{-1}+\operatorname{Ad}\left(p_{i}^{-1}\right)\left(A_{\widetilde{\mathcal{M}}, i}^{\prime}\right)-\operatorname{Ad}\left(g_{i}\right)\left(\Upsilon^{0}\right)\right)+\epsilon\left(g^{-1} d g\right)+\Omega_{\widehat{\mathcal{M}}}^{1}\left(\mathfrak{P}_{i}^{1}\right)= \\
&-\left(\Theta_{i}-\epsilon\left(g^{-1} d g\right)\right) \in \mathcal{I}_{\Theta} .
\end{aligned}
$$

This is the second part of the lemma. 
Since

$$
\tau_{\nu} \Upsilon^{0}-d \alpha-\left[\Upsilon^{0}+g^{-1} d g, \alpha\right]+\Omega_{\widehat{\mathcal{M}}}^{1}\left(\mathfrak{g}_{j}^{1} \frac{d z_{j}}{z_{j} \nu}\right)=\Xi_{j} \in \mathcal{I}_{\mathrm{GL}_{n}}
$$

we obtain

$$
\nu \wedge\left(\tau_{\nu} \Upsilon^{0}-d \alpha-\left[\Upsilon^{0}+g^{-1} d g, \alpha\right]\right) \in \mathcal{I}_{\mathrm{GL}_{n}} .
$$

This follows from the observation that the principal part at each $x_{j} \in \mathbb{P}^{1}$ lies in $\mathcal{I}_{\mathrm{GL}_{n}}$.

Let $\Upsilon^{\prime}=\operatorname{Ad}\left(\hat{g}_{i}\right)\left(\Upsilon^{0}+g^{-1} d g\right)-\left(d \hat{g}_{i}\right) \hat{g}_{i}^{-1}$. Using part (2), we see that $\Upsilon^{\prime}-$ $A_{\widetilde{\mathcal{M}}, i}^{\prime} \in \Omega_{\widehat{\mathcal{M}}}^{1}\left(\mathfrak{P}_{i}^{1}\right)+\mathcal{I}_{\mathrm{GL}_{n}}$. Substituting $\operatorname{Ad}\left(\hat{g}_{i}^{-1}\right)\left(\Upsilon^{\prime}\right)+\hat{g}_{i}^{-1} d \hat{g}_{i}=\Upsilon^{0}+g^{-1} d g$ and $\operatorname{Ad}\left(\hat{g}_{i}^{-1}\right) \hat{\Gamma}_{i \nu}+\hat{g}_{i}^{-1} \tau_{\nu}\left(\hat{g}_{i}\right)=\alpha$ into (5.4), we obtain

$$
\tau_{\nu}\left(\Upsilon^{\prime}\right)-d \hat{\Gamma}_{i \nu}-\left[\Upsilon^{\prime}, \hat{\Gamma}_{i \nu}\right] \in \mathcal{I}_{\mathrm{GL}_{n}}
$$

Since $d \Gamma_{i \nu}-d \hat{\Gamma}_{i}^{0}=\tau_{\nu}\left(A_{\widetilde{\mathcal{M}}, i}^{\prime}\right)-\left[A_{\widetilde{\mathcal{M}}, i}^{\prime}, \Gamma_{i \nu}\right]$, we deduce that

$$
\tau_{\nu}\left(\Upsilon^{\prime}-A_{\widetilde{\mathcal{M}}, i}^{\prime}\right)-\left[\Upsilon^{\prime}-A_{\widetilde{\mathcal{M}}, i}^{\prime}, \Gamma_{i \nu}\right] \in d \hat{\Gamma}_{i}^{0}+\mathcal{I}_{\mathrm{GL}_{n}}
$$

On the left hand side, $\tau_{\nu}\left(\Upsilon^{\prime}-A_{\widetilde{\mathcal{M}}, i}^{\prime}\right)$ lies in $\Omega_{\widehat{\mathcal{M}}}^{1}\left(\mathfrak{P}_{i}^{1}\right)+\mathcal{I}_{\mathrm{GL}_{n}}$. Therefore, $d \hat{\Gamma}_{i}^{0} \in$ $-\left[\Upsilon^{\prime}-A_{\widetilde{\mathcal{M}}, i}^{\prime}, \Gamma_{i \nu}\right]+\Omega_{\widehat{\mathcal{M}}}^{1}\left(\mathfrak{P}_{i}^{1}\right)+\mathcal{I}_{\mathrm{GL}_{n}}$. With $\mathrm{p}_{\mathfrak{t}_{i}}$ as in the proof of the previous lemma, we obtain

$$
\mathrm{p}_{\mathfrak{t}_{i}}\left(\left[\Upsilon^{\prime}-A_{\widetilde{\mathcal{M}}, i}^{\prime}, \Gamma_{i \nu}\right]\right) \in \mathcal{I}_{\mathrm{GL}_{n}}+\Omega_{\widehat{\mathcal{M}}}^{1}\left(\mathfrak{P}_{i}^{1}\right) .
$$

Suppose $\Upsilon^{\prime}-A_{\widetilde{\mathcal{M}}, i}^{\prime} \in \Omega_{\widehat{\mathcal{M}}}^{1}\left(\mathfrak{t}_{i}+\mathfrak{P}_{i}^{\ell}\right)+\mathcal{I}_{\mathrm{GL}_{n}}$ for $\ell \leq r$. By part (5) of Proposition 2.4 (5.5) implies that $\mathrm{p}_{\mathfrak{t}_{i}}\left(\Upsilon^{\prime}-A_{\widetilde{\mathcal{M}}, i}^{\prime}\right) \in \Omega_{\widetilde{\mathcal{M}}}^{1}\left(\mathfrak{P}_{i}^{\ell+1}\right)+\mathcal{I}_{\mathrm{GL}_{n}}$. We conclude that $\Upsilon^{\prime}-A_{\widetilde{\mathcal{M}}, i}^{\prime} \in$ $\Omega_{\widehat{\mathcal{M}}}^{1}\left(\mathfrak{P}_{i}^{r+1}+\mathfrak{t}_{i}\right)+\mathcal{I}_{\mathrm{GL}_{n}}$.

Finally, part (3) of Proposition 2.4 implies that $\left[\Upsilon^{\prime}-A_{\widetilde{\mathcal{M}}, i}, \Gamma_{i \nu}\right] \in \Omega_{\widehat{\mathcal{M}}}^{1}\left(\mathfrak{P}_{i}^{1}\right)+$ $\mathcal{I}_{\mathrm{GL}_{n}}$. Since $\mathfrak{t}^{b} \cap \mathfrak{P}^{1}=\{0\}, d \hat{\Gamma}_{i}^{0} \in \mathcal{I}_{\mathrm{GL}_{n}}$, giving part (3). The first statement now follows, since by Lemma 5.3. $\Upsilon^{\prime}-A_{\widetilde{\mathcal{M}}, i}^{\prime} \in \mathcal{I}_{\mathrm{GL}_{n}}$.

Lemma 5.5. The following holds modulo $\mathcal{I}_{\mathrm{GL}_{n}}$ :

$$
d\left(\Upsilon^{0}+g^{-1} d g\right)+\left(\Upsilon^{0}+g^{-1} d g\right) \wedge\left(\Upsilon^{0}+g^{-1} d g\right) \in \mathcal{I}_{\mathrm{GL}_{n}}
$$

Proof. Recall that $\Upsilon^{0}$ is defined as $\varsigma\left(\left(\bar{\Phi}_{i}\right)_{i \in I}\right)$. It follows that $d \Upsilon^{0}=\varsigma\left(\left(d \bar{\Phi}_{i}\right)_{i \in I}\right)$.

First, we will show that

$$
d\left(\Upsilon^{0}\right) \in-\left(\Upsilon^{0}+g^{-1} d g\right) \wedge\left(\Upsilon^{0}+g^{-1} d g\right)+\Omega_{\widehat{\mathcal{M}}}^{2}\left(\mathfrak{g}_{i}\right)+\mathcal{I}_{\mathrm{GL}_{n}}
$$

By construction, $\Upsilon^{0} \in \operatorname{Ad}\left(\hat{g}_{i}^{-1}\left(A_{\widetilde{\mathcal{M}}, i}^{\prime}\right)\right)+\Omega_{\widetilde{\mathcal{M}}}^{1}\left(\mathfrak{g}_{i}\right)$. Therefore,

$$
d\left(\Upsilon^{0}\right) \in-\left[\operatorname{Ad}\left(\hat{g}_{i}^{-1}\right)\left(A_{\widetilde{\mathcal{M}}, i}^{\prime}\right), \hat{g}_{i}^{-1} d \hat{g}_{i}\right]+\Omega_{\widehat{\mathcal{M}}}^{2}\left(\mathfrak{g}_{i}\right) .
$$

On the other hand, by Lemma 5.4 .

$$
\begin{aligned}
& \left(\Upsilon^{0}+g^{-1} d g\right) \wedge\left(\Upsilon^{0}+g^{-1} d g\right) \in \\
& \quad\left(\operatorname{Ad}\left(\hat{g}_{i}^{-1}\right) A_{\widetilde{\mathcal{M}}, i}^{\prime}+\hat{g}_{i}^{-1} d \hat{g}_{i}\right) \wedge\left(\operatorname{Ad}\left(\hat{g}_{i}^{-1}\right) A_{\widetilde{\mathcal{M}}, i}^{\prime}+\hat{g}_{i}^{-1} d \hat{g}_{i}\right)+\Omega_{\widetilde{\mathcal{M}}}^{2}\left(\mathfrak{g}_{i}\right)+\mathcal{I}_{\mathrm{GL}_{n}} .
\end{aligned}
$$

The right hand side is equivalent to $\left[\operatorname{Ad}\left(\hat{g}_{i}^{-1}\right) A_{\widetilde{\mathcal{M}}, i}^{\prime}, \hat{g}_{i}^{-1} d \hat{g}_{i}\right]+\Omega_{\widehat{\mathcal{M}}}^{2}\left(\mathfrak{g}_{i}\right)+\mathcal{I}_{\mathrm{GL}_{n}}$. This proves (5.6). 
It suffices to show that

$\left(\Upsilon^{0}+g^{-1} d g\right) \wedge\left(\Upsilon^{0}+g^{-1} d g\right) \in \varsigma\left(\left(\left(\Upsilon^{0}+g^{-1} d g\right) \wedge\left(\Upsilon^{0}+g^{-1} d g\right)+\Omega_{\widehat{\mathcal{M}}}^{2}\left(\mathfrak{g}_{i}\right)\right)_{i \in I}\right)-d g^{-1} d g$,

since the right hand side is equal to $-d\left(\Upsilon^{0}+g^{-1} d g\right)\left(\bmod \mathcal{I}_{\mathrm{GL}_{n}}\right)$ by the arguments above. By Lemma 4.10, $\left.\left(\Upsilon^{0}+g^{-1} d g\right)\right|_{z_{0}=0}=g^{-1} d g$. Moreover,

$$
\left.\left(\left(\Upsilon^{0}+g^{-1} d g\right) \wedge\left(\Upsilon^{0}+g^{-1} d g\right)\right)\right|_{z_{0}=0}=\left(g^{-1} d g\right) \wedge\left(g^{-1} d g\right)=-d g^{-1} d g .
$$

Another application of Lemma 4.10 gives (5.8).

Lemma 5.6. The following holds modulo $\mathcal{I}_{\mathrm{GL}_{n}}$ :

$$
d\left(\Theta_{i}-\epsilon\left(g^{-1} d g\right)\right) \in \Omega_{\widehat{\mathcal{M}}}^{2}\left(\mathfrak{u}_{i}\right)+\mathcal{I}_{\mathrm{GL}_{n}} .
$$

Proof. Throughout, we will write $\Upsilon=\Upsilon^{0}+g^{-1} d g$. As in Lemma 5.4, we write $p_{i}=\hat{g}_{i} g_{i}^{-1}$. By the same Lemma, we see that

$$
\operatorname{Ad}\left(g_{i}\right)(\Upsilon) \in \operatorname{Ad}\left(p_{i}^{-1}\right)\left(A_{\widetilde{\mathcal{M}}, i}^{\prime}\right)+p_{i}^{-1} d p_{i}+\left(d g_{i}\right) g_{i}^{-1}+\mathcal{I}_{\mathrm{GL}_{n}} .
$$

A sequence of calculations using the expression above and Lemma 5.5 produces

$$
\begin{aligned}
d\left(\operatorname{Ad}\left(g_{i}\right) \Upsilon\right) & =\left[\left(d g_{i}\right) g_{i}^{-1}, \operatorname{Ad}\left(g_{i}\right)(\Upsilon)\right]+\operatorname{Ad}\left(g_{i}\right) d(\Upsilon) \\
& \in\left[\left(d g_{i}\right) g_{i}^{-1}, \operatorname{Ad}\left(g_{i}\right)(\Upsilon)\right]-\operatorname{Ad}\left(g_{i}\right)(\Upsilon \wedge \Upsilon)+\mathcal{I}_{\mathrm{GL}_{n}} \\
& =\left[\left(d g_{i}\right) g_{i}^{-1}, \operatorname{Ad}\left(p_{i}^{-1}\right)\left(A_{\widetilde{\mathcal{M}}_{i}}^{\prime}\right)+p_{i}^{-1} d p_{i}+\left(d g_{i}\right) g_{i}^{-1}\right] \\
& -\left(\operatorname{Ad}\left(p_{i}^{-1}\right)\left(A_{\widetilde{\mathcal{M}}, i}^{\prime}\right)+p_{i}^{-1} d p_{i}+\left(d g_{i}\right) g_{i}^{-1}\right) \wedge\left(\operatorname{Ad}\left(p_{i}^{-1}\right)\left(A_{\widetilde{\mathcal{M}}_{, i}}^{\prime}\right)+p_{i}^{-1} d p_{i}+\left(d g_{i}\right) g_{i}^{-1}\right)+\mathcal{I}_{\mathrm{GL}_{n}} \\
& \left.=-d g_{i} d g_{i}^{-1}+d p_{i}^{-1} d p_{i}-\left[p_{i}^{-1} d p_{i}, \operatorname{Ad}\left(p_{i}^{-1}\right) A_{\widetilde{\mathcal{M}}_{, i}}^{\prime}\right)\right]+\mathcal{I}_{\mathrm{GL}_{n}} \\
& \left.=-d g_{i} d g_{i}^{-1}+d p_{i}^{-1} d p_{i}+d\left(\operatorname{Ad}\left(p_{i}^{-1}\right) A_{\widetilde{\mathcal{M}}, i}^{\prime}\right)\right)+\mathcal{I}_{\mathrm{GL}_{n}} .
\end{aligned}
$$

The last line is equivalent to $d \Phi_{i}-\left(d g_{i}\right) d g_{i}^{-1}+\mathcal{I}_{\mathrm{GL}_{n}}\left(\bmod \Omega_{\widehat{\mathcal{M}}^{1}}^{1}\left(\mathfrak{P}_{i}^{1}\right)\right)$. Therefore,

$$
d\left(\Phi_{i}+d g_{i} g_{i}^{-1}-\operatorname{Ad}\left(g_{i}\right)\left(\Upsilon^{0}-g^{-1} d g\right)\right) \in \Omega_{\widehat{\mathcal{M}}}^{1}\left(\mathfrak{P}_{i}^{1}\right)+\mathcal{I}_{\mathrm{GL}_{n}} .
$$

Applying $\bar{\phi}_{i}$ to the equation above gives $d\left(\Theta_{i}-\epsilon\left(g^{-1} d g\right)\right) \in \mathcal{I}_{\mathrm{GL}_{n}}$.

Proof of Theorem 5.2. As above, write $\Upsilon=\Upsilon^{0}+g^{-1} d g$. We have already shown that $d\left(\Theta_{i}-\epsilon\left(g^{-1} d g\right)\right) \in \mathcal{I}_{\mathrm{GL}_{n}}$. A calculation shows that

$$
d \Xi_{i}=\tau_{\nu}(d \Upsilon+\Upsilon \wedge \Upsilon)-[d \Upsilon+\Upsilon \wedge \Upsilon, \alpha]+\left[\Xi_{i}, \Upsilon\right]+\Omega_{\widehat{\mathcal{M}}}^{1}\left(\mathfrak{g}_{i}^{1} \frac{d z_{i}}{z_{i} \nu}\right)
$$

so by Lemma 5.5, $d \Xi_{i} \in \mathcal{I}_{\mathrm{GL}_{n}}$.

Finally, by Theorem 3.15, we may identify trivialized framed global deformations $(\mathbf{g}, \bar{V}, \tilde{\nabla})$ on $\mathbb{P}^{1} \times \Delta$ with analytic maps $\sigma: \Delta \rightarrow \widehat{\mathcal{M}}(\mathbf{x}, \mathbf{P}, \mathbf{r})$. Note that $\sigma^{*} \mathcal{I}_{\mathrm{GL}_{n}}=$ $\{0\}$ if and only if $\sigma^{*}\left(\Theta_{i}-\epsilon\left(g^{-1} d g\right)\right)=0$ and $\sigma^{*} \Xi_{i}=0$ for all $i$, and the vanishing of these forms is equivalent to conditions (11) and (2) of Corollary 4.13 Also, by Lemma 5.5, $\sigma^{*} \mathcal{I}_{\mathrm{GL}_{n}}=\{0\}$ implies $\sigma^{*}(d \Upsilon+\Upsilon \wedge \Upsilon)=0$, which is equivalent to condition (3).

Theorem 5.2 and Lemma 5.5 immediately show that the third conditions in Theorem 4.12 and Corollary 4.13 are redundant. 
Theorem 5.7. Let $(\mathbf{g}, \bar{V}, \tilde{\nabla})$ be a good framed deformation. The following statements are equivalent.

(1) $(\mathbf{g}, \bar{V}, \tilde{\nabla})$ is integrable.

(2) $(\mathrm{g}, \bar{V}, \tilde{\nabla})$ is $\mathrm{GL}_{n}(R)$-gauge-equivalent to a deformation satisfying the first two conditions of Theorem 4.12.

(3) There exists $g \in \mathrm{GL}_{n}(R)$ such that the first two conditions of Corollary 4.13 are satisfied.

\section{EXAMPLE}

In this section, we give an explicit example of the system of equations constructed above. We will consider a space of rank $n$ meromorphic connections on $\mathbb{P}^{1}$ with $m$ singularities of slope $\frac{1}{n}$. Let $\mathrm{x}$ be a set of $m$ finite points, and set $P_{i}=I_{i}$ and $r_{i}=1$ for all $i$. If $z$ is the usual coordinate on $\mathbb{P}^{1}$, we write $z_{i}=\left(z-\xi_{i}\right)$. Accordingly, $\varpi_{i}=\varpi_{T_{i}}=N+z_{i} E \in \mathfrak{P}_{i}^{1}$, where $N$ is the regular nilpotent matrix in Jordan form and $E$ is the elementary matrix $E_{n 1}$. Note that $\varpi_{i}^{-1}=N^{\prime}+\frac{1}{z_{i}} E^{\prime}$, where $N^{\prime}$ and $E^{\prime}$ are the transposes of $N$ and $E$ respectively.

We choose our one form to be $\nu=d z$, so $\tau_{\nu}=\partial_{z}=\frac{d}{d z}$. Choose a point $\left(U_{i} g_{i}, \alpha_{i}\right)_{i \in I} \in \widehat{\mathcal{M}}$ corresponding to a connection $\nabla$, and write $[\nabla]=\alpha \nu$. Thus, $\alpha \in \alpha_{i \nu}+\mathfrak{I}_{i}^{1}$ at each $i$ and $\sum_{i \in I} \operatorname{Res}_{i}(\alpha \nu)=0$. To simplify calculations, we assume that the normalized formal type of $\nabla$ at $\xi_{i}$ has the representative $\frac{1}{z_{i}}\left(-\frac{a_{i}}{n} \varpi_{i}^{-1}+H_{T}\right)$ under the pairing $\langle,\rangle_{\nu}$.

We write $A_{\widetilde{\mathcal{M}}, i}=\varpi_{i}^{-1} d a_{i}$ and $\operatorname{Ad}\left(g_{i}\right)(\alpha)=\frac{1}{z_{i}}\left(-\frac{a_{i}}{n} \varpi_{i}^{-1}-\frac{1}{n}\left(D_{i}+X_{i}\right)+H_{T}+\mathfrak{g}_{i}\right)$ for some traceless diagonal matrix $D_{i}$ and $X_{i} \in \mathfrak{u}_{i}$. By definition,

$$
\Phi_{i}=\left(\varpi_{i}^{-1} d a_{i}+D_{i} \frac{d a_{i}}{a_{i}}\right)+\Omega_{\Delta}^{1}\left(\Im_{i}^{1}\right) .
$$

Therefore,

$$
\Upsilon^{0}=\sum_{i \in I} \operatorname{Ad}\left(g_{i}^{-1}\right)\left(z_{i}^{-1} E^{\prime} d a_{i}\right)
$$

and

$$
\phi_{i}\left(\Upsilon^{0}\right)=\sum_{j \in I \backslash\{i\}} \frac{1}{\xi_{i}-\xi_{j}} \operatorname{Ad}\left(g_{j}^{-1}\right)\left(E^{\prime} d a_{j}\right) .
$$

We conclude that equation (11) from Theorem 4.12 is equivalent to

$$
\begin{aligned}
& \left(d g_{i} g_{i}^{-1}, g_{i}\right)= \\
& \quad\left(\left[\sum_{j \in I \backslash i} \frac{1}{\xi_{i}-\xi_{j}} \operatorname{Ad}\left(g_{i} g_{j}^{-1}\right)\left(E^{\prime} d a_{j}\right)\right]-\left[N^{\prime} d a_{i}+D_{i} \frac{d a_{i}}{a_{i}}\right]+\mathfrak{u}_{i}, g_{i}\right)
\end{aligned}
$$

in $\left(\mathfrak{g l}_{n}(\mathbb{C}) / \mathfrak{u}_{i}\right) \times_{U_{i}} \mathrm{GL}_{n}(\mathbb{C})$.

Now, we consider equation (2) of Theorem 4.12 At $\xi_{i}$, applying $\operatorname{Ad}\left(g_{i}\right)$ to the principal part of the curvature gives us

$$
\begin{aligned}
& \quad-z_{i}^{-2} E^{\prime} d a_{i} \in \\
& -\frac{1}{n z_{i}}\left(\varpi_{i}^{-1} d a_{i}+d D_{i}+d X_{i}\right)+\left[\operatorname{Ad}\left(g_{i}\right)\left(\Upsilon^{0}\right)-\left(d g_{i}\right) g_{i}^{-1}, \operatorname{Ad}\left(g_{i}\right)(\alpha)\right]+\Omega_{\Delta}^{1}\left(\mathfrak{g}_{i}\right) .
\end{aligned}
$$


First, we calculate $\left[z_{i}^{-1} E^{\prime}, \operatorname{Ad}\left(g_{i}\right)(\alpha)\right]+\mathfrak{g}_{i}$. Observe that $\left[z_{i}^{-1} E^{\prime}, \operatorname{Ad}\left(g_{i}\right)(\alpha) d z\right]$ is a one form on $\mathbb{P}_{\Delta}^{1}$ with poles along $\mathbf{x}$. Thus,

$$
\left[z_{i}^{-1} E^{\prime}, \operatorname{Ad}\left(g_{i}\right)(\alpha) d z\right] \in\left(-\frac{1}{n z_{i}}\left[z_{i}^{-1} E^{\prime}, N^{\prime} a_{i}+D_{i}+X_{i}-n H_{T}\right]+\frac{1}{z_{i}} \rho\right) d z+\mathfrak{g}_{i} d z
$$

where $\rho$ is the residue term. By the residue theorem, $\rho+\sum_{j \in I} \operatorname{Res}_{j}\left(\left[z_{i}^{-1} E^{\prime}, \operatorname{Ad}\left(g_{i}\right)(\alpha) d z\right]\right)=$ 0 . We conclude that

$$
\begin{aligned}
\rho & =\frac{1}{n} \sum_{j \in I \backslash\{i\}} \operatorname{Res}_{j}\left(\left[z_{i}^{-1} E^{\prime}, \operatorname{Ad}\left(g_{i} g_{j}^{-1}\right)\left(\varpi_{i}^{-1} a_{j}+D_{j}+X_{j}-n H_{T}\right)\right] \frac{d z}{z_{j}}\right) \\
& =\frac{1}{n} \sum_{j \in I \backslash\{i\}} \frac{1}{\xi_{j}-\xi_{i}}\left[E^{\prime}, \operatorname{Ad}\left(g_{i} g_{j}^{-1}\right)\left(N^{\prime} a_{j}+D_{j}+X_{j}-n H_{T}\right)\right]-\frac{1}{\left(\xi_{j}-\xi_{i}\right)^{2}}\left[E^{\prime}, \operatorname{Ad}\left(g_{i} g_{j}^{-1}\right) E^{\prime} a_{j}\right] .
\end{aligned}
$$

By Proposition 4.2 we see that

$$
-z_{i}^{-2} E^{\prime} d a_{i}=-\frac{1}{n z_{i}}\left(\varpi_{i}^{-1} d a_{i}\right)+\frac{1}{z_{i}}\left[\varpi_{i}^{-1} d a_{i}, H_{T}\right] .
$$

Comparing (6.2), (6.3), and (6.4), we obtain the condition

$$
\begin{aligned}
& (6.5) \quad-\frac{1}{n z_{i}}\left(d\left(D_{i}+X_{i}\right)+\left[z_{i}^{-1} E^{\prime} d a_{i}, N^{\prime} a_{i}+D_{i}+X_{i}-n H_{T}\right]\right)+ \\
& \frac{1}{z_{i}} \rho d a_{i}+\left[\operatorname{Ad}\left(g_{i}\right)\left(\Upsilon^{0}\right)-z_{i}^{-1} E^{\prime} d a_{i}-\left(d g_{i}\right) g_{i}^{-1}, \operatorname{Ad}\left(g_{i}\right)(\alpha)\right]-\frac{1}{z_{i}}\left[\varpi_{i}^{-1} d a_{i}, H_{T}\right] \in \Omega_{\Delta}^{1}\left(\mathfrak{g}_{i}\right) .
\end{aligned}
$$

Now, by (6.1),

$$
\begin{aligned}
& \text { (6.6) } \quad\left[\operatorname{Ad}\left(g_{i}\right) \Upsilon^{0}-z_{i}^{-1} E^{\prime} d a_{i}-\left(d g_{i}\right) g_{i}^{-1}, z_{i}^{-1} E^{\prime} a_{i}\right] \in \\
& {\left[\phi_{i}\left(\operatorname{Ad}\left(g_{i}\right) \Upsilon^{0}\right)-\left(d g_{i}\right) g_{i}^{-1}, z_{i}^{-1} E^{\prime} a_{i}\right]-\sum_{j \in I \backslash\{i\}} \frac{1}{\left(\xi_{j}-\xi_{i}\right)^{2}}\left[\operatorname{Ad}\left(g_{i} g_{j}^{-1}\right)\left(E^{\prime}\right), E^{\prime}\right] a_{i} d a_{j}+\Omega_{\Delta}^{1}\left(\mathfrak{g}_{i}^{1}\right)} \\
& =\left[N^{\prime} d a_{i}+D_{i} \frac{d a_{i}}{a_{i}}, z_{i}^{-1} E^{\prime} a_{i}\right]-\sum_{j \in I \backslash\{i\}} \frac{1}{\left(\xi_{j}-\xi_{i}\right)^{2}}\left[\operatorname{Ad}\left(g_{i} g_{j}^{-1}\right)\left(E^{\prime}\right), E^{\prime}\right] a_{i} d a_{j}+\Omega_{\Delta}^{1}\left(\mathfrak{g}_{i}^{1}\right) .
\end{aligned}
$$

On the other hand,

$$
\begin{aligned}
& {\left[\operatorname{Ad}\left(g_{i}\right) \Upsilon^{0}-z_{i}^{-1} E^{\prime} d a_{i}, N^{\prime} a_{i}+D_{i}+X_{i}-n H_{T}\right] \in} \\
& {\left[\phi_{i}\left(\operatorname{Ad}\left(g_{i}\right) \Upsilon^{0}\right), N^{\prime} a_{i}+D_{i}+X_{i}-n H_{T}\right]+\Omega_{\Delta}^{1}\left(\mathfrak{g}_{i}^{1}\right)} \\
& \quad=\left[\sum_{j \in I \backslash\{i\}} \frac{1}{\xi_{i}-\xi_{j}} \operatorname{Ad}\left(g_{i} g_{j}^{-1}\right)\left(E^{\prime} d a_{j}\right), N^{\prime} a_{i}+D_{i}+X_{i}-n H_{T}\right]+\Omega_{\Delta}^{1}\left(\mathfrak{g}_{i}^{1}\right) .
\end{aligned}
$$

Finally,

$$
\left[z_{i}^{-1} E^{\prime} d a_{i}, H_{T}\right]-\left[\varpi_{i}^{-1} d a_{i}, H_{T}\right]=\left[-N^{\prime} d a_{i}, H_{T}\right]
$$


We substitute (6.6), (6.7), and (6.8) into 6.5):

$$
\begin{gathered}
d\left(D_{i}+X_{i}\right)-\left[\left(d g_{i}\right) g_{i}^{-1}, N^{\prime} a_{i}+D_{i}+X_{i}-n H_{T}\right] \equiv\left[-N^{\prime} d a_{i}, n H_{T}\right]+ \\
\sum_{j \in I \backslash\{i\}} \frac{1}{\xi_{j}-\xi_{i}}\left[E^{\prime} d a_{i}, \operatorname{Ad}\left(g_{i} g_{j}^{-1}\right)\left(N^{\prime} a_{j}+D_{j}+X_{j}-n H_{T}\right)\right]-\frac{1}{\left(\xi_{j}-\xi_{i}\right)^{2}}\left[E^{\prime}, \operatorname{Ad}\left(g_{i} g_{j}^{-1}\right) E^{\prime}\right] a_{j} d a_{i} \\
+\sum_{j \in I \backslash\{i\}} \frac{1}{\left(\xi_{j}-\xi_{i}\right)^{2}}\left[\operatorname{Ad}\left(g_{i} g_{j}^{-1}\right)\left(E^{\prime}\right), E^{\prime}\right] a_{i} d a_{j} \\
-\sum_{j \in I \backslash\{i\}} \frac{1}{\xi_{i}-\xi_{j}}\left[\operatorname{Ad}\left(g_{i} g_{j}^{-1}\right)\left(E^{\prime} d a_{j}\right), N^{\prime} a_{i}+D_{i}+X_{i}-n H_{T}\right] \quad\left(\bmod \Omega_{\Delta}^{1}\left(\mathfrak{g}_{i}^{1}\right)\right) .
\end{gathered}
$$

Equivalently, if we apply $\operatorname{Ad}\left(g_{i}^{-1}\right)$ and divide both sides by $-n$, we obtain

$$
\begin{aligned}
d \phi_{i}\left(z_{i} \alpha\right)=\sum_{j \in I \backslash\{i\}}\left(\frac{1}{\xi_{j}-\xi_{i}}(\right. & {\left.\left[\operatorname{Ad}\left(g_{j}^{-1}\right) E^{\prime}, \phi_{i}\left(z_{i} \alpha\right)\right] d a_{j}+\left[\operatorname{Ad}\left(g_{i}^{-1}\right) E^{\prime}, \phi_{j}\left(z_{j} \alpha\right)\right] d a_{i}\right) } \\
+ & \left.\frac{1}{n} \frac{1}{\left(\xi_{j}-\xi_{i}\right)^{2}}\left[\operatorname{Ad}\left(g_{i}^{-1}\right) E^{\prime}, \operatorname{Ad}\left(g_{j}^{-1}\right) E^{\prime}\right] d\left(a_{i} a_{j}\right)\right)
\end{aligned}
$$

since $\left[N^{\prime} d a_{i}, H_{T}\right]=\frac{1}{n} N^{\prime} d a_{i}$.

\section{APPENDIX}

In this section, we will show that $\mathcal{I}_{\mathrm{GL}_{n}}$ is a Pfaffian system in the sense of [7, II.2.4]. We will need to describe a (local) minimal basis for $\mathcal{I}_{\mathrm{GL}_{n}}$. Since $\mathcal{I}_{\mathrm{GL}_{n}}$ is independent of the choice of one-form $\nu$, we may assume that $\nu=\frac{d z_{i}}{z_{i}}$ when working locally at $x_{i}$. Moreover, the basis only requires a local description. Thus, over a sufficiently small neighborhood $W_{i} \subset U_{i} \backslash \mathrm{GL}_{n}(\mathbb{C})$, we may choose a smooth algebraic slice of coset representatives $\sigma_{i}: W_{i} \rightarrow \mathrm{GL}_{n}(\mathbb{C})$. For brevity, we will write $g_{i}^{\sigma}=\sigma_{i}\left(U_{i} g_{i}\right)$.

We define a map $\xi_{i}: \mathfrak{g}_{i} \rightarrow \Omega_{\psi_{i}^{-1}\left(W_{i}\right)}^{1}$ using the residue-trace pairing in (2.1):

$$
\xi_{i}(X)=\left\langle X, \operatorname{Ad}\left(g_{i}^{\sigma}\right) \Xi_{i}\right\rangle_{\nu}
$$

By part (2) of Proposition 2.4, the map $\pi_{\mathfrak{t}_{i}}$ induces a map $\pi_{\mathfrak{t}_{i}}^{\prime}: \mathfrak{P}_{i} / \mathfrak{P}_{i}^{r} \rightarrow \mathfrak{t}_{i} / \mathfrak{t}_{i}^{r}$. Choose vector space lifts $L_{i}^{\text {od }} \subset \mathfrak{P}$ of $\operatorname{ker}\left(\pi_{\mathfrak{t}_{i}}^{\prime}\right)$ and $L_{i}^{\mathfrak{u}} \subset \mathfrak{g}$ of $\mathfrak{g}_{i} / \mathfrak{P}_{i}$. Note that these vector spaces are 0 when $r=0$.

Let $W=\bigcap_{i \in I} \psi_{i}^{-1}\left(W_{i}\right) \subset \widehat{\mathcal{M}}(\mathbf{x}, \mathbf{P}, \mathbf{r})$. We locally define the 'off-diagonal' and $\mathfrak{u}$ components of $\Xi_{i}$ to be $\Xi_{i}^{o d}=\left.\xi_{i}\right|_{L^{o d}} \in \Omega_{W}^{1}\left(\left(L^{o d}\right)^{\vee}\right)$ and $\Xi_{i}^{\mathfrak{u}}=\left.\xi_{i}\right|_{L^{\mathfrak{u}}} \in \Omega_{W}^{1}\left(\left(L^{\mathfrak{u}}\right)^{\vee}\right)$ respectively.

Lemma 7.1. Let $\mathcal{J}$ be a differential ideal in $\Omega_{\widehat{\mathcal{M}}}^{*}$. Then $\xi_{i}\left(\mathfrak{g}_{i}\right) \subset \mathcal{J}$ if and only if $\Xi_{i} \in \mathcal{J}$.

Proof. By definition of $\Xi_{i}, \mathfrak{g}_{i}^{r_{i}^{\prime}+1} \subset \operatorname{ker}\left(\xi_{i}\right)$, so $\xi_{i}$ induces an element $\bar{\xi}_{i} \in \operatorname{Hom}_{\mathbb{C}}\left(\mathfrak{g}_{i} / \mathfrak{g}_{i}^{s}, \Omega_{W}^{1}\right) \cong$ $\Omega_{W}^{1}\left(\left(\mathfrak{g}_{i} / \mathfrak{g}_{i}^{r_{i}^{\prime}+1}\right)^{\vee}\right)$. Note that $\xi_{i}\left(\mathfrak{g}_{i}\right) \subset \mathcal{J}$ if and only $\bar{\xi}_{i} \in \mathcal{J}$. Moreover, $\bar{\xi}_{i}$ and $\left.\operatorname{Ad}\left(g_{i}^{\sigma}\right)\right)\left(\Xi_{i}\right)$ correspond under the isomorphism $\left.\left(\mathfrak{g}_{i} / \mathfrak{g}_{i}^{r_{i}^{\prime}+1}\right)^{\vee}\right) \cong \mathfrak{g}^{-r_{i}^{\prime}} / \mathfrak{g}_{i}^{1}$ given by the perfect pairing $\langle,\rangle_{\nu}$, so $\bar{\xi}_{i} \in \mathcal{J}$ if and only if $\left.\operatorname{Ad}\left(g_{i}^{\sigma}\right)\right)\left(\Xi_{i}\right) \in \mathcal{J}$. The result now follows, since this last fact holds if and only if $\Xi_{i} \in \mathcal{J}$. 
Lemma 7.2. Suppose that $\mathcal{J}$ is a differential ideal on $\widehat{\mathcal{M}}$ containing $d \hat{\Gamma}_{i}^{0}$. If

$$
\operatorname{Ad}\left(g_{i}\right)\left(\Upsilon^{0}\right)+\operatorname{Ad}\left(g_{i}\right)\left(g^{-1} d g\right)-\left(d g_{i}\right) g_{i}^{-1}+\Omega_{\widehat{\mathcal{M}}}^{1}\left(\mathfrak{P}_{i}^{1}\right) \in \Phi_{i}+\mathcal{J},
$$

then $\operatorname{Ad}\left(g_{i}\right)\left(\Xi_{i}\right) \in \Omega_{\widehat{\mathcal{M}}}^{1}\left(\left[\mathfrak{P}_{i}^{1}, \operatorname{Ad}\left(g_{i}\right)(\alpha)\right]+\mathfrak{P}_{i}^{1} \frac{d z_{i}}{z_{i}}\right)+\mathcal{J}$.

Proof. Without loss of generality, let $\nu=\frac{d z_{i}}{z_{i}}$. Recall that $\tau_{\nu}\left(A_{\widetilde{\mathcal{M}}_{i}}^{\prime}\right)-d \hat{\Gamma}_{i \nu}+d \hat{\Gamma}_{i}^{0}+$ $\left[\hat{\Gamma}_{i \nu}, A_{\widetilde{\mathcal{M}}, i}^{\prime}\right]=0$. Write $\alpha^{\prime}=\hat{g}_{i}^{-1} \cdot\left(\hat{\Gamma}_{i \nu}-\hat{\Gamma}_{i}^{0}\right)$, so $\alpha-\alpha^{\prime}=\operatorname{Ad}\left(\hat{g}_{i}^{-1}\right)\left(\hat{\Gamma}_{i}^{0}\right)$. Set $Z=\operatorname{Ad}\left(\hat{g}_{i}^{-1}\right) A_{\widetilde{\mathcal{M}}, i}^{\prime}+\hat{g}_{i}^{-1} d \hat{g}_{i}$.

By Proposition 4.2, $\tau_{\nu}(Z)-d \alpha^{\prime}+\left[\alpha^{\prime}, Z\right]=0$. It follows that

$$
\tau_{\nu}(Z)-d \alpha+[\alpha, Z]=-\operatorname{Ad}\left(\hat{g}_{i}^{-1}\right) d \hat{\Gamma}_{i}^{0} \in \mathcal{J} .
$$

By definition, $\operatorname{Ad}\left(\hat{g}_{i}^{-1}\right) A_{\widetilde{\mathcal{M}}, i}+\Omega_{\widehat{\mathcal{M}}}^{1}\left(\left(\mathfrak{P}_{i}^{1}\right)^{g_{i}}\right)=\operatorname{Ad}\left(g_{i}^{-1}\right) \Phi_{i}$ and $\hat{g}_{i}^{-1} d \hat{g}_{i} \in g_{i}^{-1} d g_{i}+$ $\Omega_{\widehat{\mathcal{M}}}^{1}\left(\left(\mathfrak{P}_{i}^{1}\right)^{g_{i}}\right)$. Therefore, subtracting the above expression from $\Xi_{i}$ and using the hypothesis yields

$$
\begin{aligned}
\Xi_{i} \in \tau_{\nu}\left(\Upsilon^{0}+g^{-1} d g-\operatorname{Ad}\left(\hat{g}_{i}^{-1}\right) A_{\widetilde{\mathcal{M}}, i}-\hat{g}_{i}^{-1} d \hat{g}_{i}\right)- \\
{\left[\Upsilon^{0}+g^{-1} d g-\operatorname{Ad}\left(\hat{g}_{i}^{-1}\right) A_{\widetilde{\mathcal{M}}, i}-\hat{g}_{i}^{-1} d \hat{g}_{i}, \alpha\right]+\Omega_{\widehat{\mathcal{M}}}^{1}\left(\left(\mathfrak{P}_{i}^{1}\right)^{g_{i}}\right)+\mathcal{J} . }
\end{aligned}
$$

The lemma follows after applying $\operatorname{Ad}\left(g_{i}\right)$ to both sides of the equation above.

Proposition 7.3. On the neighborhood $W$ defined above, $\mathcal{I}_{\mathrm{GL}_{n}}$ is generated by $\Theta_{i}-\epsilon\left(g^{-1} d g\right), \Xi_{i}^{o d}, \Xi_{i}^{\mathfrak{u}}$ and $d \hat{\Gamma}_{i}^{0}$ for $i \in I$.

Proof. As above, we assume that $\nu=\frac{d z_{i}}{z_{i}}$. By Lemma [7.1 it suffices to show that $\xi_{i}\left(\mathfrak{g}_{i}\right)$ lies in the ideal $\mathcal{I}^{\prime}$ generated by $d \hat{\Gamma}_{i}^{0}, \Theta_{i}-\epsilon\left(g^{-1} d g\right), \Xi_{i}^{o d}$, and $\Xi_{i}^{\mathfrak{u}}$, since $\mathcal{I}^{\prime} \subset \mathcal{I}_{\mathrm{GL}_{n}}$.

Let $\mathcal{I}_{1}$ be the ideal generated by $\left\{\Theta_{i}-\epsilon\left(g^{-1} d g\right)\right\}$ and $d \hat{\Gamma}_{i}^{0}$. By the second part of Lemma 5.4 $\Upsilon^{0}+g^{-1} d g \in \operatorname{Ad}\left(\hat{g}_{i}^{-1}\right) A_{\widetilde{\mathcal{M}}, i}+\hat{g}_{i}^{-1} d \hat{g}_{i}+\Omega_{W}^{1}\left(\left(\mathfrak{P}_{i}^{1}\right)^{g_{i}}\right)+\mathcal{I}_{1}$. Lemma 7.2 states that

$$
\operatorname{Ad}\left(g_{i}^{\sigma}\right) \Xi_{i} \in \Omega_{W}^{1}\left(\left[\mathfrak{P}_{i}^{1}, \operatorname{Ad}\left(g_{i}^{\sigma}\right)(\alpha)\right]+\mathfrak{P}_{i}^{1}\right)+\mathcal{I}_{1} \subset \Omega_{W}^{1}\left(\mathfrak{P}^{-r+1}\right)+\mathcal{I}_{1} .
$$

Therefore, $\xi_{i}\left(\mathfrak{P}_{i}^{r}\right) \subset \mathcal{I}_{1}$, and it follows that the ideal $\mathcal{I}_{2}$ generated by $\mathcal{I}_{1}$ and the $\Xi_{i}^{o d}$ 's is independent of the choice of lift for $L^{\text {od }}$.

We will now show that $\operatorname{Ad}\left(g_{i}^{\sigma}\right)\left(\Xi_{i}\right) \in \mathcal{I}_{2}+\Omega_{W}^{1}\left(\mathfrak{P}_{i}^{1}\right)$. By (17.2), there exists $X \in \Omega_{W}^{1}\left(\mathfrak{P}_{i}^{1}\right)$ such that $\operatorname{Ad}\left(g_{i}^{\sigma}\right) \Xi_{i} \in\left[X, \operatorname{Ad}\left(g_{i}^{\sigma}\right)(\alpha)\right]+\Omega_{W}^{1}\left(\mathfrak{P}_{i}^{1}\right)+\mathcal{I}_{2}$. Take $1 \leq j \leq r$, and assume inductively that there exists $X \in \Omega_{W}^{1}\left(\mathfrak{P}_{i}^{j}\right)$ such that

$$
\operatorname{Ad}\left(g_{i}^{\sigma}\right) \Xi_{i} \in\left[X, \operatorname{Ad}\left(g_{i}^{\sigma}\right)(\alpha)\right]+\mathcal{I}_{2}+\Omega_{W}^{1}\left(\mathfrak{P}_{i}^{1}\right) .
$$

Part (3) of Proposition 2.4 shows that $\left[X, \operatorname{Ad}\left(g_{i}^{\sigma}\right)(\alpha)\right]+\Omega_{W}^{1}\left(\mathfrak{P}_{i}^{j-r+1}\right) \in \operatorname{ker}\left(\bar{\pi}_{\mathfrak{t}_{i}}\right)$. Thus, by part (4) of the same proposition, $\left\langle\left[X, \operatorname{Ad}\left(g_{i}^{\sigma}\right)(\alpha)\right], \mathfrak{t}_{i}^{r-j}\right\rangle_{\nu}=\{0\}$. Since $\Xi_{i}^{o d} \in \mathcal{I}_{2},\left\langle\left[X, \operatorname{Ad}\left(g_{i}^{\sigma}\right)(\alpha)\right], Y\right\rangle_{\nu} \in \mathcal{I}_{2}$ for $Y \in \operatorname{ker}\left(\pi_{\mathfrak{t}_{i}}\right) \cap \mathfrak{P}^{r-j}$. Combining these two facts gives $\left\langle\left[X, \operatorname{Ad}\left(g_{i}^{\sigma}\right)(\alpha)\right], \mathfrak{P}^{r-j}\right\rangle_{\nu} \in \mathcal{I}_{2}$, and we conclude that $\left[X, \operatorname{Ad}\left(g_{i}^{\sigma}\right)(\alpha)\right] \in$ $\Omega_{W}^{1}\left(\mathfrak{P}_{i}^{j-r+1}\right)+\mathcal{I}_{2}$. Part (5) of Proposition 2.4 now implies that $X \in \pi_{\mathfrak{t}}(X)+$ $\Omega_{W}^{1}\left(\mathfrak{P}_{i}^{j+1}\right)+\mathcal{I}_{2}$. Finally, since there exists $p \in P_{i}^{1}$ such that $\operatorname{Ad}(p)\left(\operatorname{Ad}\left(g_{i}^{\sigma}\right)(\alpha)\right) \in$ $\mathfrak{t}_{i}^{-r}+\mathfrak{P}_{i}^{1}$, we see that $X-\operatorname{Ad}\left(p^{-1}\right)\left(\pi_{\mathfrak{t}}(X)\right) \in \Omega_{W}^{1}\left(\mathfrak{P}_{i}^{j+1}\right)+\mathcal{I}_{2}$ satisfies (7.3) for $j+1$. By induction, we obtain (7.3) for $r+1$. This gives $\operatorname{Ad}\left(g_{i}^{\sigma}\right)\left(\Xi_{i}\right) \in \mathcal{I}_{2}+\Omega_{W}^{1}\left(\mathfrak{P}_{i}^{1}\right)$ and hence $\xi_{i}\left(\mathfrak{P}_{i}\right) \subset \mathcal{I}_{2}$.

Finally, since $\mathfrak{g}_{i}=L_{\mathfrak{u}}+\mathfrak{P}_{i}$, we see that $\xi_{i}\left(\mathfrak{g}_{i}\right) \subset \mathcal{I}^{\prime}$ as desired. Note that it also follows that the ideal $\mathcal{I}^{\prime}$ is independent of the choice of $L_{\mathfrak{u}}$. 
In the following, let $\iota: \widehat{\mathcal{M}} \rightarrow \prod_{i \in I} \widetilde{\mathcal{M}}_{i}$ be the inclusion and $T^{*} \iota: \iota^{*}\left(\Omega_{\prod_{i \in I}}^{1} \widetilde{\mathcal{M}}_{i}\right) \rightarrow$ $\Omega_{\widehat{M}}^{1}$ the induced bundle map. The generators of $\mathcal{I}_{\mathrm{GL}_{n}}$ lift to a set of generators

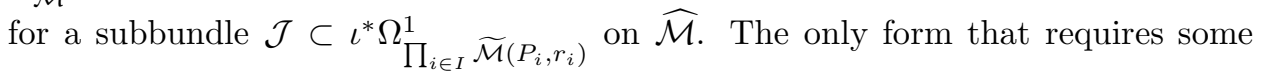
explanation is $\Xi_{i}$. Suppose that $m=\left(\left(U_{j} g_{j}, \alpha_{j}\right)_{j \in I}\right) \in \prod_{i \in I} \widetilde{\mathcal{M}}_{i}$. We write $\alpha_{i \nu}$ for a representative of $\alpha$ in $\left(\mathfrak{g}_{i}^{-r_{i}^{\prime}} \frac{d z_{i}}{z_{i} \nu}\right) /\left(\mathfrak{g}_{i}^{1} \frac{d z_{i}}{z_{i}}\right)$. Therefore, if $m \in \widehat{\mathcal{M}}, \alpha_{i \nu}=\alpha+\mathfrak{g}_{i}^{1} \frac{d z_{i}}{z_{i} \nu}$. We may rewrite $\Xi_{i}$ as

$$
\Xi_{i}=\tau_{\nu} \Upsilon^{0}-d \alpha_{i \nu}-\left[\Upsilon^{0}+g^{-1} d g, \alpha_{i \nu}\right] .
$$

It is clear that $T^{*} \iota\left(\Xi_{i}\right)$ is the usual $\Xi_{i}$.

Lemma 7.4. Let $\mu$ be the moment map for the action of $\mathrm{GL}_{n}(\mathbb{C})$ on $\prod_{i \in I} \widetilde{\mathcal{M}}\left(P_{i}, r_{i}\right)$. Then, $d \mu \subset \mathcal{J}$.

Proof. Let $\nu$ be the global choice of one form. By construction, $\mu\left(\left(U_{i} g_{i}, \alpha_{i}\right)_{i \in I}\right)=$ $\sum_{i \in I} \operatorname{res}\left(\alpha_{i}\right)$. However,

$$
\begin{aligned}
\sum_{i \in I} \operatorname{Res}_{i}\left(\nu \wedge \Xi_{i}\right) & =\sum_{i \in I} \operatorname{Res}_{i}\left(\nu \wedge \tau_{\nu}\left(\Upsilon^{0}\right)\right)-\operatorname{Res}_{i}\left(\nu \wedge d \alpha_{i \nu}\right)-\operatorname{Res}_{i}\left(\nu \wedge\left[\Upsilon^{0}+g^{-1} d g, \alpha\right]\right) \\
& =\sum_{i \in I} \operatorname{Res}_{i}\left(\nu \wedge d \alpha_{i \nu}\right) .
\end{aligned}
$$

Here, $\sum_{i \in I} \operatorname{Res}_{i}\left(\nu \wedge \tau_{\nu}\left(\Upsilon^{0}\right)\right)$ and $\sum_{i \in I} \operatorname{Res}_{i}\left(\nu \wedge\left[\Upsilon^{0}, \alpha\right]\right)$ vanish by the residue theorem. Since $\operatorname{Res}_{i}\left(\nu \wedge \alpha_{i \nu}\right)=\operatorname{res}_{i}\left(\alpha_{i}\right)$, we see that $d \mu=\sum_{i \in I} \operatorname{Res}_{i}\left(\nu \wedge d \alpha_{i \nu}\right)$ lies in $\mathcal{J}$.

Lemma 7.5. Let $\hat{\rho}: \mathrm{GL}_{n}(\mathbb{C}) \times_{\mathrm{GL}_{n}(\mathbb{C})} \widehat{\mathcal{M}} \rightarrow \widehat{\mathcal{M}}$ be the usual isomorphism. Define

$$
\bar{\Xi}_{i}=\tau_{\nu} \Upsilon^{0}-d \alpha-\left[\Upsilon^{0}, \alpha\right]+\Omega_{\widehat{\mathcal{M}}}^{1}\left(\mathfrak{g}_{i}^{1} \frac{d z_{i}}{z_{i} \nu}\right) .
$$

Then, $\hat{\rho}^{*} \Theta_{i}(h, m)=\Theta_{i}(m)-\epsilon\left(h^{-1} d h\right)$ and $\hat{\rho}^{*} \bar{\Xi}(h, m)=\operatorname{Ad}(h) \hat{\rho}^{*}(\Xi)(h, m)$.

Proof. Recalling the definition of $\Theta_{i}$ in Section 5, one easily checks that

$$
\begin{aligned}
\hat{\rho}^{*} \Theta_{i}(h, m)=d\left(g_{i} h^{-1}\right) h g_{i}^{-1}+\bar{\phi}\left(\Phi_{i}\right)-\bar{\phi}\left(\operatorname{Ad}\left(g_{i} g^{-1}\right)\left(\Upsilon^{0}\right)\right) & +\Omega_{\mathrm{GL}_{n}(\mathbb{C}) \times_{\mathrm{GL}_{n}(\mathbb{C})} \widehat{\mathcal{M}}^{\left(\mathfrak{u}_{i}\right)}} \\
& =\Theta_{i}(m)-\epsilon\left(h^{-1} d h\right) .
\end{aligned}
$$

Furthermore, $d\left(\hat{\rho}^{*}(\alpha)\right)(h, m)=d(\operatorname{Ad}(h)(\alpha))=\operatorname{Ad}(h)(\alpha)+\left[(d h) h^{-1}, \operatorname{Ad}(h)(\alpha)\right]$. Therefore,

$$
\begin{aligned}
& \operatorname{Ad}(h) \hat{\rho}^{*}\left(\Xi_{i}\right)(h, m)= \\
& \begin{aligned}
\tau_{\nu}\left(\operatorname{Ad}(h)\left(\Upsilon^{0}\right)\right)-\operatorname{Ad}(h) d \alpha-\left[\operatorname{Ad}(h)\left(\Upsilon^{0}+h^{-1} d h\right), \operatorname{Ad}(h)(\alpha)\right] & \left.+\Omega_{\widehat{\mathcal{M}}^{1}}^{1} \mathfrak{g}_{i}^{1} \frac{d z_{i}}{z_{i} \nu}\right) \\
& =\hat{\rho}^{*} \bar{\Xi}_{i}(h, m) .
\end{aligned}
\end{aligned}
$$

Proposition 7.6. The ideal $\mathcal{I}_{\mathrm{GL}_{n}}$ is a Pfaffian system. 
Proof. Fix a point $m=\left(U_{i} g_{i}, \alpha_{i}\right)_{i \in I} \in \widehat{\mathcal{M}}$, with corresponding formal types $\left(A_{i}\right)_{i \in I}$. We will first show that $\mathcal{J}$ has full rank when restricted to $\left(\prod_{i \in I} \widetilde{\mathcal{M}}\left(A_{i}\right)\right) \cap \widehat{\mathcal{M}}$. In other words, the restriction of $\mathcal{J}$ to $\prod_{i \in I} \widetilde{\mathcal{M}}\left(A_{i}\right)$ spans $\Omega_{\prod_{i \in I}^{1} \widetilde{\mathcal{M}}\left(A_{i}\right)}$.

Let $\psi_{i}^{\prime}: \widetilde{\mathcal{M}}\left(P_{i}, r_{i}\right) \rightarrow U_{i} \backslash \mathrm{GL}_{n}$ be the natural projection. Choose neighborhoods $W_{i} \subset U_{i} \backslash \mathrm{GL}_{n}$ of $\psi_{i}(m)$ as above, and let $\hat{W}=\prod_{i \in I}\left(\psi_{i}^{\prime}\right)^{-1}\left(W_{i}\right)$ be the corresponding neighborhood of of $m$ in $\prod_{i \in I} \widetilde{\mathcal{M}}\left(P_{i}, r_{i}\right)$. There are isomorphisms $\hat{W} \cong$ $\prod_{i}\left(\mathfrak{P}_{i} / \mathfrak{P}_{r+1}\right)_{r e g}^{\vee} \times W_{i}$ and $W(\mathbf{A})=\hat{W} \cap \prod_{i \in I} \widetilde{\mathcal{M}}\left(A_{i}\right) \cong \prod_{i \in I}\left(\pi_{\mathfrak{P}^{1}}\right)^{-1}\left(\mathcal{O}_{i}^{1}\right) \times W_{i}$.

By Lemma 7.5, $\mathcal{J}$ is generated by $\Theta_{i}$ and $\bar{\Xi}_{i}$. Restricting $\mathcal{J}$ to $W(\mathbf{A})$, we see that all terms involving $A_{\widetilde{\mathcal{M}}, i}$ vanish. In particular, $\Upsilon^{0}$ and $\Phi_{i}$ vanish, so $\Theta_{i}$ becomes $\left(d g_{i}\right) g_{i}^{-1}+\Omega_{W(\mathbf{A})}^{1}\left(\mathfrak{u}_{i}\right)$, and $\bar{\Xi}_{i}$ is simply $d \alpha+\Omega_{W(\mathbf{A})}^{1}\left(\mathfrak{g}_{i}^{1} \frac{d z_{i}}{z_{i} \nu}\right)$. Write $\operatorname{Ad}\left(g_{i}\right)(\alpha)=v \in$ $\left(\pi_{\mathfrak{P}^{1}}\right)^{-1}\left(\mathcal{O}_{i}^{1}\right)$. Since $d \alpha=\operatorname{Ad}\left(g_{i}^{-1}\right)\left(d v-\left[\left(d g_{i}\right) g_{i}^{-1}, v\right]\right)$, it is easily checked that the coefficients of $\left(d g_{i}\right) g_{i}^{-1}$ and $d \alpha$ span $T_{m}^{*}(W(\mathbf{A}))$. On the other hand, a calculation using [2, Lemma 3.17] shows that $\operatorname{dim} T_{m}^{*}(W(\mathbf{A}))=\operatorname{dim} \mathfrak{g l}_{n}(\mathbb{C}) / \mathfrak{u}+\operatorname{dim} \mathfrak{g} / \mathfrak{P}^{r}-$ $\operatorname{dim} \mathfrak{t}^{1} / \mathfrak{t}^{r}$, and so the coefficients of the set of generators for $\mathcal{J}$ in Proposition 7.3 give a basis for $T_{m}^{*}(W(\mathbf{A}))$. Thus, $\mathcal{J}$ is a $\operatorname{rank} \sum_{i \in I} \operatorname{dim}\left(\widetilde{\mathcal{M}}\left(A_{i}\right)\right)$ Pfaffian system.

Lemma 7.4 shows that $d \mu \in \mathcal{J}$. Since the coefficients of $d \mu$ span the conormal bundle of $\widehat{\mathcal{M}}$ in $\prod_{i \in I} \widetilde{\mathcal{M}}\left(P_{i}, r_{i}\right)$, we see that the image of $\mathcal{J}$ in $\Omega_{\widehat{\mathcal{M}}}^{1}$, i.e., $\mathcal{I}_{\mathrm{GL}_{n}}$, is a $\operatorname{rank} \sum_{i \in I} \operatorname{dim}\left(\widetilde{\mathcal{M}}\left(A_{i}\right)\right)-n^{2}$ Pfaffian system.

\section{REFERENCES}

[1] P. Boalch, "Symplectic manifolds and isomonodromic deformations," Adv. Math. 163 (2001), 137-205.

[2] C. Bremer and D. S. Sage, "Moduli Spaces of Irregular Singular Connections," arXiv:1004.4411 1 [math.AG], 2010.

[3] C. J. Bushnell, "Hereditary orders, Gauss sums, and supercuspidal representations of $\mathrm{GL}_{N}$," J. Reine Angew. Math. 375/376 (1987), 184-210.

[4] N. Chriss and V. Ginzburg, Representation theory and complex geometry, Birkhäuser, Boston, 1997.

[5] E. Frenkel, Langlands correspondence for loop groups, Cambridge University Press, New York, 2007.

[6] V. Guillemin and S. Sternberg, Geometric Asymptotics, Mathematical Surveys, No. 14, American Mathematical Society, Providence, 1977.

[7] G. Hector and U. Hirsch, Introduction to the Geometry of Foliations, Part A, Aspects of Mathematics, Friedr. Vieweg and Sohn, Braunschweig, 1981.

[8] M. Jimbo and T. Miwa, "Monodromy preserving deformations of linear differential equations with rational coefficients II," Physica D 2 (1981), 407-448.

[9] M. Jimbo, T. Miwa, and K. Ueno, "Monodromy preserving deformations of linear ordinary differential equations with rational coefficients I," Physica D 2 (1981), 306-352.

[10] B. Malgrange, Équations Différentielles à Coefficients Polynomiaux, Progress in Mathematics, Vol. 96, Birkhäuser Boston, Inc., Boston, MA, 1991.

[11] J. Marsden and T. Ratiu, "Reduction of Poisson Manifolds," Letters in Mathematical Physics, 11 (1986), 161-169.

[12] A. Moy and G. Prasad, "Unrefined minimal $K$-types for $p$-adic groups," Invent. Math. 116 (1994), 393-408.

[13] C. Sabbah, Isomonodromic Deformations and Frobenius Manifolds, Universitext, Springer, Berlin, 2007.

[14] L. Schlesinger, "Über eine Klasse von Differentialsystemen beliebiger Ordnung mit festen kritischen Punkten," J. Reine Angew. Math., 141 (1912), 96-145. 
Department of Mathematics, Louisiana State University, Baton Rouge, LA 70803

E-mail address: cbremer@math.lsu.edu

E-mail address: sage@math.1su.edu 


\title{
ISOMONODROMIC DEFORMATIONS OF CONNECTIONS WITH SINGULARITIES OF PARAHORIC FORMAL TYPE
}

\author{
CHRISTOPHER L. BREMER AND DANIEL S. SAGE
}

\begin{abstract}
In previous work, the authors have developed a geometric theory of fundamental strata to study connections on the projective line with irregular singularities of parahoric formal type. In this paper, the moduli space of connections that contain regular fundamental strata with fixed combinatorics at each singular point is constructed as a smooth Poisson reduction. The authors then explicitly compute the isomonodromy equations as an integrable system. This result generalizes work of Jimbo, Miwa, and Ueno to connections whose singularities have parahoric formal type.
\end{abstract}

\section{INTRODUCTION}

The study of transcendental solutions to differential equations has a long pedigree in mathematics. An important innovation in this field from the turn of the century was Schlesinger's observation that families of solutions to linear differential equations often satisfy interesting nonlinear equations. His work on monodromypreserving deformations of Fuchsian differential equations produced a remarkable family of nonlinear differential equations which satisfy the Painlevé property, namely, that the only movable singularities are simple poles $[15$.

For example, consider a family of regular singular differential equations on $\mathbb{P}^{1}$ of the form

$$
\frac{d}{d z} \Phi=\sum_{i=1}^{p} \frac{1}{z-x_{i}} A_{i} \Phi
$$

where $x_{1}, \ldots, x_{p} \in \mathbb{P}^{1}$ and $A_{i}$ is a matrix valued holomorphic function in the coordinates $x_{1}, \ldots, x_{p}$. A family of fundamental solutions to (1.1) has constant monodromy if and only if the $A_{i}$ 's satisfy the Schlesinger equations:

$$
d A_{i}=\sum_{j \neq i}\left[A_{i}, A_{j}\right] \frac{d\left(x_{i}-x_{j}\right)}{x_{i}-x_{j}} .
$$

(See [13, IV.1] for a contemporary exposition.) In general, we will refer to the differential equations satisfied by a family of linear differential equations with constant monodromy as isomonodromy equations.

It took almost seventy years for progress to be made on the isomonodromy problem for irregular singular differential equations. In 1981, Jimbo, Miwa, and Ueno characterized the isomonodromy equations for certain generic families of irregular

2010 Mathematics Subject Classification. Primary:14D24; Secondary: 34Mxx, 53D30.

Key words and phrases. meromorphic connections, irregular singularities, moduli spaces, Poisson reduction, fundamental stratum, isomonodromy.

The research of the second author was partially supported by NSF grant DMS-0606300 and NSA grant H98230-09-1-0059. 
singular differential equations 9 . One explanation for the long delay is that the monodromy map for irregular singular point differential equations is significantly more complicated; it involves the asymptotic behavior of solutions along Stokes sectors at each singular point, so it is less explicitly topological than the monodromy map in the regular singular case. The proof of Jimbo, Miwa and Ueno required a much clearer geometric picture of the monodromy map.

In this paper, we will think of linear differential equations in terms of meromorphic connections $\nabla$ on a trivial vector bundle $V$ on $\mathbb{P}^{1}$. After fixing a basis for $V$, we may write $\nabla=d+\alpha$, where $d$ is the usual exterior derivative and $\alpha$ is an $\operatorname{End}(V)$-valued meromorphic one-form. Roughly speaking, if one considers the moduli stack $\mathcal{M}_{\mathrm{DR}}$ of meromorphic connections on $\mathbb{P}^{1}$, there is a formal RiemannHilbert map to the moduli stack $\mathcal{M}_{\mathrm{B}}$ of irregular monodromy representations. 1$]$ An explicit description of the irregular Riemann-Hilbert correspondence may be found in $[10$.

If one can find a smooth family $\mathcal{M}^{\prime}$ that maps to $\mathcal{M}_{\mathrm{DR}}$, the Malgrange-Sibuya theorem [10] implies that the fibers of the monodromy map are a foliation of $\mathcal{M}^{\prime}$. In particular, the isomonodromy equations should correspond to an integrable distribution on $\mathcal{M}^{\prime}$. By an observation of Boalch [1, Appendix], $\mathcal{L} \subset \mathcal{M}^{\prime}$ is a leaf of this foliation if and only if the family of connections corresponding to $\mathcal{L}$ is integrable, i.e., there exists a connection $\bar{\nabla}$ on $\mathcal{L} \times \mathbb{P}^{1}$ with the property that $\left.\bar{\nabla}\right|_{\{x\} \times \mathbb{P}^{1}}$ is a representative of the isomorphism class $x \in \mathcal{M}_{\mathrm{DR}}$. Throughout the paper, we will suppress the monodromy point of view in favor of this integrability condition. As an example, we describe the smooth family of framed connections $\widetilde{\mathcal{M}}_{\text {JMU }}$ which was constructed by Boalch, building on work of Jimbo, Miwa and Ueno.

Fix a finite collection of points $\left(x_{i}\right)_{i \in I} \subset \mathbb{P}^{1}$ and a vector of non-negative integers $\left(r_{i}\right)_{i \in I} 2$ Points in $\widetilde{\mathcal{M}}_{\mathrm{JMU}}$ correspond to isomorphism classes of connections $\nabla=$ $d+\alpha$, singular at $x_{i}$, with the following additional data: there is a collection of framings $g_{i} \in \mathrm{GL}_{n}(\mathbb{C})$ such that the Laurent expansion of $\operatorname{Ad}\left(g_{i}\right) \alpha$ at $x_{i}$ has the form

$$
\operatorname{Ad}\left(g_{i}\right) \alpha=\left(A_{r_{i}} \frac{1}{\left(z-x_{i}\right)^{r_{i}}}+\ldots+A_{1} \frac{1}{z-x_{i}}+A_{0}\right) \frac{d z}{z-x_{i}},
$$

where $A_{i} \in \mathfrak{g l}_{n}(\mathbb{C})$ and the leading term $A_{r_{i}}$ is a regular diagonal matrix. The moduli space $\widetilde{\mathcal{M}}_{\mathrm{JMU}}$ is, in fact, smooth. The isomonodromy equations may be expressed in terms of a Pfaffian system involving terms $\Theta_{i}$, which control the dynamics of the framings, and terms $\Xi_{i}$, which are essentially the principal parts of the curvature of $\bar{\nabla}[9]$.

In 2, the authors describe smooth moduli spaces of framed connections with arbitrary slope, generalizing a construction of 11. The goal of this paper is to study isomonodromic deformations of such connections. The primary technical tool is a local invariant of meromorphic connections called the fundamental stratum, which plays the role of the leading term. A stratum is a triple $(P, r, \beta)$, consisting of a parahoric subgroup $P \subset \mathrm{GL}_{n}(\mathbb{C}[[z]])$, a non-negative integer $r$, and a 'nondegenerate' linear functional on the $r^{t h}$ graded piece associated to the canonical filtration on the Lie algebra of $P$. The relevant condition on connections which

\footnotetext{
${ }^{1}$ Here, DR stands for the "DeRham" theory of meromorphic connections, and B stands for the "Betti" theory of irregular monodromy representations.

${ }^{2}$ There is a slight simplification here: Jimbo, Miwa, and Ueno and Boalch allow $x_{i}$ to vary in $\mathbb{P}^{1}$.
} 
assures smoothness of the moduli space is that $\nabla$ must contain a 'regular' stratum. This approach is described in detail in Section 2 .

The primary motivation behind the introduction of fundamental strata into the study of connections comes from the geometric Langlands program, which in this case suggests an analogy between wildly ramified adelic representations of $\mathrm{GL}_{n}$ and irregular monodromy representations of rank $n$ on $\mathbb{P}^{1}$ (see [5] or, for a more physical interpretation, [16). The fundamental stratum (alternatively, the minimal $K$-type) was originally used as a tool for classifying wildly ramified representations of a reductive group over a $p$-adic field $[3,12$. Thus, one hopes that a dictionary between fundamental strata and families of monodromy representations will better illuminate the wild ramification case of the geometric Langlands correspondence.

We conclude with a short overview of the results in this paper. Suppose that $\mathbf{x}=\left(x_{i}\right)_{i \in I} \subset \mathbb{P}^{1}$ is a collection of singular points, $\mathbf{P}=\left(P_{i}\right)_{i \in I}$ is a collection of uniform parahoric subgroups $P_{i} \subset \mathrm{GL}_{n}\left(\mathbb{C}\left[\left[z-x_{i}\right]\right]\right)$, and $\mathbf{r}=\left(r_{i}\right)_{i \in I}$ is a vector of non-negative integers. In Section 3, we give a construction of the moduli space $\widetilde{\mathcal{M}}(\mathbf{x}, \mathbf{P}, \mathbf{r})$ consisting of isomorphism classes of connections with compatible framings on $\mathbb{P}^{1}$ that contain regular strata of the form $\left(P_{i}, r_{i}, \beta_{i}\right)$ at $x_{i}$. By Proposition 3.15 and Theorem 3.16, $\widetilde{\mathcal{M}}(\mathbf{x}, \mathbf{P}, \mathbf{r})$ is a Poisson manifold; moreover, the symplectic leaves correspond to connected components of moduli spaces of connections with a fixed formal isomorphism class at each singular point. When all of the parahoric subgroups are maximal, i.e., $P_{i}=\mathrm{GL}_{n}\left(\mathbb{C}\left[\left[z-x_{i}\right]\right]\right), \widetilde{\mathcal{M}}(\mathbf{x}, \mathbf{P}, \mathbf{r})=\widetilde{\mathcal{M}}_{\mathrm{JMU}}$.

In Section 4, we calculate the isomonodromy equations for connections corresponding to points of $\widetilde{\mathcal{M}}(\mathbf{x}, \mathbf{P}, \mathbf{r})$ (Theorem 4.12). In this context, the framing data is given by a coset $U \backslash \mathrm{GL}_{n}(\mathbb{C})$, where $U \subset \mathrm{GL}_{n}(\mathbb{C})$ is the unipotent subgroup determined by $P$. In particular, part of the isomonodromy data is a time dependent flow on an affine bundle over a partial flag manifold. Since $U$ is trivial when $P$ is a maximal parahoric subgroup, this phenomenon is absent in the isomonodromy equations of Jimbo, Miwa and Ueno.

In Section [5, we describe a differential ideal $\overline{\mathcal{I}}$ on the moduli space $\widetilde{\mathcal{M}}(\mathbf{x}, \mathbf{P}, \mathbf{r})$ corresponding to the isomonodromy equations. To be precise, we construct a differential ideal $\mathcal{I}$ on a principal $\mathrm{GL}_{n}(\mathbb{C})$-bundle over $\widetilde{\mathcal{M}}(\mathbf{x}, \mathbf{P}, \mathbf{r})$ and then show that it descends. The main result of the paper, Theorem 5.1 states that both $\overline{\mathcal{I}}$ and $\mathcal{I}$ are integrable Pfaffian systems. Moreover, there is a natural correspondence between the leaves of the foliation determined by $\overline{\mathcal{I}}$ and isomonodromic deformations of framed connections. The proof is deferred to Section 6 .

Finally, in Section 7 we compute an explicit example of the isomonodromy equations in the case where $P_{i}$ is an Iwahori subgroup and $r_{i}=1$ for all $i$. To the authors' knowledge, this is a completely new example of an integrable system on a Poisson manifold.

\section{Formal Types}

In this section, we review some results from the geometric theory of fundamental strata and recall how they may be used to associate formal types to irregular singular connections. Let $F=k((z))$ be field of formal Laurent series with coefficients in a field $k$ in characteristic zero, and let $\mathfrak{o} \subset F$ be the corresponding power series ring. Let $\hat{V}$ be an $n$-dimensional $F$ vector space. A lattice chain $\mathcal{L}=\left\{L^{i}\right\}_{i \in \mathbb{Z}}$ is a collection of $\mathfrak{o}$-lattices in $\hat{V}$ satisfying the following properties: $L^{i} \supset L^{i+1}$, and 
$z L^{i}=L^{i+e}$ with a fixed period $e>0$. We say $\mathcal{L}$ is uniform if $\operatorname{dim}_{k} L^{i} / L^{i+1}=n / e$ for all $i$; the lattice chain is complete if $e=n$.

Definition 2.1. A uniform parahoric subgroup $P \subset \operatorname{GL}(\hat{V})$ is the stabilizer of a uniform lattice chain $\mathcal{L}$, i.e., $P=\left\{g \in \operatorname{GL}(\hat{V}) \mid g L^{i}=L^{i}\right.$ for all $\left.i\right\}$. The Lie algebra of $P$ is the parahoric subalgebra $\mathfrak{P} \subset \mathfrak{g l}(\hat{V})$ consisting of $\mathfrak{P}=\left\{p \in \mathfrak{g l}(\hat{V}) \mid p L^{i} \subset\right.$ $L^{i}$ for all $\left.i\right\}$. Note that $\mathfrak{P}$ is in fact an associative subalgebra of $\mathfrak{g l}(\hat{V})$. An Iwahori subgroup $I$ is the stabilizer of a complete lattice chain, and an Iwahori subalgebra $\mathfrak{I}$ is the Lie algebra of $I$.

For more details on lattice chains and the corresponding parahoric subgroups and subalgebras, see for example [14, 2].

There are natural filtrations on $P$ (resp. $\mathfrak{P}$ ) by congruence subgroups (resp. ideals). For $r \in \mathbb{Z}$, define the $\mathfrak{P}$-module $\mathfrak{P}^{r}$ to consist of $X \in \mathfrak{P}$ such that $X L^{i} \subset$ $L^{i+r}$ for all $i$; it is an ideal of $\mathfrak{P}$ for $r \geq 0$ and a fractional ideal otherwise. The congruence subgroup $P^{r} \subset P$ is then defined by $P^{0}=P$ and $P^{r}=\mathrm{I}_{n}+\mathfrak{P}^{r}$ for $r>0$. Note that these ideals are multiplicative, in the sense that $\mathfrak{P}^{r} \mathfrak{P}^{s}=\mathfrak{P}^{r+s}$. If we fix a form $\nu \in \Omega_{F / k}^{1}$ of order -1 , the pairing

$$
\langle X, Y\rangle_{\nu}=\operatorname{Res} \operatorname{Tr}(X Y \nu)
$$

identifies $\mathfrak{P}^{-r}$ with $\left(\mathfrak{P}^{r+1}\right)^{\perp}$ and $\left(\mathfrak{P}^{r+1} / \mathfrak{P}^{s+1}\right)^{\vee}$ with $\mathfrak{P}^{-s} / \mathfrak{P}^{-r}$. There are similar formulas for $\nu$ of arbitrary order, for example, $\left(\mathfrak{P}^{r+1}\right)^{\perp} \cong \mathfrak{P}^{-r+(1+o r d(\nu)) e}$. Throughout this section, we will assume for simplicity that $\operatorname{ord}(\nu)=-1$, but all definitions and results can be stated for other $\nu$.

Definition 2.2. Let $\hat{V}$ be an $F$ vector space, and let $(P, r, \beta)$ be a triple consisting of

- $P \subset \mathrm{GL}(\hat{V})$ a uniform parahoric subgroup;

- $r \in \mathbb{Z}_{\geq 0}$, with $\operatorname{gcd}(r, e)=1$;

- $\beta \in\left(\mathfrak{P}^{r} / \mathfrak{P}^{r+1}\right)^{\vee}$.

After fixing $\nu$ as above, we may identify $\beta$ with a coset $\beta_{\nu}+\mathfrak{P}^{-r+1} \subset \mathfrak{P}^{-r} / \mathfrak{P}^{-r+1}$. Thus, we may choose a representative $\beta_{\nu} \in \mathfrak{P}^{-r}$ for $\beta$. We say that $(P, r, \beta)$ is a uniform stratum if $\beta_{\nu}+\mathfrak{P}^{-r+1}$ contains no nilpotent elements.

In this paper, we are interested in strata that satisfy a 'graded' version of regular semisimplicity. Fix a totally ramified field extension $E / F$ of degree $e$, and let $\mathfrak{o}_{E}$ be the corresponding integral domain. Let

$$
T \cong\left(E^{\times}\right)^{n / e} \subset \mathrm{GL}(\hat{V})
$$

be a maximal torus and let $\mathfrak{t} \cong E^{n / e} \subset \mathfrak{g l}(\hat{V})$ be the corresponding Cartan subalgebra. We denote the identity elements of the Wedderburn components of $\mathfrak{t}$ by $\chi_{j}$, and we write $T(\mathfrak{o})($ resp. $\mathfrak{t}(\mathfrak{o}))$ for $\left(\mathfrak{o}_{E}^{\times}\right)^{n / e} \subset T\left(\right.$ resp. $\left.\mathfrak{o}_{E}^{n / e} \subset \mathfrak{t}\right)$. Moreover, write $\mathfrak{t}^{\mathfrak{b}}$ for the $k$-span of the $\chi_{j}$ and $T^{\mathrm{b}}$ for $\left(\mathfrak{t}^{\mathrm{b}}\right)^{\times}$.

Now, suppose that $(P, r, \beta)$ is a uniform stratum. There is a map $\partial_{\beta, s}: \mathfrak{P}^{s} / \mathfrak{P}^{s+1} \rightarrow$ $\mathfrak{P}^{s-r} / \mathfrak{P}^{s-r+1}$ given by $\partial_{\beta, s}\left(X+\mathfrak{P}^{s}\right)=\operatorname{ad}(X)\left(\beta_{\nu}\right)+\mathfrak{P}^{s-r+1}$.

Definition 2.3. We say that $(P, r, \beta)$ is a regular stratum centralized by $T$ if it satisfies the following conditions:

(1) $T(\mathfrak{o}) \subset P$;

(2) $\operatorname{ker}\left(\partial_{\beta, s}\right)=\mathfrak{t} \cap \mathfrak{P}^{s}+\mathfrak{P}^{s+1}$ for all $s$; 
(3) $y_{\beta}=z^{r} \beta_{\nu}^{e}+\mathfrak{P}^{1}$ is a semi-simple element of the algebra $\mathfrak{P} / \mathfrak{P}^{1}$;

(4) when $r=0$ (and hence, $e_{P}=1$ ), the eigenvalues of $y_{\beta}$ are distinct modulo $\mathbb{Z}$.

In fact, by [2, Theorem 3.6], $\mathcal{L}$ induces a complete lattice chain on each $\chi_{j}(\hat{V})$ (with period $e$ ). Write $I_{j}$ for the corresponding Iwahori subgroup. It follows from 2. Lemma 2.4] that it is the unique lattice chain $\mathcal{L}_{j}$ with $\mathfrak{o}_{E}^{\times} \subset I_{j}$. Moreover, if we fix a uniformizer $\varpi_{E}$ for $\mathfrak{o}_{E}$, then $\varpi_{E} \mathfrak{I}_{j}=\mathfrak{I}_{j}^{1}$. By [3] Proposition 1.18], we deduce that the matrix $\varpi_{T}=\left(\varpi_{E}, \ldots, \varpi_{E}\right) \in \mathfrak{t}$ satisfies the property $\varpi_{T} \mathfrak{P}=\mathfrak{P}_{T}=\mathfrak{P}^{1}$.

Recall the following proposition:

Proposition 2.4 ([2, Proposition 2.10]). Let $(P, r, \beta)$ be a regular stratum centralized by $T$, and let $\beta_{\nu} \in \mathfrak{P}^{-r}$ be a representative for $\beta$. There is a morphism of $\mathfrak{t}$-modules $\pi_{\mathfrak{t}}: \mathfrak{g l}(\hat{V}) \rightarrow \mathfrak{t}$ satisfying the following properties:

(1) $\pi_{\mathfrak{t}}$ restricts to the identity on $\mathfrak{t}$;

(2) $\pi_{\mathfrak{t}}\left(\mathfrak{P}^{\ell}\right) \subset \mathfrak{P}^{\ell}$;

(3) the kernel of the induced map

$$
\bar{\pi}_{\mathfrak{t}}:\left(\mathfrak{t}+\mathfrak{P}^{\ell-r}\right) / \mathfrak{P}^{\ell-r+1} \rightarrow \mathfrak{t} /\left(\mathfrak{t} \cap \mathfrak{P}^{\ell-r+1}\right)
$$

is given by the image of $\operatorname{ad}\left(\mathfrak{P}^{\ell}\right)\left(\beta_{\nu}\right)$ modulo $\mathfrak{P}^{\ell-r+1}$;

(4) if $y \in \mathfrak{t}$ and $X \in \mathfrak{g l}(\hat{V})$, then $\langle y, X\rangle_{\nu}=\left\langle y, \pi_{\mathfrak{t}}(X)\right\rangle_{\nu}$;

(5) let $\bar{\pi}_{\mathfrak{t}, \ell}: \mathfrak{P}^{\ell} / \mathfrak{P}^{\ell+1} \rightarrow \mathfrak{t} / \mathfrak{t} \cap \mathfrak{P}^{\ell+1}$ be the induced map, and set $W_{\ell}=\operatorname{ker}\left(\bar{\pi}_{\mathfrak{t}, \ell}\right)$. Then, the induced map $\operatorname{ad}\left(\beta_{\nu}\right): W_{\ell} \rightarrow W_{\ell-r}$ is an isomorphism.

A connection on $\hat{V}$ is a $k$-derivation $\nabla: \hat{V} \rightarrow \hat{V} \otimes \Omega_{F / k}^{1}$. If $\tau$ is a $k$-derivation on $F$, we write $\nabla_{\tau}$ for the composition of $\nabla$ with the inner derivation associated to $\tau: \nabla_{\tau}(v)=i_{\tau}(\nabla(v))$. In particular, let $\tau_{\nu}$ be the derivation with the property that $i_{\tau_{\nu}}(\nu)=1$.

Definition 2.5. Let $(P, r, \beta)$ be a uniform stratum. When $r \geq 1$, we say that $(\hat{V}, \nabla)$ contains $(P, r, \beta)$ if $\nabla_{\tau_{\nu}}\left(L^{i}\right) \subset L^{i-r}$ and $\left(\nabla_{\tau_{\nu}}-\beta_{\nu}\right)\left(L^{i}\right) \subset L^{i-r+1}$ for all $i$. When $r=0$, and thus $e=1$, we say that $(\hat{V}, \nabla)$ contains $(P, 0, \beta)$ if $\left(\nabla_{\tau_{\nu}}-\beta_{\nu}\right)\left(L^{i}\right) \subset L^{i+1}$ for some lattice $L^{i}$.

Given a trivialization $\phi: \hat{V} \stackrel{\sim}{\rightarrow} F^{n}$, we write $[\nabla]_{\phi}$ for the matrix of $\nabla$ with respect to the standard basis of $F^{n}$. By the Leibniz rule, $\nabla=d_{z}+[\nabla]_{\phi}$ where $d_{z}$ is the usual exterior $k$-differential on $F$. The group $\mathrm{GL}_{n}(F)$ acts transitively on the space of trivializations for $F$, and

$$
[\nabla]_{g \phi}=g \cdot[\nabla]_{\phi}:=\operatorname{Ad}(g)[\nabla]_{\phi}-\left(d_{z} g\right) g^{-1} .
$$

If we have fixed a base trivialization, we will shorten $[\nabla]_{g \phi}$ to $[\nabla]_{g}$ and $[\nabla]_{\phi}$ to $[\nabla]$. In general, the left action $g \cdot$ on $\mathfrak{g l}_{n}(F) \otimes \Omega_{F / k}^{1}$ is called a gauge transformation, and we say that two matrices $X, Y \in \mathfrak{g l}_{n}(F) \otimes \Omega_{F / k}^{1}$ are gauge equivalent if there exists $g \in \mathrm{GL}_{n}(F)$ such that $g \cdot X=Y$. Thus, if $\nabla$ and $\nabla^{\prime}$ are connections on $\hat{V}$, and $[\nabla]_{\phi}$ is gauge equivalent to $\left[\nabla^{\prime}\right]_{\phi}$, then $(\hat{V}, \nabla)$ and $\left(\hat{V}, \nabla^{\prime}\right)$ are isomorphic.

Now, suppose that $(P, r, \beta)$ is a regular stratum in $\mathrm{GL}_{n}(F)$ centralized by a torus $T$. We denote the pullback of $P$ and $\beta$ to $\operatorname{GL}(\hat{V})$ by $P^{\phi}$ and $\beta^{\phi}$, respectively.

Theorem 2.6. [2, Theorem 4.13] If $(\hat{V}, \nabla)$ contains the stratum $\left(P^{\phi}, r, \beta^{\phi}\right)$, then there exists $p \in P^{1}$ and a regular element $A_{\nu} \in \mathfrak{t} \cap \mathfrak{P}^{-r}$ such that $p \cdot[\nabla]_{\phi}=A_{\nu} \nu$. Furthermore, the orbit of $A_{\nu} \nu$ under $P^{1}$-gauge transformations contains $\left(A_{\nu}+\mathfrak{P}^{1}\right) \nu$. 
Remark 2.7. By [2, Lemma 4.4], the map $\mathfrak{P}^{-r} \rightarrow \mathfrak{P}^{-r} / \mathfrak{P}^{1}$ intertwines the gauge and adjoint actions of $P$. This implies that the functional induced on $\mathfrak{P}^{r} / \mathfrak{P}^{r+1}$ by $A_{\nu}$ coincides with $\beta$.

We now define the "formal type" of a connection.

Definition 2.8. Let $A \in \mathfrak{P}^{\vee} \backslash\{0\}$, and suppose that $\mathfrak{P}^{r+1}$ is the smallest congruence ideal contained in $A^{\perp}$. Let $\beta$ be the restriction of $A$ to $\mathfrak{P}^{r} / \mathfrak{P}^{r+1}$. We say that $A$ is a formal type if it satisfies the following conditions:

(1) the stratum $(P, r, \beta)$ is regular and centralized by $T$, and

(2) any representative $A_{\nu} \in \mathfrak{P}^{-r}$ for $A$ lies in $\mathfrak{t}+\mathfrak{P}^{1}$.

The connection $(\hat{V}, \nabla)$ has formal type $A$ if there is a trivialization $\phi: \hat{V} \rightarrow$ $F^{n}$ such that $(\hat{V}, \nabla)$ contains the stratum $\left(P^{\phi}, r, \beta^{\phi}\right)$, and $[\nabla]_{\phi}$ is formally gauge equivalent to an element of $\left(A_{\nu}+\mathfrak{P}^{1}\right) \nu$ by an element of $P^{1}$. Finally, we say that two connections that contain regular strata are combinatorially equivalent if the strata have the same parahoric subgroup and slope.

By [2, Corollary 4.16], any two connections with formal type $A$ are formally isomorphic, and the formal type is independent of $\nu$. The converse is false, since any conjugate of $A$ by the relative Weyl group of $T$ is also a formal type for $(\hat{V}, \nabla)$. However, fixing a stratum uniquely determines the formal type. For the rest of the section, we assume (without loss of generality) that $P \subset \mathrm{GL}_{n}(\mathfrak{o})$.

Proposition 2.9. If $\nabla$ contains a regular stratum $\left(P^{\phi}, r, \beta^{\phi}\right)$, there exists a unique formal type $A$ such that $\left.A\right|_{\mathfrak{P}^{r}}=\beta$ and $[\nabla]_{\phi}$ is gauge equivalent to an element of $\left(A_{\nu}+\mathfrak{P}^{1}\right) \nu$ by an element of $\mathrm{GL}_{n}(\mathfrak{o})$.

Proof. By Theorem 2.6 and Remark 2.7 $(\hat{V}, \nabla)$ has a formal type $A$ such that $\left.A\right|_{\mathfrak{P}^{r}}=\beta$, and there exists $p \in P^{1}$ such that $p \cdot[\nabla]_{\phi}=A_{\nu} \nu$, where $A_{\nu} \in \mathfrak{t} \cap \mathfrak{P}^{-r}$. This formal type is unique, since [2, Lemma 3.16] implies that if $[\nabla]_{\phi}$ is gauge equivalent to $A_{\nu}^{\prime} \in \mathfrak{t} \cap \mathfrak{P}^{-r}$ and $\left.A_{\nu}^{\prime}\right|_{\mathfrak{P}^{r}}=\beta$, then $A_{\nu}^{\prime}+\mathfrak{P}^{1}=A_{\nu}+\mathfrak{P}^{1}$.

Finally, throughout the paper we will need to consider a slight variation on the formal type. Choose a uniformizer $\varpi_{E}$ for $E$ such that $\varpi_{E}^{e}=z$; under a suitable embedding $E \hookrightarrow \mathfrak{g l}_{n / e}(\mathbb{C})$, such a way that

$$
\varpi_{E}=\left(\begin{array}{cccc}
0 & 1 & \cdots & 0 \\
\vdots & \ddots & \ddots & \vdots \\
0 & \ddots & 0 & 1 \\
z & 0 & \cdots & 0
\end{array}\right) .
$$

We choose a basis for each $\chi_{j} \hat{V}$ (and hence for $V$ ) such that $\varpi_{T}$ is block diagonal with these blocks. Using this basis, we define $H_{T}=\left(H_{E}, \ldots, H_{E}\right) \in \mathfrak{P}$ as the block diagonal matrix with blocks given by the diagonal matrix $H_{E}=$ $\operatorname{diag}\left(\frac{e-1}{2 e}, \frac{e-3}{2 e}, \ldots, \frac{1-e}{2 e}\right)$. Let $H_{T}^{\prime} \in \mathfrak{P}^{\vee}$ to be the corresponding functional $H_{T}^{\prime}(X)=$ $\left.\operatorname{Tr}\left(H_{T} X\right)\right|_{z=0}$.

Definition 2.10. Let $(\hat{V}, \nabla)$ have formal type $A$. We define the normalized formal type of $(\hat{V}, \nabla)$ to be $\tilde{A}=A+H_{T}^{\prime}$.

Note that if $e=1$, then $\tilde{A}=A$. 
Proposition 2.11. Suppose that $(\hat{V}, \nabla)$ contains a regular stratum and has normalized formal type $\tilde{A}$. If $\tilde{A}_{\nu} \in \mathfrak{P}^{-r}$ is a representative for $\tilde{A}$, then there exists $\hat{p} \in P^{1}$ such that $\hat{p} \cdot[\nabla]_{\phi}=\tilde{A}_{\nu} \nu$.

Proof. If $r=0$ (so $e=1$ ), this is just Theorem [2.6. so assume that $r \geq 1$. Note that $H_{T}^{\prime} \in\left(\mathfrak{t}^{\mathfrak{b}}\right)^{\perp}$. Therefore, part 4 of Proposition 2.4 imply that $\pi_{\mathfrak{t}}\left(H_{T}\right) \in$ $\mathfrak{P}^{1}$. Part 3 of the same proposition shows that there exists $X \in \mathfrak{P}^{r}$ such that $\operatorname{ad}(X) \tilde{A}_{\nu} \in H_{T} \frac{1}{\operatorname{Res}(\nu)}+\mathfrak{P}^{1}$, so there exists $p \in P^{1}$ such that $p \cdot[\nabla]_{\phi}=A_{\nu} \nu$ by Theorem 2.6. Moreover, $\exp (-X) \cdot \tilde{A}_{\nu} \nu \in\left(A_{\nu}+\mathfrak{P}^{1}\right) \nu$, so there exists $p^{\prime} \in P^{1}$ such that $p^{\prime} \exp (-X) \cdot \tilde{A}_{\nu} \nu=A_{\nu} \nu$. It follows that $\left(\exp (X)\left(p^{\prime}\right)^{-1} p\right) \cdot[\nabla]_{\phi}=\tilde{A}_{\nu} \nu$.

Proposition 2.12. Any normalized formal type $\tilde{A}$ is stabilized by $T \cap P^{1}$.

Proof. Since ad $\left(\mathfrak{P}^{1}\right) H_{T} \in \mathfrak{P}^{1}, P^{1}$ stabilizes $H_{T}+\mathfrak{P}^{1}$. Moreover, the corresponding formal type $A$ is stabilized by $T$.

\section{Moduli SPACes of CONNECTiOns}

In this section, we will describe the moduli space of connections on $\mathbb{P}^{1}(\mathbb{C})$ with compatible framings and fixed combinatorics at each singular point.

First, we recall the construction of the moduli space of framed connections on $\mathbb{P}^{1}(\mathbb{C})$ with fixed formal type at each singular point 2 . Throughout, $I$ will be a finite indexing set and $\mathbf{x}=\left\{x_{i}\right\}_{i \in I}$ a collection of distinct points in $\mathbb{P}^{1}$. We denote the completion of the function field of $\mathbb{P}^{1}$ at $x_{i}$ by $F_{i}$ and the corresponding power series ring by $\mathfrak{o}_{i}$.

Let $V$ be a trivial rank $n$ vector bundle on $\mathbb{P}^{1}$, i.e., we have fixed a trivialization $V \cong \mathcal{O}_{\mathbb{P}^{1}}^{n}$. Accordingly, we may identify the space of all global trivializations of $V$ with $\mathrm{GL}_{n}(\mathbb{C})$. Note that a global trivialization determines a local trivialization of $V_{i}:=V \otimes_{\mathcal{O}_{\mathbb{P} 1}} F_{i}$, so there is a natural inclusion $\mathrm{GL}_{n}(\mathbb{C}) \hookrightarrow \mathrm{GL}_{n}\left(F_{i}\right)$; moreover, the global sections of $V$ generate a distinguished lattice $L_{i} \subset V_{i}$. Suppose that $\nabla$ is a connection on $V$ with the property that the induced connection on $V_{i}$ has formal type $A_{i}$. We will assume without loss of generality that the associated torus $T_{i}$ is contained in $\mathrm{GL}_{n}\left(\mathfrak{o}_{i}\right)$. Therefore, by [2, Proposition 4.14], $A_{i}$ determines a unique stratum $\left(P_{i}, r, \beta_{i}\right)$ in $\mathrm{GL}_{n}\left(F_{i}\right)$. We also set $\varpi_{i}=\varpi_{T_{i}}$.

Throughout this section, $U_{i}$ will denote the unipotent subgroup $P_{i}^{1} \cap \mathrm{GL}_{n}(\mathbb{C})$ with Lie algebra $\mathfrak{u}_{i}=\mathfrak{P}_{i}^{1} \cap \mathfrak{g l} \mathfrak{l}_{n}(\mathbb{C})$. For simplicity, we write $\mathfrak{g}_{i}$ for the parahoric subalgebra $\mathfrak{g l}_{n}\left(\mathfrak{o}_{i}\right) ;$ its radical is $\mathfrak{g}_{i}^{1}=t \mathfrak{g}_{i}$. Note that $U_{i} \cong P_{i}^{1} /\left(1+\mathfrak{g}_{i}^{1}\right)$ and $\mathfrak{u}_{i} \cong \mathfrak{P}_{i}^{1} / \mathfrak{g}^{1}$.

Definition 3.1. A compatible framing for $\nabla$ at $x_{i}$ is a global trivialization $g \in$ $\mathrm{GL}_{n}(\mathbb{C})$ with the property that $\nabla$ contains the $\operatorname{GL}\left(V_{i}\right)$-stratum $\left(P_{i}^{g}, r, \beta_{i}^{g}\right)$ defined above. We say that $\nabla$ is framed at $x_{i}$ if there exists such a $g$.

We now define the moduli space of connections with fixed formal type and a specified framing at each singular point. Set $\bar{A}_{i}=\left.A_{i}\right|_{\mathfrak{P}^{1}}$.

Definition 3.2. Let $\widetilde{\mathcal{M}}(\mathbf{A})$ be the the moduli space of isomorphism classes of triples $(V, \nabla, \mathbf{g})$, where

- $\nabla$ is a meromorphic connection on the trivial bundle $V$ with singularities at $\left\{x_{i}\right\}_{i \in I}$;

- $\mathbf{g}=\left\{U_{i} g_{i}\right\}_{i \in I}$, with $g_{i}$ a compatible framing for $\nabla$ at $x_{i}$;

- the formal type $A_{i}^{\prime}$ of $\nabla$ at $x_{i}$ satisfies $\bar{A}_{i}^{\prime}=\bar{A}_{i}$. 
The moduli space $\widetilde{\mathcal{M}}(\mathbf{A})$ is built out of "extended" coadjoint orbits $\widetilde{\mathcal{M}}\left(A_{i}\right)$ determined locally by each formal type $A_{i}$. In the following, let $A$ be a formal type. We define $\pi_{\mathfrak{P}}: \mathfrak{g}^{\vee} \rightarrow \mathfrak{P}^{\vee}$ and $\pi_{\mathfrak{P}^{1}}: \mathfrak{g}^{\vee} \rightarrow\left(\mathfrak{P}^{1}\right)^{\vee}$ to be the restriction maps and $\mathcal{O}^{1}$ to be the orbit of $\bar{A}$ under the coadjoint action of $P^{1}$. Also, when $r=0$ (so $\left.e=1\right)$, we take $\left(\mathfrak{t}^{b}\right)^{\prime} \subset \mathfrak{g l}_{n}(\mathbb{C})^{\vee}$ to be the set of functionals of the form $\phi(X)=\operatorname{Tr}(D X)$, where $D \in \mathfrak{t}^{b}$ is a diagonal matrix with distinct eigenvalues modulo $\mathbb{Z}$.

Definition 3.3. When $r>0$, define the extended orbit $\widetilde{\mathcal{M}}(A) \subset\left(U \backslash \mathrm{GL}_{n}(\mathbb{C})\right) \times \mathfrak{g}^{\vee}$ to be

$$
\left.\widetilde{\mathcal{M}}(A)=\left\{(U g, \alpha) \mid \pi_{\mathfrak{P}^{1}}\left(\operatorname{Ad}^{*}(g)(\alpha)\right) \in \mathcal{O}^{1}\right)\right\} .
$$

When $r=0$, we define $\widetilde{\mathcal{M}}(A)$ by

$$
\widetilde{\mathcal{M}}(A)=\left\{(g, \alpha) \in \mathrm{GL}_{n}(\mathbb{C}) \times \mathfrak{g l}_{n}(\mathbb{C})^{\vee} \mid \operatorname{Ad}^{*}(g) \alpha \in\left(\mathfrak{t}^{\mathfrak{b}}\right)^{\prime}\right\} .
$$

There is a natural action $\rho$ of $\mathrm{GL}_{n}(\mathbb{C})$ on $\widetilde{\mathcal{M}}(A)$ given by $\rho(h)(U g, \alpha)=\left(U g h^{-1}, \operatorname{Ad}^{*}(h) \alpha\right)$.

Note that when $r=0, \widetilde{\mathcal{M}}(A)$ is independent of $A$.

Proposition 3.4. [2, Proposition 5.10] The space $\widetilde{\mathcal{M}}(A)$ is a symplectic manifold, and $\rho$ is a Hamiltonian action. The moment map for $\rho$ is given by $\mu_{\rho}(U g, \alpha)=$ $\operatorname{res}(\alpha)$, where $\operatorname{res}(\alpha)=\left.\alpha\right|_{\mathfrak{g l}_{n}(\mathbb{C})}$.

Remark 3.5. If $\alpha_{\nu}$ is any representative of $\alpha$, then $\operatorname{res}(\alpha)=\operatorname{Res}\left(\alpha_{\nu} \nu\right)$.

Theorem 3.6. [2, Theorem 5.4] The moduli space $\widetilde{\mathcal{M}}(\mathbf{A})$ is the symplectic reduction of $\prod_{i \in I} \widetilde{\mathcal{M}}\left(A_{i}\right)$ by the diagonal action of $\mathrm{GL}_{n}(\mathbb{C})$ :

$$
\widetilde{\mathcal{M}}(\mathbf{A}) \cong\left(\prod_{i \in I} \widetilde{\mathcal{M}}\left(A_{i}\right)\right) / / 0 \mathrm{GL}_{n}(\mathbb{C}) .
$$

Moreover, $\widetilde{\mathcal{M}}(\mathbf{A})$ is a symplectic manifold.

Specifically, $\prod_{i \in I} \widetilde{\mathcal{M}}\left(A_{i}\right)$ is a symplectic manifold, and the diagonal action of $\mathrm{GL}_{n}(\mathbb{C})$ has moment map $\mu_{\mathrm{GL}_{n}}=\sum_{i \in I}$ res $_{i}$. Thus,

$$
\left(\prod_{i \in I} \widetilde{\mathcal{M}}\left(A_{i}\right)\right) / / 0 \mathrm{GL}_{n}(\mathbb{C})=\mu_{\mathrm{GL}_{n}}^{-1}(0) / \mathrm{GL}_{n}(\mathbb{C})
$$

In the remainder of this section, we describe a larger moduli space in which we fix only the combinatorics of $\left(V_{i}, \nabla_{i}\right)$ at $x_{i}$, and not the formal type. Again, it will be constructed as a reduction of the product of local pieces.

First, we discuss these local pieces. If $P$ is a uniform parahoric subgroup $P$ with period $e, r \geq 0$ is an integer such that $(r, e)=1$, and $T$ is a maximal torus such that $T(\mathfrak{o}) \subset P$, we let $\left(\mathfrak{P} / \mathfrak{P}^{r+1}\right)_{\text {reg }}^{\vee}$ be the set of $\gamma \in\left(\mathfrak{P} / \mathfrak{P}^{r+1}\right)^{\vee}$ such that $\left(P, r,\left.\gamma\right|_{\mathfrak{P}^{r} / \mathfrak{P}^{r+1}}\right)$ is a regular stratum centralized by $T$. We also let $\mathcal{A}(P, r)$ be the subset of $\left(\mathfrak{P} / \mathfrak{P}^{r+1}\right)_{\text {reg }}^{\vee}$ consisting of normalized formal types. Note that $\mathcal{A}(P, r)$ is the subset of $\left(\mathfrak{P} / \mathfrak{P}^{r+1}\right)_{\text {reg }}^{\vee}$ consisting of $X+H_{T}$, where $X$ is stabilized by the coadjoint action of $T(\mathfrak{o})$. We also define $\left(\mathfrak{P}^{1} / \mathfrak{P}^{r+1}\right)_{\text {reg }}^{\vee}$ to be the projection of $\left(\mathfrak{P} / \mathfrak{P}^{r+1}\right)_{\text {reg }}^{\vee}$ onto $\left(\mathfrak{P}^{1} / \mathfrak{P}^{r+1}\right)^{\vee}$.

Definition 3.7. If $r=0$, define $\widetilde{\mathcal{M}}(P, r)=\widetilde{\mathcal{M}}(A)$ for any $A$. If $r>0$, define $\widetilde{\mathcal{M}}(P, r) \subset\left(U \backslash \mathrm{GL}_{n}(\mathbb{C})\right) \times \mathfrak{g l}_{n}(\mathfrak{o})^{\vee}$ by

$$
\widetilde{\mathcal{M}}(P, r)=\left\{(U g, \alpha) \mid \pi_{\mathfrak{P}}\left(\operatorname{Ad}^{*}(g) \alpha\right) \in\left(\mathfrak{P} / \mathfrak{P}^{r+1}\right)_{\text {reg }}^{\vee}\right\} .
$$


The space $\widetilde{\mathcal{M}}(P, r)$ is a manifold; the argument is similar to the proof that $\widetilde{\mathcal{M}}(A)$ is smooth given in [2].

Set $\mathfrak{V}=\pi_{\mathfrak{P}}^{-1}\left(\mathfrak{P} / \mathfrak{P}^{r+1}\right)_{\text {reg }}^{\vee} \subset\left(\mathfrak{g} / \mathfrak{P}^{r+1}\right)^{\vee}$. In the notation of Proposition 2.4 part (5), let $\widetilde{W}_{r} \subset \mathfrak{P}^{r}$ be the subset of elements that map to $W_{r}\left(\bmod \mathfrak{P}^{r+1}\right)$. We define $Z=\left(\mathfrak{g} / \widetilde{W}_{r}\right)^{\vee}$. Note that in the case $r=0, \mathfrak{V}=\left(\mathfrak{g} / \mathfrak{g}^{1}\right)_{\text {reg }}^{\vee} \cong\left(\mathfrak{t}^{\mathfrak{b}}\right)^{\prime}=$ $\mathcal{A}\left(\mathrm{GL}_{n}(\mathfrak{o}), 0\right)$ and $Z \cong \mathfrak{t}^{\mathfrak{b}}$.

Lemma 3.8. There is an isomorphism $\mathfrak{V} \times_{U} \mathrm{GL}_{n}(\mathbb{C}) \cong \widetilde{\mathcal{M}}(P, r)$. Furthermore, there are open dense inclusions $\left(\mathfrak{P} / \mathfrak{P}^{r+1}\right)_{\mathrm{reg}}^{\vee} \hookrightarrow\left(\mathfrak{P} / \widetilde{W}_{r}\right)^{\vee}, \mathfrak{V} \hookrightarrow Z$, and $\left(\mathfrak{P}^{1} / \mathfrak{P}^{r+1}\right)_{\mathrm{reg}}^{\vee} \hookrightarrow$ $\left(\mathfrak{P}^{1} / \widetilde{W}_{r}\right)^{\vee}$ in the case $r \geq 1$.

Proof. The first isomorphism is given by the map $(v, g) \mapsto\left(U g, \operatorname{Ad}^{*}\left(g^{-1}\right) v\right)$. Next, we observe that $\left(\mathfrak{P} / \mathfrak{P}^{r+1}\right)_{\text {reg }}^{\vee}$ may be identified with an open subset of $\left(\mathfrak{t} \cap \mathfrak{P}^{-r}+\right.$ $\left.\mathfrak{P}^{-r+1}\right) / \mathfrak{P}^{1}$. By part (4) of Proposition 2.4, $\widetilde{W}_{r}=\left(\mathfrak{t} \cap \mathfrak{P}^{-r}+\mathfrak{P}^{-r+1}\right)^{\perp}$. It follows that $\left(\mathfrak{t} \cap \mathfrak{P}^{-r}+\mathfrak{P}^{-r+1}\right) / \mathfrak{P}^{1} \cong\left(\mathfrak{P} / \widetilde{W}_{r}\right)^{\vee}$. The other inclusions are proved similarly.

Proposition 3.9. There is a smooth map $\Gamma: \widetilde{\mathcal{M}}(P, r) \rightarrow \mathcal{A}(P, r)$ which assigns to $(U g, \alpha)$ the unique normalized formal type in the $P^{1}$-orbit of $\pi_{\mathfrak{P}}(\operatorname{Ad}(g)(\alpha))$. The fiber $\Gamma^{-1}(\tilde{A})$ is isomorphic to $\widetilde{\mathcal{M}}(A)$.

Proof. Using the description of $\widetilde{\mathcal{M}}(P, r)$ in Lemma 3.8 , we will construct a smooth map $\zeta: \mathfrak{V} \times \mathrm{GL}_{n}(\mathbb{C}) \rightarrow \mathcal{A}(P, r)$ and then show that it $U$-equivariant.

First, we show that

$$
\left(\mathfrak{P} / \mathfrak{P}^{r+1}\right)_{\mathrm{reg}}^{\vee} \cong \mathcal{A}(P, r) \times_{T \cap P^{1}} P^{1} / P^{r+1},
$$

where $T \cap P^{1}$ acts on the right factor by left translation, and on the left factor by the coadjoint action. There is a natural map $\mathcal{A}(P, r) \times_{T \cap P^{1}} P^{1} / P^{r+1} \rightarrow\left(\mathfrak{P} / \mathfrak{P}^{r+1}\right)_{\text {reg }}^{\vee}$ given by $(Y, \bar{p}) \mapsto \operatorname{Ad}^{*}\left(p^{-1}\right) Y$. The inverse map takes a regular functional $\gamma$ to the class of $(A, \bar{p})$, where $\tilde{A}$ is the unique normalized formal type in the $P^{1}$-orbit of $\tilde{A}$ and $\tilde{A}=\operatorname{Ad}^{*}(p) \gamma$.

We now define $\zeta^{\prime}:\left(\mathfrak{P} / \mathfrak{P}^{r+1}\right)_{\text {reg }}^{\vee} \rightarrow \mathcal{A}(P, r)$ as the projection onto the left factor of $\mathcal{A}(P, r) \times_{T \cap P^{1}} P^{1} / P^{r+1}$; this makes sense since the coadjoint action of $T \cap P^{1}$ on $\mathcal{A}(P, r)$ is trivial by Proposition 2.12. In particular, it is clear that $\zeta^{\prime}\left(\operatorname{Ad}^{*}(u) X\right)=$ $X$ for any $u \in U$. It follows that the map $\zeta(v, g)=\zeta^{\prime}\left(\pi_{\mathfrak{P}}(v)\right)$ is $U$-equivariant, where $U$ acts trivially on $\mathcal{A}(P, r)$. We define $\Gamma$ to be the map induced by $\zeta$ on $\mathfrak{V} \times_{U} \mathrm{GL}_{n}(\mathbb{C})$.

Finally, we see that $\widetilde{\mathcal{M}}(A)$ embeds into $\Gamma^{-1}(\tilde{A})$ by comparing Definitions 3.3 and 3.7. Moreover, if $(U g, \alpha) \in \Gamma^{-1}(\tilde{A})$, then $\pi_{\mathfrak{P}^{1}}\left(\operatorname{Ad}^{*}(g)(\alpha)\right) \in \mathcal{O}^{1}$. Therefore, $\widetilde{\mathcal{M}}(A) \cong \Gamma^{-1}(\tilde{A})$.

Before proceeding, we need to recall some facts about Poisson reduction. Recall that if a Lie group $G$ acts on a Poisson manifold $M$ via a canonical Poisson action, then there is a corresponding moment map $\mu_{M}: M \rightarrow \mathfrak{g}^{\vee}$. The following result appears in [11.

Proposition 3.10 ([11, Examples 3.B, 3.F]). Let $M$ be a Poisson manifold, and suppose that $G$ is a linear algebraic group with a free canonical Poisson action on 
$M$. If 0 is a regular value for $\mu_{M}$, then the Poisson structure on $M$ induces a Poisson structure on $M / /{ }_{0} G \stackrel{\text { def }}{=} \mu^{-1}(0) / G$ called the Poisson reduction of $M$. If the symplectic leaves of $M$ intersect the $G$-orbits cleanly (in the terminology of 6 , II.25, p. 180]), then the symplectic leaves of $M / /{ }_{0} G$ are the connected components of the symplectic reductions of those symplectic leaves of $M$ that intersect $\mu^{-1}(0)$.

Lemma 3.11. When $r \geq 1$, $\left(\mathfrak{P}^{1} / \mathfrak{P}^{r+1}\right)_{\text {reg }}^{\vee}$ has a natural Poisson structure.

Proof. By Lemma 3.8, $\left(\mathfrak{P}^{1} / \mathfrak{P}^{r+1}\right)_{\text {reg }}^{\vee}$ is naturally isomorphic to an open dense subset of $\left(\mathfrak{P}^{1} / \widetilde{W}_{r}\right)^{\vee}$. It suffices to show that $\left(\mathfrak{P}^{1} / \widetilde{W}_{r}\right)^{\vee}$ is a Poisson manifold. Since $\left[\mathfrak{P}^{1}, \widetilde{W}_{r}\right] \subset \mathfrak{P}^{r+1}$, and the Poisson bracket restricted to linear functions on $\left(\mathfrak{P}^{1} / \mathfrak{P}^{r+1}\right)^{\vee}$ is just the usual Lie bracket on $\mathfrak{P}^{1} / \mathfrak{P}^{r+1}$, this implies that the ideal generated by $W_{r} \subset \mathfrak{P}^{1} / \mathfrak{P}^{r+1}$ is a Poisson ideal. It follows that $\left(\mathfrak{P}^{1} / \widetilde{W}_{r}\right)^{\vee} \cong W_{r}^{\perp} \subset$ $\left(\mathfrak{P}^{1} / \mathfrak{P}^{r+1}\right)^{\vee}$ is a Poisson space. Therefore $\left(\mathfrak{P}^{1} / \mathfrak{P}^{r+1}\right)_{\text {reg }}^{\vee}$ is Poisson.

Proposition 3.12. The manifold $\widetilde{\mathcal{M}}(P, r)$ has a Poisson structure. When $r \geq 1$, the manifold $\widetilde{\mathcal{M}}(P, r)$ is isomorphic to a Poisson reduction:

$$
\widetilde{\mathcal{M}}(P, r) \cong\left(\left(\mathfrak{P}^{1} / \mathfrak{P}^{r+1}\right)_{r e g}^{\vee} \times T^{*} \mathrm{GL}_{n}(\mathbb{C})\right) / / 0 U .
$$

The symplectic leaves of $\widetilde{\mathcal{M}}(P, r)$ are the fibers of the map $\Gamma$.

Proof. In the case $r=0, \widetilde{\mathcal{M}}(P, r) \cong \widetilde{\mathcal{M}}(A)$ by the remark following Definition 3.7 Therefore, $\widetilde{\mathcal{M}}(P, r)$ is in fact symplectic.

We now suppose that $r \geq 1$. The space $\left.\left(\mathfrak{P}^{1} / \mathfrak{P}^{r+1}\right)_{\text {reg }}^{\vee} \times T^{*} \mathrm{GL}_{n}(\mathbb{C})\right)$ is a Poisson manifold using the Poisson structure of Lemma 3.11 on the first factor and the usual symplectic structure of a cotangent bundle on the second.

The group $U$ acts on $\left(\mathfrak{P}^{1} / \mathfrak{P}^{r+1}\right)_{\text {reg }}^{\vee}$ and $T^{*} \mathrm{GL}_{n}(\mathbb{C})$ via the coadjoint action and the free action induced by left multiplication on $\mathrm{GL}_{n}(\mathbb{C})$ respectively; these actions are canonical Poisson.

The moment map of the diagonal action is given by

$$
\tilde{\mu}(Y,(g, X))=-\left.\operatorname{Ad}^{*}(g)(X)\right|_{\mathfrak{u}}+\left.Y\right|_{\mathfrak{u}} .
$$

It is clear that $\tilde{\mu}$ is a submersion.

If $\tilde{\mu}(Y,(g, X))=0$, then $\left.\operatorname{Ad}^{*}(g)(X)\right|_{\mathfrak{u}}=\left.Y\right|_{\mathfrak{u}}$. Therefore, we may glue $\operatorname{Ad}^{*}(g)(X)$ and $Y$ together to obtain a functional $\psi_{Y, X} \in\left(\mathfrak{g} / \mathfrak{P}^{r+1}\right)^{\vee}$; note that $\psi_{Y, X} \in \mathfrak{V}$ if and only if $Y \in\left(\mathfrak{P}^{1} / \mathfrak{P}^{r+1}\right)_{\text {reg }}^{\vee}$. Using the description of $\widetilde{\mathcal{M}}(P, r)$ in Lemma 3.8, we define a map $p: \tilde{\mu}^{-1}(0) \rightarrow \widetilde{\mathcal{M}}(P, r)$ by

$$
(Y,(g, X)) \mapsto\left(\psi_{Y, X}, g\right) \in\left(\mathfrak{g} / \mathfrak{P}^{r+1}\right)^{\vee} \times_{U} \mathrm{GL}_{n}(\mathbb{C}) .
$$

The map is surjective, and the fibers of $p$ are $U$-orbits.

The symplectic leaves of $\left(\mathfrak{P}^{1} / \mathfrak{P}^{r+1}\right)_{\text {reg }}^{\vee} \times T^{*} \mathrm{GL}_{n}(\mathbb{C})$ are given by $\mathcal{O} \times T^{*} \mathrm{GL}_{n}(\mathbb{C})$, where $\mathcal{O}$ is any coadjoint orbit in $\left(\mathfrak{P}^{1} / \mathfrak{P}^{r+1}\right)_{\text {reg }}^{\vee}$. It is obvious that the $U$-orbits intersect the leaves cleanly. It now follows from Definition 3.3 and the fact that the $\widetilde{\mathcal{M}}(A)$ are connected that the symplectic leaves of $\widetilde{\mathcal{M}}(P, r)$ are given by $\widetilde{\mathcal{M}}(A)$ for $A$ a formal type corresponding to $P$ and $r$.

Lemma 3.13. The $\mathrm{GL}_{n}(\mathbb{C})$-action on $\widetilde{\mathcal{M}}(P, r)$ defined by $h(U g, \alpha)=\left(U g h^{-1}, \mathrm{Ad}^{*}(h)(\alpha)\right)$ is free canonical Poisson with submersive moment map $\mu(U g, \alpha)=\operatorname{res}(\alpha)$. 
Proof. By [2, Lemma 5.12], this action restricts to a free action on each symplectic leaf $\widetilde{\mathcal{M}}(A)$. To see that it is canonical Poisson, first observe that the $\mathrm{GL}_{n}(\mathbb{C})$ action on $\left(\mathfrak{P}^{1} / \mathfrak{P}^{r+1}\right)_{\text {reg }}^{\vee} \times T^{*} \mathrm{GL}_{n}(\mathbb{C})$ given by $h \cdot(Y,(g, X))=\left(Y, g h^{-1}, A d^{*}(h) X\right)$ is canonical Poisson with moment map $(Y,(g, X)) \mapsto X$. Since it commutes with the action of $U$, it induces a canonical Poisson action on the Poisson reduction with moment map given by the same formula. It is easy to check that this action corresponds to the given action on $\widetilde{\mathcal{M}}(P, r)$ under the isomorphism of Proposition 3.12. Since $(Y,(g, X))$ corresponds to $\left(\psi_{Y, X}, g\right)$ and $\operatorname{res}\left(\psi_{Y, X}\right)=X$, we obtain the desired expression for the moment map. Finally, by [2, Lemma 5.11], $\mu$ is even a submersion when restricted to any symplectic leaf.

We are now ready to construct the moduli space of framed connections on $\mathbb{P}^{1}(\mathbb{C})$ with fixed combinatorics. Recall that $\mathbf{x}$ is a finite set of points in $\mathbb{P}^{1}(\mathbb{C})$ indexed by $I$. Let $\mathbf{P}=\left\{P_{i}\right\}_{i \in I}$ be a collection of uniform parahoric subgroups with periods $e_{i}$ such that $P_{i} \in \mathrm{GL}_{n}\left(\mathfrak{o}_{i}\right)$, and let $\mathbf{r}=\left(r_{i}\right)_{i \in I}$ with $r_{i} \geq 0$ and $\operatorname{gcd}\left(r_{i}, e_{i}\right)=1$. Also, fix maximal tori $T_{i}$ such that $T_{i}(\mathfrak{o}) \subset P_{i}$.

It is immediate from Lemma 3.13 that the diagonal action of $\mathrm{GL}_{n}(\mathbb{C})$ on $\prod_{i \in I} \widetilde{\mathcal{M}}\left(P_{i}, r_{i}\right)$ is free canonical Poisson and that its moment map $\mu=\sum_{i \in I}$ res $_{i}$ is a submersion.

Definition 3.14. Define $\widetilde{\mathcal{M}}(\mathbf{x}, \mathbf{P}, \mathbf{r})$ as the Poisson reduction

$$
\widetilde{\mathcal{M}}(\mathbf{x}, \mathbf{P}, \mathbf{r})=\prod_{i \in I} \widetilde{\mathcal{M}}\left(P_{i}, r_{i}\right) / / 0 \mathrm{GL}_{n}(\mathbb{C}) .
$$

We also set $\widehat{\mathcal{M}}(\mathbf{x}, \mathbf{P}, \mathbf{r})=\mu^{-1}(0)$ and $\mathcal{A}(\mathbf{x}, \mathbf{P}, \mathbf{r})=\prod_{i \in I} \mathcal{A}\left(P_{i}, r_{i}\right)$.

Proposition 3.15. There is a smooth map $\mathbf{\Gamma}: \widetilde{\mathcal{M}}(\mathbf{x}, \mathbf{P}, \mathbf{r}) \rightarrow \mathcal{A}(\mathbf{x}, \mathbf{P}, \mathbf{r})$ which assigns a normalized formal type $\tilde{A}_{i}$ to $\nabla$ at each pole $x_{i}$. The fiber $\boldsymbol{\Gamma}^{-1}(\tilde{\mathbf{A}})$ is isomorphic to $\widetilde{\mathcal{M}}(\mathbf{A})$, and the symplectic leaves of $\widetilde{\mathcal{M}}(\mathbf{x}, \mathbf{P}, \mathbf{r})$ are the connected components of these fibers.

Proof. Since the maps $\Gamma_{i}: \widetilde{\mathcal{M}}\left(P_{i}, r_{i}\right) \rightarrow \mathcal{A}\left(P_{i}, r_{i}\right)$ are $\mathrm{GL}_{n}(\mathbb{C})$-equivariant (where $\mathrm{GL}_{n}(\mathbb{C})$ acts trivially on $\left.\mathcal{A}\left(P_{i}, r_{i}\right)\right), \prod_{i \in I} \Gamma_{i}$ induces the desired map $\boldsymbol{\Gamma}$. The statement about the fibers of $\boldsymbol{\Gamma}$ follows from Proposition 3.9 and the construction of $\widetilde{\mathcal{M}}(\mathbf{A})$ in Theorem 3.6. Finally, the symplectic leaves of $\prod_{i} \widetilde{\mathcal{M}}\left(P_{i}, r_{i}\right)$ are given by $\prod_{i} \widetilde{\mathcal{M}}\left(A_{i}\right)$, and they intersect the $\mathrm{GL}_{n}(\mathbb{C})$-orbits cleanly. By Proposition 3.10, the symplectic leaves of $\widetilde{\mathcal{M}}(\mathbf{x}, \mathbf{P}, \mathbf{r})$ are the connected components of $\widetilde{\mathcal{M}}(\mathbf{A})$.

Theorem 3.16. The Poisson manifold $\widetilde{\mathcal{M}}(\mathbf{x}, \mathbf{P}, \mathbf{r})$ is isomorphic to the moduli space of isomorphism classes of triples $(V, \nabla, \mathbf{g})$, where $(V, \nabla, \mathbf{g})$ satisfies the first two conditions of Definition [3.2, and $\left(V_{i}, \nabla_{i}\right)$ contains a regular stratum of the form $\left(P_{i}, r_{i}, \beta\right)$. The manifold $\widehat{\mathcal{M}}(\mathbf{x}, \mathbf{P}, \mathbf{r})$ is isomorphic to the moduli space of isomorphism classes $(V, \nabla, \mathbf{g})$ satisfying the conditions above, with a fixed global trivialization $\phi$.

Proof. By Proposition 2.9, we may associate a unique formal type $A$ to every formal connection that contains a regular stratum $(P, r, \beta)$. Therefore, if $(V, \nabla, \mathbf{g})$ satisfies the conditions above, there is a unique element $\mathbf{A} \in \mathcal{A}\left(P_{i}, r_{i}\right)$ given by the formal type of $(V, \nabla, \mathbf{g})$ at each singular point. In particular, by Theorem 3.6, $(V, \nabla, \mathbf{g})$ corresponds to a unique point in $\widetilde{\mathcal{M}}(\mathbf{A})$. However, by Proposition 3.15. $\widetilde{\mathcal{M}}(\mathbf{A}) \cong$ 
$\boldsymbol{\Gamma}^{-1}(\mathbf{A})$. On the other hand, every point $p \in \widetilde{\mathcal{M}}(\mathbf{x}, \mathbf{P}, \mathbf{r})$ corresponds to a unique connection with formal type $\boldsymbol{\Gamma}(p)$.

Now, suppose $m=\left(U_{i} g_{i}, \alpha_{i}\right)_{i \in I} \in \widehat{\mathcal{M}}(\mathbf{x}, \mathbf{P}, \mathbf{r})$. Fix a global form $\nu \in \Omega_{\mathbb{p} 1}^{1}$. By (2.1), we may associate to $\alpha_{i}$ a unique meromorphic form $\alpha_{i \nu} \nu$ with coefficients in $\mathfrak{g l}_{n}\left(F_{i}\right)$. Since $\mu(m)=0, \sum_{i \in I} \operatorname{Res}_{i}\left(\alpha_{i \nu} \nu\right)=0$. It follows that $q$ determines a global form $\alpha \nu$, and thus a global meromorphic connection $\nabla=d+\alpha \nu$ on the trivial rank $n$ vector bundle over $\mathbb{P}^{1}$. It is easily checked that this gives a bijection between points in $\widehat{\mathcal{M}}(\mathbf{x}, \mathbf{P}, \mathbf{r})$ and triples $(V, \nabla, \mathbf{g})$.

\section{INTEGRABLE DEFORMATIONS}

Let $X=\mathbb{P}^{1}(\mathbb{C})$, and let $V$ be an $n$-dimensional trivial vector bundle on $X$. In this section, we will consider the deformations of a connection $(V, \nabla)$ which contains a regular stratum at each singularity.

4.1. Formal Deformations. Without loss of generality, take $x_{1}=0$ and fix a parameter $z$ at 0 . We will suppress the subscripts on $\left(P_{i}, r_{i}, \beta_{i}\right)$, etc. when we work locally at 0 . Let $F$ be the field of Laurent series at 0 and $\mathfrak{o} \subset F$ the ring of power series. Define $\hat{V}$ to be the formal completion of $V$ at 0 , and let $\hat{\nabla}$ be the induced formal connection.

Now, let $D=\operatorname{Spec}(\mathfrak{o})$, and fix a standard parahoric $P$ and an integer $r$ with $\operatorname{gcd}(r, e)=1$. We also fix a torus $T$, with $T(\mathfrak{o}) \subset P$, as in (2.2). Let $\Delta$ be an analytic polydisk; we denote its ring of functions by $R$. A formal flat deformation is a flat, meromorphic connection $(\bar{V}, \bar{\nabla})$ on $D \times \Delta$ satisfying the following properties:

- the vector bundle $\bar{V}$ is isomorphic to the trivial rank $n$ vector bundle, and

- the restriction of $\bar{\nabla}$ to $D \times\{y\}$, denoted by $\hat{\nabla}_{y}$, contains a regular stratum $\left(P_{y}, r, \beta_{y}\right)$.

Fix a trivialization $\phi$ of $\bar{V}$ so that we may identify all other trivializations with elements of $\mathrm{GL}_{n}(\mathfrak{o} \otimes R)$. We say that the deformation $(\bar{V}, \bar{\nabla})$ is framed if there exists a trivialization $g \in \mathrm{GL}_{n}(R)$ with the property that $P_{y}=P^{g(y)}, \beta_{y}=\beta^{g(y)}$, and the regular stratum $\left(P_{y}, r, \beta_{y}\right)$ is centralized by $T_{y}:=T^{g(y)}$. In particular, any representative $\left(\beta_{y}\right)_{\nu} \in \mathfrak{P}_{y}^{-r}$ for $\beta_{y}$ lies in $\mathfrak{t}_{y}+\mathfrak{P}_{y}^{-r+1}$ by [2, Remark 3.5] (with $\operatorname{ord}(\nu)=-1)$.

We denote $\mathfrak{P}_{\Delta}^{\ell}=\mathfrak{P}^{\ell} \otimes R, \overline{\mathfrak{P}}_{\Delta}^{\ell}=\mathfrak{P}_{\Delta}^{\ell} / \mathfrak{P}_{\Delta}^{\ell+1}$, and $\mathfrak{t}_{\Delta}=\mathfrak{t} \otimes R$. We define $P_{\Delta}$ and $T_{\Delta}$ similarly. Suppose that $(\bar{V}, \vec{\nabla})$ is framed by $g$, and fix a nonzero one-form $\nu$ at 0 . If $\nu=u \frac{d z}{z}$, write $\frac{z \nu}{d z}=u$. Let $A(y)$ be the formal type of $\bar{\nabla}$ at $y$. Using the pairing in (2.1), we may choose a representative $A_{\nu}(y)$ for $A(y)$ of the form

$$
A_{\nu}(y)=\left(\frac{-r}{n} t_{-r}(y) \varpi_{T}^{-r}+\ldots+\frac{-1}{n} t_{-1}(y) \varpi_{T}^{-1}+t_{0}(y)\right) \frac{d z}{z \nu},
$$

with $t_{i}(y) \in \mathfrak{t}_{\Delta}^{b}$. For example, $A_{\frac{d z}{z}}=\left(\frac{-r}{n} t_{-r}(y) \varpi_{T}^{-r}+\ldots+\frac{-1}{n} t_{-1}(y) \varpi_{T}^{-1}+t_{0}(y)\right)$.

Recall that any element of $\mathfrak{t}$ can be written as a Laurent series $t=\sum_{i=-N}^{\infty} t_{j} \varpi_{T}^{j}$ with $t_{i} \in \mathfrak{t}^{b}$. We define an endomorphism $\delta_{e}$ of $\mathfrak{t}$ via $\delta_{e}(t)=\sum_{i=-N}^{\infty} \frac{i}{e} t_{i} \varpi_{T}^{i}$.

Lemma 4.1. Suppose $t \in \mathfrak{t}_{\Delta}$. Then, $z \partial_{z} t-\left[t, H_{T}\right]=\delta_{e} t$. Moreover, any solution $B \in \Omega_{\Delta}^{1}\left(\left(\mathfrak{t}+\mathfrak{P}^{\ell}\right) / \mathfrak{P}^{\ell}\right)$ to the differential equation $z \partial_{z} B-\left[B, H_{T}\right]=\delta_{e} t+t_{0}+\mathfrak{P}^{\ell}$ has the form $B=t+f+\mathfrak{P}^{\ell}$, where $f \in \mathfrak{t}_{\Delta}^{b}$. There is no solution when $\ell \geq 0$ and $t_{0} \neq 0$. 
Proof. Since the equations are block diagonal in $\mathfrak{t}$, we may immediately reduce to the case where $T=E^{\times}$. A direct calculation shows that $z \partial_{z} \varpi_{T}^{i}-\left[\varpi_{T}, H_{E}\right]=$ $\frac{i}{e} \varpi_{T}^{i}=\delta_{e}\left(\varpi_{T}^{i}\right)$. This proves the first statement. The second follows by applying the same calculation to each term of $B$ up to $\varpi_{T}^{\ell}$. Note that $z \partial_{z} \varpi_{T}^{0}-\left[\varpi_{T}^{0}, H_{E}\right]=0$, so there is no solution when $t_{0} \neq 0$.

As in the previous section, we will let $p \cdot[\bar{\nabla}]_{g}$ denote the $d_{z}$ part of the gauge transformation formula: $p \cdot[\bar{\nabla}]_{g}=\operatorname{Ad}(p)\left([\bar{\nabla}]_{g}\right)-\left(d_{z} p\right) p^{-1}$. Similarly, upon fixing $\nu \in \Omega_{F}^{1}(R)$, we write $p \cdot M=\operatorname{Ad}(p)(M)-\left(\tau_{\nu} p\right) p^{-1}$ when $M \in \mathfrak{g l}_{n}\left(F \otimes_{\mathbb{C}} R\right)$. We will use $d_{\Delta}$ and $\bar{d}$ to denote the exterior differential on $\Delta$ and $\mathbb{P}^{1} \times \Delta$, respectively. Suppose that $\tilde{A}_{\nu}$ is the normalized formal type associated to $A_{\nu}$. By Proposition 2.11, there exists an element $p \in P_{\Delta}$ such that

$$
p(y) \cdot\left[\hat{\nabla}_{y}\right]_{g}=\tilde{A}_{\nu}(y) \nu .
$$

Let $d_{\Delta}$ be the exterior differential on $\Delta$. Define

$$
A_{\Delta}(y)=\sum_{i=-r}^{-1} \varpi_{T}^{-i} d_{\Delta} t_{i}
$$

so that $d_{\Delta} A_{\frac{d z}{z}}=\delta_{e} A_{\Delta}+d_{\Delta} t_{0}$.

Proposition 4.2. Let $p$ be as above. Then, $p \cdot[\bar{\nabla}]_{g}-\left(d_{\Delta} p\right) p^{-1}=\tilde{A}_{\nu} \nu+A_{\Delta}+f$, where $f \in \Omega_{\Delta}^{1}\left(\mathfrak{t}^{\mathfrak{b}}\right)$ is closed. Moreover, $t_{0}(y)$ must be constant. Conversely, if $q \in$ $\mathrm{GL}_{n}(F \otimes R), f$ is closed, and $t_{0}(y)$ is constant, then $\left[\bar{\nabla}^{\prime}\right]=q^{-1} \cdot\left(\tilde{A}_{\nu} \nu+A_{\Delta}+f\right)+$ $q^{-1}\left(d_{\Delta} q\right)$ determines a flat meromorphic connection $\bar{\nabla}^{\prime}$ on $\bar{V}$.

Remark 4.3. The case $e=1$ is proved in the Appendix of [1].

Proof. Note that the connection determined by $p \cdot[\bar{\nabla}]_{g}-\left(d_{\Delta} p\right) p$ is flat, since $\bar{\nabla}$ is flat and $-\left(d_{\Delta} p\right) p$ is simply the $d_{\Delta}$ part of the gauge transformation formula. Conversely, if the connection determined by $\tilde{A}_{\nu} \nu+A_{\Delta}+f$ is flat, then $\bar{\nabla}^{\prime}$ is flat by the same argument.

Without loss of generality, set $\nu=\frac{d z}{z}$. It suffices to show that whenever $[\bar{\nabla}]=$ $\tilde{A}_{\frac{d z}{z}} \frac{d z}{z}+B$ for some $B \in \Omega_{\Delta}^{1}\left(\mathfrak{g l}_{n}(F)\right)$, then $\bar{\nabla}$ is flat if and only if $B$ has the form $A_{\Delta}+f$. We observe that $\bar{\nabla}$ is flat if and only if it satisfies the conditions

$$
\begin{gathered}
d_{\Delta}\left(A_{\frac{d z}{z}}\right)-z \partial_{z} B+\left[B, A_{\frac{d z}{z}}+H_{T}\right]=0 \\
\text { and } d_{\Delta} B+B \wedge B=0 .
\end{gathered}
$$

If $B=A_{\Delta}+f$, (4.2) holds trivially while (4.1) follows from the first part of Lemma 4.1] so $\bar{\nabla}$ is flat.

We now prove the converse. If $r=0$, then $e=1$ and $H_{T}=0$. In this case, we may take $A_{\frac{d z}{z}}=t_{0}(y)$ to be a regular diagonal matrix with entries in $R$; moreover, no two eigenvalues of $A_{\frac{d z}{z}}$ differ by an integer. Setting $B=\sum B_{\ell} z^{\ell}$ with $B_{\ell} \in$ $\mathfrak{g l}_{n}(\mathbb{C})$, (4.2) reduces to $\left[B_{\ell}, A_{\frac{d z}{z}}\right]=\ell B_{\ell}$ when $\ell \neq 0$. The eigenvalue condition now implies that $B_{\ell}=0$. whenever $\ell \neq 0$, by the condition on the eigenvalues of $A_{\frac{d z}{z}}$. On the other hand, $\left[B_{0}, A_{\frac{d z}{z}}\right]=-d_{\Delta}\left(A_{\frac{d z}{z}}\right)$. Since the right hand side is a diagonal matrix, both sides must be identically 0 . It follows that $d_{\Delta}\left(A_{\frac{d z}{z}}\right)=0$ and $B_{0} \in \Omega_{\Delta}^{1}\left(\mathfrak{t}^{\mathrm{b}}\right)$ while the fact that $B_{0}$ is closed follows from (4.2). 
We now consider the case $r \geq 1$. In the following, let $t_{0}(y)$ be the constant term of $A_{\frac{d z}{z}}$. Suppose, by induction, that $B \in A_{\Delta}+f+\Omega_{\Delta}^{1}\left(\mathfrak{t}^{\mathfrak{b}}+\mathfrak{P}^{\ell}\right)$. (Note that $B \in \Omega^{1}\left(\mathfrak{P}^{L}\right)$ for some $L \leq-r$, so the first inductive step is trivially satisfied for $\ell=L)$. Applying the first part of Lemma 4.1 with $t=A_{\Delta}$ gives

$$
d_{\Delta}\left(A_{\frac{d z}{z}}\right)+\left[B, H_{T}\right]-z \partial_{z} B \in d_{\Delta} t_{0}+\Omega_{\Delta}^{1}\left(\mathfrak{P}^{\ell}\right) .
$$

We deduce from (4.1) that $\left[B, A_{\frac{d z}{z}}\right] \in d_{\Delta} t_{0}+\Omega_{\Delta}^{1}\left(\mathfrak{P}^{\ell}\right)$.

When $\ell<1, d_{\Delta} t_{0} \in \Omega_{\Delta}^{1}\left(\mathfrak{P}^{\ell}\right)$. This implies that $\left[B, A_{\frac{d z}{z}}\right] \in \Omega_{\Delta}^{1}\left(\mathfrak{P}^{\ell}\right)$, so $B \in$ $\Omega_{\Delta}^{1}\left(\mathfrak{t}+\mathfrak{P}^{\ell+r}\right)$. Next, consider $\ell=1$. By the $\ell=0$ step, we know that $B \in \Omega_{\Delta}^{1}(\mathfrak{t}+$ $\left.\mathfrak{P}^{r}\right)$. Part (3) of Proposition 2.4 shows that $\left[B, A_{\frac{d z}{z}}\right]+\Omega_{\Delta}^{1}\left(\mathfrak{P}^{1}\right) \in \operatorname{ker}\left(\bar{\pi}_{\mathfrak{t}} \otimes \mathrm{I}_{\Omega_{\Delta}^{1}}\right)$. Since $d_{\Delta} t_{0} \in \Omega_{\Delta}^{1}\left(\mathfrak{t}^{b}\right)$, part (1) of the same proposition gives $d_{\Delta} t_{0} \in \Omega_{\Delta}^{1}\left(\mathfrak{P}^{1}\right)$. Since $\mathfrak{t}^{b} \cap \mathfrak{P}^{1}=\{0\}$, we see that $d_{\Delta} t_{0}=0$. Thus, we may conclude that the inductive hypothesis implies that $\left[B, A_{\frac{d z}{z}}\right] \in \Omega_{\Delta}^{1}\left(\mathfrak{P}^{\ell}\right)$ for $\ell \geq 1$ as well. As before, $B \in$ $\Omega_{\Delta}^{1}\left(\mathfrak{t}+\mathfrak{P}^{\ell+r}\right) \subset \Omega_{\Delta}^{1}\left(\mathfrak{t}+\mathfrak{P}^{\ell+1}\right)$.

To complete the inductive step, we apply the second part of Lemma 4.1 to obtain $B \in A_{\Delta}+\Omega_{\Delta}^{1}\left(\mathfrak{t}^{\mathfrak{b}}+\mathfrak{P}^{\ell+1}\right)$. Using the fact that the sum $\mathfrak{t}^{b}+\mathfrak{P}^{\ell}$ is direct for $\ell \geq 1$, we actually obtain $f \in \Omega_{\Delta}^{1}\left(\mathfrak{t}^{b}\right)$ such that $B \in A_{\Delta}+f+\Omega_{\Delta}^{1}\left(\mathfrak{P}^{\ell}\right)$ for all $\ell$. The result now follows since $\bigcap_{\ell} \mathfrak{P}^{\ell}=0$.

Definition 4.4. We say that a compatible framing $g$ for $\bar{\nabla}$ is good if there exists $p \in P_{\Delta}^{1}$ such that $p \cdot[\bar{\nabla}]_{g}-\left(d_{\Delta} p\right) p^{-1}=\tilde{A}_{\nu} \nu+A_{\Delta}$.

Proposition 4.5. Every framed flat deformation has a good compatible framing.

Proof. If $g$ is a compatible framing, there exists $p \in P$ such that $p \cdot\left([\bar{\nabla}]_{g}\right)-$ $\left(d_{\Delta} p\right) p^{-1}=\left(A_{\frac{d z}{z}}+H_{T}\right) \frac{d z}{z}+A_{\Delta}+f$ by Proposition 4.2. Since $f$ is closed on $\Delta$, it is exact. Choosing $\varphi \in \mathfrak{t}_{\Delta}^{b}$ such that $f=d_{\Delta} \varphi$, we obtain

$$
\operatorname{Ad}\left(e^{\varphi}\right)\left(p \cdot\left([\bar{\nabla}]_{g}\right)-d_{\Delta}(p) p^{-1}\right)-d_{\Delta} \varphi=\left(A_{\frac{d z}{z}}+H_{T}\right) \frac{d z}{z}+A_{\Delta} .
$$

It follows that $e^{\varphi} g$ is a good compatible framing.

\subsection{Global Deformations.}

Definition 4.6. A framed global deformation is a triple $(\mathbf{g}, \bar{V}, \tilde{\nabla})$ consisting of:

(1) a trivializable rank $n$ vector bundle $\bar{V}$ on $\mathbb{P}^{1} \times \Delta$;

(2) an $R$-relative connection $\tilde{\nabla}$;

(3) a collection of analytic framings $\mathbf{g}=\left(g_{i}\right)_{i \in I}, g_{i}: \Delta \rightarrow U_{i} \backslash \mathrm{GL}_{n}(\mathbb{C})$;

(4) the restriction of $(\bar{V}, \tilde{\nabla})$ to $\mathbb{P}^{1} \times\{y\}$ must lie in $\widetilde{\mathcal{M}}(\mathbf{x}, \mathbf{P}, \mathbf{r})$ with compatible framing $\mathbf{g}(y)$.

We say that a framed deformation is integrable if there exists a flat $\mathbb{C}$-relative connection $\bar{\nabla}$ on $\mathbb{P}^{1} \times \Delta$ with $\mathbb{P}^{1}$ part $\tilde{\nabla}$.

We note that $(\mathbf{g}, \bar{V}, \tilde{\nabla})$ determines a smooth map $\Delta \rightarrow \widetilde{\mathcal{M}}(\mathbf{x}, \mathbf{P}, \mathbf{r})$. Specifically, there are maps $g_{i}(y)$ and $\alpha_{i}(y)$ such that the connection on the fiber above $y$ corresponds to the point $\left(U_{i} g_{i}(y), \alpha_{i}(y)\right)_{i \in I} \in \widetilde{\mathcal{M}}(\mathbf{x}, \mathbf{P}, \mathbf{r})$.

Suppose that $(\mathbf{g}, \bar{V}, \tilde{\nabla})$ is an integrable framed global deformation. If we fix a trivialization for $\bar{V}$, we may write $[\bar{\nabla}]=\alpha \nu+\Upsilon$, where $\Upsilon$ is a section of 
$\Omega_{\Delta / \mathbb{C}}^{1}(\operatorname{End}(\bar{V}))$ with poles along $\left\{x_{i}\right\}$. The curvature of $\bar{\nabla}$ is given by

$$
\Xi(\alpha, \Upsilon)=\bar{d}(\alpha \nu+\Upsilon)+\Upsilon \wedge \alpha \nu+\alpha \nu \wedge \Upsilon+\Upsilon \wedge \Upsilon \in \Omega_{\Delta \times \mathbb{P}^{1}}^{2}
$$

Thus, $\bar{\nabla}$ is flat if and only if the following hold:

$$
\tau_{\nu} \Upsilon=d_{\Delta} \alpha+[\Upsilon, \alpha] \quad \text { and } \quad 0=d_{\Delta} \Upsilon+\Upsilon \wedge \Upsilon
$$

An integrable deformation $(\mathbf{g}, \bar{V}, \tilde{\nabla})$ determines a flat formal deformation $\left(\hat{V}_{i}, \bar{\nabla}_{i}\right)$ at each singular point. Therefore, if $\tilde{A}_{i}(y)$ is the normalized formal type of $\left(\hat{V}_{i}, \bar{\nabla}_{i}\right)$ at $y \in \Delta$, Proposition 4.5 implies that there exists $p_{i} \in P_{i}^{1}$ such that $p_{i} g_{i} \cdot[\bar{\nabla}]-$ $\operatorname{Ad}\left(p_{i}\right)\left(d_{\Delta} g_{i} g_{i}^{-1}\right)-\left(d_{\Delta} p_{i}\right) p_{i}^{-1}=\tilde{A}_{i, \nu} \nu+A_{i, \Delta}$. Since $p_{i}^{-1} d_{\Delta} p_{i} \in \Omega_{\Delta}^{1}\left(\mathfrak{P}^{1}\right)$ by 2 , Lemma 4.4], we deduce

$$
\Upsilon \in \operatorname{Ad}\left(g_{i}^{-1} p_{i}^{-1}\right) A_{i, \Delta}+g_{i}^{-1} d_{\Delta} g_{i}+\Omega_{\Delta}^{1}\left(\left(\mathfrak{P}_{i}^{1}\right)^{g_{i}}\right) .
$$

Set $\mathbf{r}_{\mathbf{e}}=\left(r_{i}^{\prime}\right)_{i \in I}:=\left(\left\lceil\frac{r_{i}}{e_{i}}\right\rceil\right)_{i \in I}$, where $\left\lceil\frac{r_{i}}{e_{i}}\right\rceil$ is the integer ceiling of $\frac{r_{i}}{e_{i}}$. Let $D_{\mathbf{r}}$ be the divisor $\sum_{i \in I} r_{i}^{\prime}\left[x_{i}\right]$ on $\mathbb{P}^{1}$.

Definition 4.7. Let $\mathfrak{g l}_{n \mathbb{P}^{1}}$ denote the trivial $\mathfrak{g l}_{n}$-bundle on $\mathbb{P}^{1}$, and let $\mathfrak{g r}_{n \mathbb{P}^{1}}^{\mathrm{r}_{\mathrm{e}}}=$ $\mathfrak{g l}_{n \mathbb{P}^{1}}\left(D_{\mathbf{r}}\right)$ be the sheaf corresponding to the divisor $D_{\mathbf{r}}$

Note that sections of $\mathfrak{g l}_{n \mathbb{P}^{1}}^{\mathbf{r}_{\mathbf{e}}}$ have poles of order at most $r_{i}^{\prime}$ at $x_{i}$.

Fix a set of parameters $\left(z_{i}\right)_{i \in I}$ at each singular point $x_{i}$ with the property that each $z_{i}$ has a pole at a fixed point $x_{0}$. Define a $\mathbb{C}$-linear map $\phi_{i}: \mathfrak{g l}_{n}\left(F_{i}\right) \rightarrow \mathfrak{g l}_{n}(\mathbb{C})$ by

$$
\phi_{i}(X)=\operatorname{Res}_{x_{i}}\left(X \frac{d z_{i}}{z_{i}}\right) .
$$

Thus, $\phi_{i}$ extracts the constant term of $X$ at $x_{i}$ with respect to $z_{i}$. This induces a $\operatorname{map} \bar{\phi}_{i}: \mathfrak{g l}_{n}\left(F_{i}\right) / \mathfrak{P}_{i}^{1} \rightarrow \mathfrak{g l}_{n}(\mathbb{C}) / \mathfrak{u}_{i}$.

Next, we define a map $\varsigma$ which assigns a global section of $\mathfrak{g l}_{n \mathbb{P}^{1}}^{\mathrm{r}_{\mathrm{e}}}$ to a collection of principal parts at $\mathbf{x}$. Given $X_{i} \in \mathfrak{g}_{i}^{-r_{i}^{\prime}} / \mathfrak{g}_{i}$, let $\tilde{X}_{i} \in H^{0}\left(\mathbb{P}^{1} ; \mathfrak{g l}_{n \mathbb{P}^{1}}^{r_{e}}\right)$ be the section corresponding to the unique lift of $X_{i}$ to $\mathfrak{g l}_{n}\left(z_{i}^{-1} \mathbb{C}\left[z_{i}^{-1}\right]\right)$.

Definition 4.8. The map $\varsigma: \prod_{i \in I} \mathfrak{g}_{i}^{-r_{i}^{\prime}} / \mathfrak{g}_{i} \rightarrow H^{0}\left(\mathbb{P}^{1} ; \mathfrak{g}_{n \mathbb{P}^{1}}^{\mathbf{r}_{\mathbf{e}}}\right)$ is given by

$$
\varsigma\left(\left(X_{i}\right)_{i \in I}\right)=\sum_{i \in I} \tilde{X}_{i} .
$$

We will usually write $X^{0}$ for $\varsigma\left(\left(X_{i}\right)_{i \in I}\right)$.

Remark 4.9. This map commutes with the adjoint action of $\mathrm{GL}_{n}(\mathbb{C})$, i.e., $\operatorname{Ad}(g)\left(X^{0}\right)=$ $(\operatorname{Ad}(g) X)^{0}$ for any $g \in \mathrm{GL}_{n}(\mathbb{C})$. Indeed, $\operatorname{Ad}(g)\left(\tilde{X}_{i}\right)=\widetilde{\operatorname{Ad}(g) X_{i}}$ for each $i$ since $\operatorname{Ad}(g)$ stabilizes $\mathfrak{g l}_{n}\left(z_{i}^{-1} \mathbb{C}\left[z_{i}^{-1}\right]\right)$.

We are now ready to describe a system of differential equations satisfied by an integrable deformation. Let $(\bar{V}, \bar{\nabla})$ be a deformation of $(V, \nabla)$ as in Definition 4.6, corresponding to a map $\left(g_{i}(y), \alpha_{i}(y)\right)_{i \in I}$ from $\Delta$ to $\widetilde{\mathcal{M}}(\mathbf{x}, \mathbf{P}, \mathbf{r})$.

Fix a uniformizer $z_{0}$ at $x_{0}$.

Lemma 4.10. If $\Upsilon^{\prime}$ has principal part $\Upsilon_{i}$ at each $x_{i}$ and is holomorphic elsewhere, then $\Upsilon^{\prime}-\Upsilon^{0}=\left.\Upsilon^{\prime}\right|_{z_{0}=0} \in \Omega_{\Delta}^{1}\left(\mathfrak{g l}_{n}(\mathbb{C})\right)$. Here, $\Upsilon^{0}=\varsigma\left(\left(\Upsilon_{i}\right)_{i \in I}\right)$ as above. 
Proof. Since $\Upsilon^{\prime}$ and $\Upsilon^{0}$ have the same principal parts at each singular point, $\Upsilon^{\prime}-$ $\Upsilon^{0}=X \in \Omega_{\Delta}^{1}\left(\mathfrak{g l}_{n}(\mathbb{C})\right)$. By construction, $\Upsilon^{0}$ and $\Upsilon^{\prime}$ are holomorphic at $x_{0}$, and $\left.\Upsilon^{0}\right|_{z_{0}=0}=0$. Therefore, $X=\left.\Upsilon^{\prime}\right|_{z_{0}=0}$.

Lemma 4.11. Given $\Upsilon \in \Omega_{\Delta}^{1}\left(H^{0}\left(\mathbb{P}^{1} ; \mathfrak{g}_{n \mathbb{P}^{1}}^{\mathbf{r}_{\mathbf{e}}}\right)\right)$, define $\Upsilon_{i}=\Upsilon+\mathfrak{g}_{i}$. Any integrable framed deformation $\bar{\nabla}$ is $G L_{n}(R)$-gauge equivalent to a deformation $\alpha \nu+\Upsilon$ satisfying $\Upsilon=\varsigma\left(\left(\Upsilon_{i}\right)_{i \in I}\right)$.

Proof. Suppose that $[\bar{\nabla}]=\alpha^{\prime} \nu+\Upsilon^{\prime}$. Then, $\Upsilon^{\prime}-\left(\Upsilon^{\prime}\right)^{0}=X \in \Omega_{\Delta}^{1}\left(\mathfrak{g l}_{n}(\mathbb{C})\right)$ by Lemma 4.10. Since the image of $\varsigma$ is closed under conjugating by $\mathrm{GL}_{n}(R)$, it suffices to show that there exists $g \in \mathrm{GL}_{n}(R)$ such that $\Upsilon:=\operatorname{Ad}(g) \Upsilon^{\prime}-\left(d_{\Delta} g\right) g^{-1}=$ $\operatorname{Ad}(g)\left(\Upsilon^{\prime}\right)^{0}$.

The system of differential equations $g^{-1}\left(d_{\Delta} g\right)=X$ has a solution if $d_{\Delta} X+X \wedge$ $X=0$. To see this, recall that $d_{\Delta} X=\left.d_{\Delta} \Upsilon^{\prime}\right|_{z_{0}=0}$. By flatness, $d_{\Delta} \Upsilon^{\prime}+\Upsilon^{\prime} \wedge \Upsilon^{\prime}=0$, so $d_{\Delta} X+X \wedge X=\left.\left(d_{\Delta} \Upsilon^{\prime}+\Upsilon^{\prime} \wedge \Upsilon^{\prime}\right)\right|_{z_{0}=0}=0$.

Now, suppose that $(\mathbf{g}, \bar{V}, \tilde{\nabla})$ is a deformation. Choose $p_{i} \in P_{\Delta}^{1}$ such that $p_{i} \cdot \alpha \nu=$ $\tilde{A}_{i \nu} \nu$, and write $\hat{g}_{i}=p_{i} g_{i}$. Let $\Upsilon_{i}=\operatorname{Ad}\left(\hat{g}_{i}^{-1}\right) A_{i, \Delta}+\Omega_{\Delta}^{1}\left(\mathfrak{g}_{i}\right)$, with corresponding global section $\Upsilon^{0} \in \Omega_{\Delta}^{1}\left(H^{0}\left(\mathbb{P}^{1} ; \mathfrak{g r}_{n \mathbb{P}^{1}}^{\mathrm{r}_{\mathrm{e}}}\right)\right)$.

Theorem 4.12. A good framed deformation $(\mathrm{g}, \bar{V}, \tilde{\nabla})$ is integrable if and only if it is $\mathrm{GL}_{n}(R)$-gauge-equivalent to a deformation satisfying the following equations:

(1) $\phi_{i}\left(\operatorname{Ad}\left(p_{i}^{-1}\right)\left(A_{i, \Delta}\right)+\left(d_{\Delta} g_{i}\right) g_{i}^{-1}\right) \in \phi_{i}\left(\operatorname{Ad}\left(g_{i}\right) \Upsilon^{0}\right)+\Omega_{\Delta}^{1}\left(\mathfrak{u}_{i}\right)$;

(2) $d_{\Delta} \alpha \in \tau_{\nu} \Upsilon^{0}+\left[\alpha, \Upsilon^{0}\right]+\Omega_{\Delta}^{1}\left(\mathfrak{g}_{i} \frac{d z_{i}}{z_{i} \nu}\right)$; and

(3) $d \Upsilon^{0}+\Upsilon^{0} \wedge \Upsilon^{0}=0$.

Proof. Suppose that $(\mathrm{g}, \bar{V}, \tilde{\nabla})$ is integrable. By Lemma 4.11, there exists a gauge $g \in \mathrm{GL}_{n}(R)$ such that $[\bar{\nabla}]_{g}=\alpha \nu+\Upsilon$ with $\Upsilon=\Upsilon^{0}$. By Proposition 4.2.

$$
\Upsilon \in \operatorname{Ad}\left(\hat{g}_{i}^{-1}\right) A_{i, \Delta}+\hat{g}_{i}^{-1} d_{\Delta} \hat{g}_{i}+\left(\mathfrak{P}_{i, \Delta}^{1}\right)^{g_{i}} .
$$

Therefore, condition (11) is satisfied by applying $\operatorname{Ad}\left(g_{i}\right)$ and $\phi_{i}$. Moreover, $\alpha \nu+\Upsilon$ is flat, so conditions (2) and (3) follow from (4.3).

To see the converse, note that condition (11) shows that $\Upsilon^{0}$ satisfies (4.7). Condition (2) implies that the cross-term of the curvature of $\alpha \nu+\Upsilon^{0}$ is zero modulo $\mathfrak{g}_{i}^{1} \frac{d z_{i}}{z_{i} \nu}$. Writing this term as $\sum f_{j} d y_{j}$, where the $d y_{j}$ 's are a basis for $\Omega_{\Delta}^{1}$, we see that $f_{j} \in H^{0}\left(\mathbb{P}^{1} ; \Omega_{\mathbb{P}^{1}}^{1}\right)=\{0\}$. Since the term in $\Omega_{\Delta}^{2}$ vanishes by condition (3), we see that the curvature vanishes.

Corollary 4.13. A good framed deformation $(\mathrm{g}, \bar{V}, \tilde{\nabla})$ is integrable if and only if there exists $g \in \mathrm{GL}_{n}(R)$ such that

(1) $\phi_{i}\left(\operatorname{Ad}\left(g_{i}\right)\left(g^{-1} d_{\Delta} g+\Upsilon^{0}\right)\right) \in \phi_{i}\left(\operatorname{Ad}\left(p_{i}^{-1}\right) A_{\Delta, i}+\left(d_{\Delta} g_{i}\right) g_{i}^{-1}\right)+\Omega_{\Delta}^{1}\left(\mathfrak{u}_{i}\right)$;

(2) $d_{\Delta} \alpha \in \tau_{\nu} \Upsilon^{0}+\left[\alpha, \Upsilon^{0}+g^{-1} d_{\Delta} g\right]+\Omega_{\Delta}^{1}\left(\mathfrak{g}_{i}^{1} \frac{d z_{i}}{z_{i}}\right)$; and

(3) $d_{\Delta}\left(\Upsilon^{0}+g^{-1} d_{\Delta} g\right)+\left(\Upsilon^{0}+g^{-1} d_{\Delta} g\right) \wedge\left(\Upsilon^{0}+g^{-1} d_{\Delta} g\right)=0$.

Proof. Set $[\bar{\nabla}]=\alpha \nu+\Upsilon$ and choose $g \in \mathrm{GL}_{n}(R)$ such that $[\bar{\nabla}]_{g}=\alpha^{\prime} \nu+\Upsilon^{\prime}$ with $\Upsilon^{\prime}=\left(\Upsilon^{\prime}\right)^{0}$. The proof above shows that $\left(\mathbf{g}^{\prime}, \alpha^{\prime}, \Upsilon^{\prime}\right)$ satisfy the conditions in the theorem. One obtains the equations in the corollary by substituting $\Upsilon^{\prime}=\operatorname{Ad}(g) \Upsilon^{0}$, $\alpha^{\prime}=\operatorname{Ad}(g)(\alpha)$, and $g_{i}^{\prime}=g_{i} g^{-1}$. The converse is proved similarly.

Remark 4.14. It will be shown later in Theorem 6.8 that the third condition in these two results is unnecessary. 


\section{The Differential Ideal}

In this section and the next, we show that the equations from Corollary 4.13 determine a Frobenius integrable system on $\widetilde{\mathcal{M}}(\mathbf{x}, \mathbf{P}, \mathbf{r})$. Throughout, we will fix a global meromorphic one-form $\nu$ on $\mathbb{P}^{1}(\mathbb{C})$. We will also use the convention that if $B$ is a vector bundle on a manifold $M$ and $N \subset M$ is open, then we will write $\Omega_{N}^{1}(B)$ for $\Omega_{N}^{1}\left(\left.B\right|_{N}\right)$. Furthermore, if $\sigma$ is a global section of $\Omega_{M}^{1}(B)$, we will abuse notation and let $\sigma$ also denote $\left.\sigma\right|_{N}$.

5.1. Generators. We will construct a differential ideal $\mathcal{I}$ on $\widehat{\mathcal{M}}(\mathbf{x}, \mathbf{P}, \mathbf{r})$ corresponding to the system of differential equations in Corollary 4.13. Throughout, we will simplify notation by suppressing $\mathbf{x}, \mathbf{P}$, and $\mathbf{r}$ in the notation and using $d$ for the exterior differential on all spaces whenever there is no risk of ambiguity. We will also write $\widetilde{\mathcal{M}}_{i}=\widetilde{\mathcal{M}}\left(P_{i}, r_{i}\right)$ and $\mathcal{A}_{i}=\mathcal{A}\left(P_{i}, r_{i}\right)$.

Set $\mathfrak{t}_{i}^{j}=\mathfrak{t}_{i} \cap \mathfrak{P}_{i}^{j}$ and $T_{i}^{j}=T_{i} \cap P_{i}^{j}$. First, we define maps $\hat{\Gamma}_{i}: \widehat{\mathcal{M}} \rightarrow \mathcal{A}_{i}$ and $\hat{\Gamma}_{i \nu}: \widehat{\mathcal{M}} \rightarrow\left(\mathfrak{t}_{i}^{-r_{i}}+H_{T}\right) \frac{d z_{i}}{z_{i} \nu}: \hat{\Gamma}_{i}\left(\left(U_{j} g_{j}, \alpha_{j}\right)_{i \in I}\right)=\Gamma_{i}\left(U_{i} g_{i}, \alpha_{i}\right)$ and $\hat{\Gamma}_{i \nu}\left(\left(U_{j} g_{j}, \alpha_{j}\right)\right)$ is the standard representative of $\Gamma_{i}\left(U_{i} g_{i}, \alpha_{i}\right)$ in $\mathfrak{P}_{i}^{-r} \frac{d z_{i}}{z_{i} \nu}$ with Laurent expansion $\left(\gamma_{-r} \varpi_{i}^{-r}+\ldots+\gamma_{0} \varpi_{i}^{0}+H_{T}\right) \frac{d z_{i}}{z_{i} \nu}$. We also write $\hat{\Gamma}_{i}^{0}$ for the coefficient of $\varpi_{i}^{0} \frac{d z_{i}}{z_{i} \nu}$ in $\hat{\Gamma}_{i \nu}$.

We identify the tangent bundle of $\mathcal{A}_{i} \subset\left(\mathfrak{t}_{i} / \mathfrak{t}_{i}^{r+1}\right)^{\vee}$ with $\mathcal{A}_{i} \times \mathfrak{t}_{i}^{-r_{i}} / \mathfrak{t}_{i}^{1}$ using the pairing $\langle,\rangle_{\frac{d z_{i}}{z_{i}}}$. The differential of the map $\hat{\Gamma}_{i}: \widehat{\mathcal{M}} \rightarrow \mathcal{A}_{i}$ determines a section $d \hat{\Gamma}_{i}$ of $\Omega_{\widehat{\mathcal{M}}}^{1}\left(\mathfrak{t}_{i}^{-r_{i}} / \mathfrak{t}_{i}^{1}\right) \subset \Omega_{\widehat{\mathcal{M}}}^{1}\left(\mathfrak{P}_{i}^{-r_{i}} / \mathfrak{P}_{i}^{1}\right)$.

Identify $\widetilde{\mathcal{M}}_{i}$ with $\mathfrak{V}_{j} \times_{U_{j}} \mathrm{GL}_{n}(\mathbb{C})$ as in Lemma 3.8. Throughout this section, we will use $m_{j}=\left(v_{j}, g_{j}\right) \in \mathfrak{V}_{j} \times_{U_{j}} \mathrm{GL}_{n}(\mathbb{C})$ to denote a point in $\widetilde{\mathcal{M}}_{i}$ and $m=\left(m_{j}\right)_{j \in I}$ to denote a point in $\widehat{\mathcal{M}} \subset \prod_{i \in I} \widetilde{\mathcal{M}}_{i}$. Let $\psi_{i}: \widehat{\mathcal{M}} \rightarrow U_{i} \backslash \mathrm{GL}_{n}(\mathbb{C})$ be the composition of the projections $\widehat{\mathcal{M}} \rightarrow \widetilde{\mathcal{M}}_{i}$ and $\tilde{\psi}_{i}: \widetilde{\mathcal{M}}_{i} \rightarrow U_{i} \backslash \mathrm{GL}_{n}(\mathbb{C})$.

We are now ready to define a collection of differential forms on $\widehat{\mathcal{M}}$.

(1) Define an endomorphism of $\mathfrak{t}_{i}$ by

$$
\delta_{\mathrm{e}}{ }^{\prime}\left(t_{-j} \varpi_{i}^{-j}\right)= \begin{cases}\frac{-e}{j} t_{-j} \varpi_{i}^{-j} & \text { if } j \neq 0 ; \\ 0 & \text { if } j=0 .\end{cases}
$$

Note that if $t=\sum_{i=-N}^{\infty} t_{i} \varpi_{i}$, then $\delta_{e}\left(\delta_{\mathrm{e}}{ }^{\prime}(t)\right)=t-t_{0}$. We obtain an induced $\operatorname{map} \delta_{\mathrm{e}}{ }^{\prime}: \Omega_{\widehat{\mathcal{M}}}^{*}\left(\mathfrak{t}_{i}^{-r_{i}} / \mathfrak{t}_{i}^{1}\right) \rightarrow \Omega_{\widehat{\mathcal{M}}}^{*}\left(\mathfrak{t}_{i}^{-r_{i}} / \mathfrak{t}_{i}^{1}\right)$.

We shall clarify the dictionary between this notation and the notation in Section 4.1 Set $A_{\widetilde{\mathcal{M}}, i}=\delta_{\mathrm{e}}{ }^{\prime}\left(d \hat{\Gamma}_{i}\right) \in \Omega_{\widehat{\mathcal{M}}}^{1}\left(\mathfrak{t}_{i}^{-r_{i}} / \mathfrak{t}_{i}^{1}\right)$. If $\nu=\frac{d z_{i}}{z_{i}}$, the first part of Lemma 4.1 implies that

$$
\tau_{\nu} A_{\widetilde{\mathcal{M}}, i}-\left[A_{\widetilde{\mathcal{M}}, i}, \hat{\Gamma}_{i \nu}\right]=d \hat{\Gamma}_{i \nu}-d \hat{\Gamma}_{i}^{0}+\mathfrak{t}_{i}^{1} .
$$

Below, we will apply Proposition 4.2 several times with $A_{\widetilde{\mathcal{M}}, i}$ and $\hat{\Gamma}_{i \nu}-\hat{\Gamma}_{i}^{0}$ playing the roles of $A_{\Delta}$ and $\tilde{A}_{\nu}$ respectively.

(2) Recall, from (3.1), that $\left(\mathfrak{P}_{i} / \mathfrak{P}_{i}^{r_{i}+1}\right)_{\mathrm{reg}}^{\vee} \cong \mathcal{A}_{i} \times_{T_{i}^{1}} P_{i}^{1} / P_{i}^{r+1}$. Given $m_{i} \in$ $\widetilde{\mathcal{M}}_{i}$, we may write $\pi_{\mathfrak{P}_{i}}\left(v_{i}\right)=\left(a_{i}, p_{i}\right) \in \mathcal{A}_{i} \times_{T_{i}^{1}} P_{i}^{1} / P_{i}^{r_{i}+1}$. Thus, $a_{i}$ is the image of $v_{i}$ under the map $\Gamma_{i}$ from Proposition 3.9, and $p_{i}$ is characterized by $\operatorname{Ad}^{*}\left(p_{i}\right)\left(\pi_{\mathfrak{P}_{i}}\left(v_{i}\right)\right)=a_{i}$. Define a bundle $B_{i}=\mathfrak{P}_{i}^{-r} / \mathfrak{P}_{i}^{1} \times_{U_{i}} \mathrm{GL}_{n}(\mathbb{C})$ 
over $U_{i} \backslash \mathrm{GL}_{n}(\mathbb{C})$, where $U_{i}$ acts on $\mathrm{GL}_{n}(\mathbb{C})$ by left multiplication and on $\mathfrak{P}_{i}^{-r} / \mathfrak{P}_{i}^{1}$ by Ad. We define $\Phi_{i} \in \Omega_{\widehat{\mathcal{M}}}^{1}\left(\psi_{i}^{*}\left(B_{i}\right)\right)$ by

$$
\Phi_{i}(m)=\operatorname{Ad}\left(p_{i}^{-1}\right)\left(A_{\widetilde{\mathcal{M}}, i}\right) .
$$

Note that $\Phi_{i}$ is a well defined section of $\Omega_{\widehat{\mathcal{M}}}^{1}\left(\psi_{i}^{*}\left(B_{i}\right)\right)$ : if $u \in U_{i}$, then $u\left(a_{i}, p_{i}\right)=\left(a_{i}, p_{i} u^{-1}\right)$; it follows that $\operatorname{Ad}(u) \Phi_{i}(u m)=\Phi_{i}(m)$.

(3) We define a third form $\Upsilon^{0}$ on $\widehat{\mathcal{M}}$ which has coefficients in $H^{0}\left(\mathbb{P}^{1} ; \mathfrak{g l}_{n \mathbb{P}^{1}}^{\mathrm{r}_{\mathrm{e}}}\right)$. The form

$$
\bar{\Phi}_{i}=\operatorname{Ad}\left(g_{i}^{-1}\right) \Phi_{i}+\Omega_{\widehat{\mathcal{M}}}^{1}\left(\mathfrak{g}_{i} / \mathfrak{P}_{i}^{1}\right) \in \Omega_{\widehat{\mathcal{M}}}^{1}\left(\left(\mathfrak{P}_{i}^{-r_{i}}\right)^{g_{i}} / \mathfrak{g}_{i}\right)
$$

is well defined on $\widehat{\mathcal{M}}$, so the product $\bar{\Phi}=\left(\bar{\Phi}_{i}\right)_{i \in I}$ determines a form in $\Omega_{\widehat{\mathcal{M}}}^{1}\left(\prod_{i \in I}\left(\left(\mathfrak{P}_{i}^{-r_{i}}\right)^{g_{i}} / \mathfrak{g}_{i}\right)\right)$. Using Definition 4.8, we set $\Upsilon^{0}=\varsigma(\bar{\Phi}) \in$ $\Omega_{\widehat{\mathcal{M}}}^{1}\left(H^{0}\left(\mathbb{P}^{1} ; \mathfrak{g}_{n \mathbb{P}^{1}}^{\mathbf{r}_{\mathbf{e}}}\right)\right)$.

(4) The next form, $\Theta_{i}$, is defined on $\widehat{\mathcal{M}}$ with coefficients in $\psi_{i}^{*}\left(T\left(U_{i} \backslash \mathrm{GL}_{n}(\mathbb{C})\right)\right)$. Here, we identify $T\left(U_{i} \backslash \mathrm{GL}_{n}(\mathbb{C})\right)$ with $\mathfrak{u}_{i} \backslash \mathfrak{g l}_{n}(\mathbb{C}) \times_{U_{i}} \mathrm{GL}_{n}(\mathbb{C})$ in the usual way. Define a section of $\Omega_{\widehat{\mathcal{M}}}^{1}\left(\psi_{i}^{*} T\left(U_{i} \backslash \mathrm{GL}_{n}(\mathbb{C})\right)\right)$ by

$$
\Theta_{i}(m)=\left(d g_{i}\right) g_{i}^{-1}+\bar{\phi}_{i}\left(\Phi_{i}\right)-\bar{\phi}_{i}\left(\operatorname{Ad}\left(g_{i}\right)\left(\Upsilon^{0}\right)\right)+\Omega_{\widehat{\mathcal{M}}}^{1}\left(\mathfrak{u}_{i}\right)
$$

where $\bar{\phi}$ is the function defined in (4.5). The form $\Theta_{i}$ is a well defined. By construction, $\bar{\phi}_{i}\left(\Phi_{i}\right)$ is a well defined section of $\Omega_{\widehat{\mathcal{M}}}^{1}\left(\psi_{i}^{*} T\left(U_{i} \backslash \mathrm{GL}_{n}(\mathbb{C})\right)\right)$. Furthermore, $\operatorname{Ad}\left(u_{i}\right) \bar{\phi}_{i}\left(\operatorname{Ad}\left(g_{i}\right) \Upsilon^{0}\right)\left(u_{i} m\right)=\bar{\phi}_{i}\left(\operatorname{Ad}\left(g_{i}\right) \Upsilon^{0}\right)(m)$ and

$$
d\left(u_{i} g_{i}\right) g_{i}^{-1} u_{i}^{-1} \in \operatorname{Ad}\left(u_{i}\right)\left(\left(d g_{i}\right) g_{i}^{-1}\right)+\Omega_{\widehat{\mathcal{M}}}^{1}\left(\mathfrak{u}_{i}\right) .
$$

(5) By Theorem 3.16, each point of $\widehat{\mathcal{M}}$ determines a unique form $\alpha \nu \in H^{0}\left(\mathbb{P}^{1} \backslash \mathbf{x} ; \Omega_{\mathbb{P}^{1}}^{1}\left(\mathfrak{g l}_{n \mathbb{P}^{1}}\right)\right)$. In particular, $d \alpha$ is a one-form with coefficients in $H^{0}\left(\mathbb{P}^{1} \backslash \mathbf{x} ; \mathfrak{g l}_{n \mathbb{P}^{1}}\right)$. Note that $d \alpha$ depends on the choice of global form $\nu$.

(6) We define the last collection of forms on $\widehat{\mathcal{M}}$ to have coefficients in $\left(\mathfrak{g}_{i}^{-r_{i}^{\prime}} \frac{d z_{i}}{z_{i}}\right) /\left(\mathfrak{g}_{i}^{1} \frac{d z_{i}}{z_{i} \nu}\right)$, where $\mathbf{r}_{\mathbf{e}}=\left(r_{i}^{\prime}\right)_{i \in I}$ as in Definition 4.7. Let $\Xi_{i}$ be defined on $\widehat{\mathcal{M}}$ by

$$
\Xi_{i}\left(\left(v_{j}, g_{j}\right)_{j \in I}\right)=\tau_{\nu} \Upsilon^{0}-d \alpha-\left[\Upsilon^{0}, \alpha\right]+\Omega_{\widehat{\mathcal{M}}}^{1}\left(\mathfrak{g}_{i}^{1} \frac{d z_{i}}{z_{i} \nu}\right) .
$$

Intuitively, we have a framed global deformation on any neighborhood of $\widehat{\mathcal{M}}$ isomorphic to an analytic polydisk determined by $\mathbf{g}=\left(g_{i}\right)_{i \in I}$ and $\tilde{\nabla}=d+\alpha \nu$ (see Definition 4.6). Here, $\Upsilon^{0}$ represents the $d y$ term of the connection (up to a section of $\Omega_{\widehat{M}}^{1}\left(\mathfrak{g l}_{n}(\mathbb{C})\right)$ ). The form $\Xi_{i}$ corresponds to the expansion, in non-positive degrees of $z_{i}$, of the $d z \wedge d y$ term of the curvature. Moreover, the vanishing of $\Theta_{i}$ gives a relation on the framing $g_{i}$ analogous to part (11) of Theorem 4.12 .

5.2. The Differential Ideal. We are now ready to construct a differential ideal $\mathcal{I}$ on $\widehat{\mathcal{M}}$ corresponding to the isomonodromy equations in Section 4 Throughout, we fix sections $\hat{g}_{i}$ of the trivial $\mathrm{GL}_{n}\left(\mathfrak{o}_{i}\right)$-bundle over $\widehat{\mathcal{M}}$ such that $\hat{g}_{i} g_{i}^{-1} \in P_{i}^{1}$ and $\hat{g}_{i} \cdot \alpha=$ $\hat{\Gamma}_{i \nu}$. We write $\hat{p}_{i}=\hat{g}_{i} g_{i}^{-1}$. Observe that $\operatorname{Ad}\left(g_{i}\right)\left(\hat{g}_{i}^{-1} d \hat{g}_{i}\right) \in\left(d g_{i}\right) g_{i}^{-1}+\Omega_{\widehat{\mathcal{M}}}^{1}\left(\mathfrak{P}_{i}^{1}\right)$ and $p_{i}=\hat{p}_{i} P_{i}^{r_{i}+1}$.

For convenience, we will take $A_{\widetilde{\mathcal{M}}, i}^{\prime} \in \Omega_{\widehat{\mathcal{M}}}^{1}\left(\mathfrak{t}_{i}^{-r}\right)$ to be the lift of $A_{\widetilde{\mathcal{M}}, i}$ which has zero components in positive degrees. By Lemma 4.1, this lift has the property

$$
\tau_{\nu} A_{\widetilde{\mathcal{M}}, i}^{\prime}+\left[\hat{\Gamma}_{i \nu}, A_{\widetilde{\mathcal{M}}, i}^{\prime}\right]=d \hat{\Gamma}_{i \nu}-d \hat{\Gamma}_{i}^{0} .
$$


Moreover, $d A_{\widetilde{\mathcal{M}}, i}^{\prime}=0$, since $A_{\widetilde{\mathcal{M}}, i}^{\prime}=\delta_{\mathrm{e}}{ }^{\prime}\left(d \hat{\Gamma}_{i \nu}\right)$. The same holds for $A_{\widetilde{\mathcal{M}}, i}$.

If $B$ is a vector bundle over $\widehat{\mathcal{M}}, \sigma \in \Omega_{\widehat{\mathcal{M}}}^{*}(B)$ and $\mathcal{J}$ is a differential ideal on $\widehat{\mathcal{M}}$, we will use the shorthand " $\sigma \in \mathcal{J}$ " to mean $\sigma \in \mathcal{I} \otimes_{\mathcal{O}_{\widehat{\mathcal{M}}}} B$. If $B^{\prime}$ is another bundle and $f: B \rightarrow B^{\prime}$ is a bundle map, then $\sigma \in \mathcal{J}$ implies that $f(\sigma) \in \mathcal{J}$. In particular, this is the case if the bundles are trivial with $f$ induced by a $\mathbb{C}$-linear map on their fibers. We also say that $\mathcal{J}$ is generated by $\sigma$ if it is locally generated by the coefficients of $\sigma$ in a trivialization of $B$.

Throughout, we may assume that $\nu=\frac{d z_{i}}{z_{i}}$ when working with $\Xi_{i}$ and $\Theta_{i}$. Let $\chi_{i}=\phi_{i}\left(\operatorname{Ad}\left(\hat{g}_{i}^{-1}\right) A_{\widetilde{\mathcal{M}}, i}^{\prime}+\hat{g}_{i}^{-1} d \hat{g}_{i}-\Upsilon^{0}\right) \in \Omega_{\widehat{\mathcal{M}}}^{1}\left(\mathfrak{g l}_{n}(\mathbb{C})\right)$. Note that $\operatorname{Ad}\left(g_{i}\right)\left(\chi_{i}\right)+\Omega_{\widehat{\mathcal{M}}}^{1}\left(\mathfrak{u}_{i}\right)$ is a well-defined section of the bundle $B_{i}$ from the previous section. Choose some $j \in$ I. Let $\mathcal{I}$ be the differential ideal on $\widehat{\mathcal{M}}$ is generated by $\Xi_{i}-\left[\chi_{j}, \alpha\right], \Theta_{i}-\operatorname{Ad}\left(g_{i}\right)\left(\chi_{j}\right)$, and $d \hat{\Gamma}_{i}^{0}$ for all $i \in I$. We will show late that this definition is independent of the choice of $j$.

\section{Theorem 5.1.}

(1) The differential ideal $\mathcal{I}$ is a Pfaffian ideal which satisfies the Frobenius integrability condition $d \mathcal{I} \subset \mathcal{I}$ and therefore determines a foliation of $\widehat{\mathcal{M}}(\mathbf{x}, \mathbf{P}, \mathbf{r})$. Moreover, any map $f: \Delta \rightarrow \widehat{\mathcal{M}}$ corresponds to a framed integrable deformation if and only if $f^{*} \mathcal{I}=0$.

(2) The ideal $\mathcal{I}$ is the pullback of an integrable Pfaffian differential ideal $\overline{\mathcal{I}}$ on $\widetilde{\mathcal{M}}(\mathbf{P}, \mathbf{x}, \mathbf{r})$, and any map $f: \Delta \rightarrow \widetilde{\mathcal{M}}$ corresponds to a framed integrable deformation if and only if $f^{*} \overline{\mathcal{I}}=0$.

We will prove this theorem in the subsequent two sections. The remainder of this section is devoted to a local analysis of the ideal $\mathcal{I}$ which will be useful later on.

Over a sufficiently small neighborhood $N_{i} \subset U_{i} \backslash \mathrm{GL}_{n}(\mathbb{C})$, we may choose a smooth algebraic slice of coset representatives $\sigma_{i}: N_{i} \rightarrow \mathrm{GL}_{n}(\mathbb{C})$. Let $\widetilde{N}_{i} \subset \mathrm{GL}_{n}(\mathbb{C})$ be the inverse image of $N_{i}$, and let $N=\bigcap_{i \in I} \psi_{i}^{-1}\left(N_{i}\right) \subset \widehat{\mathcal{M}}$.

For brevity, we will write $g_{i}^{\sigma}=\sigma_{i}\left(U_{i} g_{i}\right)$. When $\chi \in \mathfrak{g l}_{n}(\mathbb{C})=T_{g_{i}^{\sigma}} \mathrm{GL}_{n}(\mathbb{C})$, we write $\chi^{\sigma} \in \mathfrak{g l}_{n}(\mathbb{C})$ for the image of $\chi$ under the tangent map corresponding to the composition $\widetilde{N}_{i} \rightarrow N_{i} \stackrel{\sigma_{i}}{\longrightarrow} \widetilde{N}_{i}$. We also define $\Theta_{i}^{\sigma} \in \Omega_{N}^{1}\left(\mathfrak{g l}_{n}(\mathbb{C})\right)$ by

$$
\Theta_{i}^{\sigma}=\left(g_{i}^{\sigma}\right)^{-1} d g_{i}^{\sigma}+T \sigma_{i}\left(\bar{\phi}_{i}\left(\Phi_{i}\right)-\bar{\phi}_{i}\left(\operatorname{Ad}\left(g_{i}\right)\left(\Upsilon^{0}\right)\right)\right) \text {. }
$$

Observe that $\operatorname{Ad}\left(g_{i}^{\sigma}\right)\left(\Theta_{i}^{\sigma}\right)+\Omega_{N}^{1}\left(\mathfrak{u}_{i}\right)=\left.\Theta_{i}\right|_{N}$; indeed, $\Theta_{i}^{\sigma}=T \sigma_{i}\left(\Theta_{i}\right)$.

We define a map $\xi_{i}: \mathfrak{g}_{i} \rightarrow \Omega_{N}^{1}$ using the residue-trace pairing in (2.1):

$$
\xi_{i}(X)=\left\langle\operatorname{Ad}\left(g_{i}^{\sigma}\right)\left(\Xi_{i}-\left[\Theta_{i}^{\sigma}, \alpha\right]\right), X\right\rangle_{\nu} .
$$

Lemma 5.2. Let $\mathcal{J}$ be a differential ideal on $N \subset \widehat{\mathcal{M}}$, and let $\Xi$ be a section of $\Omega_{N}^{1}\left(\mathfrak{P}^{-R} / \mathfrak{g}^{1}\right)$ for some integer $R>0$. Define $\xi \in \Omega_{N}^{1}\left(\mathfrak{g}^{\vee}\right)$ by $\xi(X)=\langle\Xi, X\rangle_{\nu}$. Then $\xi(\mathfrak{g}) \subset \mathcal{J}$ if and only if $\Xi \in \mathcal{J}$. Furthermore, $\xi\left(\mathfrak{P}^{s}\right) \in \mathcal{J}$ if and only if $\Xi \in \mathcal{J}+\Omega_{N}^{1}\left(\mathfrak{P}^{-s+1}\right)$

Proof. By assumption, $\mathfrak{P}^{R+1} \subset \operatorname{ker}(\xi)$, so $\xi$ induces an element $\bar{\xi} \in \operatorname{Hom}_{\mathbb{C}}\left(\mathfrak{g} / \mathfrak{P}^{R+1}, \Omega_{N}^{1}\right) \cong$ $\Omega_{N}^{1}\left(\left(\mathfrak{g} / \mathfrak{P}^{R+1}\right)^{\vee}\right)$. Note that $\xi(\mathfrak{g}) \subset \mathcal{J}$ if and only $\bar{\xi} \in \mathcal{J}$. Moreover, $\bar{\xi}$ and $\Xi$ correspond under the isomorphism $\left(\mathfrak{g} / \mathfrak{P}^{R+1}\right)^{\vee} \cong \mathfrak{P}^{-R} / \mathfrak{g}^{1}$ given by the perfect pairing $\langle,\rangle_{\nu}$, so $\bar{\xi} \in \mathcal{J}$ if and only if $\Xi \in \mathcal{J}$. The first result now follows. A similar argument, replacing $\mathfrak{g}$ with $\mathfrak{P}^{s}$, proves the second statement. 
We will write $\hat{\rho}$ for the action of $\mathrm{GL}_{n}(\mathbb{C})$ on $\prod_{i \in I} \widetilde{\mathcal{M}}_{i}$. (This is the product over the action of $\mathrm{GL}_{n}(\mathbb{C})$ on each factor $\widetilde{\mathcal{M}}(P, r)$ from Lemma 3.13]) Define $\mathrm{T}_{\mathfrak{g r}_{n}} \subset$ $\prod_{i \in I} \mathrm{~T}\left(\widetilde{\mathcal{M}}_{i}\right)$ to be the subbundle of $\mathrm{T}\left(\prod_{i \in I} \widetilde{\mathcal{M}}_{i}\right)$ consisting of vectors tangent to the $\mathrm{GL}_{n}(\mathbb{C})$-orbits. Since the action of $\mathrm{GL}_{n}(\mathbb{C})$ is free, this is an integrable subbundle. Moreover, the action map of $\hat{\rho}$ gives an isomorphism between the trivial $\mathfrak{g l}_{n}(\mathbb{C})$ bundle on $\prod_{i \in I} \widetilde{\mathcal{M}}_{i}$ and $\mathrm{T}_{\mathfrak{g l}_{n}}$.

We may identify the preimage $\tilde{\psi}_{i}^{-1}\left(N_{i}\right)$ of $N_{i}$ in $\widetilde{\mathcal{M}}_{i}$ with $\mathfrak{V}_{i} \times N_{i}$ via

$$
\left(v, g_{i}\right) \mapsto\left(\operatorname{Ad}\left(g_{i}^{\sigma} g_{i}^{-1}\right) v, U_{i} g_{i}\right) .
$$

Thus, there is a local isomorphism

$$
\mathrm{T}\left(\tilde{\psi}_{i}^{-1}\left(N_{i}\right)\right) \cong \mathrm{T}\left(\mathfrak{V}_{i}\right) \times \mathrm{T}\left(N_{i}\right) .
$$

This equation induces a local bundle map $\mathrm{p}_{\mathrm{i}}^{\mathrm{U}}:\left.\left.\mathrm{T}_{\mathfrak{g l}_{n}}\right|_{N} \rightarrow \mathrm{T}\left(\mathfrak{V}_{i}\right) \subset T\left(\prod_{i \in I} \tilde{\psi}^{-1}\left(N_{i}\right)\right)\right|_{N}$. We also have a global bundle map $\mathrm{p}_{\mathrm{i}}^{\mathrm{L}}:\left.\mathrm{T}_{\mathfrak{g l}_{n}}\right|_{N} \rightarrow \psi_{i}^{-1} T\left(U_{i} \backslash \mathrm{GL}_{n}(\mathbb{C})\right)$.

Recall from Lemma 3.8 that $\mathfrak{V}_{i}$ is open dense in $Z_{i}=\left(\mathfrak{g}_{i} / \widetilde{W}_{r_{i}}\right)^{\vee}$. Therefore, $T\left(\mathfrak{V}_{i}\right) \cong \mathfrak{V}_{i} \times Z_{i}$.

Lemma 5.3. Let $\chi$ be a section of $\Omega_{N}^{1}\left(\mathrm{~T}_{\mathfrak{g l}_{n}}\right)$. Then, $\mathrm{p}_{\mathrm{i}}^{\mathrm{L}}(\chi)=-\operatorname{Ad}\left(g_{i}\right)(\chi)+\mathfrak{u}_{i} \in$ $\Omega_{N}^{1}\left(B_{i}\right)$. Moreover, $\mathrm{p}_{\mathrm{i}}^{\mathrm{U}}(\chi) \in \Omega_{N}^{1}\left(Z_{i}^{\vee}\right)$ is the functional defined by $\operatorname{Ad}\left(g_{i}^{\sigma}\right)\left(\left[\chi-\chi^{\sigma}, \alpha\right]\right)$.

Proof. The action of $\mathrm{GL}_{n}(\mathbb{C})$ on $U_{i} \backslash \mathrm{GL}_{n}(\mathbb{C})$ is the usual (left) action, $h\left(U_{i} g\right)=$ $U_{i} g h^{-1}$. The first statement follows. The map $\widetilde{N}_{i} \rightarrow U_{i}$ defined by $g \mapsto g\left(g^{\sigma}\right)^{-1}$ has tangent map $X \mapsto \operatorname{Ad}\left(g^{\sigma}\right)\left(X-X^{\sigma}\right)$. Since the projection from the $\tilde{\psi}_{i}\left(N_{i}\right) \subset \widetilde{\mathcal{M}}_{i}$ onto $\mathfrak{V}_{i}$ has the form $\left(v_{i}, g_{i}\right) \mapsto \operatorname{Ad}\left(g_{i}^{\sigma} g_{i}^{-1}\right)\left(v_{i}\right)$, it follows that $\mathrm{p}_{\mathrm{i}}^{\mathrm{U}}(\chi)=\left[\operatorname{Ad}\left(g_{i}^{\sigma}\right)(\chi-\right.$ $\left.\left.\left.\chi^{\sigma}\right), \operatorname{Ad}\left(g_{i}^{\sigma}\right)(\alpha)\right]\right)$.

Choose a vector space lift $\widehat{Z}_{i} \subset \mathfrak{g}_{i}$ of $\mathfrak{g}_{i} / \widetilde{W}_{r_{i}}$. Define $\Xi_{i}^{\mathfrak{V}} \in\left(\widehat{Z}_{i}\right)^{\vee} \cong Z_{i}$ by $\Xi_{i}^{\mathfrak{V}}=\left.\xi_{i}\right|_{\widehat{Z}_{i}}$, using the notation in (5.5). In other words, $\Xi_{i}^{\mathfrak{N}}$ is the functional on $\widehat{Z}_{i}$ determined by $\operatorname{Ad}\left(g_{i}^{\sigma}\right)\left(\Xi_{i}-\left[\Theta_{i}^{\sigma}, \alpha\right]\right)$. The $I$-tuple $\tilde{\kappa}=\left(-\Xi_{i}^{\mathfrak{N}}, \Theta_{i}\right)_{i \in I}$ determines a section of $\Omega_{N}^{1}\left(\prod_{i \in I} \mathrm{~T}\left(\widetilde{\mathcal{M}}_{i}\right)\right)$. We define $\kappa$ to be the differential form on $\Omega_{N}^{1}\left(\left(\prod_{i \in I} \mathrm{~T}\left(\widetilde{\mathcal{M}}_{i}\right)\right) / \mathrm{T}_{\mathfrak{g l}_{n}}\right)$ determined by the image of $\tilde{\kappa}$.

Definition 5.4. We define $\mathcal{I}^{\sigma}$ to be the differential ideal on $N$ determined by the coefficients of $\kappa$ and by $d \hat{\Gamma}_{i}^{0}$ for each $i \in I$.

We wish to show that $\mathcal{I}^{\sigma}$ is the restriction of $\mathcal{I}$ to $N$.

Lemma 5.5. Suppose that $\mathcal{J}$ is a differential ideal on $N$ containing $d \hat{\Gamma}_{i}^{0}$ and $\chi$ is a section of $\Omega_{N}^{1}\left(\mathrm{~T}_{\mathfrak{g l}}\right)$. If

$$
\operatorname{Ad}\left(g_{i}\right)\left(\Upsilon^{0}\right)+\operatorname{Ad}\left(g_{i}\right)(\chi)-\left(d g_{i}\right) g_{i}^{-1} \in \Phi_{i}+\mathcal{J}+\Omega_{N}^{1}\left(\mathfrak{P}_{i}^{1}\right),
$$

then $\operatorname{Ad}\left(g_{i}\right)\left(\Xi_{i}-[\chi, \alpha]\right) \in \Omega_{N}^{1}\left(\left[\mathfrak{P}_{i}^{1}, \operatorname{Ad}\left(g_{i}\right)(\alpha)\right]+\mathfrak{P}_{i}^{1} \frac{d z_{i}}{z_{i}}\right)+\mathcal{J}$.

Proof. Without loss of generality, let $\nu=\frac{d z_{i}}{z_{i}}$. By construction, $\tau_{\nu}\left(A_{\widetilde{\mathcal{M}}, i}^{\prime}\right)-d \hat{\Gamma}_{i \nu}+$ $d \hat{\Gamma}_{i}^{0}+\left[\hat{\Gamma}_{i \nu}, A_{\widetilde{\mathcal{M}}, i}^{\prime}\right]=0$. Write $\alpha^{\prime}=\hat{g}_{i}^{-1} \cdot\left(\hat{\Gamma}_{i \nu}-\hat{\Gamma}_{i}^{0}\right)$, so $\alpha-\alpha^{\prime}=\operatorname{Ad}\left(\hat{g}_{i}^{-1}\right)\left(\hat{\Gamma}_{i}^{0}\right)$. Set $\Upsilon=\operatorname{Ad}\left(\hat{g}_{i}^{-1}\right) A_{\widetilde{\mathcal{M}}, i}^{\prime}+\hat{g}_{i}^{-1} d \hat{g}_{i}$.

By Proposition 4.2, $\hat{g}_{i}^{-1} \cdot\left(\left(\hat{\Gamma}_{i \nu}-\hat{\Gamma}_{i}^{0}\right) \nu+A_{\widetilde{\mathcal{M}}, i}^{\prime}\right)+\hat{g}_{i}^{-1} d \hat{g}_{i}=\alpha^{\prime} \nu+\Upsilon$ determines a flat connection on $\operatorname{Spec}\left(F_{i}\right) \times N$. Therefore, $\tau_{\nu}(\Upsilon)-d \alpha^{\prime}+\left[\alpha^{\prime}, \Upsilon\right]=0$. It follows 
that

$$
\tau_{\nu}(\Upsilon)-d \alpha+[\alpha, \Upsilon]=-\operatorname{Ad}\left(\hat{g}_{i}^{-1}\right) d \hat{\Gamma}_{i}^{0} \in \mathcal{J}
$$

By definition, $\operatorname{Ad}\left(\hat{g}_{i}^{-1}\right) A_{\widetilde{\mathcal{M}}, i}+\Omega_{N}^{1}\left(\left(\mathfrak{P}_{i}^{1}\right)^{g_{i}}\right)=\operatorname{Ad}\left(g_{i}^{-1}\right) \Phi_{i}$ and $\hat{g}_{i}^{-1} d \hat{g}_{i} \in g_{i}^{-1} d g_{i}+$ $\Omega_{N}^{1}\left(\left(\mathfrak{P}_{i}^{1}\right)^{g_{i}}\right)$. Therefore, subtracting the above expression from $\Xi_{i}-[\chi, \alpha]$ and using the hypothesis yields

$$
\begin{aligned}
\Xi_{i}-[\chi, \alpha] \in \tau_{\nu}\left(\Upsilon^{0}+\chi-\operatorname{Ad}\left(\hat{g}_{i}^{-1}\right) A_{\widetilde{\mathcal{M}}, i}-\hat{g}_{i}^{-1} d \hat{g}_{i}\right)- \\
{\left[\Upsilon^{0}+\chi-\operatorname{Ad}\left(\hat{g}_{i}^{-1}\right) A_{\widetilde{\mathcal{M}}, i}-\hat{g}_{i}^{-1} d \hat{g}_{i}, \alpha\right]+\Omega_{N}^{1}\left(\left(\mathfrak{P}_{i}^{1}\right)^{g_{i}}\right)+\mathcal{J} . }
\end{aligned}
$$

The lemma follows after applying $\operatorname{Ad}\left(g_{i}\right)$ to both sides of the equation above.

Lemma 5.6. Let $\mathcal{J}$ be a differential ideal on $N$ satisfying the following property: there exists a section $\chi$ of $\Omega_{N}^{1}\left(\mathrm{~T}_{\mathfrak{g l}_{n}}\right)$ such that $\Theta_{i}+\mathrm{p}_{\mathrm{i}}^{\mathrm{L}}(\chi) \in \mathcal{J}$ for all $i$. Then,

$$
\operatorname{Ad}\left(g_{i}\right)\left(\Upsilon^{0}+\chi\right) \in \operatorname{Ad}\left(\hat{p}_{i}^{-1}\right) A_{\widetilde{\mathcal{M}}, i}^{\prime}+\left(d g_{i}\right) g_{i}^{-1}+\Omega_{\widetilde{\mathcal{M}}}^{1}\left(\mathfrak{P}_{i}^{1}\right)+\mathcal{J}
$$

Proof. We note that $\Upsilon^{0}-\operatorname{Ad}\left(\hat{g}_{i}^{-1}\right)\left(A_{\widetilde{\mathcal{M}}, i}^{\prime}\right) \in \Omega_{\widehat{\mathcal{M}}}^{1}\left(\mathfrak{g}_{i}\right)$ by construction. Since the map $\bar{\phi}_{i}$ induces an isomorphism $\mathfrak{P}_{i}^{1} \backslash \mathfrak{g}_{i} \cong \mathfrak{u}_{i} \backslash \mathfrak{g l}_{n}(\mathbb{C})$, it suffices to show

$$
\bar{\phi}_{i}\left(\operatorname{Ad}\left(g_{i}\right)\left(\Upsilon^{0}+\chi\right)-\operatorname{Ad}\left(\hat{p}_{i}^{-1}\right)\left(A_{\widetilde{\mathcal{M}}, i}^{\prime}\right)-\left(d g_{i}\right) g_{i}^{-1}\right) \in \mathcal{J} \text {. }
$$

This is equal to $-\Theta_{i}+\operatorname{Ad}\left(g_{i}\right)(\chi)+\Omega_{N}^{1}\left(\mathfrak{u}_{i}\right)$ as a section of $\Omega_{N}^{1}\left(\psi_{i}^{*} T\left(U_{i} \backslash \mathrm{GL}_{n}(\mathbb{C})\right)\right)$. By Lemma 5.3 .

$$
-\Theta_{i}+\operatorname{Ad}\left(g_{i}\right)(\chi)+\Omega_{N}^{1}\left(\mathfrak{u}_{i}\right)=-\Theta_{i}-\mathrm{p}_{\mathrm{i}}^{\mathrm{L}}(\chi) \in \mathcal{J} .
$$

It follows that $\operatorname{Ad}\left(g_{i}\right)\left(\Upsilon^{0}+\chi\right) \in \operatorname{Ad}\left(\hat{p}_{i}^{-1}\right)\left(A_{\widetilde{\mathcal{M}}, i}^{\prime}\right)+\left(d g_{i}\right) g_{i}^{-1}+\Omega_{\widehat{\mathcal{M}}}^{1}\left(\mathfrak{P}_{i}^{1}\right)+\mathcal{J}$.

Proposition 5.7. On a sufficiently small neighborhood $N$, there exists a section $\chi$ of $\Omega_{N}^{1}\left(\mathrm{~T}_{\mathfrak{g l}_{n}}\right)$ such that $\tilde{\kappa}+\chi \in \mathcal{I}^{\sigma}$. Any such $\chi$ will satisfy the conditions of Lemma 5.6] for $\mathcal{J}=\mathcal{I}^{\sigma}$; furthermore, $\mathcal{I}^{\sigma}$ is generated by $\Xi_{i}-[\chi, \alpha], \Theta_{i}+\mathrm{p}_{\mathrm{i}}^{\mathrm{L}}(\chi)$, and $d \hat{\Gamma}_{i}^{0}$. In particular, $\mathcal{I}^{\sigma}$ is independent of the choice of lift $\widehat{Z}_{i}$.

For the next few results, we will assume that $N$ is taken to be sufficiently small as in the statement of the lemma. However, we shall see below in Corollary 5.10 that we may choose $\chi$ to be the restriction of the globally defined $\chi_{j}$.

Proof. Shrinking $N$ if necessary, we may choose a direct sum decomposition $\left.\prod_{i \in I} \mathrm{~T}\left(\widetilde{\mathcal{M}}_{i}\right)\right)\left.\right|_{N} \cong$ $\left.\mathrm{T}_{\mathfrak{g l}_{n}}\right|_{N} \oplus \mathrm{T}_{\mathfrak{g l}_{n}}^{\perp}$. Here, $\left.\mathrm{T}_{\mathfrak{g l}_{n}}^{\perp} \cong\left(\left(\prod_{i \in I} \mathrm{~T}\left(\widetilde{\mathcal{M}}_{i}\right)\right) / \mathrm{T}_{\mathfrak{g l}_{n}}\right)\right|_{N}$. Thus, we may choose a section $\chi$ of $\Omega_{N}^{1}\left(\mathrm{~T}_{\mathfrak{g l}_{n}}\right)$ such that $\tilde{\kappa}+\chi \in \mathcal{I}^{\sigma}$. In particular, $\Theta_{i}+\mathrm{p}_{\mathrm{i}}^{\mathrm{L}}(\chi) \in \mathcal{I}^{\sigma}$ and $-\Xi_{i}^{\mathfrak{V}}+\mathrm{p}_{\mathrm{i}}^{\mathrm{U}}(\chi) \in \mathcal{I}^{\sigma}$. By Lemma 5.6.

$$
\operatorname{Ad}\left(g_{i}\right)\left(\Upsilon^{0}\right)+\operatorname{Ad}\left(g_{i}\right)(\chi)-\left(d g_{i}\right) g_{i}^{-1} \in \operatorname{Ad}\left(\hat{p}_{i}^{-1}\right)\left(A_{\widetilde{\mathcal{M}}, i}^{\prime}\right)+\mathcal{I}^{\sigma}+\Omega_{\widetilde{\mathcal{M}}}^{1}\left(\mathfrak{P}_{i}^{1}\right)
$$

The right hand side is equal to $\Phi_{i}+\mathcal{I}^{\sigma}+\Omega_{\widehat{\mathcal{M}}}^{1}\left(\mathfrak{P}_{i}^{1}\right)$. We may conclude, using Lemma [5.5] that $\operatorname{Ad}\left(g_{i}\right)\left(\Xi_{i}-[\chi, \alpha]\right) \in \Omega_{N}^{1}\left(\mathfrak{P}_{i}^{-r_{i}+1}\right)+\mathcal{I}^{\sigma}$.

Consider the identity

$\operatorname{Ad}\left(g_{i}^{\sigma}\right)\left(-\Xi_{i}+\left[\Theta_{i}^{\sigma}, \alpha\right]+\left[\chi-\chi^{\sigma}, \alpha\right]\right)=\operatorname{Ad}\left(g_{i}^{\sigma}\right)\left(-\Xi_{i}+[\chi, \alpha]\right)+\operatorname{Ad}\left(g_{i}^{\sigma}\right)\left(\left[\Theta_{i}^{\sigma}-\chi^{\sigma}, \alpha\right]\right)$.

Note that $\Theta_{i}^{\sigma}-\chi^{\sigma} \in \Omega_{N}^{1}\left(\mathfrak{g l}_{n}(\mathbb{C})\right)$ lies in $\mathcal{I}^{\sigma}$, since it is the image of $\Theta_{i}+\mathrm{p}_{\mathrm{i}}^{\mathrm{L}}(\chi)$ under the tangent map of $\sigma_{i}$. We obtain that the right hand side of (5.7) lies in $\Omega_{N}^{1}\left(\mathfrak{P}_{i}^{-r_{i}+1}\right)+\mathcal{I}^{\sigma}$ since $\Theta_{i}^{\sigma}-\chi^{\sigma} \in \Omega_{N}^{1}\left(\mathfrak{g l}_{n}(\mathbb{C})\right)$ is the image of $\Theta_{i}+\mathrm{p}_{\mathrm{i}}^{\mathrm{L}}(\chi) \in \mathcal{I}^{\sigma}$ under 
the tangent map of $\sigma_{i}$. The left hand side determines a functional $\xi_{i}^{\prime} \in \Omega_{N}^{1}\left(\mathfrak{g}_{i}^{\vee}\right)$ using the residue-trace pairing. Equation (5.2) and the second part of Lemma 5.3 show that $\xi_{i}^{\prime}$ is equal to $-\xi_{i}+\mathrm{p}_{\mathrm{i}}^{\mathrm{U}}(\chi)$. It follows that $\left(-\xi_{i}+\mathrm{p}_{\mathrm{i}}^{\mathrm{U}}(\chi)\right)\left(\mathfrak{P}_{i}^{r_{i}}\right) \subset \mathcal{I}^{\sigma}$. Since $\widetilde{W}_{r_{i}} \subset \mathfrak{P}_{i}^{r_{i}}$, we see that $\widehat{Z}_{i}+\mathfrak{P}_{i}^{r_{i}}=\mathfrak{g}_{i}$. Thus, $\left(-\xi_{i}+\mathrm{p}_{\mathrm{i}}^{\mathrm{U}}(\chi)\right)\left(\mathfrak{g}_{i}\right) \subset \mathcal{I}^{\sigma}$. Lemma 5.2 implies that both sides of (5.7) lie in $\mathcal{I}^{\sigma}$. In particular, $\Xi_{i}-[\chi, \alpha] \in \mathcal{I}^{\sigma}$.

Finally, let $\mathcal{I}^{\prime}$ be the ideal generated by $\Xi_{i}-[\chi, \alpha], \Theta_{i}+\mathrm{p}_{\mathrm{i}}^{\mathrm{L}}(\chi)$, and $d \hat{\Gamma}_{i}^{0}$. Since $-\Xi_{i}^{\mathfrak{V}}+\mathrm{p}_{\mathrm{i}}^{\mathrm{U}}(\chi)$ is the restriction of the difference of the first two generators, it lies in the ideal $\mathcal{I}^{\prime}$. Therefore, $\kappa^{\prime}=\tilde{\kappa}+\chi$ lies in $\mathcal{I}^{\prime}$. The image of $\kappa^{\prime}$ in $\Omega_{N}^{1}\left(\left(\prod_{i}\left(\mathrm{~T}\left(\widetilde{\mathcal{M}}_{i}\right)\right)\right) / \mathrm{T}_{\mathfrak{g l}_{n}}\right)$ is $\kappa$, so $\kappa \in \mathcal{I}^{\prime}$. It follows that $\mathcal{I}^{\sigma}=\mathcal{I}^{\prime}$.

We will now show that $\mathcal{I}^{\sigma}$ is the restriction of $\mathcal{I}$ to $N$.

Lemma 5.8. Let $\mathcal{J}$ be a differential ideal on $N$. Suppose that $X \in \Omega_{\widehat{\mathcal{M}}}^{1}\left(\mathfrak{P}_{i}^{1}\right)$ satisfies

$$
\tau_{\nu}(X)-\left[X, \hat{\Gamma}_{i \nu}\right] \in \mathcal{J}
$$

Then, $X \in \mathcal{J}$.

Proof. Without loss of generality, assume that $\nu=\frac{d z_{i}}{z_{i}}$ and $\tau_{\nu}=z_{i} \partial_{z_{i}}$. To simplify notation, we will suppress the subscript $i$ in the proof. Let $\psi: \Omega_{\widehat{\mathcal{M}}}^{1}\left(\mathfrak{P}^{1}\right) \rightarrow$ $\Omega_{\widehat{\mathcal{M}}}^{1}\left(\mathfrak{P}^{-r+1}\right)$ be defined by $\psi(Y)=\tau_{\nu}(Y)-\left[Y, \hat{\Gamma}_{\nu}\right]$.

First, we prove the case $r=0$, so $e=1$ and $P=\operatorname{GL}_{n}(\mathfrak{o})$. Note that $\hat{\Gamma}_{\nu} \in\left(\mathfrak{t}^{b}\right)^{\prime}$ by Definition 3.3 and $\tau_{\nu}(Y)=\ell Y$ for $Y \in \Omega_{\widehat{\mathcal{M}}}^{1}\left(z^{\ell} \mathfrak{g l}_{n}(\mathbb{C})\right)$. It follows that $\psi$ : $z^{\ell} \mathfrak{g l}_{n}(\mathbb{C}) \rightarrow z^{\ell} \mathfrak{g l}_{n}(\mathbb{C})$ is an isomorphism, since $-\ell$ is never an eigenvalue of ad $\left(\hat{\Gamma}_{\nu}\right)$. Now, suppose that $X \in \Omega_{\widehat{\mathcal{M}}}^{1}\left(\mathfrak{g}^{\ell}\right)+\mathcal{J}$ for $\ell \geq 1$, say $X \in X_{\ell}+\Omega_{\widehat{\mathcal{M}}}^{1}\left(\mathfrak{g}^{\ell+1}\right)+\mathcal{J}$ for some $X_{\ell} \in \Omega_{\widehat{\mathcal{M}}}^{1}\left(z^{\ell} \mathfrak{g l}_{n}(\mathbb{C})\right)$. (This holds trivially for $\ell=1$.) We see that the image of $\psi\left(X_{\ell}\right) \in \Omega_{\widehat{\mathcal{M}}}^{1}\left(\mathfrak{g}^{\ell+1}\right)+\mathcal{J}$. However, we have shown that $\psi$ induces an automorphism of $\Omega_{\widehat{\mathcal{M}}}^{1}\left(\mathfrak{g}^{\ell} / \mathfrak{g}^{\ell+1}\right)$, so $X_{\ell} \in \Omega_{\widehat{\mathcal{M}}}^{1}\left(\mathfrak{g}^{\ell+1}\right)+\mathcal{J}$. By induction, we conclude that $X \in \mathcal{J}$.

Let $W_{\ell}=\operatorname{ker}\left(\bar{\pi}_{\mathfrak{t}}\right) \subset \mathfrak{P}^{\ell} / \mathfrak{P}^{\ell+1}$. We define the projection $\mathrm{p}_{\mathfrak{t}}: \mathfrak{g l}_{n}(F) \rightarrow \operatorname{ker}\left(\pi_{\mathfrak{t}}\right)$ by $\mathrm{p}_{\mathfrak{t}}(X)=X-\pi_{\mathfrak{t}}(X)$, and write $\overline{\mathrm{p}}_{\mathfrak{t}}: \mathfrak{P}^{\ell} / \mathfrak{P}^{\ell+1} \rightarrow W_{\ell}$ for the induced map on cosets. Let $\psi: \Omega_{\widehat{\mathcal{M}}}^{1}\left(\mathfrak{P}^{1}\right) \rightarrow \Omega_{\widehat{\mathcal{M}}}^{1}\left(\mathfrak{P}^{-r+1}\right)$ be defined by $\psi(Y)=\tau_{\nu}(Y)-\left[Y, \hat{\Gamma}_{\nu}\right]$. Since Lemma 4.1 states that $\psi(t)=\delta_{e}(t) \in \mathfrak{t}$ for all $t \in \Omega_{\widehat{\mathcal{M}}}^{1}\left(\mathfrak{t}^{1}\right), \mathrm{p}_{\mathfrak{t}} \circ \psi(t)=0$. This implies that $\mathrm{p}_{\mathfrak{t}}(\psi(Y))=\mathrm{p}_{\mathfrak{t}}\left(\psi\left(\mathrm{p}_{\mathfrak{t}}(Y)\right)\right)$ for all $Y \in \Omega_{\widehat{\mathcal{M}}}^{1}\left(\mathfrak{P}^{\ell}\right)$.

First, we show inductively that $X^{\prime}=\mathrm{p}_{\mathfrak{t}}(X) \in \mathcal{J}$. Suppose that $X^{\prime} \in \mathcal{J}+$ $\Omega_{\widehat{\mathcal{M}}}^{1}\left(\mathfrak{P}^{\ell}\right)$, say $X^{\prime} \in X_{\ell}^{\prime}+\mathcal{J}$ with $X_{\ell}^{\prime} \in \Omega_{\widehat{\mathcal{M}}}^{1}\left(\mathfrak{P}^{\ell}\right)$. Since $\psi(X) \in \mathcal{J}$, we obtain $\mathrm{p}_{\mathfrak{t}}\left(\psi\left(X_{\ell}^{\prime}\right)\right)=\mathrm{p}_{\mathfrak{t}}(\psi(X))+\mathcal{J} \subset \mathcal{J}$. By part (5) of Proposition 2.4, the map $\bar{\psi}: W_{\ell} \rightarrow$ $W_{\ell-r}$ induced by $\mathrm{p}_{\mathfrak{t}} \circ \psi$ is an isomorphism, so $X_{\ell}^{\prime} \in \mathcal{J}+\Omega_{\widehat{\mathcal{M}}}^{1}\left(\mathfrak{P}^{\ell+1}\right)$. It follows that $X^{\prime} \in \mathcal{J}+\Omega_{\widehat{\mathcal{M}}}^{1}\left(\mathfrak{P}^{\ell+1}\right)$. Since the inductive hypothesis holds trivially for $\ell=1$, we conclude that $X^{\prime} \in \mathcal{J}$.

We now have $X \in \pi_{\mathfrak{t}}(X)+\mathcal{J}$ and also $\delta_{e}\left(\pi_{\mathfrak{t}}(X)\right)=\psi\left(\pi_{\mathfrak{t}}(X)\right)=\psi(X)-\psi\left(X^{\prime}\right) \in$ $\mathcal{J}$. Since $\delta_{e}$ is an automorphism of $\mathfrak{t}^{1}$, this gives $\pi_{\mathfrak{t}}(X) \in \mathcal{J}$, and hence $X \in \mathcal{J}$.

In the following, let $\chi$ be the section of $\left.\mathrm{T}_{\mathfrak{g l}_{n}}\right|_{N}$ constructed in Lemma 5.3.

Proposition 5.9. The following identity holds modulo $\mathcal{I}^{\sigma}$ :

$$
\Upsilon^{0}+\chi \in \operatorname{Ad}\left(\hat{g}_{i}^{-1}\right) A_{\widetilde{\mathcal{M}}, i}^{\prime}+\hat{g}_{i}^{-1} d \hat{g}_{i}+\mathcal{I}^{\sigma}
$$


Proof. By Proposition 5.7 .

$$
\tau_{\nu} \Upsilon^{0}-d \alpha-\left[\Upsilon^{0}+\chi, \alpha\right]+\Omega_{\widehat{\mathcal{M}}}^{1}\left(\mathfrak{g}_{j}^{1} \frac{d z_{j}}{z_{j} \nu}\right)=\Xi_{j}-[\chi, \alpha] \in \mathcal{I}^{\sigma} .
$$

Thus, if $\nu$ is a global meromorphic one-form on $\mathbb{P}^{1}$, we obtain $\nu \wedge\left(\tau_{\nu} \Upsilon^{0}-d \alpha-\left[\Upsilon^{0}+\chi, \alpha\right]\right) \in$ $\mathcal{I}^{\sigma}$. This follows from the observation that the principal part at each $x_{j} \in \mathbb{P}^{1}$ lies in $\mathcal{I}^{\sigma}$. Applying the interior derivative $\iota_{\tau_{\nu}}$ implies that

$$
\tau_{\nu} \Upsilon^{0}-d \alpha-\left[\Upsilon^{0}+\chi, \alpha\right] \in \mathcal{I}^{\sigma}
$$

Now, we set $\nu=\frac{d z_{i}}{z_{i}}$ and $\tau_{\nu}=z_{i} \partial_{z_{i}}$. Note that $\Upsilon^{0}-\operatorname{Ad}\left(\hat{g}_{i}^{-1}\right)\left(A_{\widetilde{\mathcal{M}}, i}^{\prime}\right) \in \Omega_{\widehat{\mathcal{M}}}^{1}\left(\mathfrak{g}_{i}\right)$ by construction. Therefore, setting $\hat{p}_{i}^{\sigma}=\hat{g}_{i}\left(g_{i}^{\sigma}\right)^{-1}$,

$$
\begin{aligned}
\operatorname{Ad}\left(g_{i}^{\sigma}\right)\left(\Upsilon^{0}\right)+\operatorname{Ad}\left(g_{i}^{\sigma}\right)(\chi)-\left(\operatorname{Ad}\left(\left(\hat{p}_{i}^{\sigma}\right)^{-1}\right)\left(A_{\widetilde{\mathcal{M}}_{i}}^{\prime}\right)+\left(d g_{i}^{\sigma}\right)\left(g_{i}^{\sigma}\right)^{-1}\right) \in \\
-\phi_{i}\left(\left(d g_{i}^{\sigma}\right)\left(g_{i}^{\sigma}\right)^{-1}+\operatorname{Ad}\left(\left(\hat{p}_{i}^{\sigma}\right)^{-1}\right)\left(A_{\widetilde{\mathcal{M}}, i}^{\prime}\right)-\operatorname{Ad}\left(g_{i}^{\sigma}\right)\left(\Upsilon^{0}\right)\right)+\operatorname{Ad}\left(g_{i}^{\sigma}\right)(\chi)+\Omega_{\widehat{\mathcal{M}}}^{1}\left(\mathfrak{P}_{i}^{1}\right)= \\
-\operatorname{Ad}\left(g_{i}^{\sigma}\right)\left(\Theta_{i}^{\sigma}-\chi\right) \in \mathcal{I}^{\sigma} .
\end{aligned}
$$

It follows that $\Upsilon^{0}+\chi \in \operatorname{Ad}\left(\hat{g}_{i}^{-1}\right) A_{\widetilde{\mathcal{M}}, i}^{\prime}+\hat{g}_{i}^{-1} d \hat{g}_{i}+\mathcal{I}^{\sigma}+\Omega_{\widehat{\mathcal{M}}^{1}}^{1}\left(\mathfrak{P}_{i}^{1}\right)$.

Let $\Upsilon^{\prime}=\operatorname{Ad}\left(\hat{g}_{i}\right)\left(\Upsilon^{0}+\chi\right)-\left(d \hat{g}_{i}\right) \hat{g}_{i}^{-1}$. From the argument above, we see that $\Upsilon^{\prime}-A_{\widetilde{\mathcal{M}}, i}^{\prime} \in \Omega_{\widehat{\mathcal{M}}}^{1}\left(\mathfrak{P}_{i}^{1}\right)+\mathcal{I}^{\sigma}$. Substituting $\operatorname{Ad}\left(\hat{g}_{i}^{-1}\right)\left(\Upsilon^{\prime}\right)+\hat{g}_{i}^{-1} d \hat{g}_{i}=\Upsilon^{0}+\chi$ and $\operatorname{Ad}\left(\hat{g}_{i}^{-1}\right) \hat{\Gamma}_{i \nu}+\hat{g}_{i}^{-1} \tau_{\nu}\left(\hat{g}_{i}\right)=\alpha$ into (5.9) , we obtain

$$
\tau_{\nu}\left(\Upsilon^{\prime}\right)-d \hat{\Gamma}_{i \nu}-\left[\Upsilon^{\prime}, \hat{\Gamma}_{i \nu}\right] \in \mathcal{I}^{\sigma}
$$

Since $d \hat{\Gamma}_{i \nu}-d \hat{\Gamma}_{i}^{0}=\tau_{\nu}\left(A_{\widetilde{\mathcal{M}}, i}^{\prime}\right)-\left[A_{\widetilde{\mathcal{M}}, i}^{\prime}, \hat{\Gamma}_{i \nu}\right]$ by $(\underline{5.3)}$, we deduce that

$$
\tau_{\nu}\left(\Upsilon^{\prime}-A_{\widetilde{\mathcal{M}}, i}^{\prime}\right)-\left[\Upsilon^{\prime}-A_{\widetilde{\mathcal{M}}, i}^{\prime}, \hat{\Gamma}_{i \nu}\right] \in d \hat{\Gamma}_{i}^{0}+\mathcal{I}^{\sigma}
$$

The left hand side lies in $\mathcal{I}^{\sigma}$, since $d \hat{\Gamma}_{i}^{0} \in \mathcal{I}^{\sigma}$. The statement of the Lemma now follows, since by Lemma $5.8, \Upsilon^{\prime}-A_{\widetilde{\mathcal{M}}, i}^{\prime} \in \mathcal{I}^{\sigma}$.

Corollary 5.10. The sections $\chi_{i}$ of $\mathrm{T}_{\mathfrak{g l}_{n}}$ satisfy $\tilde{\kappa}+\chi_{i} \in \mathcal{I}^{\sigma}$. Moreover, $\chi_{i} \in$ $\chi_{j}+\mathcal{I}^{\sigma}$ and $d \chi_{i}+\chi_{i} \wedge \chi_{i} \in \mathcal{I}^{\sigma}$.

Proof. Let $\chi$ be the section of $\mathrm{T}_{\mathfrak{g l}_{n}}$ constructed in Proposition 5.7. To prove the first statement, it suffices to show that $\chi_{i}-\chi \in \mathcal{I}^{\sigma}$. However, for any $i \in I$,

$$
\chi_{i}-\chi=\phi_{i}\left(\operatorname{Ad}\left(\hat{g}_{i}^{-1}\right) A_{\widetilde{\mathcal{M}}, i_{i}}^{\prime}+\hat{g}_{i}^{-1} d \hat{g}_{i}-\Upsilon^{0}-\chi\right) \in \mathcal{I}^{\sigma}
$$

by Proposition 5.9 .

In order to prove the second part, we recall that $\Upsilon^{0}$ is defined as $\varsigma\left(\left(\bar{\Phi}_{j}\right)_{j \in I}\right)$. It follows that $d \Upsilon^{0}=\varsigma\left(\left(d \bar{\Phi}_{j}\right)_{j \in I}\right)$. By construction, $\bar{\Phi}_{j}=\operatorname{Ad}\left(\hat{g}_{j}^{-1}\right)\left(A_{\widetilde{\mathcal{M}}, j}\right)$. Therefore, $d \bar{\Phi}_{j} \in-\left[\operatorname{Ad}\left(\hat{g}_{j}^{-1}\right) A_{\widetilde{\mathcal{M}}, j}, \hat{g}_{j}^{-1} d \hat{g}_{j}\right]+\Omega_{N}^{1}\left(\mathfrak{g}_{j}\right)$ since $d A_{\widetilde{\mathcal{M}}, i}=0$.

By Proposition [5.9] $\operatorname{Ad}\left(\hat{g}_{j}^{-1}\right) A_{\widetilde{\mathcal{M}}, j}+\hat{g}_{j}^{-1} d \hat{g}_{j} \in \Upsilon^{0}+\chi_{i}+\mathcal{I}^{\sigma}$. We conclude that

$$
\left[\operatorname{Ad}\left(\hat{g}_{j}^{-1}\right) A_{\widetilde{\mathcal{M}}, j}, \hat{g}_{j}^{-1} d \hat{g}_{j}\right] \in-\left(\Upsilon^{0}+\chi_{i}\right) \wedge\left(\Upsilon^{0}+\chi_{i}\right)+\mathcal{I}^{\sigma}+\Omega_{N}^{2}\left(\mathfrak{g}_{j}\right),
$$

and moreover $\varsigma\left(\left(d \bar{\Phi}_{j}\right)_{j \in I}\right) \in-\varsigma\left(\left(\left(\Upsilon^{0}+\chi_{i}\right) \wedge\left(\Upsilon^{0}+\chi_{i}\right)\right)_{j \in I}\right)+\mathcal{I}^{\sigma}$. Thus, by Lemma4.10. $\left(\Upsilon^{0}+\chi_{i}\right) \wedge\left(\Upsilon^{0}+\chi_{i}\right)+\varsigma\left(\left(d \bar{\Phi}_{j}\right)_{j \in I}\right) \in \chi_{i} \wedge \chi_{I}+\mathcal{I}^{\sigma}$. 
Finally,

$$
\begin{aligned}
& d\left(\operatorname{Ad}\left(\hat{g}_{i}^{-1}\right) A_{\widetilde{\mathcal{M}}, i}+\hat{g}_{i}^{-1} d \hat{g}_{i}\right)= \\
&-\left(\operatorname{Ad}\left(\hat{g}_{i}^{-1}\right) A_{\widetilde{\mathcal{M}}, i}+\hat{g}_{i}^{-1} d \hat{g}_{i}\right) \wedge\left(\operatorname{Ad}\left(\hat{g}_{i}^{-1}\right) A_{\widetilde{\mathcal{M}}, i}+\hat{g}_{i}^{-1} d \hat{g}_{i}\right) \\
& \quad \in-\left(\Upsilon^{0}+\chi_{i}\right) \wedge\left(\Upsilon^{0}+\chi_{i}\right)+\mathcal{I}^{\sigma} .
\end{aligned}
$$

It follows that

$$
\begin{aligned}
d \chi_{i}+\chi_{i} \wedge \chi_{i} & =\phi_{i}\left(d\left(\operatorname{Ad}\left(\hat{g}_{i}^{-1}\right) A_{\widetilde{\mathcal{M}}, i}^{\prime}+\hat{g}_{i}^{-1} d \hat{g}_{i}-\Upsilon^{0}\right)\right)+\chi_{i} \wedge \chi_{i} \\
& \in \phi_{i}\left(-\left(\Upsilon^{0}+\chi_{i}\right) \wedge\left(\Upsilon^{0}+\chi_{i}\right)+\left(\Upsilon^{0}+\chi_{i}\right) \wedge\left(\Upsilon^{0}+\chi_{i}\right)-\chi_{i} \wedge \chi_{i}\right)+\chi_{i} \wedge \chi_{i}+\mathcal{I}^{\sigma} \\
& =\mathcal{I}^{\sigma} .
\end{aligned}
$$

Corollary 5.11. Fix $j \in I$. The ideal $\mathcal{I}^{\sigma}$ is generated by $\Xi_{i}-\left[\chi_{j}, \alpha\right], \Theta_{i}+\mathrm{p}_{\mathrm{i}}^{\mathrm{L}}\left(\chi_{j}\right)$, and $d \hat{\Gamma}_{i}^{0}$ for all $i \in I$. In particular, $\mathcal{I}^{\sigma}$ is the restriction of $\mathcal{I}$ to $N$, and $\mathcal{I}$ does not depend on the choice of $j$.

Proof. By Corollary 5.10, $\chi_{j}$ satisfies the the conditions of Proposition 5.7 The same proposition implies that $\mathcal{I}^{\sigma}$ has the given generators.

5.3. Invariance. In this section, we will show that $\mathcal{I}$ is the pullback of a differential ideal $\overline{\mathcal{I}}$ on $\widetilde{\mathcal{M}}(\mathbf{P}, \mathbf{r}, \mathbf{x})$. Moreover, the ideal $\overline{\mathcal{I}}$ is Pfaffian and integrable.

Proposition 5.12. Let $X$ be a section of $\mathrm{T}_{\mathfrak{g l}_{n}}$, and take $\omega \in \mathcal{I}$. Then, $\iota_{X} \omega \in \mathcal{I}$. In particular, if $\omega \in \mathcal{I} \cap \Omega_{\widehat{\mathcal{M}}}^{1}$, then $\iota_{X} \omega=0$.

Proof. It suffices to show that the generators of $\mathcal{I}$ are in the kernel of $\iota_{X}$. Since $\hat{\Gamma}_{i}$ is invariant under the action of $\mathrm{GL}_{n}(\mathbb{C})$, we see that $\iota_{X} d \hat{\Gamma}_{i}^{0}=0$ and $\iota_{X}\left(A_{\widetilde{\mathcal{M}}, i}\right)=0$. It follows that any term involving $A_{\widetilde{\mathcal{M}}, i}$ lies in the kernel of $\iota_{X}$. We now calculate $\iota_{X}\left(\Xi_{i}-\left[\chi_{j}, \alpha\right]\right)$. By the argument above,

$$
\iota_{X}\left(\Xi_{i}\right)=\iota_{X}(-d \alpha)=-[X, \alpha] .
$$

On the other hand,

$$
\iota_{X}\left(\chi_{j}\right)=\iota_{X} \phi_{j}\left(\hat{g}_{j}^{-1} d \hat{g}_{j}\right)=-X .
$$

It follows that $\iota_{X}\left(\Xi_{i}-\left[\chi_{j}, \alpha\right]\right)=-[X, \alpha]+[X, \alpha]=0$. On the other hand,

$$
\iota_{X}\left(\Theta_{i}\right)=\iota_{X}\left(\left(d g_{i}\right) g_{i}^{-1}+\Omega_{\widehat{\mathcal{M}}}^{1}\left(\mathfrak{u}_{i}\right)\right)=\operatorname{Ad}\left(g_{i}\right)(X)+\mathfrak{u}_{i} .
$$

Using (5.10), we see that $\iota_{X}\left(\Theta_{i}+\mathrm{p}_{\mathrm{i}}^{\mathrm{L}}(X)\right)=\operatorname{Ad}\left(g_{i}\right)(X)-\operatorname{Ad}\left(g_{i}\right)(X)+\mathfrak{u}_{i}=\mathfrak{u}_{i}$.

We now show that the ideal $\mathcal{I}$ is $\mathrm{GL}_{n}(\mathbb{C})$-invariant.

Proposition 5.13. Let $g: V \rightarrow \mathrm{GL}_{n}(\mathbb{C})$ be a function defined on a neighborhood $V \subset \widehat{\mathcal{M}}$, and let $\hat{\rho}_{g}: V \rightarrow \widehat{\mathcal{M}}$ be the map determined by the action of $g$. Then, $\hat{\rho}_{g}^{*} \mathcal{I}=\mathcal{I}$. Moreover, $\hat{\rho}_{g}^{*}\left(\Xi_{i}-\left[\chi_{j}, \alpha\right]\right)=\operatorname{Ad}(g)\left(\Xi_{i}-\left[\chi_{j}, \alpha\right]\right)$ and $\hat{\rho}_{g}^{*}\left(\Theta_{i}+\mathrm{p}_{\mathrm{i}}^{\mathrm{L}}\left(\chi_{j}\right)\right)=$ $\left.\Theta_{i}+\mathrm{p}_{\mathrm{i}}^{\mathrm{L}}\left(\chi_{j}\right)\right)$. 
Proof. First, we calculate $\hat{\rho}_{g}^{*}\left(\chi_{j}\right)$. One easily checks that

$$
\hat{\rho}_{g}^{*}\left(\chi_{j}\right)=\operatorname{Ad}(g) \chi_{j}-(d g) g^{-1} \text {. }
$$

We also see that $\hat{\rho}_{g}^{*}\left(\Xi_{i}\right)=\operatorname{Ad}(g)\left(\Xi_{i}\right)-\left[(d g) g^{-1}, \alpha\right]$ and $\hat{\rho}_{g}^{*}\left(\Theta_{i}\right)=-\operatorname{Ad}\left(g_{i} g^{-1}\right)\left((d g) g^{-1}\right)+$ $\Theta_{i}$. We see that $\hat{\rho}_{g}^{*}\left(\Xi_{i}-\left[\chi_{j}, \alpha\right]\right)=\operatorname{Ad}(g)\left(\Xi_{i}-\left[\chi_{j}, \alpha\right]\right) \in \mathcal{I}$ and $\hat{\rho}_{g}^{*}\left(\Theta_{i}+\mathrm{p}_{\mathrm{i}}^{\mathrm{L}}\left(\chi_{j}\right)\right)=$ $\Theta_{i}+\mathrm{p}_{\mathrm{i}}^{\mathrm{L}}\left(\chi_{j}\right) \in \mathcal{I}$. Finally, $\hat{\rho}_{g}^{*} d \hat{\Gamma}_{i}^{0}=d \hat{\Gamma}_{i}^{0}$. It follows that $\hat{\rho}_{g}^{*}(\mathcal{I}) \subset \mathcal{I}$.

In the following, $\widetilde{\mathcal{M}}=\widetilde{\mathcal{M}}(\mathbf{P}, \mathbf{x}, \mathbf{r})$ and $q: \widehat{\mathcal{M}} \rightarrow \widetilde{\mathcal{M}}$ is the natural projection.

Corollary 5.14. There exists a differential ideal $\overline{\mathcal{I}}$ on $\widetilde{\mathcal{M}}(\mathbf{P}, \mathbf{x}, \mathbf{r})$ such that $q^{*} \overline{\mathcal{I}}=$ $\mathcal{I}$.

Proof. Since $\widehat{\mathcal{M}}$ is a principal $\mathrm{GL}_{n}(\mathbb{C})$-bundle over $\widetilde{\mathcal{M}}$, we may choose a cover of $\widetilde{\mathcal{M}}$ by neighborhoods $W$ with the property that $q^{-1}(W) \cong \mathrm{GL}_{n}(\mathbb{C}) \times W$. Define $\Theta_{i}^{G}$ and $\Xi_{i}^{G}$ on $q^{-1}(W)$ by $\Theta_{i}^{G}(g, w)=\Theta_{i}+p_{i}^{\mathrm{L}}\left(\chi_{j}\right)+\Omega_{\widehat{\mathcal{M}}}^{1}\left(\mathfrak{u}_{i}\right)$ and $\Xi_{i}^{G}(g, w)=$ $\operatorname{Ad}\left(g^{-1}\right)\left(\Xi_{i}-\left[\chi_{j}, \alpha\right]\right)$. It is clear that the collection $\Theta_{i}^{G}, \Xi_{i}^{G}$, and $d \hat{\Gamma}_{i}^{0}$ over all $i \in I$ generates $\left.\mathcal{I}\right|_{q^{-1}(W)}$. By Proposition 5.13, $\hat{\rho}_{h}^{*}\left(\Theta_{i}^{G}\right)=\Theta_{i}^{G}$ and $\hat{\rho}_{h}^{*}\left(\Xi_{i}^{G}\right)=\Xi_{i}^{G}$.

Proposition 5.12 shows that each of the generators determines a well-defined form on $q^{*} T(W) \cong T\left(q^{-1}(W)\right) / \mathfrak{g l}_{n}(\mathbb{C})$. By invariance, $\Theta_{i}^{G}, \Xi_{i}^{G}$, and $d \hat{\Gamma}_{i}^{0}$ descend to differential forms on $T(\widetilde{\mathcal{M}})$. These generate the ideal $\overline{\mathcal{I}}$.

\section{INTEGRABILITY}

We will now show that $\mathcal{I}$ is an integrable Pfaffian system in the sense of [7, II.2.4]. In general, if $\mathcal{J}$ is a differential ideal on a smooth variety $X$, we write $\mathcal{J}^{1}=\mathcal{J} \cap \Omega_{X}^{1}$. It suffices to show that the coherent sheaf $\mathcal{I}^{1}$ is locally free and that $d \mathcal{I} \subset \mathcal{I}$.

Let $\mathcal{J}$ be the ideal on $\widehat{\mathcal{M}}$ generated by $\Xi_{i}, d \hat{\Gamma}_{i}^{0}$, and $\Theta_{i}$ for all $i \in I$. Note that $\mathcal{I} \subset \mathcal{J}$. We will first show that $\mathcal{J}$ is a Pfaffian system and that $\mathcal{I}^{1} \subset \mathcal{J}^{1}$ has constant corank.

We need to describe a (local) minimal basis for $\mathcal{J}$. Since $\mathcal{J}$ is independent of the choice of one-form $\nu$, we may assume that $\nu=\frac{d z_{i}}{z_{i}}$ when working locally at $x_{i}$. As in Section 5.2, we will restrict to sufficiently small neighborhoods $N_{i} \subset U_{i} \backslash \mathrm{GL}_{n}(\mathbb{C})$ and the corresponding neighborhood $N \subset \widehat{\mathcal{M}}$.

By part (2) of Proposition 2.4 the map $\pi_{\mathfrak{t}_{i}}$ induces a map $\pi_{\mathfrak{t}_{i}}^{\prime}: \mathfrak{P}_{i} / \mathfrak{P}_{i}^{r_{i}} \rightarrow \mathfrak{t}_{i} / \mathfrak{t}_{i}^{r_{i}}$. Choose a vector space lift $Z_{i}^{\text {od }} \subset \mathfrak{P}_{i}$ of $\operatorname{ker}\left(\pi_{\mathfrak{t}_{i}}^{\prime}\right)$ and a lift $Z_{i}^{\mathfrak{u}} \subset \mathfrak{g}_{i}$ of $\mathfrak{g}_{i} / \mathfrak{P}_{i}$. Note that these vector spaces are trivial when $r=0$. We define locally the 'off-diagonal' and $\mathfrak{u}$ components of $\Xi_{i}$ to be $\Xi_{i}^{\text {od }}=\left.\xi_{i}\right|_{Z^{\text {od }}} \in \Omega_{N}^{1}\left(\left(Z^{\text {od }}\right)^{\vee}\right)$ and $\Xi_{i}^{\mathfrak{u}}=\left.\xi_{i}\right|_{Z_{i}^{\mathfrak{u}}} \in \Omega_{N}^{1}\left(\left(Z_{i}^{\mathfrak{u}}\right)^{\vee}\right)$ respectively.

Proposition 6.1. The ideal $\left.\mathcal{J}\right|_{N}$ is generated by $\Theta_{i}, \Xi_{i}^{o d}, \Xi_{i}^{\mathfrak{u}}$, and $d \hat{\Gamma}_{i}^{0}$ for $i \in I$.

Proof. As above, we assume that $\nu=\frac{d z_{i}}{z_{i}}$. By Lemma [5.2, it suffices to show that $\xi_{i}\left(\mathfrak{g}_{i}\right)$ lies in the ideal $\mathcal{J}^{\prime}$ generated by $d \hat{\Gamma}_{i}^{0}, \Theta_{i}, \Xi_{i}^{\text {od }}$, and $\Xi_{i}^{\mathfrak{u}}$, since $\mathcal{J}^{\prime} \subset \mathcal{J}$.

Let $\mathcal{J}_{1}$ be the ideal generated by $\Theta_{i}$ and $d \hat{\Gamma}_{i}^{0}$ for each $i \in I$. By Lemma 5.6 (applied with $\chi=0), \Upsilon^{0} \in \operatorname{Ad}\left(\hat{g}_{i}^{-1}\right) A_{\widetilde{\mathcal{M}}_{, i}}^{\prime}+\hat{g}_{i}^{-1} d \hat{g}_{i}+\Omega_{W}^{1}\left(\left(\mathfrak{P}_{i}^{1}\right)^{g_{i}}\right)+\mathcal{J}_{1}$. Lemma 5.5 states that

$$
\operatorname{Ad}\left(g_{i}^{\sigma}\right) \Xi_{i} \in \Omega_{W}^{1}\left(\left[\mathfrak{P}_{i}^{1}, \operatorname{Ad}\left(g_{i}^{\sigma}\right)(\alpha)\right]+\mathfrak{P}_{i}^{1}\right)+\mathcal{J}_{1} \subset \Omega_{W}^{1}\left(\mathfrak{P}^{-r+1}\right)+\mathcal{J}_{1} .
$$


Therefore, $\xi_{i}\left(\mathfrak{P}_{i}^{r}\right) \subset \mathcal{J}_{1}$.

We will now show that $\operatorname{Ad}\left(g_{i}^{\sigma}\right)\left(\Xi_{i}\right) \in \mathcal{J}^{\prime}+\Omega_{N}^{1}\left(\mathfrak{P}_{i}^{1}\right)$. Take $1 \leq j \leq r$, and assume inductively that there exists $X \in \Omega_{N}^{1}\left(\mathfrak{P}_{i}^{j}\right)$ such that

$$
\operatorname{Ad}\left(g_{i}^{\sigma}\right) \Xi_{i} \in\left[X, \operatorname{Ad}\left(g_{i}^{\sigma}\right)(\alpha)\right]+\mathcal{J}^{\prime}+\Omega_{N}^{1}\left(\mathfrak{P}_{i}^{1}\right) .
$$

Part (3) of Proposition 2.4 shows that $\left[X, \operatorname{Ad}\left(g_{i}^{\sigma}\right)(\alpha)\right]+\Omega_{N}^{1}\left(\mathfrak{P}_{i}^{j-r+1}\right) \in \operatorname{ker}\left(\bar{\pi}_{\mathfrak{t}_{i}}\right)$. Thus, by part (4) of the same proposition, $\left\langle\left[X, \operatorname{Ad}\left(g_{i}^{\sigma}\right)(\alpha)\right], \mathfrak{t}_{i}^{r-j}\right\rangle_{\nu}=\{0\}$. Since $\Xi_{i}^{o d} \in \mathcal{J}^{\prime},\left\langle\left[X, \operatorname{Ad}\left(g_{i}^{\sigma}\right)(\alpha)\right], Y\right\rangle_{\nu} \in \mathcal{J}^{\prime}$ for $Y \in \operatorname{ker}\left(\pi_{\mathfrak{t}_{i}}\right) \cap \mathfrak{P}^{r-j}$. Combining these two facts gives $\left\langle\left[X, \operatorname{Ad}\left(g_{i}^{\sigma}\right)(\alpha)\right], \mathfrak{P}^{r-j}\right\rangle_{\nu} \in \mathcal{J}^{\prime}$, and we conclude that $\left[X, \operatorname{Ad}\left(g_{i}^{\sigma}\right)(\alpha)\right] \in$ $\Omega_{N}^{1}\left(\mathfrak{P}_{i}^{j-r+1}\right)+\mathcal{J}^{\prime}$. Part (5) of Proposition 2.4 now implies that $X \in \pi_{\mathfrak{t}}(X)+$ $\Omega_{N}^{1}\left(\mathfrak{P}_{i}^{j+1}\right)+\mathcal{J}^{\prime}$. Finally, since there exists $p \in P_{i}^{1}$ such that $\operatorname{Ad}(p)\left(\operatorname{Ad}\left(g_{i}^{\sigma}\right)(\alpha)\right) \in$ $\mathfrak{t}_{i}^{-r}+\mathfrak{P}_{i}^{1}$, we see that $X-\operatorname{Ad}\left(p^{-1}\right)\left(\pi_{\mathfrak{t}}(X)\right) \in \Omega_{N}^{1}\left(\mathfrak{P}_{i}^{j+1}\right)+\mathcal{J}^{\prime}$ satisfies (6.2) for $j+1$. By induction, we obtain (6.2) for $r+1$. This gives $\operatorname{Ad}\left(g_{i}^{\sigma}\right)\left(\Xi_{i}\right) \in \mathcal{J}^{\prime}+\Omega_{N}^{1}\left(\mathfrak{P}_{i}^{1}\right)$ and hence $\xi_{i}\left(\mathfrak{P}_{i}\right) \subset \mathcal{J}^{\prime}$. Finally, since $\mathfrak{g}_{i}=Z_{i}^{\mathfrak{u}}+\mathfrak{P}_{i}$, we see that $\xi_{i}\left(\mathfrak{g}_{i}\right) \subset \mathcal{I}^{\prime}$ as desired.

In the following, let $\iota: \widehat{\mathcal{M}} \rightarrow \prod_{i \in I} \widetilde{\mathcal{M}}_{i}$ be the inclusion and $T^{*} \iota: \iota^{*}\left(\Omega_{\prod_{i \in I}^{1}} \widetilde{\mathcal{M}}_{i}\right) \rightarrow$ $\Omega_{\widehat{M}}^{1}$ the induced bundle map. The generators of $\mathcal{I}$ (resp. $\mathcal{J}$ ) lift to a set of generators for a differential ideal $\widetilde{\mathcal{I}} \subset \iota^{*} \Omega_{\prod_{i \in I}^{*} \widetilde{\mathcal{M}}_{i}}$ (resp. $\widetilde{\mathcal{J}}$ ) on $\widehat{\mathcal{M}}$. Only the lift $\tilde{\Xi}_{i}$ of $\Xi_{i}$ requires explanation. Suppose that $m=\left(\left(U_{j} g_{j}, \alpha_{j}\right)_{j \in I}\right) \in \widehat{\mathcal{M}}$. We write $\alpha_{i \nu}$ for a representative of $\alpha$ in $\left(\mathfrak{g}_{i}^{-r_{i}^{\prime}} \frac{d z_{i}}{z_{i} \nu}\right) /\left(\mathfrak{g}_{i}^{1} \frac{d z_{i}}{z_{i} \nu}\right)$. Therefore, if $m \in \widehat{\mathcal{M}}$, $\alpha_{i \nu}=\alpha+\mathfrak{g}_{i}^{1} \frac{d z_{i}}{z_{i} \nu}$. We set

$$
\tilde{\Xi}_{i}=\tau_{\nu} \Upsilon^{0}-d \alpha_{i \nu}-\left[\Upsilon^{0}, \alpha_{i \nu}\right] .
$$

It is clear that $T^{*} \iota\left(\tilde{\Xi}_{i}\right)=\Xi_{i}$. All other forms involved in the definitions of $\mathcal{I}$ and $\mathcal{J}$ are restrictions of forms on $\prod_{i \in I} \widetilde{\mathcal{M}}_{i}$, and we will not make any notational distinction between such a form and its restriction to $\widehat{\mathcal{M}}$.

Lemma 6.2. Let $\mu$ be the moment map for the action of $\mathrm{GL}_{n}(\mathbb{C})$ on $\prod_{i \in I} \widetilde{\mathcal{M}}\left(P_{i}, r_{i}\right)$. Then, $d \mu \subset \widetilde{\mathcal{I}}$.

Proof. Let $\nu$ be the global choice of one form. By construction, $\mu\left(\left(U_{i} g_{i}, \alpha_{i}\right)_{i \in I}\right)=$ $\sum_{i \in I} \operatorname{res}\left(\alpha_{i}\right)$. Let $\chi=\chi_{j}$, so $\tilde{\Xi}_{i}-[\chi, \alpha]$ is one of the generators of $\widetilde{\mathcal{I}}$. We have

$$
\begin{aligned}
\sum_{i \in I} \operatorname{Res}_{i}\left(\nu \wedge\left(\tilde{\Xi}_{i}-[\chi, \alpha]\right)\right. & =\sum_{i \in I} \operatorname{Res}_{i}\left(\nu \wedge \tau_{\nu}\left(\Upsilon^{0}\right)\right)-\operatorname{Res}_{i}\left(\nu \wedge d \alpha_{i \nu}\right)-\operatorname{Res}_{i}\left(\nu \wedge\left[\Upsilon^{0}+\chi, \alpha\right]\right) \\
& =\sum_{i \in I} \operatorname{Res}_{i}\left(\nu \wedge d \alpha_{i \nu}\right) .
\end{aligned}
$$

Here, $\sum_{i \in I} \operatorname{Res}_{i}\left(\nu \wedge \tau_{\nu}\left(\Upsilon^{0}\right)\right)$ and $\sum_{i \in I} \operatorname{Res}_{i}\left(\nu \wedge\left[\Upsilon^{0}, \alpha\right]\right)$ vanish by the residue theorem. Since $\operatorname{Res}_{i}\left(\nu \wedge \alpha_{i \nu}\right)=\operatorname{res}_{i}\left(\alpha_{i}\right)$, we see that $d \mu=\sum_{i \in I} \operatorname{Res}_{i}\left(\nu \wedge d \alpha_{i \nu}\right)$ lies in $\mathcal{I}$.

Let $\widehat{\mathcal{M}}(\mathbf{A})=q^{-1}(\widetilde{\mathcal{M}}(\mathbf{A}))$ and $N(\mathbf{A})=N \cap \widehat{\mathcal{M}}(\mathbf{A})$. We also set $\bar{N}=\prod_{i \in I}\left(\tilde{\psi}_{i}\right)^{-1}\left(N_{i}\right) \subset$ $\prod_{i \in I} \widetilde{\mathcal{M}}_{i}$ and $\bar{N}(\mathbf{A})=\bar{N} \cap\left(\prod_{i \in I} \widetilde{\mathcal{M}}\left(A_{i}\right)\right)$.

Proposition 6.3. The ideal $\mathcal{J}$ is a Pfaffian system. Moreover, the restriction map taking $\mathcal{J}^{1} \subset \Omega_{\widehat{\mathcal{M}}}^{1}$ to $\Omega_{\widehat{\mathcal{M}}(\mathbf{A})}^{1}$ is surjective. 
Proof. This is a local statement, so we will work with the restriction of $\mathcal{J}$ to $N$. Fix a point $m=\left(U_{i} g_{i}, \alpha_{i}\right)_{i \in I} \in N(\mathbf{A})$. We will first show that the restriction of $\widetilde{\mathcal{J}}^{1}$ to $\bar{N}(\mathbf{A})$ spans $\Omega \frac{1}{N(\mathbf{A})}$.

There are isomorphisms $\bar{N} \cong \prod_{i}\left(\mathfrak{P}_{i} / \mathfrak{P}_{r+1}\right)_{\text {reg }}^{\vee} \times N_{i}$ and $\bar{N}(\mathbf{A}) \cong \prod_{i \in I}\left(\pi_{\mathfrak{P}^{1}}\right)^{-1}\left(\mathcal{O}_{i}^{1}\right) \times$ $N_{i}$. Restricting $\widetilde{\mathcal{J}}$ to $\bar{N}(\mathbf{A})$, we see that all terms in $\Theta_{i}$ and $\tilde{\Xi}_{i}$ involving $A_{\widetilde{\mathcal{M}}, i}$ vanish. In particular, $\Upsilon^{0}$ and $\Phi_{i}$ vanish, so $\Theta_{i}$ becomes $\left(d g_{i}\right) g_{i}^{-1}+\Omega \frac{1}{N(\mathbf{A})}\left(\mathfrak{u}_{i}\right)$, and $\tilde{\Xi}_{i}$ is simply $d \alpha+\Omega \frac{1}{N(\mathbf{A})}\left(\mathfrak{g}_{i}^{1} \frac{d z_{i}}{z_{i} \nu}\right)$. Write $\operatorname{Ad}\left(g_{i}\right)(\alpha)=v \in\left(\pi_{\mathfrak{P}^{1}}\right)^{-1}\left(\mathcal{O}_{i}^{1}\right)$. Since $d \alpha=\operatorname{Ad}\left(g_{i}^{-1}\right)\left(d v-\left[\left(d g_{i}\right) g_{i}^{-1}, v\right]\right)$, it is easily checked that the coefficients of $\left(d g_{i}\right) g_{i}^{-1}$ and $d \alpha$ span $T_{m}^{*}(\bar{N}(\mathbf{A}))$.

On the other hand, a calculation using [2, Lemma 3.17] shows that $\operatorname{dim} T_{m}^{*}(\bar{N}(\mathbf{A}))=$ $\operatorname{dim} \mathfrak{g l}_{n}(\mathbb{C}) / \mathfrak{u}+\operatorname{dim} \mathfrak{g} / \mathfrak{P}^{r}-\operatorname{dim} \mathfrak{t}^{1} / \mathfrak{t}^{r}$, and so the coefficients of the set of generators for $\widetilde{\mathcal{J}}$ in Proposition 6.1 give a basis for $T_{m}^{*}(\bar{N}(\mathbf{A}))$. Thus, $\widetilde{\mathcal{J}}$ is a rank $\sum_{i \in I} \operatorname{dim}\left(\widetilde{\mathcal{M}}\left(A_{i}\right)\right)$ Pfaffian system.

Lemma 6.2 shows that $d \mu \in \widetilde{\mathcal{J}}$. Since the coefficients of $d \mu$ span the conormal bundle of $\widehat{\mathcal{M}}$ in $\prod_{i \in I} \widetilde{\mathcal{M}}\left(P_{i}, r_{i}\right)$, we see that the pullback of $\widetilde{\mathcal{J}}$ in $\Omega_{\widehat{\mathcal{M}}}^{*}$, i.e., $\mathcal{J}$, is a rank $\left(\sum_{i \in I} \operatorname{dim}\left(\widetilde{\mathcal{M}}\left(A_{i}\right)\right)\right)-n^{2}$ Pfaffian system. Moreover, the restriction map taking $\mathcal{J}^{1}$ to $\Omega_{N(\mathbf{A})}^{1}$ induced by the map $\widetilde{\mathcal{J}}^{1} \rightarrow \Omega \frac{1}{N(\mathbf{A})}$ is still surjective.

Lemma 6.4. Fix $j \in I$. The ideal $\mathcal{J}$ is generated by $\mathcal{I}$ and the coefficients of $\chi_{j}$.

Proof. We know that $\mathcal{I} \subset \mathcal{J}$. By Corollary $5.10, \tilde{\kappa}+\chi_{j} \in \mathcal{I}$. However, by construction, $\tilde{\kappa} \in \mathcal{J}$; it follows that $\chi_{j} \in \mathcal{J}$.

In order to prove the reverse inclusion, Proposition [5.7 shows that $\Xi_{i}-\left[\chi_{j}, \alpha\right] \in \mathcal{I}$. By assumption, $\Theta_{i}+\mathrm{p}_{\mathrm{i}}^{\mathrm{L}}\left(\chi_{j}\right) \in \mathcal{I}$ as well. It follows that $\Xi_{i}$ and $\Theta_{i}$ lie in the ideal generated by $\mathcal{I}$ and $\chi_{j}$.

There is a natural restriction map $\Omega_{\widehat{M}}^{1} \rightarrow\left(\mathrm{T}_{\mathfrak{g l}_{n}}\right)^{\vee}$. We denote by $F$ the induced $\operatorname{map} F: \mathcal{J}^{1} \rightarrow\left(\mathrm{T}_{\mathfrak{g l}_{n}}\right)^{\vee}$.

Proposition 6.5. The map $F$ is surjective, and $\mathcal{I}^{1}$ is the kernel of $F$. Moreover, $\mathcal{I}$ is a Pfaffian system.

Proof. First, we prove surjectivity. For a point $m \in \widehat{\mathcal{M}}(\mathbf{A})$, the map $F$ factors through restriction to $T\left((\widehat{\mathcal{M}}(\mathbf{A}))\right.$, since $\widehat{\mathcal{M}}(\mathbf{A})$ is $\mathrm{GL}_{n}(\mathbb{C})$-invariant. By Proposition 6.3, the restriction map $\mathcal{J}^{1} \rightarrow \Omega_{\widehat{\mathcal{M}}(\mathbf{A})}^{1}$ is a surjective bundle map. Since the map $\Omega_{\widehat{\mathcal{M}}(\mathbf{A})}^{1} \rightarrow \mathrm{T}_{\mathfrak{g l}_{n}}^{\vee}$ is surjective, it follows that $F$ is surjective.

By Proposition 5.12, $\mathcal{I}^{1} \subset \mathrm{T}_{\mathfrak{g l}_{n}}^{\perp}$. We conclude that $\mathcal{I} \subset \operatorname{ker}(F)$. Finally, since $\mathcal{I}^{1}$ and $\chi_{j}$ generate $\mathcal{J}^{1}$, the rank of $\mathcal{I}^{1}$ must be at least $\operatorname{rank}\left(\mathcal{J}^{1}\right)-n^{2}$. The rank of the kernel of $F$ is $\operatorname{rank}\left(\mathcal{J}^{1}\right)-n^{2}$, so $\mathcal{I}^{1}=\operatorname{ker}(F)$. Therefore, $\mathcal{I}$ has constant rank and is thus a Pfaffian system.

We will now prove that $\mathcal{I}$ satisfies the Frobenius integrability condition.

Lemma 6.6. Set $\chi=\chi_{j}$ as above. Then,

$$
d\left(\Upsilon^{0}+\chi\right)+\left(\Upsilon^{0}+\chi\right) \wedge\left(\Upsilon^{0}+\chi\right) \in \mathcal{I}
$$


Proof. First, we will show that

$$
d\left(\Upsilon^{0}\right) \in-\left(\Upsilon^{0}+\chi\right) \wedge\left(\Upsilon^{0}+\chi\right)+\Omega_{\widehat{\mathcal{M}}}^{2}\left(\mathfrak{g}_{i}\right)+\mathcal{I}
$$

By construction, $\Upsilon^{0} \in \operatorname{Ad}\left(\hat{g}_{i}^{-1}\left(A_{\widetilde{\mathcal{M}}, i}^{\prime}\right)\right)+\Omega_{\widehat{\mathcal{M}}}^{1}\left(\mathfrak{g}_{i}\right)$. Therefore,

$$
d\left(\Upsilon^{0}\right) \in-\left[\operatorname{Ad}\left(\hat{g}_{i}^{-1}\right)\left(A_{\widetilde{\mathcal{M}}, i}^{\prime}\right), \hat{g}_{i}^{-1} d \hat{g}_{i}\right]+\Omega_{\widehat{\mathcal{M}}}^{2}\left(\mathfrak{g}_{i}\right) .
$$

On the other hand, by Proposition 5.9,

$$
\begin{aligned}
& \left(\Upsilon^{0}+\chi\right) \wedge\left(\Upsilon^{0}+\chi\right) \in \\
& \quad\left(\operatorname{Ad}\left(\hat{g}_{i}^{-1}\right) A_{\widetilde{\mathcal{M}}, i}^{\prime}+\hat{g}_{i}^{-1} d \hat{g}_{i}\right) \wedge\left(\operatorname{Ad}\left(\hat{g}_{i}^{-1}\right) A_{\widetilde{\mathcal{M}}, i}^{\prime}+\hat{g}_{i}^{-1} d \hat{g}_{i}\right)+\Omega_{\widehat{\mathcal{M}}}^{2}\left(\mathfrak{g}_{i}\right)+\mathcal{I} .
\end{aligned}
$$

The right hand side is equivalent to $\left[\operatorname{Ad}\left(\hat{g}_{i}^{-1}\right) A_{\widetilde{\mathcal{M}}, i}^{\prime}, \hat{g}_{i}^{-1} d \hat{g}_{i}\right]+\Omega_{\widehat{\mathcal{M}}}^{2}\left(\mathfrak{g}_{i}\right)+\mathcal{I}$. This proves (6.3).

It suffices to show that

$$
\left(\Upsilon^{0}+\chi\right) \wedge\left(\Upsilon^{0}+\chi\right)=\varsigma\left(\left(\left(\Upsilon^{0}+\chi\right) \wedge\left(\Upsilon^{0}+\chi\right)+\Omega_{\widehat{\mathcal{M}}}^{2}\left(\mathfrak{g}_{i}\right)\right)_{i \in I}\right)+\chi \wedge \chi,
$$

since the right hand side is equal to $-d\left(\Upsilon^{0}+\chi\right)(\bmod \mathcal{I})$ by the arguments above and Corollary 5.10. By Lemma 4.10, $\left.\left(\Upsilon^{0}+\chi\right)\right|_{z_{0}=0}=\chi$. Moreover,

$$
\left.\left(\left(\Upsilon^{0}+\chi\right) \wedge\left(\Upsilon^{0}+\chi\right)\right)\right|_{z_{0}=0}=\chi \wedge \chi
$$

Another application of Lemma 4.10 gives (6.5).

\section{Lemma 6.7.}

$$
d\left(\Theta_{i}-\operatorname{Ad}\left(g_{i}\right)(\chi)\right) \in \Omega_{\widehat{\mathcal{M}}}^{2}\left(\mathfrak{u}_{i}\right)+\mathcal{I} .
$$

Proof. Throughout, we will write $\Upsilon=\Upsilon^{0}+\chi$. As in the beginning of Section 5.2 , we write $\hat{p}_{i}=\hat{g}_{i} g_{i}^{-1}$. By Proposition [5.9, we see that

$$
\operatorname{Ad}\left(g_{i}\right)(\Upsilon) \in \operatorname{Ad}\left(\hat{p}_{i}^{-1}\right)\left(A_{\widetilde{\mathcal{M}}, i}^{\prime}\right)+\hat{p}_{i}^{-1} d \hat{p}_{i}+\left(d g_{i}\right) g_{i}^{-1}+\mathcal{I}
$$

A sequence of calculations using the expression above and Lemma 6.6 produces

$$
\begin{aligned}
d\left(\operatorname{Ad}\left(g_{i}\right) \Upsilon\right) & =\left[\left(d g_{i}\right) g_{i}^{-1}, \operatorname{Ad}\left(g_{i}\right)(\Upsilon)\right]+\operatorname{Ad}\left(g_{i}\right) d(\Upsilon) \\
& \in\left[\left(d g_{i}\right) g_{i}^{-1}, \operatorname{Ad}\left(g_{i}\right)(\Upsilon)\right]-\operatorname{Ad}\left(g_{i}\right)(\Upsilon \wedge \Upsilon)+\mathcal{I} \\
& =\left[\left(d g_{i}\right) g_{i}^{-1}, \operatorname{Ad}\left(\hat{p}_{i}^{-1}\right)\left(A_{\widetilde{\mathcal{M}}_{i},}^{\prime}\right)+\hat{p}_{i}^{-1} d \hat{p}_{i}+\left(d g_{i}\right) g_{i}^{-1}\right] \\
& -\left(\operatorname{Ad}\left(\hat{p}_{i}^{-1}\right)\left(A_{\widetilde{\mathcal{M}}_{i}}^{\prime}\right)+\hat{p}_{i}^{-1} d \hat{p}_{i}+\left(d g_{i}\right) g_{i}^{-1}\right) \wedge\left(\operatorname{Ad}\left(\hat{p}_{i}^{-1}\right)\left(A_{\widetilde{\mathcal{M}}_{i},}^{\prime}\right)+\hat{p}_{i}^{-1} d \hat{p}_{i}+\left(d g_{i}\right) g_{i}^{-1}\right)+\mathcal{I} \\
& \left.=-d g_{i} d g_{i}^{-1}+d \hat{p}_{i}^{-1} d \hat{p}_{i}-\left[\hat{p}_{i}^{-1} d \hat{p}_{i}, \operatorname{Ad}\left(\hat{p}_{i}^{-1}\right) A_{\widetilde{\mathcal{M}}, i}^{\prime}\right)\right]+\mathcal{I} \\
& \left.=-d g_{i} d g_{i}^{-1}+d \hat{p}_{i}^{-1} d \hat{p}_{i}+d\left(\operatorname{Ad}\left(\hat{p}_{i}^{-1}\right) A_{\widetilde{\mathcal{M}}, i}^{\prime}\right)\right)+\mathcal{I} .
\end{aligned}
$$

The last line is equivalent to $d \Phi_{i}-d g_{i} d g_{i}^{-1}+\mathcal{I}\left(\bmod \Omega_{\widehat{\mathcal{M}}^{1}}^{1}\left(\mathfrak{P}_{i}^{1}\right)\right)$. Therefore,

$$
d\left(\Phi_{i}+\left(d g_{i}\right) g_{i}^{-1}-\operatorname{Ad}\left(g_{i}\right)\left(\Upsilon^{0}+\chi\right) \in \Omega_{\widehat{\mathcal{M}}}^{2}\left(\mathfrak{P}_{i}^{1}\right)+\mathcal{I} .\right.
$$

Applying $\bar{\phi}_{i}$ to the equation above gives $d\left(\Theta_{i}-\operatorname{Ad}\left(g_{i}\right)(\chi)\right) \in \Omega_{\widehat{\mathcal{M}}}^{2}\left(\mathfrak{u}_{i}\right)+\mathcal{I}$. 
Proof of Theorem [5.1, part (11). As above, write $\Upsilon=\Upsilon^{0}+\chi$. We have already shown that $d\left(\Theta_{i}-\operatorname{Ad}\left(g_{i}\right)(\chi)\right) \in \Omega_{\widehat{\mathcal{M}}}^{2}\left(\mathfrak{u}_{i}\right)+\mathcal{I}$. A standard, but tedious, calculation shows that

$$
d\left(\Xi_{i}-[\chi, \alpha]\right)=\tau_{\nu}(d \Upsilon+\Upsilon \wedge \Upsilon)-[d \Upsilon+\Upsilon \wedge \Upsilon, \alpha]+\left[\Xi_{i}, \Upsilon\right]+\Omega_{\widehat{\mathcal{M}}}^{1}\left(\mathfrak{g}_{i}^{1} \frac{d z_{i}}{z_{i} \nu}\right),
$$

so by Lemma 6.6, $d\left(\Xi_{i}-[\chi, \alpha]\right) \in \mathcal{I}$.

Finally, by Theorem 3.16, we may identify trivialized framed global deformations $(\mathrm{g}, \bar{V}, \tilde{\nabla})$ on $\mathbb{P}^{1} \times \Delta$ with analytic maps $\sigma: \Delta \rightarrow \widehat{\mathcal{M}}(\mathbf{x}, \mathbf{P}, \mathbf{r})$. We will show that the conditions of Corollary 4.13 are equivalent to the vanishing of $\sigma^{*} \mathcal{I}$. Suppose that $\sigma^{*} \mathcal{I}=0$. By Corollary 5.10, $d \chi+\chi \wedge \chi=0$. It follows that there exists an analytic solution $g \in \mathrm{GL}_{n}(R)$ on $\Delta$ to the integrable differential equation $d g=g \cdot \chi$. In particular, $\chi=g^{-1} d g$.

We observe that $\sigma^{*} \mathcal{I}=\{0\}$ if and only if $\sigma^{*}\left(\Theta_{i}-\operatorname{Ad}\left(g_{i}\right)(\chi)\right)=0$ and $\sigma^{*} \Xi_{i}-$ $[\chi, \alpha]=0$ for all $i$, and the vanishing of these forms is equivalent to conditions (11) and (2) of Corollary 4.13. Also, by Lemma 6.6, $\sigma^{*} \mathcal{I}=\{0\}$ implies $\sigma^{*}(d \Upsilon+\Upsilon \wedge \Upsilon)=$ 0 , which is equivalent to condition (3).

Theorem 5.1 and Lemma 6.6 immediately show that the third conditions in Theorem 4.12 and Corollary 4.13 are redundant.

Theorem 6.8. Let $(\mathrm{g}, \bar{V}, \tilde{\nabla})$ be a good framed deformation. The following statements are equivalent.

(1) $(\mathbf{g}, \bar{V}, \tilde{\nabla})$ is integrable.

(2) $(\mathbf{g}, \bar{V}, \tilde{\nabla})$ is $\mathrm{GL}_{n}(R)$-gauge-equivalent to a deformation satisfying the first two conditions of Theorem 4.12.

(3) There exists $g \in \mathrm{GL}_{n}(R)$ such that the first two conditions of Corollary 4.13 are satisfied.

Proof of Theorem 5.1, part (2). Since $q^{*} \overline{\mathcal{I}}=\mathcal{I}$, and $\mathcal{I}$ is a Pfaffian system by Theorem 5.1 one immediately sees that $\overline{\mathcal{I}}$ is Pfaffian. Specifically, the rank of $\overline{\mathcal{I}}^{1}$ at any point $m \in \widetilde{\mathcal{M}}$ is equal to the rank of $\mathcal{I}^{1}$ at a point in $q^{-1}(m)$.

In order to prove integrability, suppose that $\omega \in \overline{\mathcal{I}}^{1}$. Then, $d\left(q^{*} \omega\right)=q^{*}(d \omega) \in \mathcal{I}$. It now suffices to work on a neighborhood $W \subset \widetilde{\mathcal{M}}$. Choosing a local section $\sigma: W \rightarrow q^{-1}(W)$ of the principal $\mathrm{GL}_{n}(\mathbb{C})$-bundle, we see that $\left.\sigma^{*} \mathcal{I}\right|_{q^{-1}(W)}=\left.\overline{\mathcal{I}}\right|_{W}$. We deduce that $d \omega=\left.\sigma^{*} q^{*}(d \omega) \in \overline{\mathcal{I}}\right|_{W}$.

Finally, suppose that $f: \Delta \rightarrow \widetilde{\mathcal{M}}$. Again, we choose a local section $\sigma: W \rightarrow$ $q^{-1}(W)$ of a neighborhood $W$ containing the image of $\Delta$. By the first part of Theorem [5.1, $f$ corresponds to a framed integrable deformation if and only if $f^{*} \sigma^{*}(\mathcal{I})=0$. However, this is equivalent to the statement that $f^{*}(\overline{\mathcal{I}})=0$ since $\sigma^{*}(\mathcal{I})=\left.\overline{\mathcal{I}}\right|_{W}$.

\section{EXAMPLE}

In this section, we give an explicit example of the system of equations constructed above. We will consider a space of rank $n$ meromorphic connections on $\mathbb{P}^{1}$ with $m$ singularities of slope $\frac{1}{n}$. Let $\mathbf{x}$ be a set of $m$ finite points, and set $P_{i}=I_{i}$ and $r_{i}=1$ for all $i$. If $z$ is the usual coordinate on $\mathbb{P}^{1}$, we write $z_{i}=\left(z-\xi_{i}\right)$. Accordingly, 
$\varpi_{i}=\varpi_{T_{i}}=N+z_{i} E \in \mathfrak{P}_{i}^{1}$, where $N$ is the regular nilpotent matrix in Jordan form and $E$ is the elementary matrix $E_{n 1}$. Note that $\varpi_{i}^{-1}=N^{\prime}+\frac{1}{z_{i}} E^{\prime}$, where $N^{\prime}$ and $E^{\prime}$ are the transposes of $N$ and $E$ respectively.

We choose our one form to be $\nu=d z$, so $\tau_{\nu}=\partial_{z}=\frac{d}{d z}$. Choose a point $\left(U_{i} g_{i}, \alpha_{i}\right)_{i \in I} \in \widehat{\mathcal{M}}$ corresponding to a connection $\nabla$, and write $[\nabla]=\alpha \nu$. Thus, $\alpha \in \alpha_{i \nu}+\Im_{i}^{1}$ at each $i$ and $\sum_{i \in I} \operatorname{Res}_{i}(\alpha \nu)=0$. To simplify calculations, we assume that the normalized formal type of $\nabla$ at $\xi_{i}$ has the representative $\frac{1}{z_{i}}\left(-\frac{a_{i}}{n} \varpi_{i}^{-1}+H_{T}\right)$ under the pairing $\langle,\rangle_{\nu}$.

We write $A_{\widetilde{\mathcal{M}}, i}=\varpi_{i}^{-1} d a_{i}$ and $\operatorname{Ad}\left(g_{i}\right)(\alpha)=\frac{1}{z_{i}}\left(-\frac{a_{i}}{n} \varpi_{i}^{-1}-\frac{1}{n}\left(D_{i}+X_{i}\right)+H_{T}\right)+\mathfrak{g}_{i}$ for some traceless diagonal matrix $D_{i}$ and $X_{i} \in \mathfrak{u}_{i}$. We let $\varrho_{i}$ denote the residue term of $\alpha \nu$ at $x_{i}$. Explicitly, $\varrho_{i}=\phi_{i}\left(z_{i} \alpha\right)=\operatorname{Ad}\left(g^{-1}\right)\left(-\frac{1}{n}\left(N^{\prime}+D_{i}+X_{i}\right)+H_{T}\right)$.

Proposition 7.1. The isomonodromy equations for the system above are

$$
\begin{aligned}
& \left(\left(d g_{i}\right) g_{i}^{-1}, g_{i}\right)=\left(\left[\sum_{j \in I \backslash i} \frac{1}{\xi_{i}-\xi_{j}} \operatorname{Ad}\left(g_{i} g_{j}^{-1}\right)\left(E^{\prime} d a_{j}\right)\right]-\left[N^{\prime} d a_{i}+D_{i} \frac{d a_{i}}{a_{i}}\right]+\mathfrak{u}_{i}, g_{i}\right) \\
& \in\left(\mathfrak{u}_{i} \backslash \mathfrak{g l} \mathfrak{l}_{n}(\mathbb{C})\right) \times_{U_{i}} \mathrm{GL}_{n}(\mathbb{C}) \text { and } \\
& d \varrho_{i}=\sum_{j \in I \backslash\{i\}}\left(\frac{1}{\xi_{j}-\xi_{i}}\left(\left[\operatorname{Ad}\left(g_{j}^{-1}\right) E^{\prime}, \varrho_{i}\right] d a_{j}+\left[\operatorname{Ad}\left(g_{i}^{-1}\right) E^{\prime}, \varrho_{j}\right] d a_{i}\right)\right. \\
& \left.+\frac{1}{n} \frac{1}{\left(\xi_{j}-\xi_{i}\right)^{2}}\left[\operatorname{Ad}\left(g_{i}^{-1}\right) E^{\prime}, \operatorname{Ad}\left(g_{j}^{-1}\right) E^{\prime}\right] d\left(a_{i} a_{j}\right)\right) .
\end{aligned}
$$

Remark 7.2. In this system of partial differential equations, the independent variables are the $a_{i}$ 's, which are essentially the formal types, while the dependent variables are the framings $g_{i}$ and the residues $\varrho_{i}$ of $\alpha \nu$ (which can be written explicitly in terms of $g_{i}, D_{i}$, and $X_{i}$ ).

Proof. First, observe that by definition,

$$
\Phi_{i}=\left(\varpi_{i}^{-1} d a_{i}+D_{i} \frac{d a_{i}}{a_{i}}\right)+\Omega_{\Delta}^{1}\left(\mathfrak{I}_{i}^{1}\right) .
$$

Therefore,

$$
\Upsilon^{0}=\sum_{i \in I} \operatorname{Ad}\left(g_{i}^{-1}\right)\left(z_{i}^{-1} E^{\prime} d a_{i}\right)
$$

and

$$
\phi_{i}\left(\Upsilon^{0}\right)=\sum_{j \in I \backslash\{i\}} \frac{1}{\xi_{i}-\xi_{j}} \operatorname{Ad}\left(g_{j}^{-1}\right)\left(E^{\prime} d a_{j}\right) .
$$

We conclude that equation (1) from Theorem 4.12 is equivalent to (7.1a).

Now, we consider equation (2) of Theorem 4.12. At $\xi_{i}$, applying $\operatorname{Ad}\left(g_{i}\right)$ to the principal part of the curvature gives us

$$
\begin{aligned}
& \quad-z_{i}^{-2} E^{\prime} d a_{i} \in \\
& -\frac{1}{n z_{i}}\left(\varpi_{i}^{-1} d a_{i}+d D_{i}+d X_{i}\right)+\left[\operatorname{Ad}\left(g_{i}\right)\left(\Upsilon^{0}\right)-\left(d g_{i}\right) g_{i}^{-1}, \operatorname{Ad}\left(g_{i}\right)(\alpha)\right]+\Omega_{\Delta}^{1}\left(\mathfrak{g}_{i}\right) .
\end{aligned}
$$


First, we calculate $\left[z_{i}^{-1} E^{\prime}, \operatorname{Ad}\left(g_{i}\right)(\alpha)\right]+\mathfrak{g}_{i}$. Observe that $\left[z_{i}^{-1} E^{\prime}, \operatorname{Ad}\left(g_{i}\right)(\alpha) d z\right]$ is a one form on $\mathbb{P}_{\Delta}^{1}$ with poles along $\mathbf{x}$. Thus,

$$
\left[z_{i}^{-1} E^{\prime}, \operatorname{Ad}\left(g_{i}\right)(\alpha) d z\right] \in\left(-\frac{1}{n z_{i}}\left[z_{i}^{-1} E^{\prime}, N^{\prime} a_{i}+D_{i}+X_{i}-n H_{T}\right]+\frac{1}{z_{i}} Y\right) d z+\mathfrak{g}_{i} d z
$$

where $Y$ is the residue term. By the residue theorem, $Y+\sum_{j \in I} \operatorname{Res}_{j}\left(\left[z_{i}^{-1} E^{\prime}, \operatorname{Ad}\left(g_{i}\right)(\alpha) d z\right]\right)=$ 0 . We conclude that

$$
\begin{aligned}
Y & =\frac{1}{n} \sum_{j \in I \backslash\{i\}} \operatorname{Res}_{j}\left(\left[z_{i}^{-1} E^{\prime}, \operatorname{Ad}\left(g_{i} g_{j}^{-1}\right)\left(\varpi_{i}^{-1} a_{j}+D_{j}+X_{j}-n H_{T}\right)\right] \frac{d z}{z_{j}}\right) \\
& =\frac{1}{n} \sum_{j \in I \backslash\{i\}} \frac{1}{\xi_{j}-\xi_{i}}\left[E^{\prime}, \operatorname{Ad}\left(g_{i} g_{j}^{-1}\right)\left(N^{\prime} a_{j}+D_{j}+X_{j}-n H_{T}\right)\right]-\frac{1}{\left(\xi_{j}-\xi_{i}\right)^{2}}\left[E^{\prime}, \operatorname{Ad}\left(g_{i} g_{j}^{-1}\right) E^{\prime} a_{j}\right] .
\end{aligned}
$$

By Proposition 4.2 we see that

$$
-z_{i}^{-2} E^{\prime} d a_{i}=-\frac{1}{n z_{i}}\left(\varpi_{i}^{-1} d a_{i}\right)+\frac{1}{z_{i}}\left[\varpi_{i}^{-1} d a_{i}, H_{T}\right] .
$$

Comparing (7.2), (7.3), and (7.4), we obtain the condition

$$
\begin{aligned}
& (7.5)-\frac{1}{n z_{i}}\left(d\left(D_{i}+X_{i}\right)+\left[z_{i}^{-1} E^{\prime} d a_{i}, N^{\prime} a_{i}+D_{i}+X_{i}-n H_{T}\right]\right)+ \\
& \frac{1}{z_{i}} Y d a_{i}+\left[\operatorname{Ad}\left(g_{i}\right)\left(\Upsilon^{0}\right)-z_{i}^{-1} E^{\prime} d a_{i}-\left(d g_{i}\right) g_{i}^{-1}, \operatorname{Ad}\left(g_{i}\right)(\alpha)\right]-\frac{1}{z_{i}}\left[\varpi_{i}^{-1} d a_{i}, H_{T}\right] \in \Omega_{\Delta}^{1}\left(\mathfrak{g}_{i}\right) .
\end{aligned}
$$

Now, by 7.1a),

$$
\left[\operatorname{Ad}\left(g_{i}\right) \Upsilon^{0}-z_{i}^{-1} E^{\prime} d a_{i}-\left(d g_{i}\right) g_{i}^{-1}, z_{i}^{-1} E^{\prime} a_{i}\right] \in
$$$$
\left[\phi_{i}\left(\operatorname{Ad}\left(g_{i}\right) \Upsilon^{0}\right)-\left(d g_{i}\right) g_{i}^{-1}, z_{i}^{-1} E^{\prime} a_{i}\right]-\sum_{j \in I \backslash\{i\}} \frac{1}{\left(\xi_{j}-\xi_{i}\right)^{2}}\left[\operatorname{Ad}\left(g_{i} g_{j}^{-1}\right)\left(E^{\prime}\right), E^{\prime}\right] a_{i} d a_{j}+\Omega_{\Delta}^{1}\left(\mathfrak{g}_{i}^{1}\right)
$$$$
=\left[N^{\prime} d a_{i}+D_{i} \frac{d a_{i}}{a_{i}}, z_{i}^{-1} E^{\prime} a_{i}\right]-\sum_{j \in I \backslash\{i\}} \frac{1}{\left(\xi_{j}-\xi_{i}\right)^{2}}\left[\operatorname{Ad}\left(g_{i} g_{j}^{-1}\right)\left(E^{\prime}\right), E^{\prime}\right] a_{i} d a_{j}+\Omega_{\Delta}^{1}\left(\mathfrak{g}_{i}^{1}\right) .
$$

On the other hand,

$$
\begin{aligned}
& {\left[\operatorname{Ad}\left(g_{i}\right) \Upsilon^{0}-z_{i}^{-1} E^{\prime} d a_{i}, N^{\prime} a_{i}+D_{i}+X_{i}-n H_{T}\right] \in} \\
& \quad\left[\phi_{i}\left(\operatorname{Ad}\left(g_{i}\right) \Upsilon^{0}\right), N^{\prime} a_{i}+D_{i}+X_{i}-n H_{T}\right]+\Omega_{\Delta}^{1}\left(\mathfrak{g}_{i}^{1}\right) \\
& \quad=\left[\sum_{j \in I \backslash\{i\}} \frac{1}{\xi_{i}-\xi_{j}} \operatorname{Ad}\left(g_{i} g_{j}^{-1}\right)\left(E^{\prime} d a_{j}\right), N^{\prime} a_{i}+D_{i}+X_{i}-n H_{T}\right]+\Omega_{\Delta}^{1}\left(\mathfrak{g}_{i}^{1}\right) .
\end{aligned}
$$

Finally,

$$
\left[z_{i}^{-1} E^{\prime} d a_{i}, H_{T}\right]-\left[\varpi_{i}^{-1} d a_{i}, H_{T}\right]=\left[-N^{\prime} d a_{i}, H_{T}\right]
$$


We substitute (7.6), (7.7), and (7.8) into (7.5):

$$
\begin{gathered}
d\left(D_{i}+X_{i}\right)-\left[\left(d g_{i}\right) g_{i}^{-1}, N^{\prime} a_{i}+D_{i}+X_{i}-n H_{T}\right] \equiv\left[-N^{\prime} d a_{i}, n H_{T}\right]+ \\
\sum_{j \in I \backslash\{i\}} \frac{1}{\xi_{j}-\xi_{i}}\left[E^{\prime} d a_{i}, \operatorname{Ad}\left(g_{i} g_{j}^{-1}\right)\left(N^{\prime} a_{j}+D_{j}+X_{j}-n H_{T}\right)\right]-\frac{1}{\left(\xi_{j}-\xi_{i}\right)^{2}}\left[E^{\prime}, \operatorname{Ad}\left(g_{i} g_{j}^{-1}\right) E^{\prime}\right] a_{j} d a_{i} \\
+\sum_{j \in I \backslash\{i\}} \frac{1}{\left(\xi_{j}-\xi_{i}\right)^{2}}\left[\operatorname{Ad}\left(g_{i} g_{j}^{-1}\right)\left(E^{\prime}\right), E^{\prime}\right] a_{i} d a_{j} \\
-\sum_{j \in I \backslash\{i\}} \frac{1}{\xi_{i}-\xi_{j}}\left[\operatorname{Ad}\left(g_{i} g_{j}^{-1}\right)\left(E^{\prime} d a_{j}\right), N^{\prime} a_{i}+D_{i}+X_{i}-n H_{T}\right] \quad\left(\bmod \Omega_{\Delta}^{1}\left(\mathfrak{g}_{i}^{1}\right)\right) .
\end{gathered}
$$

Since $\left[N^{\prime} d a_{i}, H_{T}\right]=\frac{1}{n} N^{\prime} d a_{i}$, applying $\operatorname{Ad}\left(g_{i}^{-1}\right)$ and dividing both sides by $-n$ shows that this equation is equivalent to (7.1b).

\section{REFERENCES}

[1] P. Boalch, "Symplectic manifolds and isomonodromic deformations," Adv. Math. 163 (2001), 137-205.

[2] C. Bremer and D. S.Sage, "Moduli spaces of irregular singular connections," arXiv:1004.4411 v1 [math.AG], 2010.

[3] C. J. Bushnell, "Hereditary orders, Gauss sums, and supercuspidal representations of $\mathrm{GL}_{N}$," J. Reine Angew. Math. 375/376 (1987), 184-210.

[4] N. Chriss and V. Ginzburg, Representation theory and complex geometry, Birkhäuser, Boston, 1997.

[5] E. Frenkel, Langlands correspondence for loop groups, Cambridge University Press, New York, 2007.

[6] V. Guillemin and S. Sternberg, Geometric Asymptotics, Mathematical Surveys, No. 14, American Mathematical Society, Providence, 1977.

[7] G. Hector and U. Hirsch, Introduction to the Geometry of Foliations, Part A, Aspects of Mathematics, Friedr. Vieweg and Sohn, Braunschweig, 1981.

[8] M. Jimbo and T. Miwa, "Monodromy preserving deformations of linear differential equations with rational coefficients II," Physica D 2 (1981), 407-448.

[9] M. Jimbo, T. Miwa, and K. Ueno, "Monodromy preserving deformations of linear ordinary differential equations with rational coefficients I," Physica D 2 (1981), 306-352.

[10] B. Malgrange, Équations Différentielles à Coefficients Polynomiaux, Progress in Mathematics, Vol. 96, Birkhäuser Boston, Inc., Boston, MA, 1991.

[11] J. Marsden and T. Ratiu, "Reduction of Poisson Manifolds," Letters in Mathematical Physics, 11 (1986), 161-169.

[12] A. Moy and G. Prasad, "Unrefined minimal $K$-types for $p$-adic groups," Invent. Math. 116 (1994), 393-408.

[13] C. Sabbah, Isomonodromic Deformations and Frobenius Manifolds, Universitext, Springer, Berlin, 2007.

[14] D. S. Sage, "The geometry of fixed point varieties on affine flag manifolds," Trans. Amer. Math. Soc. 352 (2000), 2087-2119.

[15] L. Schlesinger, "Über eine Klasse von Differentialsystemen beliebiger Ordnung mit festen kritischen Punkten," J. Reine Angew. Math., 141 (1912), 96-145.

[16] E. Witten, "Gauge theory and wild ramification," Anal. Appl. (Singap.) 6 (2008), 429-501.

Department of Mathematics, Louisiana State University, Baton Rouge, LA 70803

E-mail address: cbremer@math.1su.edu

E-mail address: sage@math.1su.edu 